\title{
PILARES ESBELTOS DE CONCRETO ARMADO COM SEÇÃO VARIÁVEL
}

JOICE MALAKOSKI

Dissertação apresentada à Escola de Engenharia de São Carlos, da Universidade de São Paulo, como parte dos requisitos para a obtenção do Título de Mestre em Engenharia de Estruturas.

ORIENTADOR: Prof. Dr. Libânio Miranda Pinheiro 
Ficha catalográfica preparada pela Seção de Tratamento da Informação do Serviço de Biblioteca - EESC-USP

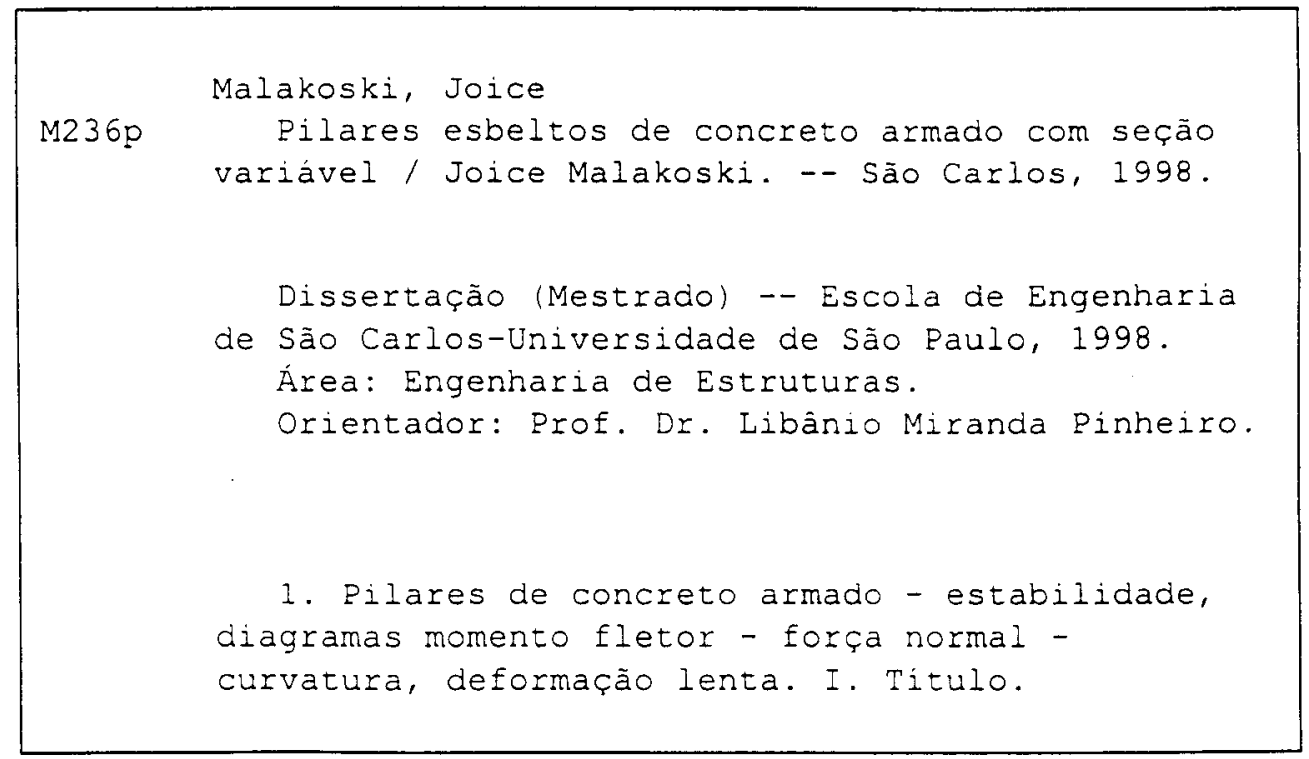


Aos acadêmicos de Engenharia Civil da Universidade

Federal do Paraná.

A Nael, meu irmão e primeiro professor. 


\section{AGRADECIMENTOS}

Ao Professor Libânio Miranda Pinheiro pela orientação, pelo auxílio, pelos ensinamentos, pela compreensão e apoio fomecidos ao longo da elaboração desta dissertação.

À Bibliotecária Maria Nadir Minatel e aos Desenhistas do Departamento de Estruturas da EESC/USP, pela colaboração e eficiência.

A todos os colegas, professores e funcionários do Departamento de Estruturas da EESC/USP pelo companheirismo e atenção.

À Coordenadoria de Aperfeiçoamento de Pessoal de Nivel Superior - CAPES, pela bolsa de estudo concedida.

À Ilka e Sheyla, pela convivência diária, à Regina, pela descontração; as três pela amizade, pelos incentivos e pelo enriquecimento pessoal gerado na busca de soluções para os problemas cotidianos. 


\section{SUMÁRIO}

LISTA DE FIGURAS vii

LISTA DE TABELAS Xi

LISTA DE ABREVIATURAS, SIGLAS E SÍMBOLOS xii

RESUMO XV X

$\begin{array}{lll}\text { ABSTRACT } & \text { xvi }\end{array}$

1 INTRODUÇÃO 1

2 OBJETIVOS 3

3 CONCEITOS DE RESISTÊNCIA DOS MATERIAIS 4

3.1 HIPÓTESES DE CÁLCULO 4

3.2 SOLICITAÇÕES NORMAIS 4

3.3 FLEXÃO 5

3.3.1 Flexão Normal Simples 5

3.3.2 Flexão Obliqua Simples 9

3.3.3 Flexão Normal Composta 12

3.3.4 Flexão Obliqua Composta 14

$\begin{array}{lll}3.4 & \text { EXCENTRICIDADE DA FORÇA NORMAL } & 15\end{array}$

3.5 CONCEITOS DE INSTABILIDADE ELÁSTICA 16

3.5.1 Barras Prismáticas 16

$\begin{array}{lll}3.5 .2 & \text { Barras Não Prismáticas } & 18\end{array}$

4 ESTUDO DAS DEFORMAÇÕES 19

$\begin{array}{lll}4.1 & \text { DEFORMAÇÃO POR FLEXÃO } & 19\end{array}$

$\begin{array}{lll}4.2 & \text { ANALOGIA DE MOHR } & 22\end{array}$ 
4.3 DEFORMAÇÕES NO CONCRETO 24

4.4 DEFORMAÇÕES NO AÇO 30

4.5 ESTADO LIMITE ÚLTIMO

4.5.1 Domínio 1 33

4.5.2 Domínio 2

4.5.3 Dominio $3 \quad 34$

4.5.4 Dominio 4 34

4.5.5 Domínio $4 a \quad 35$

4.5.6 Domínio 5

$5 \quad$ INSTABILIDADE DE BARRAS

5.1 CONCEITOS SOBRE AS CONFIGURAÇÕES DE EQUILÍBRIO 37

5.2 CÁlCULO DA FORÇA CRÍTICA: MÉTODO GERAL 43

5.2.1 Processo do Carregamento Progressivo Proporcional 43

5.2.2 Processo das Excentricidades Progressivas 45

5.3 CÁlCULO DA FORÇA CRÍTICA: MÉTODO DO EQUILÍBRIO 47

6 DIAGRAMAS MOMENTO FLETOR - FORÇA NORMAL CURVATURA

6.1 CURVATURA E DEFORMAÇÃO DAS SEÇÕES 49

6.2 EQUAÇÕES DE EQUILÍBRIO

6.2.1 Seções Retangulares Cheias 58

6.2.2 Seções Retangulares Vazadas 59

6.2.3 Seções Circulares Cheias 60

6.2.4 Seções Circulares Vazadas 61

6.3 EQUAÇÕES ADIMENSIONAIS DO EQUILÍBRIO 62

6.3.1 Seções Retangulares Cheias 64

6.3.2 Seções Retangulares Vazadas 73

6.3.3 Seções Circulares Cheias 78

6.3.4 Seções Circulares Vazadas 103

6.4 ROTEIRO PARA A ELABORAÇÃO DOS DIAGRAMAS 108

7 MÉTODOS PARA OBTENÇÃO DOS MOMENTOS DE SEGUNDA $\begin{array}{ll}\text { ORDEM } & 109\end{array}$ 
7.1 INTEGRAÇÃO NUMÉRICA 109

7.1.1 Regra dos Trapézios 110

7.1.2 Integração do Polinômio Interpolador de Lagrange 112

7.2 ANALOGIA DE MOHR 113

7.2.1 Determinação dos Pesos Elásticos 114

7.2.2 Reações e Esforços na Barra Equivalente 115

7.2.3 Número de Iterações 117

$\begin{array}{lll}7.3 \text { EXEMPLOS } & 117\end{array}$

8 VERIFICAÇÃO DA SEGURANÇA 129

8.1 ESTADOS LIMITES 129

8.1.1 Estados Limites Últimos 129

8.1.2 Estados Limites de Utilização 130

8.2 AÇÕES 130

8.2.1 Combinações Últimas Segundo A NBR-8641/1984 131

8.2.2 Combinações Últimas Segundo o CEB-1990 132

8.2.3 Combinações de Utilização 134

8.3 RESISTÊNCIA DE CÁLCULO 135

8.3.1 Valores da NBR-6118/1978 135

8.3.2 CEB-1990 136

8.4 FLEXÃO OBLÍQUA 136

8.4.1 NBR-6118/1978 137

8.4.2 CEB-1990 137

8.5 DEFORMAÇÕES 138

$\begin{array}{lll}8.5 .1 & N B R-6118 / 1978 & 138\end{array}$

8.5.2 CEB-1990 139

8.6 IMPERFEIÇÕES GEOMÉTRICAS 141

8.7 FLUENNCIA 142

8.7.1 NBR-6118/1978 143

8.7.2 CEB-1990 145

9 ROTINA COMPUTACIONAL 147

9.1 EXEMPLO DE APLICAÇ̃̃O 147

$\begin{array}{lll}9.2 & \text { PROGRAMA } & 149\end{array}$ 
$\begin{array}{lll}10 & \text { CONCLUSÃO } & 168\end{array}$

$\begin{array}{ll}\text { ANEXO } & 169\end{array}$

REFERÊNCIAS BIBLIOGRÁFICAS 174 


\section{LISTA DE FIGURAS}

Figura 3.1:- Esforços solicitantes na flexão 5

Figura 3.2:- Esforços solicitantes na flexão normal simples 6

Figura 3.3:- Distribuição das tensões na flexão normal simples 6

$\begin{array}{ll}\text { Figura 3.4:- Momento fletor na flexão oblíqua simples } & 10\end{array}$

$\begin{array}{ll}\text { Figura 3.5:- Distância de um ponto à linha neutra } & 11\end{array}$

Figura 3.6:- Esforços solicitantes na flexão normal composta 13

Figura 3.7:- Posição da linha neutra na flexão composta obliqua $\quad 14$

$\begin{array}{ll}\text { Figura 3.8:- Barra prismática sujeita à força axial } & 16\end{array}$

$\begin{array}{ll}\text { Figura 3.9:- Comprimento de flambagem } & 17\end{array}$

$\begin{array}{ll}\text { Figura 4.1:- Linha elástica } & 20\end{array}$

$\begin{array}{ll}\text { Figura 4.2:- Curvatura proveniente da flexão } & 20\end{array}$

$\begin{array}{ll}\text { Figura 4.3:- Apoio simples } & 24\end{array}$

Figura 4.4:- Diagrama simplificado $(\sigma-\varepsilon)$ para o concreto 25

Figura 4.5:- Diagrama $(\sigma-\varepsilon)$ para o concreto - CEB-1990 26

Figura 4.6:- Possiveis diagramas $(\sigma-\varepsilon)$ para cálculo dos deslocamento 30

Figura 4.7:- Diagrama $(\sigma-\varepsilon)$ de cálculo para aços classe A 31

Figura 4.8:- Diagrama $(\sigma-\varepsilon)$ de cálculo para aços classe A 32

Figura 4.9:- Deformações no dominio 1

Figura 4.10:- Deformações no domínio 2

Figura 4.11:- Deformações no domínio 3

Figura 4.12:- Deformações no dominio 4 
Figura 4.13:- Deformações no domínio 4a 35

Figura 4.14:- Deformações no dominio 5

Figura 5.1:- Barra com força axial centrada 38

$\begin{array}{ll}\text { Figura 5.2:- Diagrama }(\sigma-\varepsilon) & 38\end{array}$

Figura 5.3:- Curvas força-deformação 39

Figura 5.4:- Barra com força axial excêntrica 39

Figura 5.5:- Deformações e tensões em uma seção, material elástico não linear $\quad 40$

Figura 5.6:- Instabilidade na flexo-compressão, material elástico não linear 42

Figura 5.7:- Gráfico carregamento proporcional-deslocamento 43

Figura 5.8:- Etapas de Carregamento 44

Figura 5.9:- Diagrama força-deslocamento 45

Figura 5.10:- Etapas de cálculo:- Processo das excentricidades progressivas $\quad 46$

Figura 5.11:- Determinação do valor de $e_{1, \text { crit }}$

Figura 5.12:- Determinação do valor de $\mathrm{M}_{1, \text { crit }}$

Figura 5.13:- Etapas do processo do deslocamento de referência 48

Figura 5.14:- Deslocamentos calculados para $\mathrm{N}_{\mathrm{d}} \quad 48$

Figura 6.1:- Deformações em uma seção de concreto armado 50

Figura $6.2:-$ Deformações máximas convencionais para uma seção
de concreto armado

Figura 6.3:- Convenção para variação do parâmetro adimensional $\beta_{\mathrm{x}}$

Figura 6.4:- Deformação em uma seção de concreto inteiramente tracionada 54

Figura 6.5:- Deformações em uma seção de concreto parcialmente comprimida 55

Figura 6.6:- Deformações em uma seção de concreto inteiramente comprimida 56

Figura 6.7:- Deformações em uma seção retangular de concreto armado 59

Figura 6.8:- Deformações um uma seção retangular vazada de concreto armado 60 
Figura 6.9:- Deformações um uma seção circular de concreto armado 61

Figura 6.10:- Deformações um uma seção circular vazada de concreto armado $\quad 62$

Figura 6.11:- Tensões em uma seção de concreto armado, diagrama parábolaRetângulo

Figura 6.12:- Tensões em uma seção de concreto armado, diagrama CEB-1990

Figura 6.13:- Trechos para a localização dos pontos $\mathrm{y}_{2}$ e $\mathrm{y}_{3}$

Figura 6.14:- Esquema de cálculo das expressões adimensionais para uma seção vazada

Figura 6.15:- Possiveis localizações de $y_{2}$ 75

Figura 6.16:- Possiveis localizações de $y_{3}$ 76

Figura 6.17:- Possiveis localizações de $y_{3}$ (negativo) 76

Figura 6.18:- Seção circular de raio unitário 77

Figura 7.1:- Diagrama de momentos fletores 110

Figura 7.2:- Diagrama de uma função

Figura 7.3:- Diagramas de curvaturas e deslocamentos

Figura 7.4:- Diagrama de uma função

Figura 7.5:- Carregamento da barra equivalente 115

Figura 7.6:- Barras equivalentes 116

Figura 7.7:- Linha elástica de um pilar engastado na base

Figura 7.8:- Pilar bi-rotulado

Figura 7.9:- Diagrama momento fletor - força normal - curvatura para uma seção retangular simétrica

Figura 8.1:- Substituição da flexo-compressão obliqua

Figura 8.2:- Condições para a decomposição da força obliqua

Figura 8.3:- Deformações no concreto sob tensão constante 
Figura 8.4:- Influência da intensidade e duração do carregamento nas deformações do concreto

Figura 8.5:- Diagrama $\sigma-\varepsilon$ do concreto sob o efeito da fluência

Figura 9.1:- Pilar livre-engastado com seção transversal variável

Figura A. 1:- Forma das barras abrangida pela tabela A.1 170

Figura A.2:- Casos de vinculação correspondentes à tabela A.2 


\section{LISTA DE TABELAS}

Tabela 3.1:- Transformação de vínculos para barra conjugada

Tabela 7.1:- Valores das curvaturas exatas

Tabela 7.2:- Deslocamentos transversais

Tabela 7.3:- Momentos fletores e curvaturas

Tabela 8.1:- Fatores parciais $\gamma_{\mathrm{f}}$ - valores básicos

133

Tabela 8.2:- Fatores de combinação $\Psi$

Tabela 8.3:- Coeficiente de minoração - CEB-1990

Tabela 9.1:- Valores dos deslocamentos e momentos finais

Tabela A.1:- Valores de $I_{\mathbf{f}} / I_{b}$ 


\section{LISTA DE ABREVIATURAS, SIGLAS E SÍMBOLOS}

$\alpha \quad$ - fração da força normal que produz fluência

$A_{c} \quad$ - área de concreto de uma seção transversal

$A_{d} \quad$ - ação acidental

As - área de aço de uma seção transversal

CEB - Comité Euro-Internacional du Béton

d $\quad$ - altura útil

$\varepsilon_{\mathrm{c}} \quad$ - deformação específica à compressão do concreto

$\varepsilon_{\mathrm{c} 2} \quad$ - deformação específica do concreto na borda mais comprimida

$\varepsilon_{3 / 7} \quad$ - deformação específica no concreto distante (3/7)h da borda mais comprimida

$\varepsilon_{\mathrm{cd}} \quad$ - deformação específica do concreto

$\varepsilon_{\mathrm{s}} \quad$ - deformação específica do aço da armadura tracionada

$e_{c} \quad$ - excentricidade adicional remanescente da fluência

$e_{o} \quad$ - excentricidade acidental

$e_{a} \quad$ - excentricidade acidental decorrente de imperfeições geométricas

$\mathrm{e}_{1 . \mathrm{crit}} \quad$ - excentricidade crítica

$\mathrm{e}_{\mathrm{lg}}$ - excentricidade de $1^{\mathrm{a}}$ ordem, incluindo excentricidade acidental

$E_{c} \quad$ - módulo de elasticidade do concreto

$\mathrm{E}_{\mathrm{cl}} \quad$ - módulo de elasticidade secante do concreto

$\mathrm{E}_{\mathrm{cs}} \quad$ - módulo de elasticidade reduzido do concreto 
$E_{\mathrm{s}} \quad$ - módulo de elasticidade do aço

$\varphi \quad$ - deslocamento angular

$\phi \quad$ - coeficiente de fluência

$\phi_{(\text {to.tx) }}$ - função de fluência real do problema

$\mathrm{f}_{\mathrm{cd}} \quad$ - resistência à compressão de cálculo do concreto

$\mathrm{f}_{\mathrm{ck}}$ - resistência à compressão característica do concreto

$\mathrm{f}_{\mathrm{yk}}$. resistência à compressão de cálculo do aço

$f_{\text {yd }} \quad$ - resistência à compressão característica do aço

$\mathrm{f}_{\mathrm{cm}} \quad$ - resistência à compressão média do concreto

$\gamma_{c} \quad$ - coeficiente de minoração da resistência do concreto

$\gamma_{\mathrm{s}}$ - coeficiente de minoração da resistência do aço

$\eta \quad$ - relação entre a área de uma barra e a ára de aço total da seção

i - raio de giração

Ifl - momento de inércia de flambagem

$\mathrm{I}_{\mathrm{x}} \quad$ - momento de inércia em relação ao eixo $\mathrm{x}$

$I_{y} \quad$ - momento de inércia em relação ao eixo y

$\lambda \quad$ - índice de esbeltez

$l_{\mathrm{fl}} \quad$ - comprimento de flambagem

$\mathrm{I}_{\mathrm{LN}} \quad$ - momento de inércia em relação à linha neutra

LCG - linha que passa pelo centro de gravidade de uma seção

LN - linha neutra

$l_{\mathrm{o}} \quad$ - distância entre seções de momento fletor nulo

$\mu_{\mathrm{I}} \quad$ - momento fletor adimensional 


$$
\begin{array}{ll}
M_{I} \quad \text { - momento fletor resistente } \\
M_{s} \quad \text { - momento estático } \\
M_{x} \quad \text { - componente do momento na direção x } \\
M_{y} \quad \text { - componente do momento na direção y } \\
U_{I} \quad \text { - força normal adimensional } \\
N_{g} \quad \text { - carga de longa duração que produz fluência } \\
N_{l} \quad \text { - força normal resistente } \\
\text { NBR } \quad \text { - Norma Brasileira }
\end{array}
$$$$
\Psi \quad \text { - fator de combinação }
$$$$
1 / \mathrm{r} \quad \text { - curvatura }
$$$$
\mathrm{R}_{\mathrm{c}} \quad \text { - resultante das tensões de compressão }
$$$$
R_{t} \quad \text { - resultante das tensões de tração }
$$$$
\sigma \quad \text { - tensão normal }
$$$$
\sigma_{c} \quad \text { - tensão normal no concreto }
$$$$
\sigma_{\mathrm{s}} \quad \text { - tensão normal no aço }
$$$$
\text { V - força cortante }
$$$$
\boldsymbol{V} \text { - deslocamento transversal }
$$

$\omega \quad$ - taxa de armadura

W - módulo de resistência ou fator de forma

x - distância da borda mais comprimida à linha neutra

yo - distância da linha neutra ao eixo do centro de gravidade da seção

$Y_{s} \quad$ - distância das barras de aço ao eixo do centro de gravidade

Z - braço de alavanca de um binário 


\section{RESUMO}

MALAKOSKI, J. (1998) Pilares esbeltos de concreto armado com seção variável, 175p. Dissertação (mestrado) - Escola de Engenharia de São Carlos. Universidade de São Paulo

São descritas as recomendações da Norma Brasileira NBR-6118/1978 e do Código Modelo do Comité Euro-internacional du Béton CEB-1990 para a verificação da estabilidade de pilares esbeltos de concreto armado com seção variável submetidos à flexão normal composta, empregando-se a teoria do método geral. Para a obtenção dos momentos de segunda ordem são descritos os métodos de Engesser-Vianello e da integração numérica das curvaturas das seções transversais ao longo do pilar. Para a determinação do momento fletor absorvido pelas seções em função da curvatura proveniente da flexão do pilar sob a ação de uma força normal, desenvolvem-se as expressões para seções retangulares e circulares (cheias e vazadas) com base nas relações de tensão-deformação dos materiais segundo a norma e o código supracitados. Apresenta-se também um programa para microcomputador, elaborado em linguagem PASCAL, destinado à verificação da estabilidade de pilares com opção de adoção dos critérios da NBR-6118/1978 ou do CEB-1190. Não foram abordados os efeitos decorrentes de vibrações, fazendo-se apenas a descrição dos métodos de consideração dos efeitos decorrentes da deformação lenta.

Palavras-chave: Pilares de concreto armado - estabilidade, diagramas momento fletor - força normal - curvatura, deformação lenta 


\begin{abstract}
MALAKOSKI, J. (1998) Concrete slender columns with variable cross sections, 175p. Dissertação (mestrado) - Escola de Engenharia de São Carlos. Universidade de São Paulo

The recommendations of Brazilian Code NBR-6118/1978 and Comité EuroInternacional du Béton Model Code CEB-1990 are described, for stability verification of concrete slender columns with variable cross sections, subjected to axial load and bending moment, using the exact method. To obtain the second order bending moments, the Engesser-Vianello method and the numeric integration of the cross section curvatures along the longitudinal axial of column method are described. To obtain of bending moment supported by the cross sections due to curvature resultant of the column flexure under axial load, expressions for rectangular and circular (full and hollow) cross sections are developed, using stress-strain relations for materials proposed by model codes above mentioned. It's also presented a software written in PASCAL language for microcomputer and destined to column stability verification, with option to adopt the NBR-6118/1978 or CEB-1990 model code recommendations. Effects due to vibrations were nor included. Methods to take in account creep effects were described.
\end{abstract}

Keywords: Reinforced concrete columns - stability, second order effects, bending moment - axial force - curvature diagrams, creep 


\section{INTRODUÇÃO}

Entendem-se por pilares esbeltos aqueles em que os efeitos de segunda ordem devem ser considerados. A Associação Brasileira de Normas Técnicas, na NBR-6118/1978 (Procedimento para Projeto e Execução de Obras de Concreto Armado), e o Código Modelo/1990 do Comité Euro-Internacional du Béton (CEB) apresentam limites de esbeltez acima dos quais, além dos esforços de primeira ordem, que abrangem os esforços iniciais devidos às cargas aplicadas à estrutura e os esforços devidos às excentricidades acidentais, também devem ser considerados os momentos fletores decorrentes das deformações da própria estrutura.

$\mathrm{Na}$ análise de pilares isolados, que tanto podem ser elementos únicos sujeitos à compressão ou partes integrantes de uma estrutura na qual podem ser considerados isolados para efeito de projeto, há ainda que se levar em conta a não linearidade física dos materiais empregados.

Os efeitos decorrentes de vibrações não foram incluídos neste trabalho, constando apenas a descrição das formas de consideração dos efeitos oriundos da deformação lenta. Também não foram objeto de estudo métodos simplificados (pilar padrão, pilar padrão melhorado ou momento complementar - NBR-6118/1978, item 4.1.1.3.C), por serem recomendados para barras retas de seção e armadura constantes ao longo do comprimento da peça.

No capítulo 2 são apresentados os objetivos deste trabalho que, de uma forma didática, procura fornecer informações relativas ao dimensionamento e verificação da estabilidade de pilares esbeltos de concreto armado com seção variável.

Definições e conceitos básicos da resistência dos materiais, que auxiliam na compreensão do comportamento das estruturas de concreto submetidas a solicitações normais, encontram-se reunidos no capítulo 3.

O capítulo 4 trata das deformações transversais dos elementos sujeitos à flexão, tratando também das deformações longitudinais ocorridas no aço e no 
concreto, apresentando-se os diagramas tensão-deformação propostos pela NBR6118/1978 e pelo CEB-1990.

No capítulo 5 foram abordados conceitos básicos sobre o comportamento de barras sujeitas à compressão e à flexo-compressão, além de métodos para a obtenção de cargas críticas.

As expressões destinadas à obtenção do momento fletor absorvido por seções retangulares e circulares de concreto armado (cheias e vazadas), em função da curvatura proveniente da flexão da barra sob a ação de uma força normal, são apresentadas no capítulo 6 .

No capítulo 7 encontra-se a descrição dos métodos de obtenção dos momentos fletores de segunda ordem, descrevendo-se a integração numéricas das curvaturas e a analogia de Mohr.

As ações e efeitos que devem ser levados em consideração para a verificação da segurança contra os possíveis estados limites são listados no capítulo 8.

A rotina computacional desenvolvida para a verificação de pilares de concreto com seções retangulares e circulares (cheias e vazadas) é apresentada no capítulo 9, juntamente com um exemplo de cálculo para seção retangular cheia, adotando-se as recomendações da NBR-6118/1978 e do CEB-1990. 


\section{OBJETIVOS}

O objetivo deste trabalho é didático, visando fornecer informações relativas ao dimensionamento e à verificação da estabilidade de pilares esbeltos de concreto com seção variável pelo método geral, partindo de conceitos básicos da resistência dos materiais, desenvolvendo as expressões utilizadas na elaboração dos ábacos de dimensionamento e chegando a elaboração de rotina computacional para a verificação e dimensionamento dos pilares, objetivando ainda:

- expor os critérios e as recomendações da NBR-6118/1978 e do CEB/1990 quanto ao dimensionamento e à verificação da estabilidade de pilares isolados de concreto armado com seção variável;

- expor a teoria do método geral e do método do equilíbrio;

- elaborar rotinas informatizadas de cálculo, em linguagem PASCAL, para a verificação de pilares isolados esbeltos de concreto armado com seção variável e para a obtenção de diagramas momento fletor - força normal - curvatura (parte integrante do programa destinado à verificação dos pilares), segundo as diretrizes da NBR-6118/1978 e CEB/1990, para seções transversais retangulares e circulares, cheias e vazadas. 


\section{CONCEITOS DE RESISTÊNCIA DOS MATERIAIS}

Serão apresentados neste capítulo os conceitos básicos da resistência dos materiais, relativos à flexão e à flambagem, que auxiliam na compreensão do comportamento das estruturas de concreto submetidas a solicitação normais.

\subsection{HIPÓTESES DE CÁLCULO}

Na Resistência dos Materiais, para peças submetidas a flexão, é admitida a hipótese de Bernoulli - as seções inicialmente planas permanecem planas após a deformação.

Para que esta hipótese seja válida para o concreto armado no estado limite último, segundo Fusco (1986), deve ser satisfeita a relação.

$$
\left.\frac{l_{0}}{\mathrm{~d}}\right\rangle_{2}
$$

onde $\boldsymbol{l}_{\mathbf{0}}$ é a distância entre seções de momento fletor nulo e $\mathbf{d}$ é a altura útil da seção transversal.

Admite-se que a armadura e o concreto que a envolve sejam perfeitamente solidários, havendo igualdade de deformação específica entre a barra da armadura e o concreto que lhe é adjacente.

\subsection{SOLICITAÇÕES NORMAIS}

As solicitações que produzem tensões normais à seção denominam-se solicitações normais, as quais incluem momentos fletores e forças normais. 
Quando uma seção é solicitada apenas por um momento fletor (flexão pura), os esforços resistentes dão origem a tensões de tração e de compressão.

\subsection{FLEXÃO}

Pode-se classificar a flexão nos elementos estruturais segundo a natureza (flexão simples ou composta) e direção (flexão normal ou oblíqua) dos esforços solicitantes.

Considerando-se os esforços atuantes na seção transversal da barra indicada na fig. 3.1, a existência da força cortante $\mathbf{V}$ não altera a distribuição das tensões normais devidas a $\mathbf{M}$, visto que as tensões decorrentes de $\mathbf{V}$ são tangenciais e estão contidas no plano da seção.

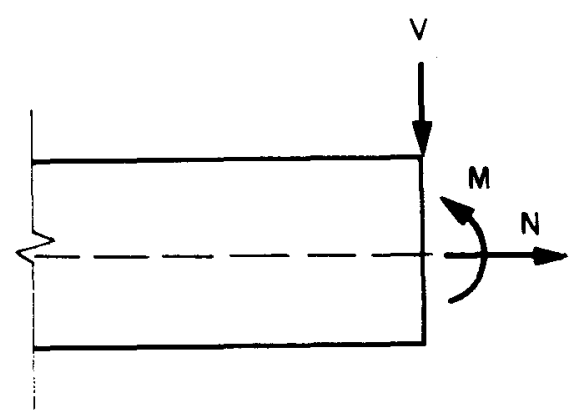

Fig. 3.1 - Esforços solicitantes na flexão.

\subsubsection{FLEXÃO NORMAL SIMPLES}

Têm-se como esforços solicitantes $\mathbf{M}$ e $\mathbf{V}$, sendo que o plano de flexão de $\mathbf{M}$ contém um dos eixos centrais de inércia. $O$ plano de ação do momento fletor é perpendicular ao plano da seção e a força contida no plano da seção passa pelo centro de gravidade desta (fig. 3.2).

As resultantes das tensões de compressão $\left(R_{c}\right)$ e de tração $\left(R_{t}\right)$, originadas pelo momento fletor $(M)$, têm a mesma intensidade, porém seus sentidos são opostos $\left(R_{c}=-R_{t}\right)$. As resultantes das tensões de tração e de compressão formam um binário cujo braço de alavanca é z. Tem-se como condição de equilibrio (convencionando-se positivos os sentidos apresentados na fig. 3.1):

$$
R_{t} \cdot z=-R_{c} \cdot z=M
$$




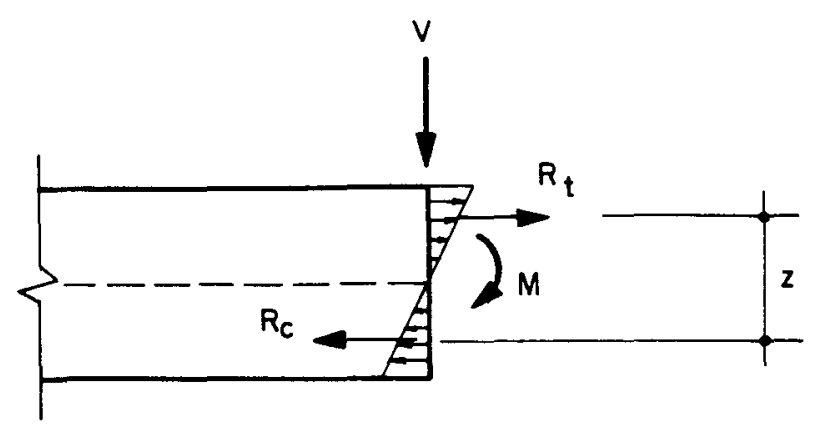

Fig. 3.2. - Esforços solicitantes na flexão normal simples.

Considere-se o plano de ação do momento fletor coincidente com o eixo central de inércia y de uma seção qualquer (fig. 3.3a), para a qual são válidas a lei de Navier e a hipótese de Bernoulli. Estas hipóteses de cálculo podem ser feitas desde que as propriedades físicas (peso específico, densidade) e mecânicas (resistência à tração, resistência à compressão) do material sejam as mesmas de ambos os lados do eixo considerado e que as dimensões da seção transversal não sejam muito elevadas. Desta forma, pode-se dizer que as tensões distribuem-se uniformemente em uma faixa de altura infinitesimal dy, transversal ao plano de ação do momento fletor, e de forma linear ao longo de toda a seção (fig. 3.3b), segundo a direção do eixo considerado.

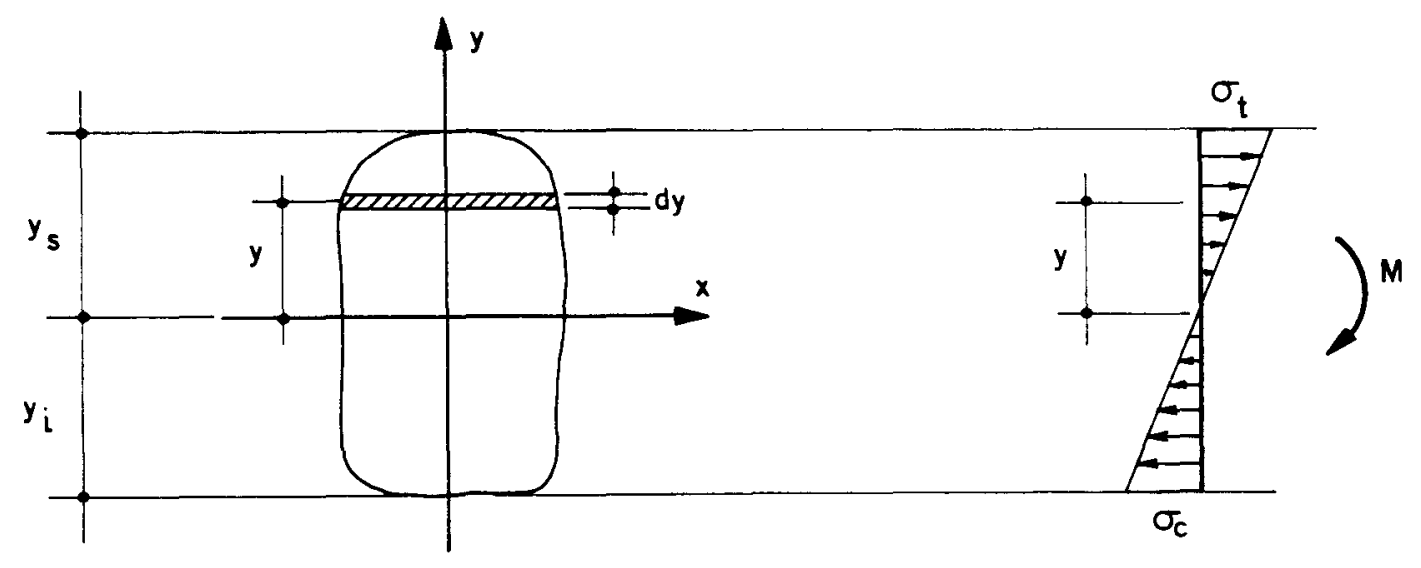

(0)

(b)

Fig. 3.3 - Distribuição das tensões na flexão normal simples. 
Pela lei de Navier, as tensões normais $(\sigma)$ variam linearmente com a distância da faixa considerada ao eixo $\mathbf{x}$, representada na fig. 3.3 por $\mathbf{y}$.

$$
\sigma=\mathrm{a} \cdot \mathrm{y}+\mathrm{c}
$$

Chamando de $\mathbf{R}$ a resultante das tensões originadas pelo momento fletor e de b a largura da faixa considerada, tem-se para toda a seção:

$$
R=R_{t}+R_{c}=\int_{-y_{i}}^{y_{s}} \sigma \cdot b \cdot d y
$$

Substituindo 3.3 em 3.4 :

$$
R=0=a \int_{-y_{i}}^{y_{s}} y \cdot b \cdot d y+c \int_{-y_{i}}^{y_{s}} b \cdot d y
$$

A primeira integral fornece o momento estático de toda a seção, sendo portanto nula; a segunda fornece a área da seção, assim:

$$
\begin{aligned}
& \mathrm{R}=0=\mathrm{a} \cdot 0+\mathrm{c} \cdot \mathrm{A} \\
& \text { Portanto, } \mathrm{c}=0 \mathrm{e} \\
& \sigma=\mathrm{a} \cdot \mathrm{y}
\end{aligned}
$$

Fazendo a somatória dos momentos devidos às forças provenientes das tensões resultantes, tem-se:

$$
\begin{aligned}
& M=\int_{-y_{i}}^{y_{s}} \sigma \cdot y \cdot b \cdot d y=\int_{-y_{i}}^{y_{s}} y \cdot a \cdot y \cdot b \cdot d y \\
& M=a \int_{-y_{i}}^{y_{s}} y^{2} \cdot b \cdot d y
\end{aligned}
$$




$$
\mathrm{M}=\mathrm{a} . \mathrm{I}_{\mathrm{LN}}
$$

onde $\mathbf{I}_{\mathbf{L N}}$ é o momento de inércia da seção em relação à LN (eixo de tensões nulas).

De 3.5:

$$
\begin{aligned}
& \mathrm{M}=\frac{\sigma}{\mathrm{y}} \mathrm{I}_{\mathrm{LN}} \\
& \sigma=\frac{\mathrm{M}}{\mathrm{W}}
\end{aligned}
$$

onde $\mathbf{W}=\mathbf{I}_{\mathbf{L N}} / \mathbf{y}$ é o módulo de resistência ou fator de forma da seção.

Para se determinar o braço de alavanca $(\mathrm{z})$ do binário:

$$
\begin{aligned}
& \mathrm{z}=\left|\frac{\mathrm{M}}{\mathrm{R}_{\mathrm{t}}}\right|=\left|\frac{\mathrm{M}}{-\mathrm{R}_{\mathrm{c}}}\right| \\
& \mathrm{R}_{\mathrm{t}}=\int_{0}^{\mathrm{y}_{\mathrm{s}}} \sigma \cdot \mathrm{b} \cdot \mathrm{dy}=\frac{\mathrm{M}}{\mathrm{I}_{\mathrm{LN}}} \int_{0}^{\mathrm{y}_{\mathrm{s}}} \mathrm{y} \cdot \mathrm{b} \cdot \mathrm{dy} \\
& \mathrm{R}_{\mathrm{t}}=\frac{\mathrm{M}}{\mathrm{I}_{\mathrm{LN}}} \mathrm{M}_{\mathrm{s}}
\end{aligned}
$$

onde $\mathbf{M}_{\mathbf{s}}$ é o momento estático da parte tracionada em relação à LN. Portanto:

$$
z=\frac{I_{L N}}{M_{s}}
$$

O braço de alavanca do binário formado pelos esforços resistentes depende da forma e das dimensões da seção transversal. As seções mais resistentes à flexão 
apresentam maior área de material afastada da linha neutra (maior módulo de resistência).

\subsubsection{FLEXÃO OBLÍQUA SIMPLES}

O plano de ação do momento fletor (M) é perpendicular à seção transversal, porém não coincide com nenhum dos eixos centrais de inércia; a força $\mathbf{V}$ contida no plano da seção passa pelo centro de gravidade. Pode-se decompor o momento fletor em dois outros, segundo os eixos centrais de inércia. Decompondo-se o vetormomento $\mathbf{M}$ nas componentes $\mathbf{M}_{\mathbf{x}}$ e $\mathbf{M}_{\mathbf{y}}$, respectivamente nas direções dos eixos $\mathbf{x}$ e $\mathbf{y}$ (ver fig. 3.4), tem-se:

$$
\begin{aligned}
& M_{x}=M \cdot \cos \left(\alpha_{0}-90^{\circ}\right)=M \cdot \operatorname{sen} \alpha_{0} \\
& M_{y}=M \cdot \operatorname{sen}\left(\alpha_{o}-90^{\circ}\right)=-M \cdot \cos \alpha_{0}
\end{aligned}
$$

Tem-se agora os vetores-momento cujos planos de ação coincidem com os eixos centrais de inércia. As tensões originadas $\left(\sigma_{\mathrm{x}}\right.$ e $\left.\sigma_{\mathrm{y}}\right)$ têm a mesma direção e são somadas algebricamente para a obtenção da tensão resultante nos pontos desejados. Para as tensões de tração e respectiva soma algébrica, tem-se:

$$
\begin{aligned}
& \sigma_{\mathrm{x}}=\frac{\mathrm{M}_{\mathrm{x}}}{\mathrm{I}_{\mathrm{x}}} \mathrm{y}=\frac{\mathrm{M} \cdot \operatorname{sen} \alpha_{\mathrm{o}}}{\mathrm{I}_{\mathrm{x}}} \mathrm{y} \\
& \sigma_{\mathrm{y}}=\frac{\mathrm{M}_{\mathrm{y}}}{\mathrm{I}_{\mathrm{y}}}(-\mathrm{x})=\frac{\mathrm{M} \cdot \cos \alpha_{\mathrm{o}}}{\mathrm{I}_{\mathrm{y}}} \mathrm{x} \\
& \sigma=\frac{\mathrm{M} \cdot \operatorname{sen} \alpha_{\mathrm{o}}}{\mathrm{I}_{\mathrm{x}}} \mathrm{y}+\frac{\mathrm{M} \cdot \cos \alpha_{\mathrm{o}}}{\mathrm{I}_{\mathrm{y}}} \mathrm{x}
\end{aligned}
$$

Para a obtenção da equação da linha neutra $(\sigma=0)$, que é uma reta passando pelo centro de gravidade da seção, faz-se:

$$
0=\frac{M \cdot \operatorname{sen} \alpha_{0}}{I_{x}} y+\frac{M \cdot \cos \alpha_{0}}{I_{y}} x
$$


Desta expressão, resulta:

$$
y=-\frac{I_{x}}{I_{y}} x \cdot \operatorname{cotg} \alpha_{0} \quad\left(\alpha<\alpha_{0}\right)
$$

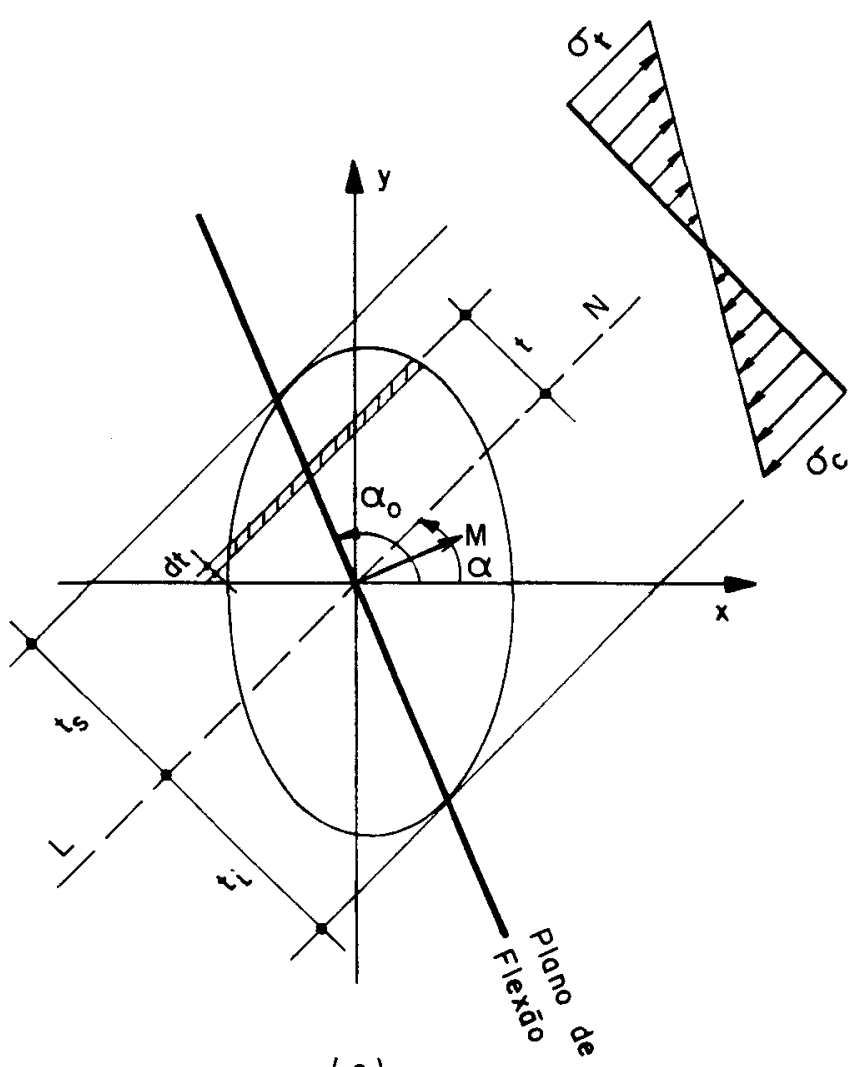

(a)

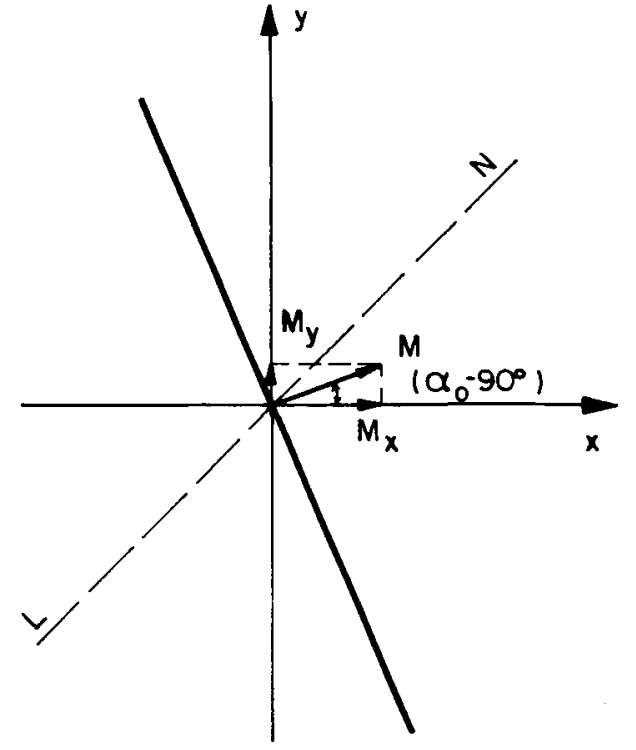

(b)

Fig. 3.4 - Momento fletor na flexão oblíqua simples.

Para a obtenção da inclinação da linha neutra, que forma um ângulo $\alpha$ com o eixo $x$, considerando que $\operatorname{tg} \alpha=\mathbf{y} / \mathbf{x}$, de 3.9 obtém-se:

$$
\operatorname{tg} \alpha=-\frac{I_{x}}{I_{y}} \operatorname{cotg} \alpha_{0}
$$

Em uma faixa paralelá à linha neutra, de altura $\mathrm{dt}$, as tensões são constantes ao longo da largura desta faixa (fig. 3.4a) e proporcionais à distâncias à linha neutra. Com base em 3.8 e 3.10 , obtém-se. 


$$
\begin{aligned}
& \sigma=\frac{M \cdot \operatorname{sen} \alpha_{0}}{I_{x}}\left(y+\frac{I_{x} \cdot \cos \alpha_{0}}{I_{y} \cdot \operatorname{sen} \alpha_{0}} x\right) \\
& \sigma=\frac{M \cdot \operatorname{sen} \alpha_{0}}{I_{x}}(y-x \cdot \operatorname{tg} \alpha)
\end{aligned}
$$

De acordo com a fig. 3.5 , onde $t$ é a distância de um ponto qualquer da seção em relação à linha neutra, tem-se:

$$
\sigma=\frac{M \cdot \operatorname{sen} \alpha_{0}}{I_{x}}\left(\frac{t}{\cos \alpha}\right)
$$

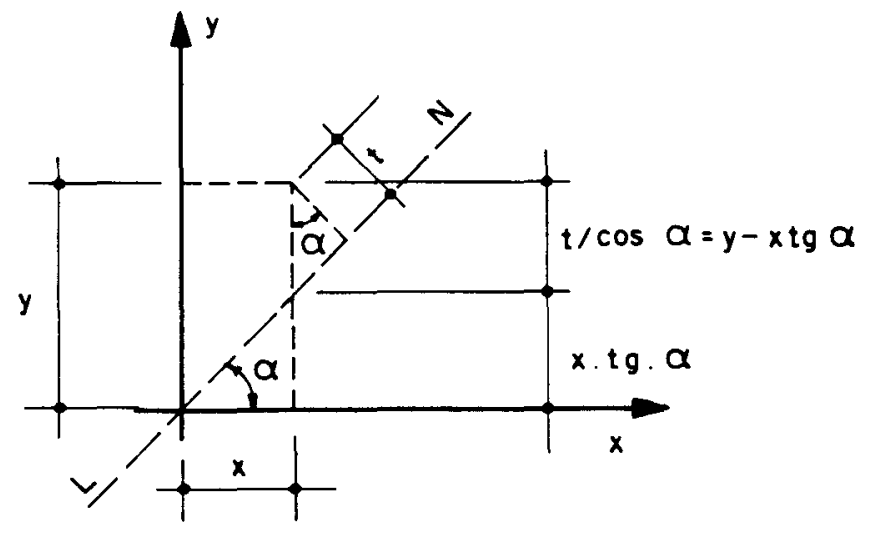

Fig. 3.5 - Distância de um ponto à linha neutra.

Novamente, de 3.10:

$$
\frac{\operatorname{sen} \alpha}{\cos \alpha}=-\frac{I_{x} \cdot \cos \alpha_{0}}{I_{y} \cdot \operatorname{sen} \alpha_{0}}
$$

$\frac{\operatorname{sen} \alpha_{0}}{I_{x} \cdot \cos \alpha}=-\frac{\cos \alpha_{0}}{I_{y} \cdot \operatorname{sen} \alpha_{0}}$


Com esta expressão e com a 3.11, tem-se:

$$
\begin{aligned}
& \frac{\sigma}{\text { M.t }}=\frac{\operatorname{sen} \alpha_{0}}{I_{x} \cdot \cos \alpha}=-\frac{\cos \alpha_{0}}{I_{y} \cdot \operatorname{sen} \alpha_{0}} \\
& \frac{\sigma}{M \cdot t}=\frac{\operatorname{sen} \alpha_{0} \cdot \cos \alpha}{I_{x} \cdot \cos ^{2} \alpha}=-\frac{\cos \alpha_{0} \cdot \operatorname{sen} \alpha}{I_{y} \cdot \operatorname{sen}^{2} \alpha} \\
& \frac{\sigma}{M \cdot t}=\frac{\operatorname{sen} \alpha_{0} \cdot \cos \alpha-\cos \alpha_{0} \cdot \operatorname{sen} \alpha}{I_{x} \cdot \cos ^{2} \alpha+I_{y} \cdot \operatorname{sen}^{2} \alpha} \\
& \frac{\operatorname{sen}\left(\alpha_{0}-\alpha\right)}{M . t}=\frac{I_{L N}}{M . \operatorname{sen}\left(\alpha_{0}-\alpha\right)} \\
& \frac{W_{L N}}{\sigma=}
\end{aligned}
$$

A expressão 3.12 fornece as tensões na seção, tendo como referência o eixo correspondente à linha neutra.

\subsubsection{FLEXÃO NORMAL COMPOSTA}

Além do momento fletor (cujo plano de ação contém um dos eixos centrais de inércia) e da força cortante, atua na seção uma força normal $\mathbf{N}$, conforme indicado na fig. 3.6.

As tensões normais originadas por $\mathbf{M}$ e $\mathbf{N}$ são somadas algebricamente e a expressão para a obtenção de seus valores é:

$$
\sigma=\frac{\mathrm{N}}{\mathrm{A}}+\frac{\mathrm{M}}{\mathrm{W}}
$$




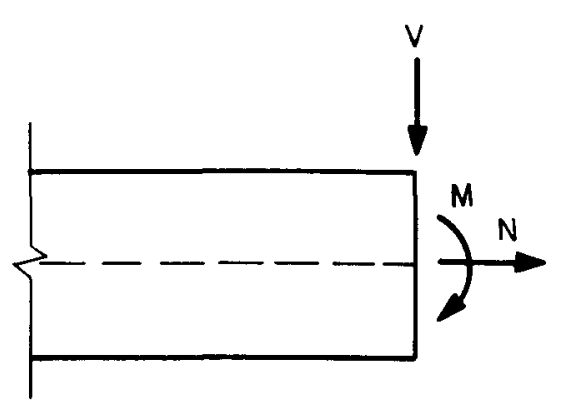

Fig. 3.6 - Esforços solicitantes na flexão normal composta.

Podem ser originadas tensões de tração ou de compressão na seção transversal. A linha neutra não será mais coincidente com o eixo $\mathbf{x}$ da seção (ver fig. 3.3), como na flexão normal simples, mas será paralela a este eixo. De 3.13 obtém-se a expressão que fornece a distância da linha neutra ao eixo $\mathbf{x}$, fazendo $\sigma=\mathbf{0}$ :

$$
\begin{aligned}
& \sigma=\frac{N}{A}+\frac{M}{I_{x}} y=0 \\
& y=-\frac{N \cdot I_{x}}{A \cdot M} \\
& y=-\frac{I_{x}}{A \cdot e}
\end{aligned}
$$

onde:

$$
e=\frac{M}{N}
$$

Maiores detalhes serão apresentados na seção 3.4 . 


\subsubsection{FLEXÃO OBLÍQUA COMPOSTA}

Os esforços solicitantes são os mesmos da flexão normal composta, sendo que o plano de ação do momento fletor não contém nenhum dos eixos centrais de inércia. Somando algebricamente as tensões normais devidas aos esforços solicitantes, tem-se (ver expressão 3.12):

$$
\sigma=\frac{N}{A}+\frac{M \cdot \operatorname{sen}\left(\alpha_{0}-\alpha\right)}{W_{L^{\prime} N^{\prime}}}
$$

onde L'N' (ver fig. 3.7) forma um ângulo $\alpha$ com o eixo $\mathbf{x}$ (se a flexão fosse simples, L'N' seria a linha neutra da seção).

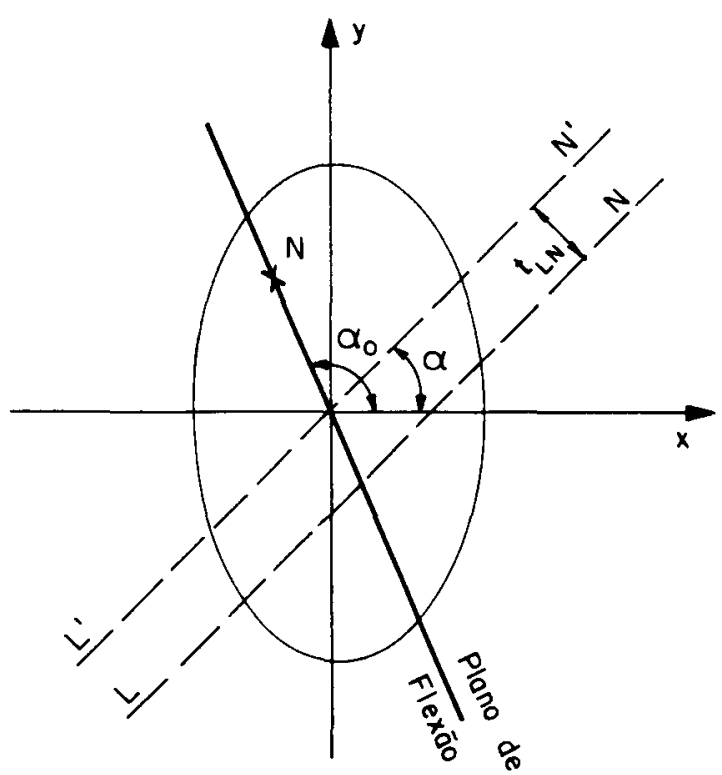

(a)

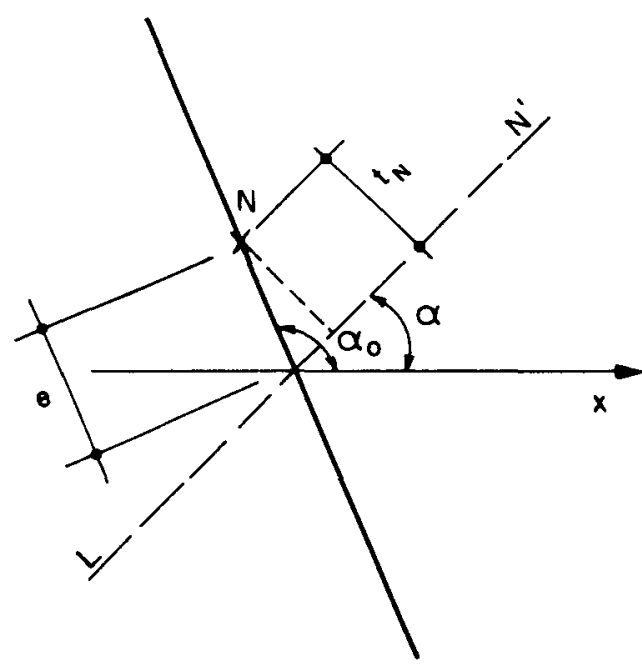

(b)

Fig. 3.7 - Posição da linha neutra na flexão composta oblíqua.

A linha neutra $(\mathrm{LN})$ será paralela à reta $\mathbf{L}^{\prime} \mathbf{N}^{\prime}$, distando $\mathbf{t}_{\mathrm{LN}}$ desta. Da fig. 3.7b, tomando-se a distância dos pontos de aplicação da força $\mathbf{N}\left(t_{N}\right)$ em relação a $L^{\prime} N^{\prime}$, e da expressão 3.15 , tem-se:

$$
\begin{aligned}
& M=N \\
& M=N \frac{t_{N}}{\operatorname{sen}\left(\alpha_{0}-\alpha\right)}
\end{aligned}
$$




$$
N=\frac{M \cdot \operatorname{sen}\left(\alpha_{0}-\alpha\right)}{t_{N}}
$$

De 3.16, em uma fibra distante t de L'N', tem-se:

$$
\begin{aligned}
& \sigma=\frac{N}{A}+\frac{M \cdot \operatorname{sen}\left(\alpha_{0}-\alpha\right)}{I_{L^{\prime} N^{\prime}}} t \\
& \sigma=\frac{N}{A}+\frac{N \cdot t_{N}}{I_{L^{\prime} N^{\prime}}} t \\
& \text { Para } t=t_{L N^{\prime}, \sigma=0:} \\
& 0=\frac{N}{A}+\frac{I_{L} \cdot t_{N}}{I_{L^{\prime}}} t_{L N} \\
& t_{L N}=-\frac{I_{L^{\prime} N^{\prime}}}{A \cdot t_{N}}
\end{aligned}
$$

\subsection{EXCENTRICIDADE DA FORÇA NORMAL}

A expressão 3.15 representa o valor da distância do ponto de aplicação da força normal $(\mathrm{N})$, relativa à origem dos eixos centrais de inércia, para que resulte o momento fletor (M).

Se o valor da excentricidade (e) conduzir à aplicação da força normal em um ponto localizado dentro do núcleo central de inércia da seção (pequena excentricidade), tem-se, em toda a seção transversal, tensões normais de tração ou de compressão, dependendo da natureza da força normal $(\mathrm{N})$; caso contrário (grande excentricidade), a seção transversal terá tensões normais de tração e de compressão e uma reta ao longo da qual as tensões serão nulas (linha neutra). 


\subsection{CONCEITOS DE INSTABILIDADE ELÁSTICA}

O deslocamento lateral de um elemento, por instabilidade elástica, ocorre na direção do menor raio de giração da seção transversal, quando atua uma força axial de compressão, levando o elemento à ruína com uma força menor do que a de ruptura do material à compressão. O elemento, a partir de um determinado valor da força de compressão $\left(\mathrm{N}_{\text {crii }}\right)$, abandona o regime de equilíbrio estável (peça indeformada) e passa ao regime de equilibrio instável, onde as flechas aumentam para valores praticamente constantes da força axial.

A determinação da força crítica (Ncrít) pode ser feita pelo método do equilíbrio (determinação do valor da força axial para que seja mantida uma configuração deformada de equilíbrio) ou pelo método energético (baseado nos deslocamentos sofridos, no trabalho realizado pela força axial e pela energia de deformação da barra).

\subsubsection{BARRAS PRISMÁTICAS}

Considerando uma barra reta (fig. 3.8), constituída por um material que obedeça à lei de Hooke e na qual se verifique a hipótese de Bernoulli, tem-se a expressão simplificada da linha elástica (ver seção 4.1):

$$
\frac{d^{2} y}{d x^{2}}=-\frac{M}{E . I}
$$

Sendo $\mathbf{k}^{2}=\mathbf{N} / \mathbf{E I}$, a solução da equação (3.19) resulta:

$$
y=C_{1} \cdot \operatorname{sen} k x+C_{1} \cdot \cos k x
$$

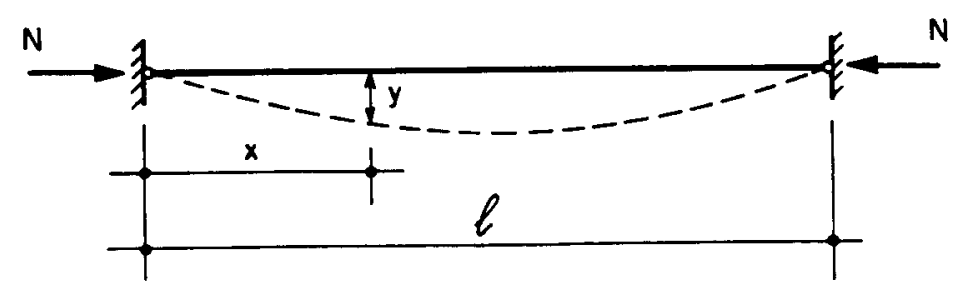

Fig. 3.8 - Barra prismática sujeita a força axial. 
Estabelecendo as condições de contorno e substituindo em 3.20:

- para $x=0 \quad \rightarrow \quad y=0$

- para $\mathrm{x}=l \quad \rightarrow \quad \mathrm{y}=0$

obtém-se $-\operatorname{sen} \mathbf{k} \boldsymbol{l}=\mathbf{0}$, condição que se verifica para $\mathbf{k} \boldsymbol{l}=\pi$ (menor valor positivo) e que resulta:

$$
\mathrm{N}_{\mathrm{fl}}=\pi^{2} \frac{\mathrm{E} . \mathrm{I}}{l^{2}}
$$

As forças críticas para outros tipos de vinculação podem ser encontradas de forma análoga e utilizando também a expressão da rotação em um ponto da barra:

$$
\phi=d y / d x=C_{1} \cdot k \cdot \cos k x-C_{2} \cdot \operatorname{sen} k x
$$

Normalmente, toma-se como caso fundamental a barra biarticulada (fig. 3.8), usando-se para os demais casos os comprimentos de flambagem (fig. 3.9), passando a expressão 3.21 para a forma geral:

$$
\mathrm{N}_{\mathrm{fl}}=\pi^{2} \frac{\mathrm{E} . \mathrm{I}}{l_{\mathrm{f} 1}}
$$

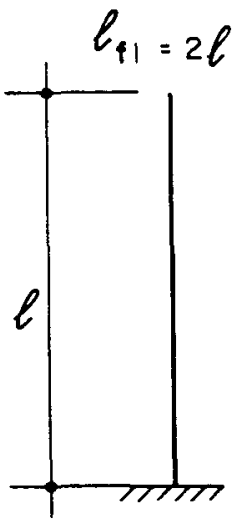

(a)

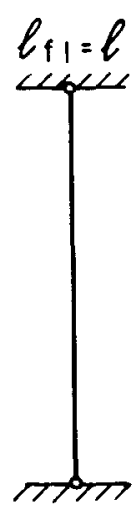

(b)

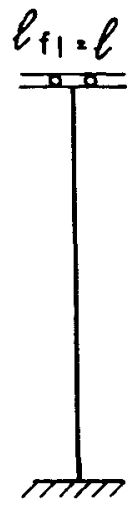

(c)

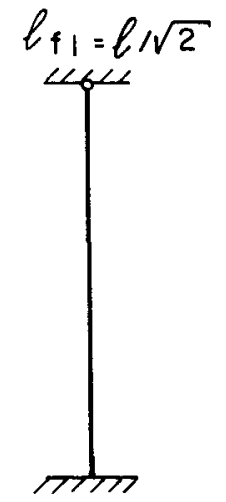

(d)

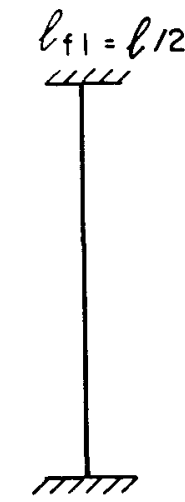

(e)

Fig. 3.9-Comprimentos de flambagem. 
As barras sujeitas à ação de forças axiais podem romper por instabilidade (peças esbeltas) ou por esmagamento do material de que são feitas (peças curtas). A classificação destes elementos é realizada com base no índice de esbeltez da peça,

$$
\lambda=\frac{l_{\mathrm{fl}}}{\mathrm{i}}
$$

onde i é o raio de giração da peça na direção considerada.

\subsubsection{BARRAS NÃO PRISMÁTICAS}

Se a barra considerada tiver a seção transversal variável, o valor da força crítica é obtido de forma análoga à apresentada na seção 3.5.1, sendo $\mathbf{I}=\mathbf{f}(\mathbf{x})$, resultando (LANGENDONCK, 1959).

$$
\mathrm{N}_{\mathrm{fl}}=\pi^{2} \frac{\mathrm{E} \cdot \mathrm{I}_{\mathrm{fl}}}{l_{\mathrm{fl}}^{2}}
$$

onde $\mathbf{I}_{\mathbf{n}}$ (momento de inércia de flambagem - momento de inércia "ficticio" da seção transversal em relação ao eixo que passa no centro de gravidade, perpendicularmente à direção de flambagem) é uma constante. Para os casos de vinculação $b, c, d$, e $e$ da fig. 3.9, sendo as barras troncônicas ou tronco prismáticas, o comprimento de flambagem é igual ao das barras prismáticas e o momento de inércia de flambagem é igual à média geométrica dos momentos de inércia das seções do topo e da base:

$$
\mathrm{I}_{f l}=\sqrt{\mathrm{I}_{\text {topo }} \cdot \mathrm{I}_{\text {base }}}
$$

Para os demais casos, ver o anexo I. O índice de esbeltez é dado pela expressão 3.24 , com

$$
i=\sqrt{\frac{\mathrm{I}_{\mathrm{fl}}}{\mathrm{S}_{\min }}}
$$

onde $\mathrm{S}_{\min }$ é a menor área das seções transversais da barra. 


\section{ESTUDO DAS DEFORMAÇÕES}

Serão abordadas, neste capítulo, as deformações transversais que ocorrem em elementos sujeitos a flexão. As deformações ocorridas no aço e no concreto, quando sujeitos a tensões, serão apresentadas sob a forma de diagramas tensão-deformação $(\sigma-\varepsilon)$, de acordo com a Norma Brasileira NBR-6118/1978 e o Código Modelo do Comité Euro-Internacional du Béton CEB-1990.

São também apresentadas as deformações de uma seção de concreto armado no estado limite último, conforme a já citada NBR-6118/1978.

\subsection{DEFORMAÇÃO POR FLEXÃO}

Considera-se um elemento estrutural reto, cujo eixo está representado na fig. 4.1, submetido a um carregamento que ocasiona compressão nas fibras superiores e tração nas fibras inferiores. Os pontos pertencentes ao eixo do elemento, sob a ação do carregamento, sofrem deslocamento e o eixo toma a forma curva (representada pela linha tracejada), caracterizando com a sua posição deformada a linha elástica do elemento: $\mathbf{r}$ representa o raio de curvatura da linha elástica.

Isolando-se um segmento genérico do elemento estrutural, indicado na fig. 4.2, desprezando-se os deslocamentos axiais e partindo-se da hipótese que o material obedeça a lei de Hooke, além das hipóteses de cálculo citadas na seção 3.1, a posição dos vértices da seção após a flexão é a dos vértices A-B-C-D: 


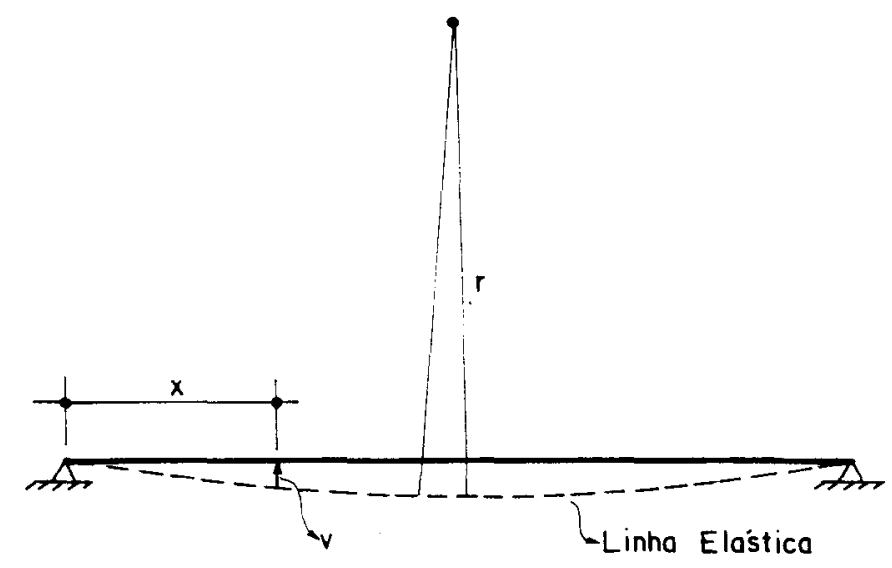

Fig. 4.1 - Linha elástica.

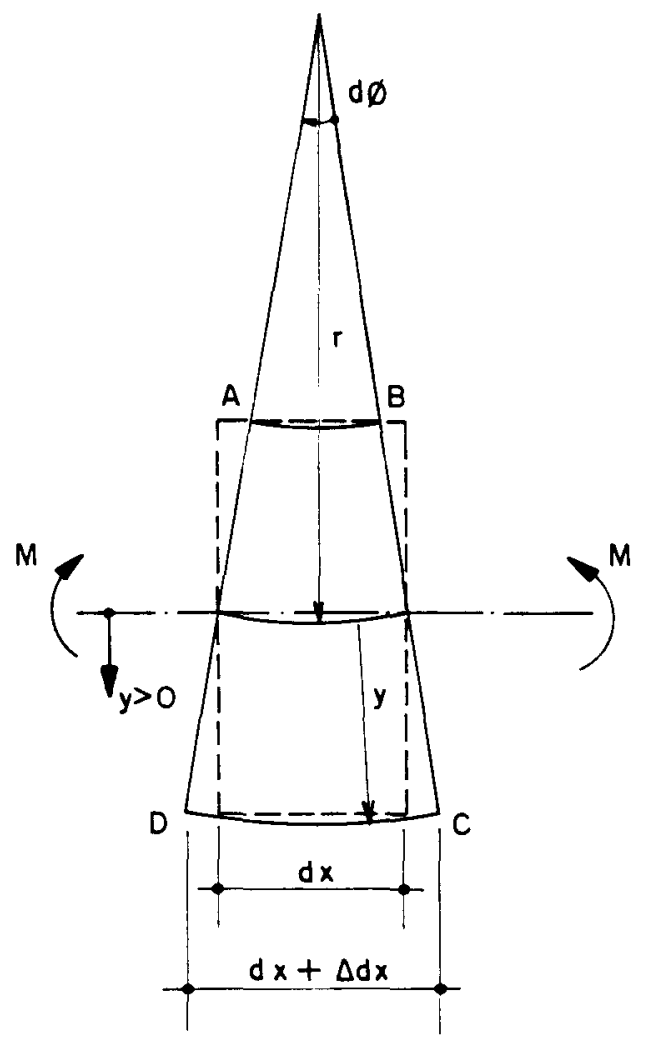

Fig. 4.2 - Curvatura proveniente de flexão. 
Lembrando que a deformação específica $(\varepsilon)$ é a razão entre a variação de comprimento e o comprimento inicial do elemento e que o módulo de elasticidade é a razão entre a tensão a que está sujeita uma fibra e a sua deformação específica, tem-se:

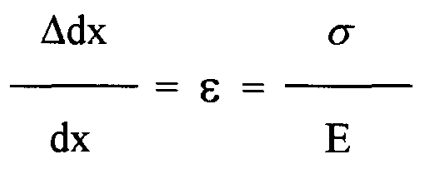

Sendo $\sigma=(M / I) \cdot y$, resulta:

$$
\Delta d x=\frac{M \cdot d x}{E \cdot I} y
$$

que fornece o alongamento $(\Delta \mathrm{dx})$ de uma fibra distante $\mathbf{y}$ do eixo do elemento. Da figura 4.2 e da expressão 4.1 , tem-se:

$$
\begin{aligned}
& \mathrm{d} \phi=\frac{\mathrm{dx}}{\mathrm{r}}=\frac{\Delta \mathrm{dx}}{\mathrm{y}}=\frac{\mathrm{M}}{\mathrm{E.I}} \mathrm{dx} \\
& \frac{1}{\mathrm{r}}=\frac{\mathrm{M}}{\mathrm{E} . \mathrm{I}}
\end{aligned}
$$

que é a curvatura do elemento genérico.

A expressão exata da curvatura é obtida pelo cálculo diferencial. Chamando a curvatura (1/r) de $k$, tem-se:

$$
\mathrm{k}=\frac{\frac{\mathrm{d}^{2} \mathrm{v}}{\mathrm{dx}^{2}}}{\left[1+\left(\frac{\mathrm{dv}}{\mathrm{dx}}\right)^{2}\right]^{3 / 2}}
$$


No caso de pequenos deslocamentos (teoria de $1^{\mathrm{a}}$ ordem), o termo $(\mathrm{dv} / \mathrm{dx})^{2}$ é muito pequeno em relação à unidade e, portanto, pode ser desprezado. Desta forma, tem-se uma equação simplificada da curvatura:

$$
k=\frac{d^{2} v}{d x^{2}}
$$

De 4.4 e 4.2 , tem-se:

$$
\frac{d^{2} v}{d x^{2}}=\frac{M}{E . I}
$$

A expressão 4.5 não é válida para materiais que não possuam linearidade física (relação linear entre as tensões e deformações). A linearidade fisica é relativa ao material de que é constituído o elemento estrutural. A linearidade entre as deformações ocorridas em uma estrutura, ou em um de seus elementos, e as forças nela aplicadas é caracterizada como linearidade geométrica, sendo possível neste caso a aplicação do princípio da superposição dos efeitos para análise dos efeitos das forças às quais está sujeita a estrutura (teoria de $1^{\underline{a}}$ ordem).

\subsection{ANALOGIA DE MOHR}

É um processo destinado à determinação dos deslocamentos (v), em relação à posição inicial, dos pontos dos eixos das barras fletidas. Foi desenvolvido a partir da semelhança entre a equação diferencial simplificada da linha elástica (4.5) e a equação diferencial da estática (4.6); da derivada dos momentos em relação a x (4.7) e da derivada dos deslocamentos em relação a x (4.8). Da semelhança entre estas equações diferenciais, concluiu-se haver analogia entre os momentos fletores (M) e os deslocamentos (v), entre a força cortante (Q) e os deslocamentos angulares $(\phi)$ e entre a força imposta ao elemento (q) e a razão $\mathrm{M} / \mathrm{EI}$.

$$
\frac{d^{2} M}{d x^{2}}=q
$$




$$
\begin{aligned}
& \frac{\mathrm{dM}}{\mathrm{dx}}=\mathrm{Q} \\
& \frac{\mathrm{dv}}{\mathrm{dx}}=\phi
\end{aligned}
$$

A analogia de Mohr considera os deslocamentos ( $\mathrm{v}$ ou y) como sendo o momento fletor em uma barra equivalente, carregada com uma força distribuída cuja taxa de distribuição é $\mathrm{M} / \mathrm{EI}$, onde $\mathbf{M}$ é o momento fletor atuante na barra dada.

O momento fletor $\mathbf{M}$ será considerado positivo quando tracionar as fibras inferiores e comprimir as fibras superiores da seção na qual está aplicado. $O$ valor da força $\mathbf{q}$ será positivo, aplicado verticalmente de cima para baixo, quando $\mathbf{M}$ for positivo.

Empregando-se este processo, são feitas as seguintes analogias:

- a razão $\mathbf{M} / \mathbf{E I}$ referente à barra dada fornece a força distribuída $\mathbf{q}$ da barra conjugada;

- as forças cortantes $\mathbf{Q}$ calculadas na barra conjugada, proveniente do carregamento $q$, fornecem as rotações da tangente à elástica $\phi$ na barra dada;

- os momentos fletores $\mathbf{M}$ calculados na barra conjugada, provenientes da força $\mathbf{q}$, fornecem o deslocamento vertical y na barra dada.

A determinação das vinculações da barra conjugada depende das vinculações da barra dada. Com base nas analogias apresentadas, se houver um apoio simples (fig. 4.3), onde o deslocamento vertical é nulo $(\mathrm{y}=0)$ e a rotação é livre $(\phi \neq 0)$, deve-se ter um apoio na barra conjugada para o qual o momento seja nulo (pois o deslocamento vertical na barra dada é nulo) e a força cortante não seja nula (a rotação angular é permitida no apoio da barra dada). Neste caso, o apoio da barra conjugada será o mesmo apoio da barra dada. A tabela 4 .1 apresenta a transformação dos vínculos para a barra conjugada (SÜSSEKIND, 1987). 


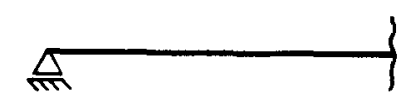

Fig. 4.3 - Apoio simples.

Tabela 3.1 - Transformação de vínculos para barra conjugada.

\begin{tabular}{|c|c|c|}
\hline barra dada & barra conjug & cond contomo \\
\hline$\stackrel{A}{=}$ & $\stackrel{A}{S}$ & $\begin{array}{l}y_{A}=0 \\
\phi_{A} \neq 0\end{array}$ \\
\hline 争 & A & $\begin{array}{l}y_{A}=0 \\
\varnothing_{A}=0\end{array}$ \\
\hline$A$ & A & $\begin{array}{l}y_{A} \neq 0 \\
\varnothing_{A} \neq 0\end{array}$ \\
\hline$\frac{A}{\frac{\mathrm{d}}{\mathrm{f}}}$ & $A$ & $\begin{array}{l}y=0 \\
\varnothing_{A}^{\text {esq }}=\varnothing_{A}^{\text {dir }}\end{array}$ \\
\hline A & $\vdash \frac{A}{\text { 点 }}$ & $\begin{array}{l}y \neq 0 \\
\emptyset_{A} \text { esq } \neq \varnothing_{A} \mathrm{dir}\end{array}$ \\
\hline$\vdash \frac{A}{\underline{8}}$ & $\frac{A}{\frac{8}{m}}$ & $\begin{array}{l}y=0 \\
\emptyset_{A}^{\text {esq }} \neq \varnothing_{A}^{\text {dir }}\end{array}$ \\
\hline
\end{tabular}

A barra conjugada de uma barra isostática será isostática. A barra conjugada de uma barra hiperestática, sem recalques de apoios, será hipostática; neste caso o seu carregamento (M/EI) propiciará o auto-equilíbrio da barra. Este método aplica-se às barras de inércia variável, porém resulta da analogia $\mathrm{q}=\mathrm{M} / \mathrm{EI}$ um carregamento mais complexo do que o obtido para uma barra de seção constante.

\subsection{DEFORMAÇÕES NO CONCRETO}

A Norma Brasileira NBR-6118/1978 admite o uso de um diagrama simplificado de tensão-deformação do concreto, fig. 4.4, para a determinação da resistência à compressão de projeto. O diagrama é composto de uma parábola de $2^{\underline{Q}}$ 
grau, que passa pela origem e tem seu vértice no ponto de abcissa (deformação) 0,002 e ordenada (tensão) $0,85 \mathrm{f}_{\mathrm{cd}}$, e de uma reta paralela ao eixo das deformações, compreendida entre as abcissas 0,002 e 0,0035 e de ordenada $0,85 f_{c d}$. A resistência à tração do concreto é desprezada no estado limite último. Para os trechos $\mathrm{OA}$ e $\mathrm{AB}$ da fig. 4.4 , considerando-se positivos os encurtamentos, tem-se:

- trecho OA $\left(0 \leq \varepsilon_{\mathrm{c}}<\varepsilon_{\mathrm{cl}}\right)$

$$
\sigma_{\mathrm{c}}=0,85 \mathrm{f}_{\mathrm{cd}}\left[2\left(\frac{\varepsilon_{\mathrm{c}}}{\varepsilon_{\mathrm{cl}}}\right)-\left(\frac{\varepsilon_{\mathrm{c}}}{\varepsilon_{\mathrm{cl} 1}}\right)^{2}\right]
$$

- trecho $A B\left(\varepsilon_{\mathrm{cl}} \leq \varepsilon_{\mathrm{c}}<\varepsilon_{\mathrm{cu}}\right)$

$$
\sigma_{\mathrm{c}}=0,85 \mathrm{f}_{\mathrm{od}}
$$

onde:

$$
\begin{aligned}
& \varepsilon_{\mathrm{cl}}=0,002 \\
& \varepsilon_{\mathrm{cu}}=0,0035
\end{aligned}
$$

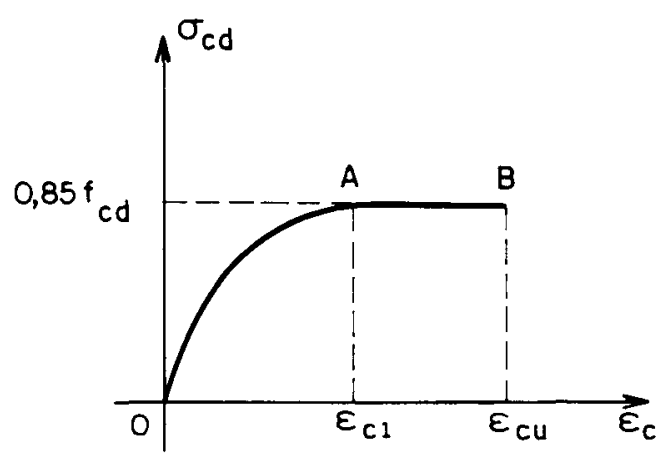

Fig. 4.4 - Diagrama simplificado $(\sigma-\varepsilon)$ para o concreto.

A fig. 4.5 mostra a forma genérica do diagrama tensão-deformação do concreto (Boletim $n^{\circ}$ 203, CEB-1990), onde é utilizado o valor médio para a resistência à compressão do concreto $\left(\mathrm{f}_{\mathrm{cm}}\right)$.

Os valores de $\mathbf{f}_{\mathbf{c}}, \mathbf{E}_{\mathbf{c} 1}$ e $\mathbf{E}_{\mathbf{c}}$ a serem utilizados (médios, característicos ou de cálculo) devem ser adequados ao tipo de análise que está sendo efetuada, ou seja, à 
consideração dos efeitos advindos da não-linearidade física e da não linearidade geométrica bem como aos estados limites a serem verificados (FRANÇA, 1991; CEB-1990):

- para análises não-lineares (apenas não linearidade física dos materiais), em serviço ou estado limite último, utilizam-se os valores médios da resistência à compressão $\left(f_{\mathrm{cm}}\right)$ e módulo de elasticidade $\left(E_{c}\right)$ obtido pela expressão 4.12 ;

- para análises não lineares de segunda ordem (não-linearidade física e geométrica) para obtenção das deformações e esforços resultantes, utilizam-se os valores característicos com os adequados coeficientes de minoração $\left(\gamma_{\mathrm{c}}=\gamma_{\mathrm{E}}=1,2-\right.$ para combinações fundamentais - itens 1.6.3.4 e 6.6.2.3 do CEB-1990);

- para análise da capacidade resistente das seções críticas, utilizam-se coeficientes de minoração com valores iguais a 1,5 (valor usual).

O valor da tensão de compreensão do concreto, e também de seu módulo de elasticidade, ainda sofrem influência de um coeficiente $(\alpha)$ utilizado para levar em conta os efeitos das ações de longa duração, resistência na idade do evento considerado e temperatura do concreto, sendo o valor usualmente utilizado igual a 0,85, proposto por Rüsch e adotado no diagrama parábola-retângulo. O CEB-1990 (boletins 195, 196 e 198) fornece parâmetros para a determinação dos coeficientes $\alpha$ e $\alpha_{\mathrm{EC}}$ em função da idade e temperatura do concreto. O EUROCODE-2 recomenda $\alpha$ e $\alpha_{\mathrm{EC}}=1,0$ para as análises não-lineares em serviço ou estado limite último e para análises não-lineares de $2^{\underline{a}}$ ordem, recomendando $\alpha=0,85$ para a análise da capacidade portante de seções críticas, utilizando-se o diagrama parábola-retângulo.

Os valores de cálculo serão tratados com mais detalhes no capítulo 8.

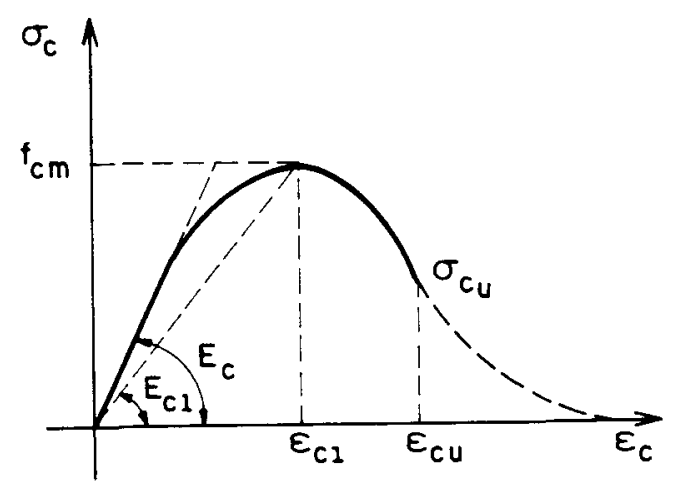

Fig. 4.5 - Diagrama $(\sigma-\varepsilon)$ para o concreto - CEB 1990. 
A relação $\sigma-\varepsilon$ pode ser obtida pela seguinte função, válida para $\left|\varepsilon_{\mathrm{c}}\right|<\left|\varepsilon_{\mathrm{cu}}\right|$ :

$$
\sigma_{\mathrm{c}}=-\frac{\frac{\mathrm{E}_{\mathrm{c}}}{\mathrm{E}_{\mathrm{cl}}} \cdot \frac{\varepsilon_{\mathrm{c}}}{\varepsilon_{\mathrm{cl}}}-\left(\frac{\varepsilon_{\mathrm{c}}}{\varepsilon_{\mathrm{cl}}}\right)^{2}}{1+\left(\frac{\mathrm{Ec}}{\mathrm{E}_{\mathrm{cl}}}-2\right) \frac{\varepsilon_{\mathrm{c}}}{\varepsilon_{\mathrm{cl}}}} \mathrm{f}_{\mathrm{cm}}
$$

na qual:

$\mathrm{E}_{\mathrm{c}}=$ módulo de elasticidade do concreto

$\sigma_{\mathrm{c}}=$ tensão de compressão $(\mathrm{MPa})$

$\varepsilon_{\mathrm{c}}=$ deformação à compressão do concreto

$\varepsilon_{\mathrm{cl}}=0,0022$ (encurtamento)

$E_{\mathrm{cl}}=(\mathrm{fcm} / 0,0022)$ módulo de elasticidade secante no ponto de deformação $\varepsilon_{\mathrm{cl}}$

O módulo de elasticidade é dado por:

$$
E_{c}=\alpha_{E}\left[f_{c m} / f_{c m o}\right]^{1 / 3}
$$

onde:

$\alpha_{\mathrm{E}}=2,15 \times 10^{4} \mathrm{MPa}$

$\mathrm{f}_{\mathrm{cmo}}=10 \mathrm{MPa}$

$\mathrm{f}_{\mathrm{cm}}=\mathrm{f}_{\mathrm{ck}}+8 \mathrm{MPa}$

Para a parte descendente do diagrama $\sigma-\varepsilon$ do concreto, a equação 4.11 só é válida para valores de $\left|\sigma_{\mathrm{c}}\right| / \mathrm{f}_{\mathrm{cm}} \geq 0,5$. A deformação $\varepsilon_{\mathrm{cu}}$ não tem outro significado a não ser o limite de aplicação da equação $4.11 \mathrm{e}$, para uma tensão de compressão igual à metade do valor de $\mathbf{f}_{\mathbf{c m}}$, pode ser calculada pela expressão:

$$
\varepsilon_{\mathrm{cu}}=\varepsilon_{\mathrm{cl}}\left\{\frac{1}{2}\left(\frac{\mathrm{E}_{\mathrm{c}}}{2 . \mathrm{E}_{\mathrm{cl}}}+1\right)+\left[\frac{1}{4}\left(\frac{\mathrm{E}_{\mathrm{c}}}{2 . \mathrm{E}_{\mathrm{cl}}}+1\right)^{2}-\frac{1}{2}\right]^{1 / 2}\right\}
$$




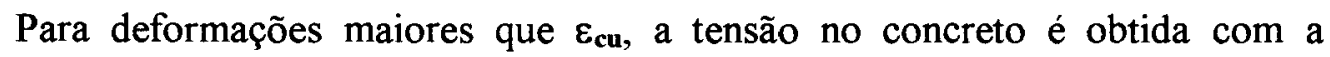
expressão:

$$
\begin{aligned}
\sigma_{\mathrm{c}}=- & {\left[\left(\frac{1}{\varepsilon_{\mathrm{cu}} / \varepsilon_{\mathrm{cl}}} \cdot \xi-\frac{2}{\left(\varepsilon_{\mathrm{cu}} / \varepsilon_{\mathrm{cl}}\right)^{2}}\right) \cdot\left(\frac{\varepsilon_{\mathrm{c}}}{\varepsilon_{\mathrm{cl}}}\right)^{2}+\right.} \\
& \left.\left(\frac{4}{\varepsilon_{\mathrm{cul}} / \varepsilon_{\mathrm{cl}}}-\xi\right) \cdot \frac{\varepsilon_{\mathrm{c}}}{\varepsilon_{\mathrm{cl}}}\right]^{-1} \cdot \mathrm{f}_{\mathrm{cm}}
\end{aligned}
$$

com:

$$
\xi=\frac{4\left[\left(\frac{\varepsilon_{\mathrm{cl}}}{\varepsilon_{\mathrm{cl}}}\right)^{2} \cdot\left(\frac{\mathrm{E}_{\mathrm{c}}}{\mathrm{E}_{\mathrm{cl}}}-2\right)+2 \cdot \frac{\varepsilon_{\mathrm{cl}}}{\varepsilon_{\mathrm{cl}}}-\frac{\mathrm{E}_{\mathrm{c}}}{\mathrm{E}_{\mathrm{cl}}}\right]}{\left[\frac{\varepsilon_{\mathrm{cl}}}{\varepsilon_{\mathrm{cl}}} \cdot\left(\frac{\mathrm{E}_{\mathrm{c}}}{\mathrm{E}_{\mathrm{cl}}}-2\right)+1\right]^{2}}
$$

O código modelo do CEB (Boletim $n^{\circ}$ 204, CEB-1990) admite o mesmo diagrama simplificado (fig. 4.4) adotado pela norma brasileira, empregando (devido às influências das cargas permanentes) o coeficiente 0,85 e a tensão constante para as deformações pertencentes ao intervalo entre $\varepsilon_{\mathrm{cl}}$ e $\varepsilon_{\mathrm{cu}}$, sendo $\varepsilon_{\mathrm{cl}}=0,002$. Na flexão tem-se:

$$
\varepsilon_{\mathrm{cu}}=\left\{\begin{array}{l}
0,0035 \text { para } \mathrm{f}_{\mathrm{ck}} \leq 50 \mathrm{MPa} \\
0,0035\left(50 / \mathrm{f}_{\mathrm{ck}}\right) \text { para } 50 \mathrm{MPa}<\mathrm{f}_{\mathrm{ck}} \leq 80 \mathrm{MPa}
\end{array}\right.
$$

Para compreensão axial:

$$
\varepsilon_{\mathrm{cu}}=0,002
$$


O CEB cita ainda outro diagrama simplificado, que é o diagrama de tensões uniformes. A tensão média é obtida por $\left(\mathrm{f}_{\mathrm{ck}} \mathrm{em} \mathrm{MPa}\right)$ :

- seções não fissuradas

$$
\mathrm{f}_{\mathrm{cd} 1}=0,85\left\{1-\frac{\mathrm{f}_{\mathrm{ck}}}{250}\right\} \mathrm{f}_{\mathrm{cd}}
$$

- seções fissuradas

$$
\mathrm{f}_{\mathrm{cd} 2}=0,60\left\{1-\frac{\mathrm{f}_{\mathrm{ck}}}{250}\right\} \mathrm{f}_{\mathrm{cd}}
$$

As expressões 4.16 e 4.17 são válidas sob a condição de que a deformação máxima ocorrida em qualquer ponto da seção não supere:

$$
\varepsilon_{\mathrm{cu}}=0,004-0,002 \frac{f_{\mathrm{ck}}}{100}
$$

No cálculo das deformações da estrutura ou de um de seus elementos, segundo o CEB-1990, o coeficiente de minoração da resistência do concreto $\left(\gamma_{c}\right)$ pode ser reduzido para 1,2 ou 1,0 (no caso de combinações raras). $E_{c s}$ é utilizado quando apenas a análise elástica é feita. É um módulo reduzido, a fim de levar em conta a deformação plástica inicial.

$$
\mathrm{E}_{\mathrm{cs}}=0.85 . \mathrm{E}_{\mathrm{c}}
$$

A utilização do diagrama parábola-retângulo (fig. 4.4) leva a estimativas exageradas das deformações. Sugere-se (FUSCO, 1986), para o cálculo das deformações de pilares utilizando diagramas força normal - momento fletor curvatura (maiores detalhes serão fornecidos no capítulo 6), confeccionados com base no diagrama de deformações do concreto, a utilização das curvas do digrama da fig. 4.6, sendo:

- curva B: pilares com índice de esbeltez $(\lambda)$ maior que 140; 
- curva C: pilares com $80 \leq \lambda \leq 140$;

- curva D: pilares com $\lambda<80$ e contraventados; este digrama utiliza o valor médio da resistência à compressão $\left(\mathrm{f}_{\mathrm{cm}}\right)$.

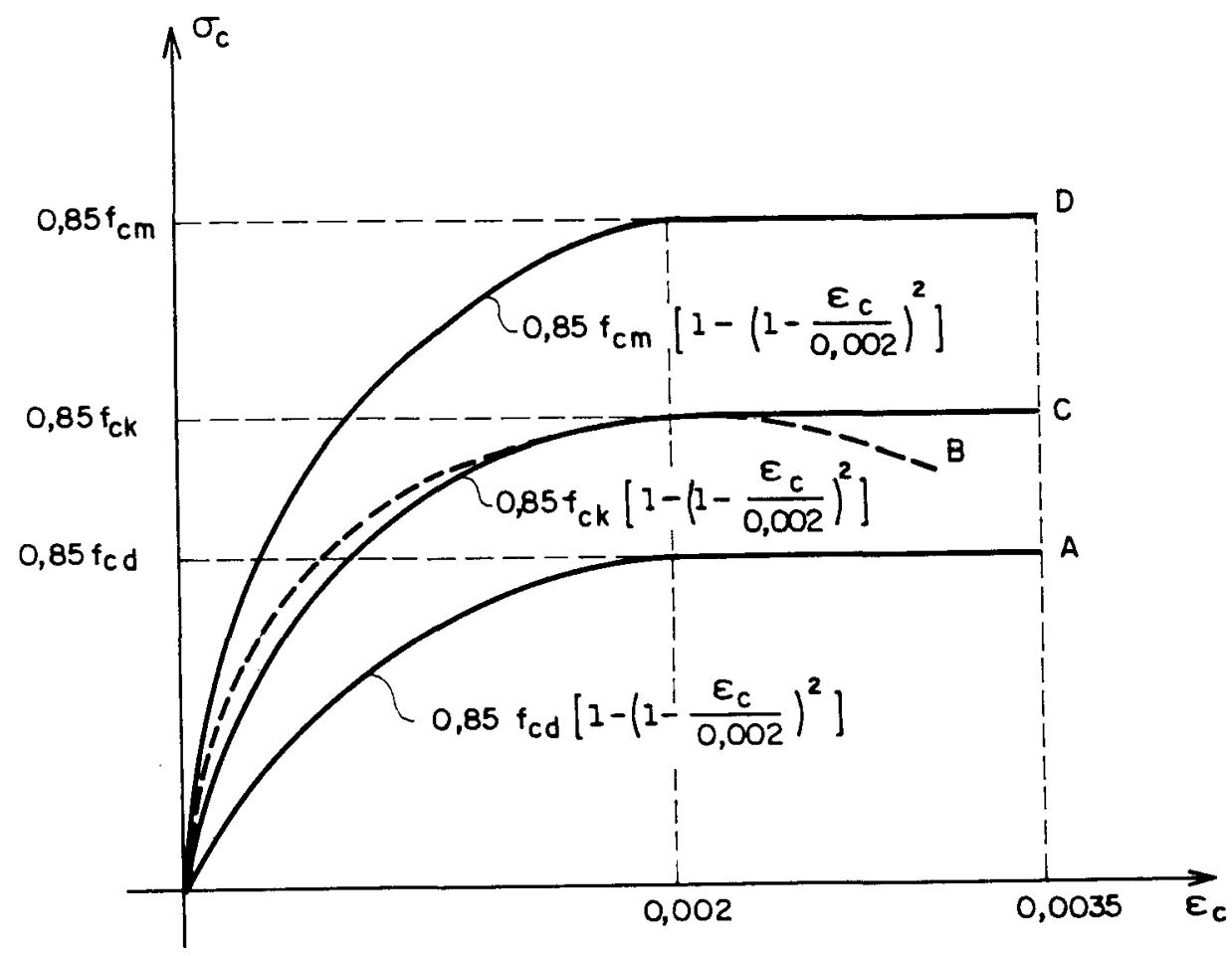

Fig. 4.6 - Possíveis diagramas $(\sigma-\varepsilon)$ para cálculo dos deslocamentos.

\subsection{DEFORMAÇÕES NO AÇO}

Para os aços de classe A, laminados a quente, a NBR-6118/1978 permite a adoção do diagrama tensão-deformação simplificado da fig. 4.7 , onde:

$$
f_{y d}=f_{y c d}=\frac{f_{y k}}{\gamma_{s}}
$$

Para os aços de classe B, encruados por deformação a frio, o diagrama adotado é o da figura 4.8. Há três trechos definidos:

$-1^{\circ}$ trecho: linear

$$
0 \leq \varepsilon_{\mathrm{s}} \leq \varepsilon_{0,7}
$$




$$
\sigma_{\mathrm{s}}=\varepsilon_{\mathrm{s}} . \mathrm{E}_{\mathrm{s}}
$$

- 2ำ trecho: parábola

$$
\begin{aligned}
& \varepsilon_{0,7}<\varepsilon_{\mathrm{s}}<\varepsilon_{\mathrm{yd}} \\
& \varepsilon_{\mathrm{s}}=\frac{\left|\sigma_{\mathrm{s}}\right|}{\mathrm{E}_{\mathrm{s}}}+\frac{1}{45}\left(\frac{\left|\sigma_{\mathrm{s}}\right|}{\mathrm{f}_{\mathrm{yd}}}-0,7\right)^{2}
\end{aligned}
$$

- 3ำ trecho: patamar

$$
\begin{aligned}
& \varepsilon_{\mathrm{yd}} \leq \varepsilon_{\mathrm{s}} \leq 0,01 \\
& \sigma_{\mathrm{s}}=\mathrm{f}_{\mathrm{yd}}
\end{aligned}
$$

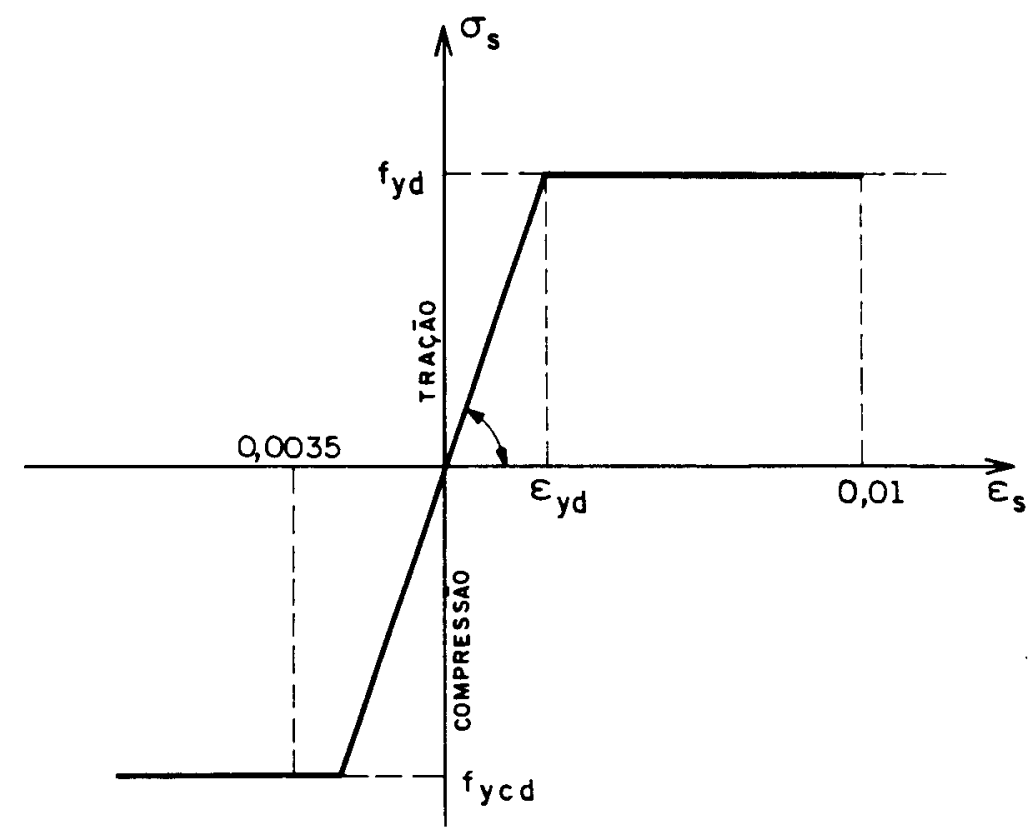

Fig. 4.7 - Diagrama $(\sigma-\varepsilon)$ de cálculo para aços classe A. 


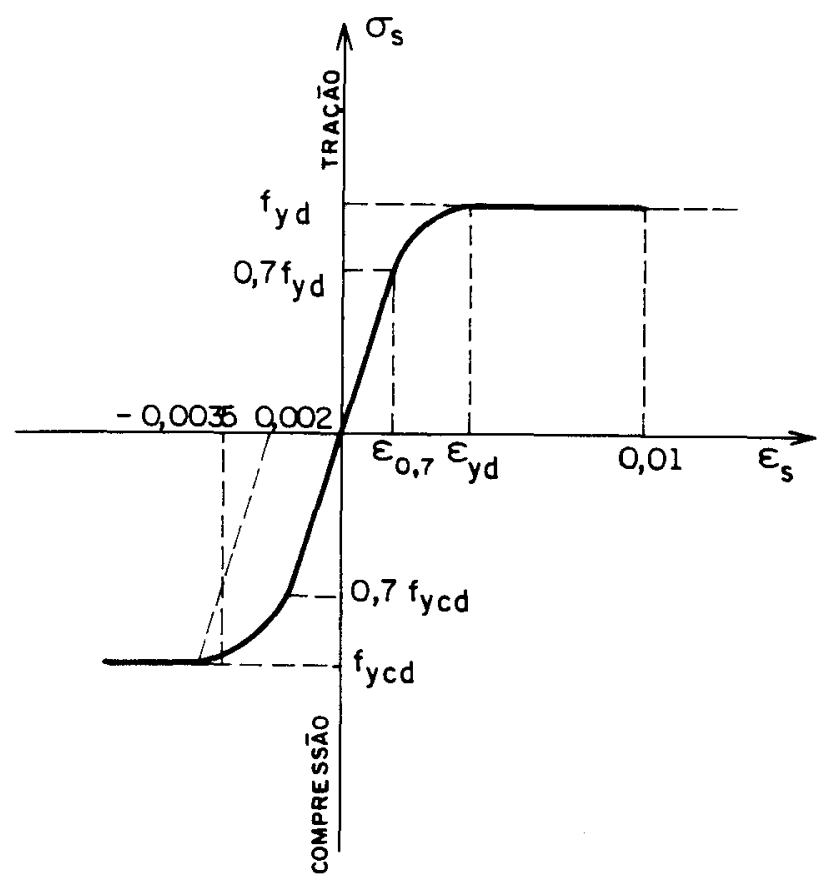

Fig. 4.8 - Diagrama $(\sigma-\varepsilon)$ de cálculo para aços classe B.

onde:

$f_{y d}=f_{y k} / \gamma_{s}$

$\varepsilon_{0,7}=0,7 . \mathrm{f}_{\mathrm{yk}} /\left(\gamma_{\mathrm{s}} . \mathrm{E}_{\mathrm{s}}\right)$

$\varepsilon_{y d}=f_{y k} /\left(\gamma_{s} . E_{s}\right)+0,002$

$E_{\mathrm{s}}=210.000 \mathrm{MPa}$ (módulo de elasticidade do aço)

\subsection{ESTADO LIMITE ÚLTIMO}

Caracteriza o esgotamento da capacidade resistente de peças submetidas a solicitação normais. Por convenção, ocorre quando há esmagamento (ruptura) do concreto ou deformação plástica excessiva da armadura tracionada.

A ruptura do concreto, por convenção, ocorre quando seu encurtamento alcança os valores de $3,5 \times 10^{-3}$ na flexão ou $2 \times 10^{-3}$ na compreensão axial. $O$ alongamento das armaduras é limitado a $10 \times 10^{-3} ; \varepsilon_{\mathrm{cd}}$ e $\varepsilon_{\mathrm{yd}}$ são as deformações 
específicas de cálculo para o concreto e para o aço, respectivamente. Estas deformações caracterizam os domínios de deformação, definidos pela Norma Brasileira NBR-6118/1978. O código modelo do CEB-1990 não apresenta valores limites para o encurtamento máximo do concreto quando se utiliza o diagrama da fig. 4.5 .

\subsubsection{DOMÍNIO 1}

O estado limite último é caracterizado pela deformação específica do aço: $\varepsilon_{s d}=0,01$ (fig.3.9). Neste domínio ocorre tração axial e tração excêntrica de pequena excentricidade. $O$ concreto é suposto inteiramente fissurado, sendo a seção resistente a seção das armaduras.

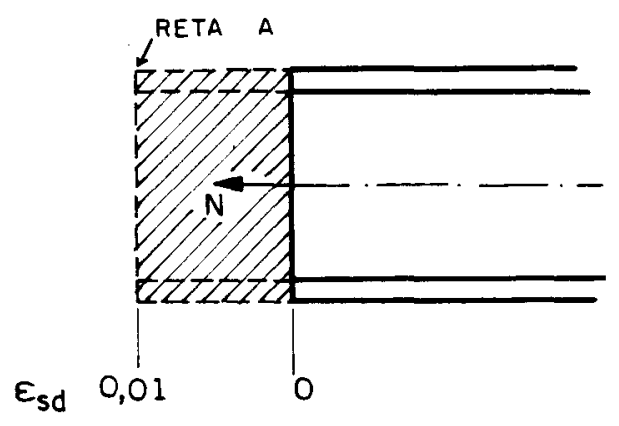

(a) Tração axial

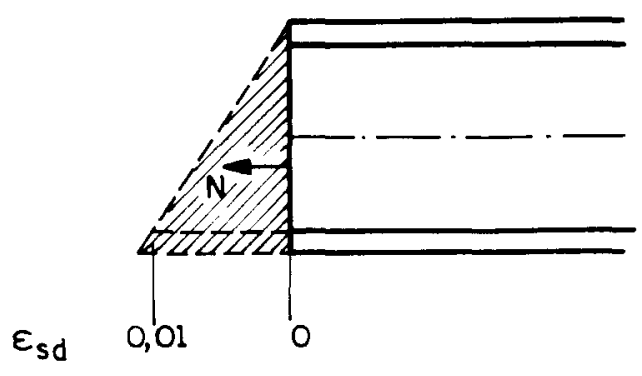

(b) Tração excêntrica

Fig. 4.9 - Deformações no domínio 1.

\subsubsection{DOMÍNIO 2}

O estado limite último é caracterizado pela deformação específica do aço: $\varepsilon_{\text {sd }}=0,01$ (fig. 3.10). Neste domínio ocorre tração excêntrica de grande excentricidade, compressão excêntrica de grande excentricidade e flexão simples. A deformação específica do concreto varia de 0,0 a 0,0035 . As armaduras de compressão podem ser consideradas apenas para a deformação específica do concreto variando entre 0,002 e 0,0035 . 


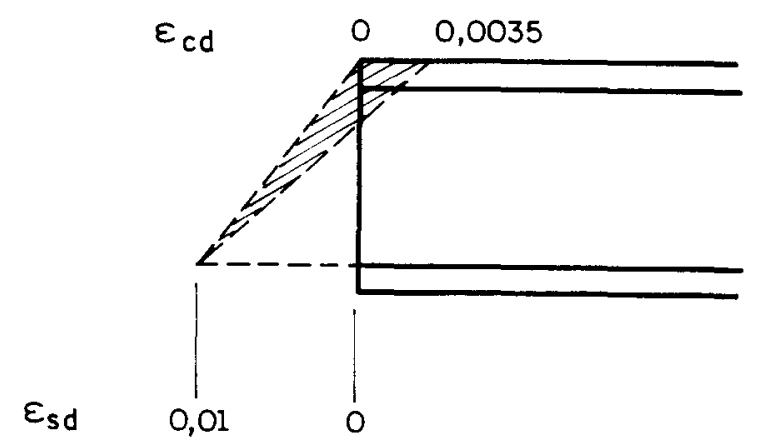

Fig. 4.10 - Deformação no domínio 2.

\subsubsection{DOMÍNIO 3}

O estado limite último é caracterizado pela deformação especifica do concreto: $\varepsilon_{\propto d}=0,0035$ (fig. 4.11). Neste domínio ocorre tração excêntrica de grande excentricidade, compressão excêntrica de grande excentricidade e flexão simples. São as peças subarmadas (ruptura do concreto ocorre simultaneamente com o escoamento da armadura). A deformação específica do aço varia de $\varepsilon_{y d}$ a 0,01 .

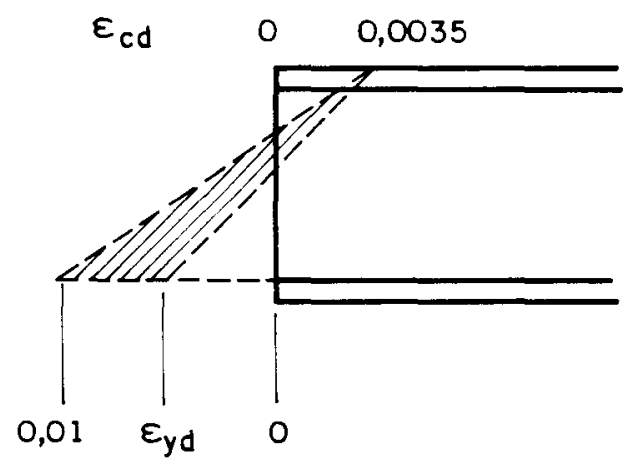

Fig. 4.11 - Deformação no domínio 3.

\subsubsection{DOMINIO 4}

O estado limite último é caracterizado pela deformação específica do concreto: $\varepsilon_{\propto d}=0,0035$ (fig. 3.12). Neste dominio ocorrem as mesmas solicitações dos 
domínios 2 e 3 . São as peças subarmadas (a ruptura do concreto ocorre sem que haja escoamento da armadura).

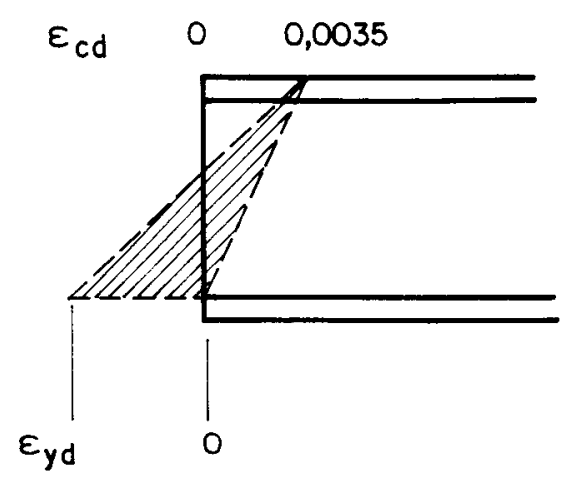

Fig. 4.12 - Deformações no domínio 4 .

\subsubsection{DOMÍNIO 4A}

O estado limite último é caracterizado pela deformação específica do concreto: $\varepsilon_{\propto d}=0,0035$. Ambas as armaduras estão comprimidas (fig. 3.13). Este domínio só é possível na compressão excêntrica com pequena excentricidade.

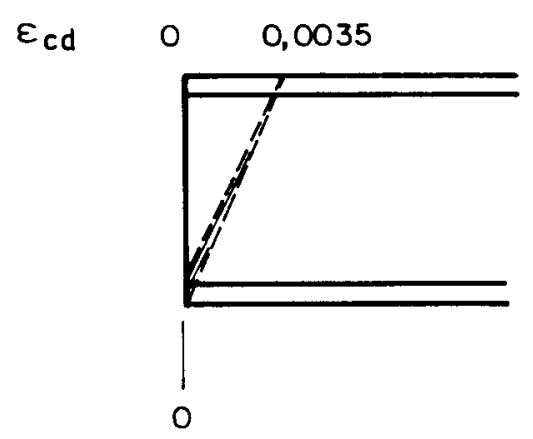

Fig. 4.13 - Deformações no domínio 4a.

\subsubsection{DOMÍNIO 5}

O estado limite último é caracterizado pela deformação específica do concreto: $\varepsilon_{\mathrm{cd}}=0,002$ a $(3 / 7) \mathrm{h}$ da borda mais comprimida, ocorrendo na compressão excêntrica com pequena excentricidade. 


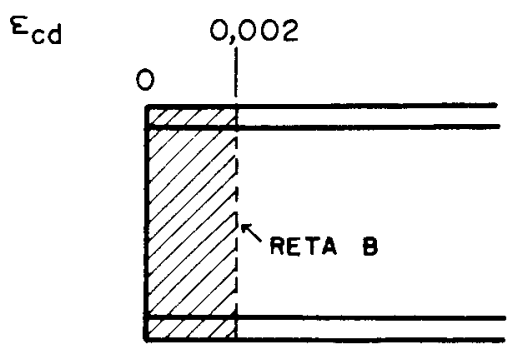

(a) Compressão axial

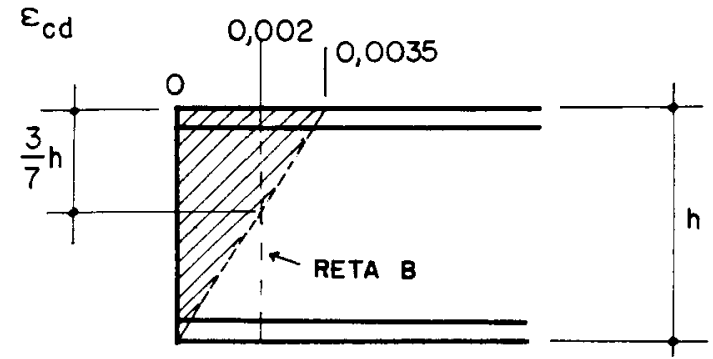

(b) Compressão excêntrica

Fig. 4.14 - Deformações no domínio 5. 


\section{INSTABILIDADE DE BARRAS}

Serão abordados neste capítulo alguns conceitos básicos sobre comportamento de barras sujeitas à compressão e à flexo-compressão e métodos para a obtenção das cargas críticas.

Estudos mais detalhados sobre a instabilidade de barras são encontrados em FUSCO (1986) e CEB/FIP (1978). Não serão abordadas, na determinação das cargas críticas, simplificações relativas ao processo do pilar padrão, suficientemente preciso apenas quando a barra é de seção transversal constante ao longo do comprimento, inclusive a armadura, e quando não existem cargas transversais aplicadas (FUSCO, 1986).

\subsection{CONCEITOS SOBRE AS CONFIGURAÇÕES DE EQUILÍBRIO}

Considere-se a barra com carregamento centrado da fig. 5.1 constituída por um material elástico ideal, com deformações diretamente proporcionais às tensões (fig. 5.2a).

Aumentando-se progressivamente a força $\mathbf{N}$, enquanto esta estiver abaixo da força crítica $\left(\mathrm{N}_{\text {crit }}\right)$ de flambagem (ver seção 3.5), os deslocamentos transversais (y) da barra serão nulos (trecho la da fig. 5.3). Quando $\mathbf{N}_{\text {crit1 }}$ é atingido, chega-se ao ponto de bifurcação do equilíbrio. Para valores superiores são possíveis duas formas de equilíbrio: uma reta e instável (trecho lc da fig. 5.3) e a forma curva estável (trecho 1 b da fig. 5.3). 


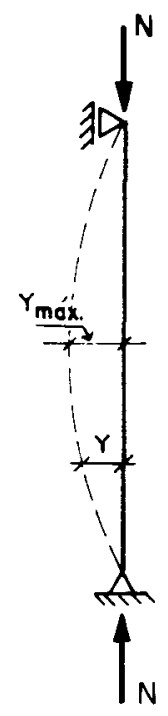

Fig. 5.1 - Barra com força axial centrada.

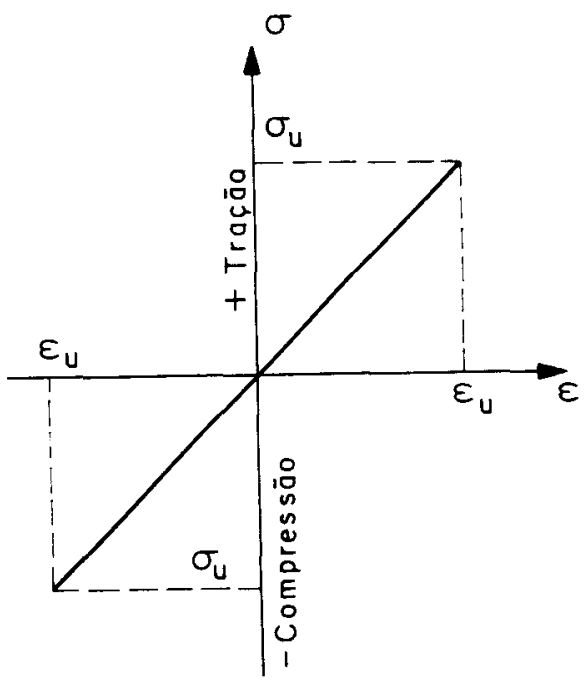

(a)

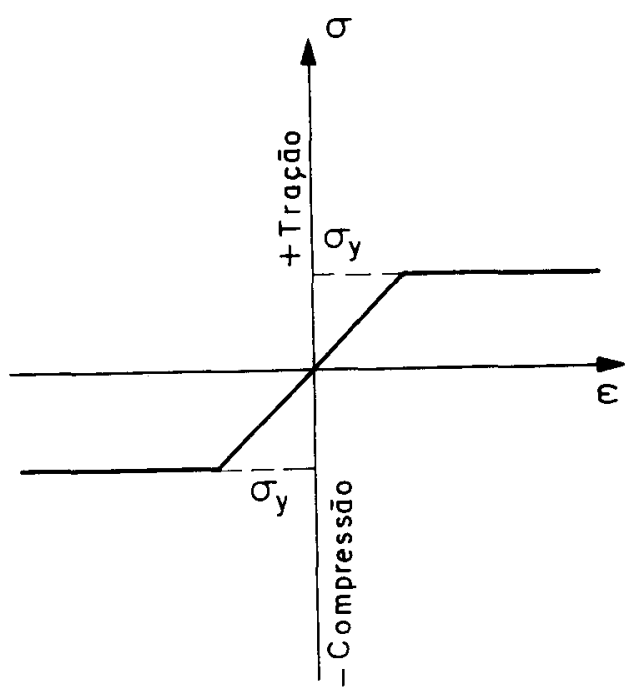

(b)

Fig. $5.2-$ Diagrama $\sigma-\varepsilon$.

Para o caso de compressão excêntrica (fig. 5.4), ainda sendo a barra constituída por material elástico ideal, para cada valor da força $\mathbf{N}$ corresponderá uma deformação $\mathbf{Y}$ (curva 2 da fig. 5.3). A força $\mathbf{N}$ pode ser aumentada até que seja atingido, em uma das bordas das seções transversais da barra, o valor máximoda tensão que o material suporta (expressão 3.13), sendo $M_{\text {ext }}=N .(e+Y)$. 


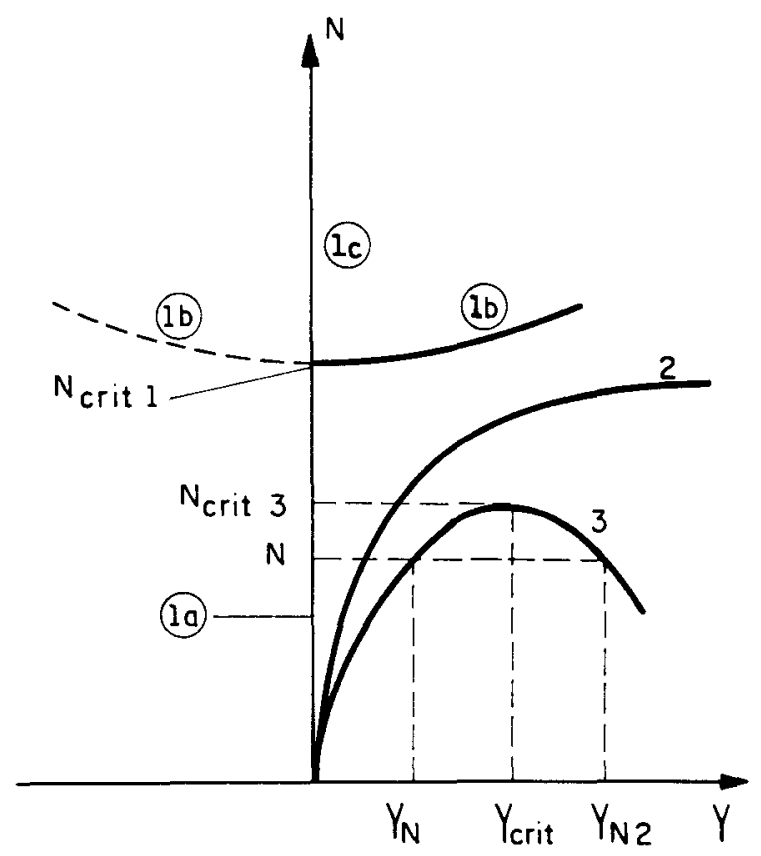

Fig. 5.3 - Curvas força-deformação.

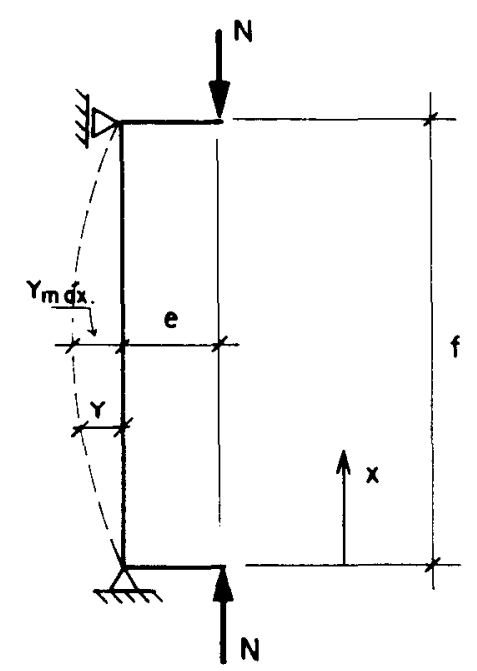

Fig. 5.4 - Barra com força axial excêntrica.

Caso o material tenha um comportamento elastoplástico ideal (fig. 5.2b), após ter sido atingido o limite de escoamento, o comportamento resistente da seção sofre modificações. A capacidade resistente da peça é esgotada quando $\mathbf{N}$ atinge $\mathbf{o}$ valor $\mathbf{N}_{\text {crit3 }}$; a plastificação da seção transversal é avançada e, para pequenas variações da força $\mathbf{N}$, o momento interno $\left(\mathrm{M}_{\text {int }}\right)$ cresce menos que o momento externo $\left(\mathrm{M}_{\mathrm{ext}}\right)$. Para $\mathbf{N}>\mathbf{N}_{\text {crit3, a barra atinge a ruína. Para aumentos da deformação correspondente à }}$ causada por $\mathbf{N}_{\text {errits }}$, o equilíbrio só será possível quando a força $\mathbf{N}$ for reduzida simultaneamente. $\mathrm{O}$ ramo descendente da curva caracteriza o estado de equilíbrio 
instável; pequenas perturbações podem conduzir ao colapso da barra (LEONHARDT, 1977).

A expressão 4.4 fornece o valor da curvatura aproximada da linha elástica, caracterizada pelo eixo deformado da barra. Em princípio, para forças superiores à força crítica de flambagem, a determinação das flechas $(Y)$ da barra exige o emprego da equação diferencial da linha elástica. Estudos mais detalhados sobre o emprego da equação exata (expressão 4.3) e da simplificada (expressão 4.4) são encontrados em FUSCO (1986) e PAULA (1988).

Para os materiais elásticos lineares, são válidas as expressões da Resistência dos Materiais (capitulo 4), onde as curvaturas das seções são funções dos momentos internos resistentes (expressão 4.2). Para materiais com comportamento elástico não linear ou elastoplástico, não há linearidade entre tensões e deformações, caso do concreto (fig. 4.4 e 4.5) e dos aços (fig. 4.7 e 4.8) utilizados em concreto armado.

Considerando-se uma seção qualquer de uma peça constituída de material elástico não-linear sujeita à flexão composta, com as deformações ocorridas e as tensões provenientes destas deformações (fig. 5.5), a obtenção do momento interno $\left(M_{\text {int }}\right)$ da seção é feita pela integração, ao longo da altura, dos momentos provenientes das tensões relativas às deformações.

$$
M_{\text {int }}=\int_{-h / 2}^{h / 2} \sigma \cdot y \cdot d A
$$

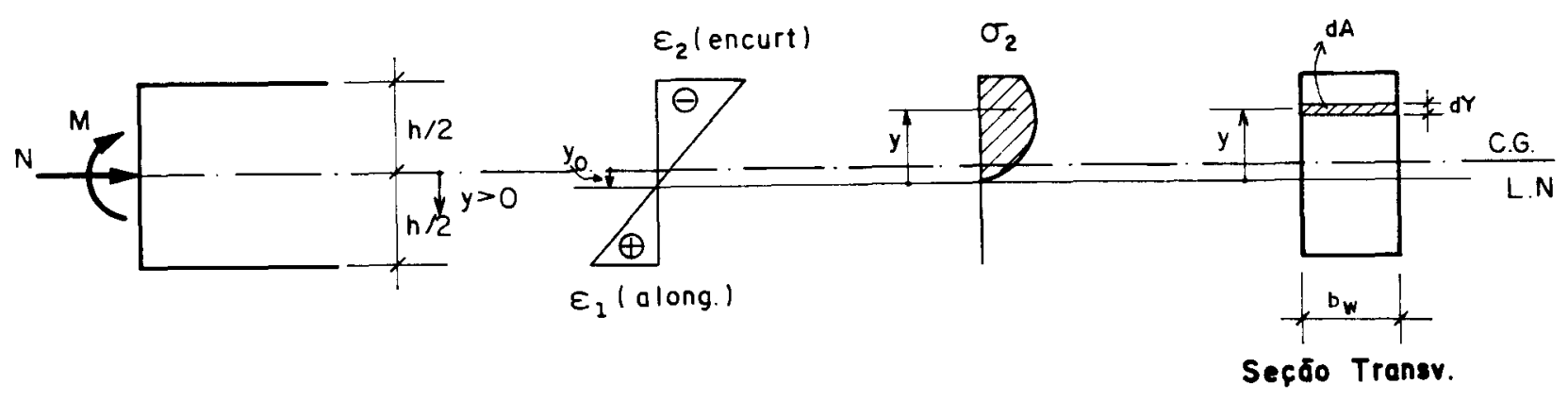

Fig. 5.5 - Deformações e tensões em uma seção, material elástico não linear.

O resultado da integral da expressão 5.1 difere do produto $(1 / r)$. E . I. A curvatura da seção pode ser obtida em função das deformações: 
$\frac{1}{r}=\frac{-\varepsilon_{2}}{\frac{h}{2}+y_{0}}=\frac{\varepsilon_{1}}{\frac{h}{2}-y_{0}}$

Os momentos externos solicitantes são obtidos pela expressão:

$$
\mathrm{M}_{\mathrm{ext}}=\mathrm{N} \cdot(\mathrm{e}+\mathrm{Y})
$$

Onde Y é o deslocamento do eixo da barra sob a ação das forças externas.

Para a manutenção do equilíbrio da barra, a igualdade entre os momentos externos e os momentos internos deve ser satisfeita em cada seção da barra.

$$
\mathbf{M}_{\text {int }}=\mathbf{M}_{\mathrm{ext}}
$$

Adotando-se uma linha elástica senoidal para a barra da fig. 5.4 constituída por material elástico não-linear, tem-se:

$$
\mathrm{Y}=\mathrm{Y}_{\text {max. }} \cdot \operatorname{sen}\left(\frac{\pi}{l}\right) \mathrm{x}
$$

A adoção da linha elástica senoidal e o emprego da equação simplificada da curvatura levam a uma relação linear do momento externo $\left(\mathrm{M}_{\mathrm{ext}}\right)$ em função da curvatura (FUSCO, 1986). Derivando duas vezes a expressão 5.5 em relação a $\mathbf{x}$, tem-se:

$$
\begin{aligned}
& \frac{\mathrm{d}^{2} \mathrm{Y}}{\mathrm{dx}^{2}}=-\mathrm{Y}_{\max }\left(\frac{\pi}{l}\right)^{2} \cdot \operatorname{sen}\left(\frac{\pi}{l} \mathrm{x}\right) \\
& \frac{1}{\mathrm{r}} \cong-\left(\frac{\pi}{l}\right)^{2} \cdot \mathrm{Y}_{\max }
\end{aligned}
$$

Em valor absoluto: 
$\mathrm{Y}_{\max }=\left(\frac{l}{\pi}\right)^{2} \cdot \frac{1}{\mathrm{r}}$

Com base nas expressões 5.3 e 5.7 , tem-se:

$$
\mathrm{M}_{\mathrm{ext}}=\mathrm{N} \cdot \mathrm{e}+\mathrm{N} \cdot\left(\frac{l}{\pi}\right)^{2} \cdot \frac{1}{\mathrm{r}}
$$

Colocando-se em um mesmo gráfico os momentos internos (função nãolinear da curvatura) e externos em função das curvaturas da seção (fig. 5.6), com valores crescentes para a força $\mathbf{N}$, verifica-se que para valores de $\mathbf{N}$ inferiores à força crítica há sempre configuração fletida de equilíbrio estável para a barra, uma vez que a reta dos momentos externos encontra a curva dos momentos internos, satisfazendo a condição de equilíbrio da expressão 5.4. Quando $\mathbf{N}$ alcança o valor $\mathbf{N}_{\text {crit }}$, a reta dos momentos externos tangencia a curva dos momentos internos, caracterizando o equilíbrio instável. Para valores maiores que $\mathbf{N}_{\text {crit }}$, não há equilíbrio possível.

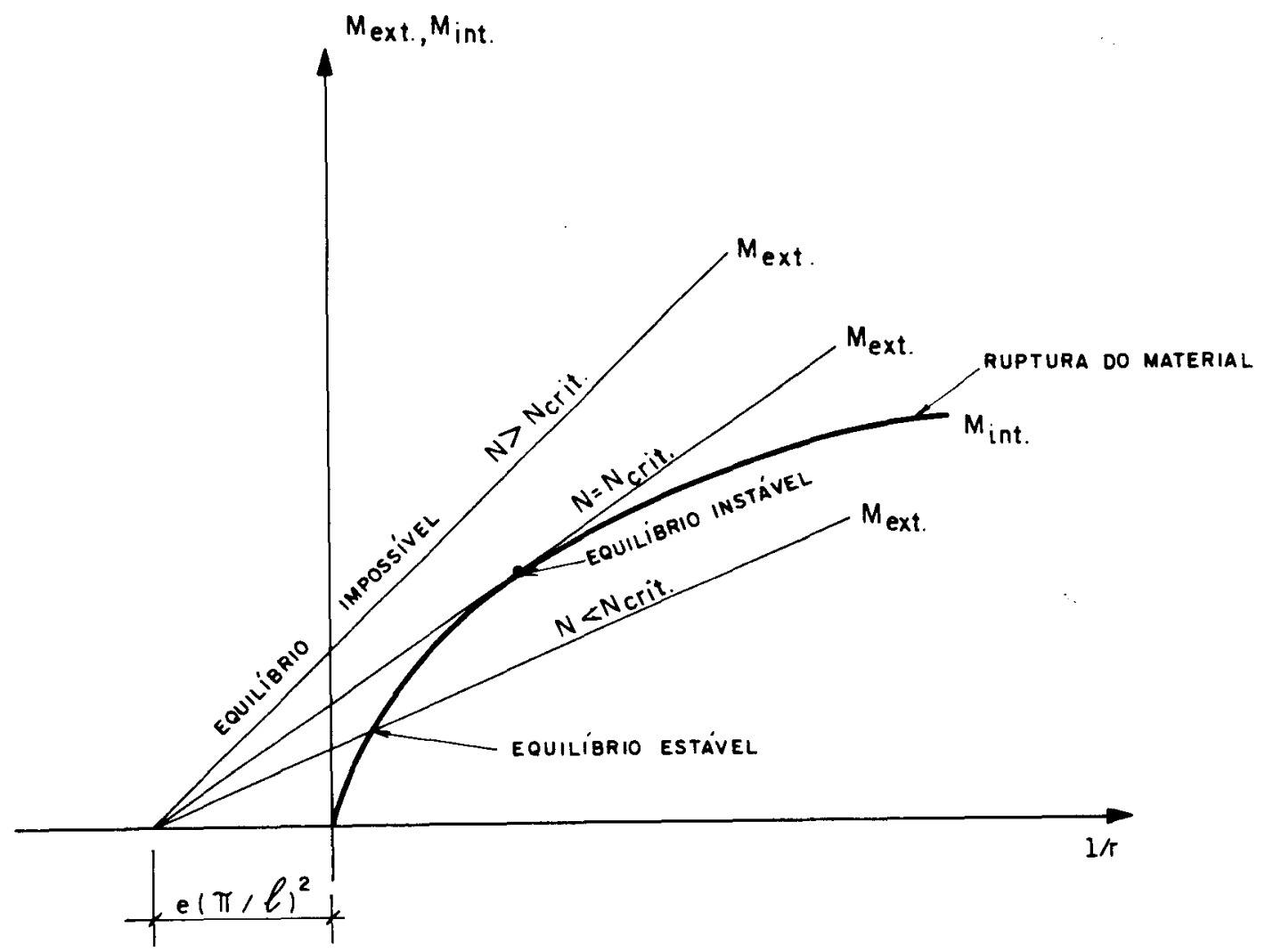

Fig. 5.6 - Instabilidade na flexo-compressão, material elástico não-linear. 


\subsection{CÁlCULO DA FORÇA CRÍTICA: MÉTODO GERAL}

Tem-se, para pilares de seção variável, dois métodos para a verificação da estabilidade da configuração de equilíbrio: o método geral e o método do equilíbrio. Em ambos são consideradas tanto a não-linearidade geométrica do elemento quanto a não-linearidade fisica do material do qual é constituído.

O método geral é aplicável a qualquer tipo de estrutura com qualquer tipo de carregamento. Deve-se determinar um diagrama força-deslocamento, considerandose um parâmetro $(\alpha)$ que represente o carregamento aplicado e um ponto de referência, normalmente o de maiores deslocamentos, para a aferição da estabilidade da configuração de equilíbrio (fig. 5.7a). Um ponto de máximo relativo no diagrama caracteriza a instabilidade da peça, determinando a força crítica (fig. 5.7b).
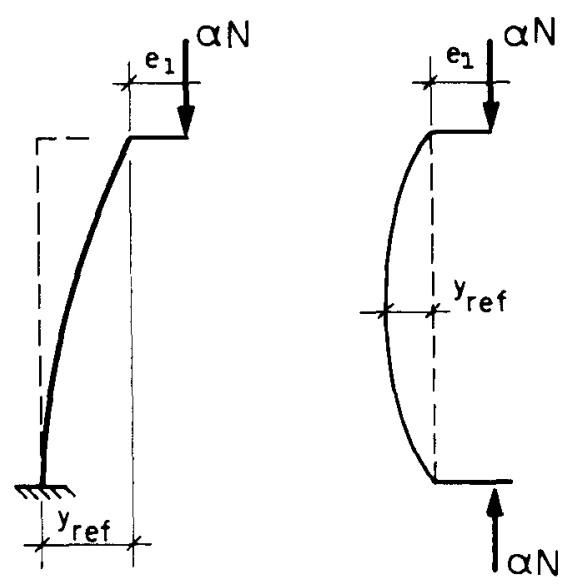

(0)

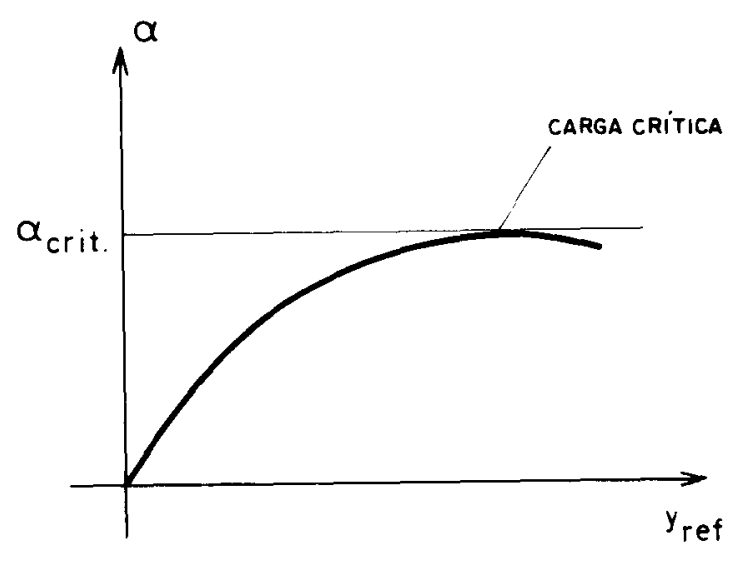

(b)

Fig. 5.7 - Gráfico carregamento proporcional - desiocamento.

Pode-se determinar a instabilidade da peça em função da intensidade da força aplicada (processo do carregamento progressivo proporcional) ou da excentricidade de aplicação da força (processo das excentricidades progressivas).

\subsubsection{PROCESSO DO CARREGAMENTO PROGRESSIVO PROPORCIONAL}

É um processo "exato" que deve ser empregado em peças de grande esbeltez ou de seção transversal variável ao longo do comprimento (FUSCO, 1986). Para a determinação da força crítica, adota-se a seqüência a seguir: 
a) $\mathbf{O}$ carregamento é aplicado por incrementos progressivos $\Delta \mathbf{N}_{\mathbf{i}}$, partindo de zero e aumentando todas as forças proporcionalmente ao mesmo coeficiente $\alpha$.

b) Para cada etapa de carregamento $\left(\alpha_{\mathrm{i}} \mathrm{N}\right)$, calcula-se o deslocamento $\left(\mathrm{y}_{\text {ref }}\right)$ da seção de referência. Além dos efeitos de $1^{\underline{a}}$ ordem, devidos ao carregamento $\alpha \mathrm{N}_{\mathrm{i}}$, devem ser considerados os momentos de $2^{\mathrm{a}}$ ordem provenientes das deformações causadas pelo carregamento da etapa anterior (fig. 5.8).

c) O carregamento crítico é obtido por $\alpha_{c r i t} \mathrm{~N}$, para o qual tende assintoticamente o diagrama força-deslocamento (fig. 5.9).
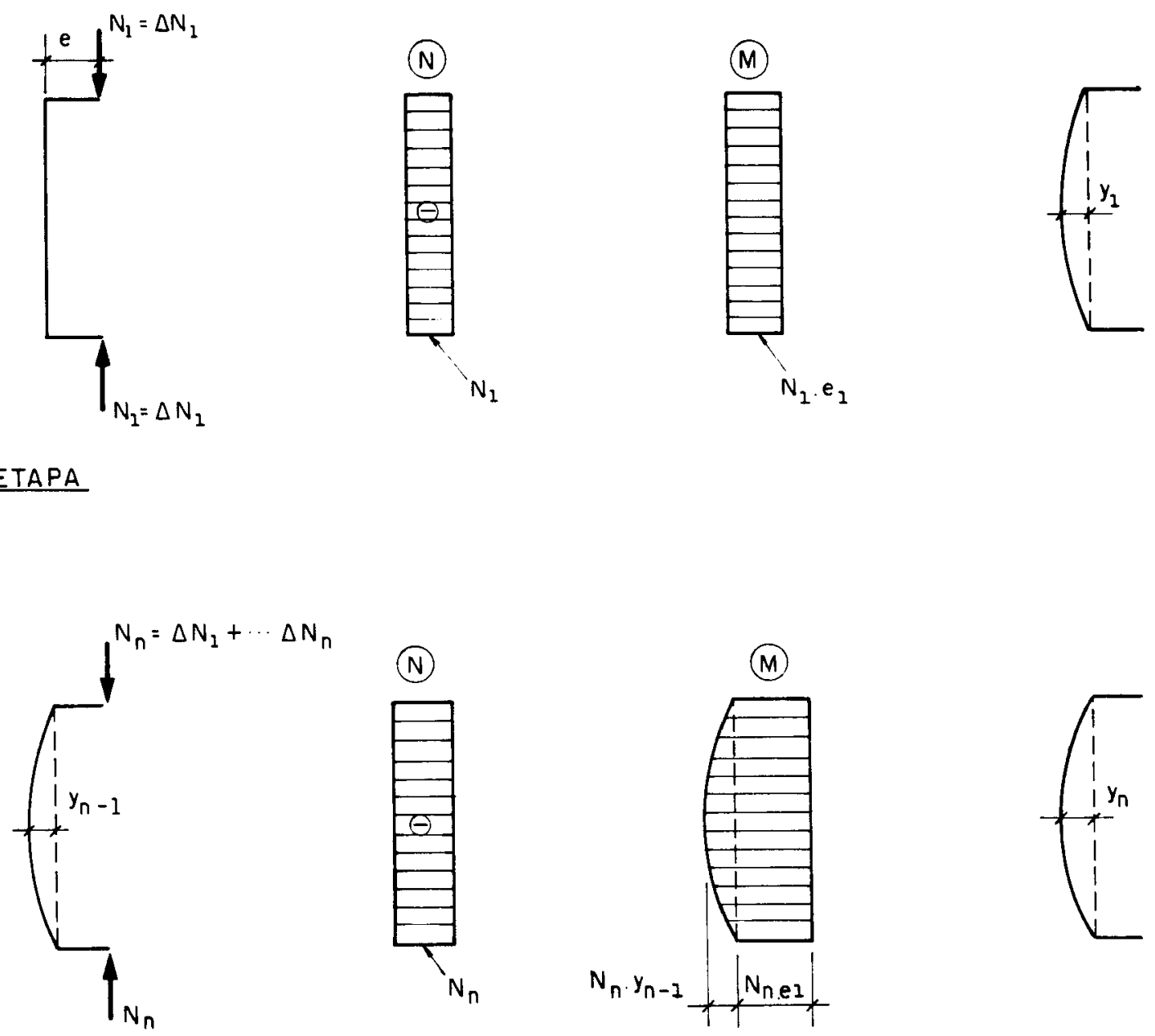

Fig. 5.8 - Etapas de carregamento.

A precisão dos resultados depende da grandeza dos incrementos de força aplicados. As estruturas hiperestáticas são resolvidas a cada etapa de carregamento, considerando-se a não-linearidade geométrica do sistema e a não-linearidade física 
do material. Os métodos para a obtenção dos valores dos deslocamentos serão tratados no capítulo 7 .

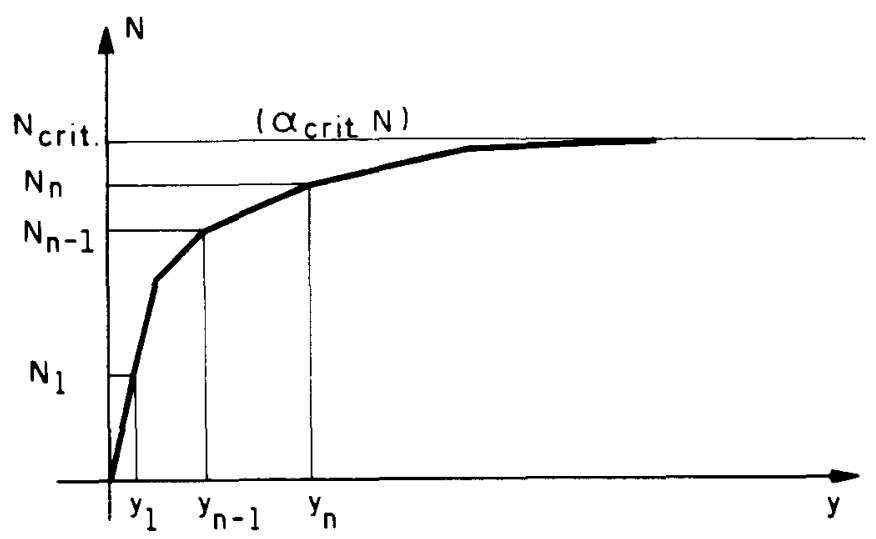

Fig. 5.9 - Diagrama força-deslocamento.

\subsubsection{PROCESSO DAS EXCENTRICIDADES PROGRESSIVAS}

A força normal é mantida constante, impondo-se a variação à excentricidade de $1^{\underline{a}}$ ordem $\left(e_{1}\right)$. Na primeira etapa do processo, aplica-se a excentricidade $e_{1,1}=\Delta e_{1}$

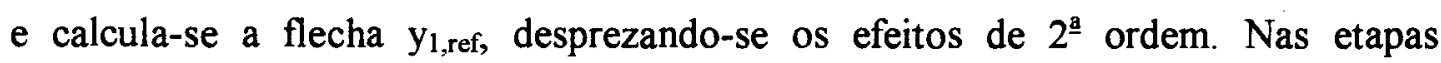
seguintes, considera-se a deformação causada pela excentricidade da etapa anterior (fig. 5.10).

$\mathrm{O}$ valor crítico da excentricidade é obtido com o valor assintótico $\mathrm{e}_{1, \text { crit, }}$ indicado na fig. 5.11. Conhecendo-se o diagrama excentricidade-deslocamento, pode ser construído o diagrama momento total - deslocamento (fig. 5.12). Pela subtração do momento de $2^{\mathrm{a}}$. ordem $\left(\mathrm{M}_{2}=\mathrm{F}\right.$.y), pode ser obtido o valor crítico do momento de $1^{\text {a }}$ ordem $\left(\mathrm{M}_{1, \text { crit }}\right)$. 

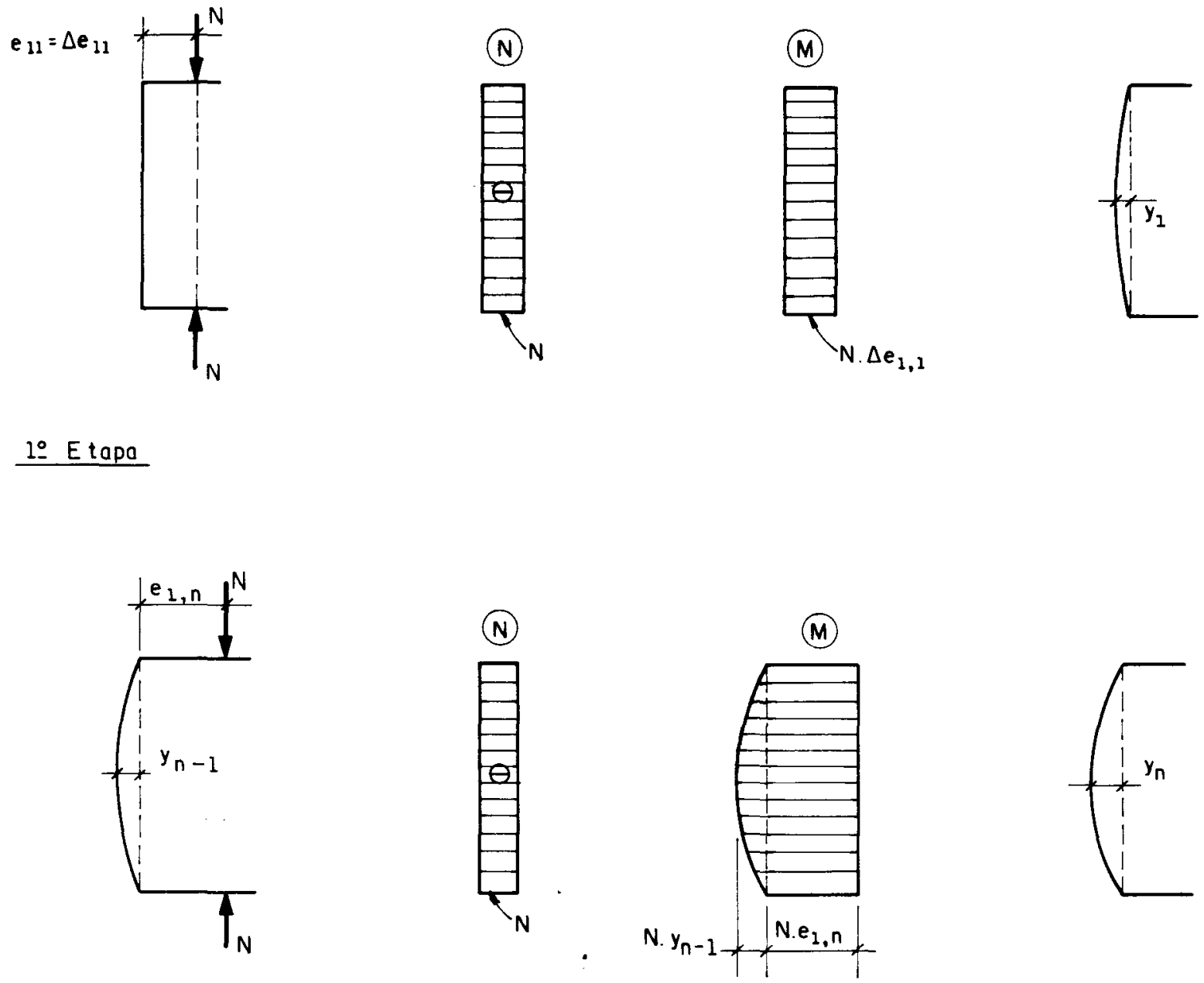

n․ Etapo

Fig. 5.10 - Etapas de cálculo: processo das excentricidades progressivas.

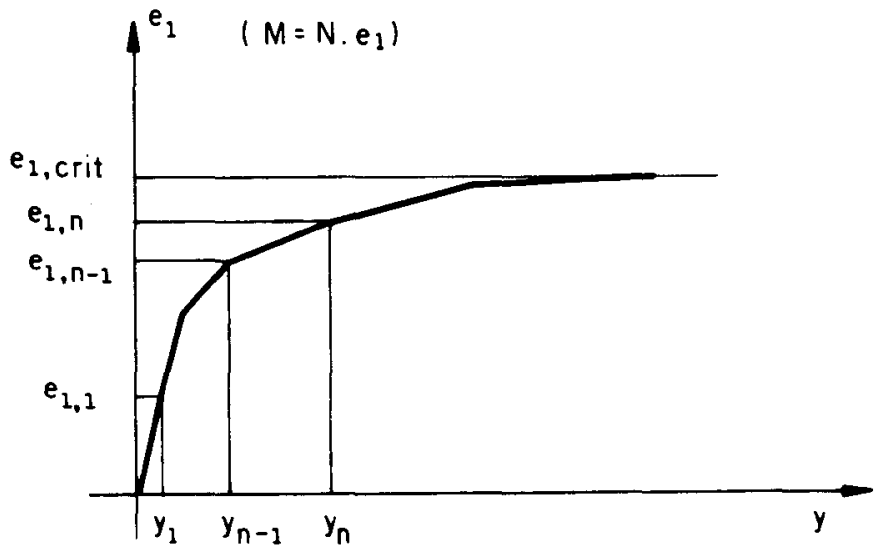

Fig. 5.11 - Determinação do valor de $e_{1, \text { crit }}$. 


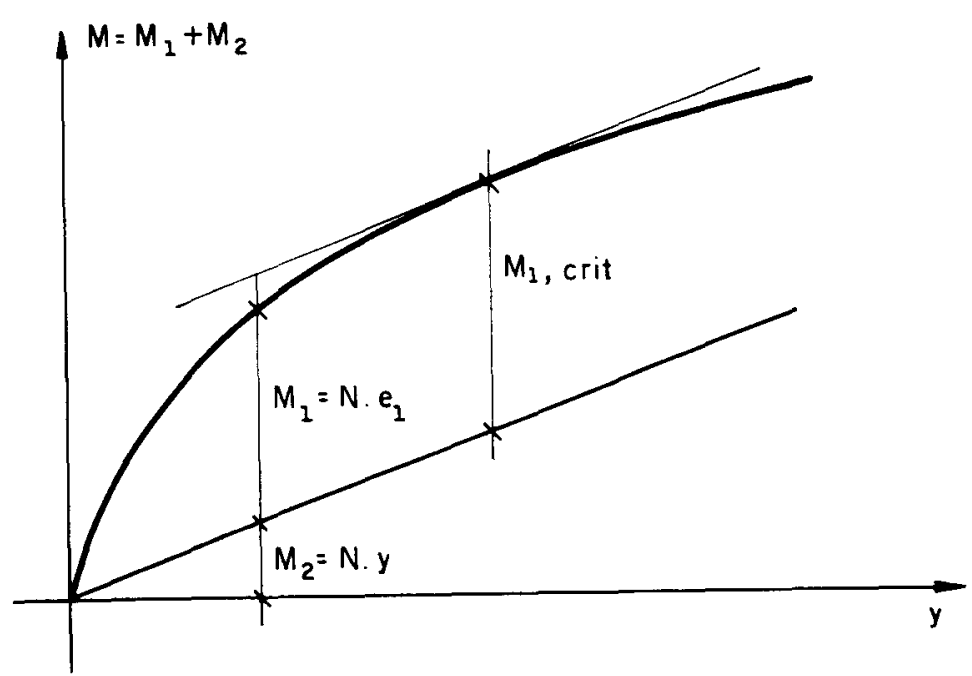

Fig. 5.12 - Determinação do valor de $\mathbf{M}_{1, \text { crit }}$.

\subsection{CÁlCULO DA FORÇA CRÍTICA: MÉTODO DO EQUILÍBRIO}

Este método, com o processo dos deslocamentos de referência, consiste em garantir a segurança contra o estado limite de instabilidade, através da verificação de que, sob a ação da força de cálculo $\mathbf{N}_{\mathbf{d}}$, ou da excentricidade de cálculo $\mathbf{e}_{\mathbf{1 d}}$, a flecha

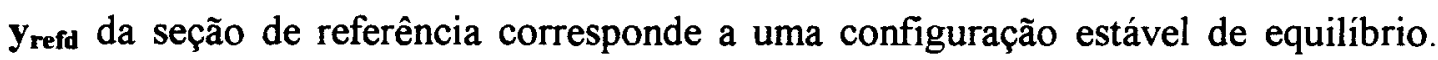
Procede-se de maneira análoga à exposta na seção 5.2.1, adotando-se a seqüência a seguir (fig. 5.13):

a) Cálculo do deslocamento da seção de referência considerando apenas os efeitos de $1^{\text {a }}$. ordem.

b) Cálculo do deslocamento da seção de referência considerando a deformação da etapa anterior.

c) Repetição do passo b até a convergência dos valores dos deslocamentos da seção de referência, o que comprova a estabilidade da configuração de equilíbrio.

Pelo fato da seqüência ser construída a partir da flecha decorrente dos efeitos de $1^{\underline{a}}$ ordem, quando a série for convergente $o$ equilíbrio será estável, correspondendo necessariamente ao ramo ascendente da curva força-deslocamento (FUSCO, 1986) 

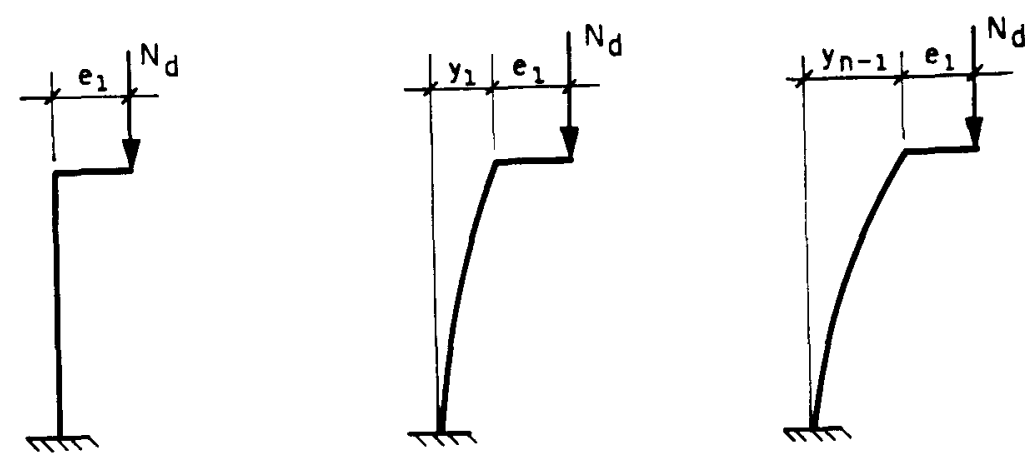

Fig. 5.13 Etapas do processo do deslocamento de referência.

A convergência é constatada numericamente. Quando ocorre, sabe-se que o ponto $\mathbf{N}_{\mathbf{d}}$ está abaixo do ponto $\mathbf{N}_{\mathbf{c r t t}}$ e a estrutura tem segurança abundante; porém não fica quantificado o excesso existente. Com isso, calcula-se apenas um ponto do diagrama força-deslocamento (fig. 5.14).

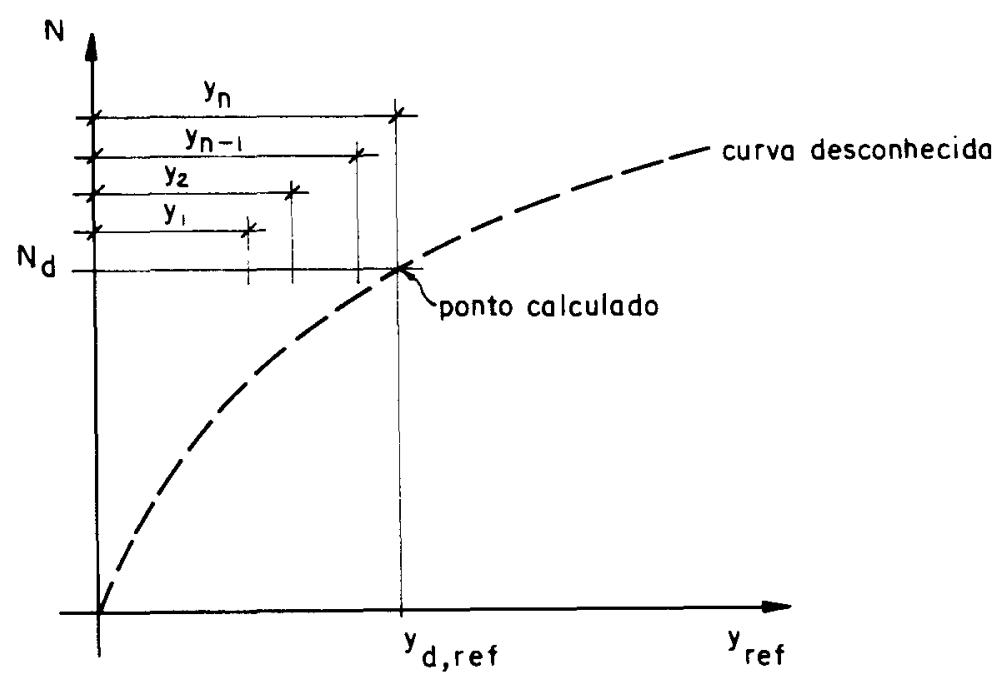

Fig. 5.14 - Deslocamentos calculados para $\mathrm{N}_{\mathrm{d}}$. 


\section{DIAGRAMAS MOMENTO FLETOR - FORÇA NORMAL - CURVATURA}

Estes diagramas são utilizados para o dimensionamento e estudo da estabilidade de elementos de concreto armado, considerando-se a não-linearidade fisica dos materiais e a não-linearidade geométrica do elemento estrutural.

Apresentam-se as expressões destinadas à obtenção do momento fletor absorvido por seções retangulares e circulares de concreto armado (cheias e vazadas), em função da curvatura proveniente da flexão da barra sob a ação de uma força normal. Estas expressões foram desenvolvidas com base nos diagramas tensãodeformação dos materiais constituintes dos elementos (seções 4.3 e 4.4), recomendados pela Norma Brasileira NBR-6118/1978 e pelo Código Modelo do Comité Euro-Internacional du Béton CEB-1990, empregando-se para seu desenvolvimento o software DERIVE 2.01 (1988-1990).

Segue-se aqui a metodologia utilizada por PAULA (1988), estudo realizado para seções retangulares cheias com diagrama parábola-retângulo para as tensões e deformações no concreto, recomendação da já citada NBR-6118/1978.

Seções submetidas à flexão oblíqua não foram objeto de estudo deste trabalho, podendo-se encontrar considerações relativas a este tipo de flexão em FUSCO (1986)

\subsection{CURVATURA E DEFORMAÇÃO DAS SEÇÕES}

Considerando-se a seção da figura 6.1a, sujeita à flexão normal composta, e as deformações genéricas ocorridas nesta seção (sendo válida a hipótese de Bernoulli), segundo o eixo EE' (fig. 6.2b), onde: 
$\varepsilon_{\mathrm{c} 2} \Rightarrow$ deformação específica do concreto na borda mais comprimida (encurtamento).

$\varepsilon_{3 / 7} \Rightarrow$ deformação específica no concreto distante de $(3 / 7) \mathrm{h}$ da borda mais comprimida da seção.

$\varepsilon \Rightarrow$ deformação específica genérica do concreto distante y do eixo do centro de gravidade (LCG) da seção.

$\varepsilon_{\mathrm{s}} \Rightarrow$ deformação específica do aço da armadura tracionada.

$\mathrm{x} \Rightarrow$ distância da borda mais comprimida à linha neutra (LN).

$y_{0} \Rightarrow$ distância da linha neutra ao eixo do centro de gravidade da seção.

$\mathrm{d}^{\prime} \Rightarrow$ distância do centro de gravidade da armadura à borda mais próxima da seção.

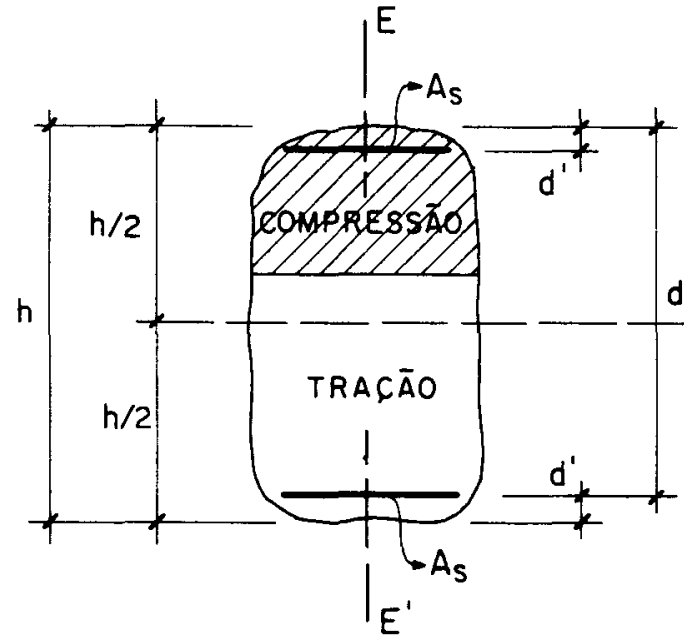

a)

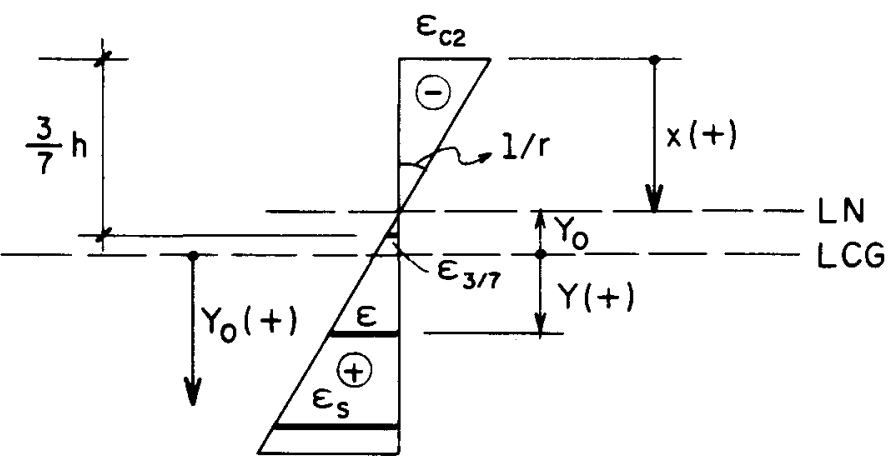

b)

Fig. 6.1 - Deformações em uma seção de concreto armado.

Com base na figura $6.1 \mathrm{~b}$, pode-se expressar a deformação específica genérica do concreto $(\varepsilon)$ em função de $\varepsilon_{c 2}, \varepsilon_{\mathrm{s}}$ e $\varepsilon_{3 / 7}$ :

$$
\begin{aligned}
& \varepsilon=\frac{-\varepsilon_{c 2}}{x}\left(y-y_{0}\right) \\
& \varepsilon=\frac{\varepsilon_{s}}{\left(h-d^{\prime}-x\right)}\left(y-y_{0}\right)
\end{aligned}
$$




$$
\varepsilon=\frac{\varepsilon_{3 / 7}}{(3 / 7) h-x}\left(y-y_{0}\right)
$$

As deformações específicas do concreto na borda mais comprida e a $3 / 7$ da altura, bem como a deformação específica da armadura tracionada, são relacionadas à curvatura da seção pelas expressões 6.4 a 6.6 , onde $\operatorname{tg}(1 / \mathrm{r}) \cong 1 / \mathrm{r}$ :

$$
\begin{aligned}
& \varepsilon_{\mathrm{c} 2}=-\frac{1}{\mathrm{r}} \mathrm{x} \\
& \varepsilon_{\mathrm{s}}=\frac{1}{\mathrm{r}}\left(\mathrm{h}-\mathrm{d}^{\prime}-\mathrm{x}\right) \\
& \varepsilon_{3 / 7}=\frac{1}{\mathrm{r}}\left(\frac{3}{7} \mathrm{~h}-\mathrm{x}\right)
\end{aligned}
$$

Pela substituição das expressões 6.4 a 6.6 nas expressões 6.1 a 6.3 , respectivamente, e pela figura $6.1 \mathrm{~b}$, tem-se:

$$
\varepsilon=\frac{1}{r}\left(y-y_{0}\right)
$$

que é a deformação específica do concreto em um ponto qualquer da seção.

É usual expressar a posição da linha neutra (x), de um ponto genérico da seção (y) e dos demais parâmetros apresentados em termos adimensionais, ou seja, divididos pela altura (h) da seção. Assim, tem-se:

$$
\begin{aligned}
& \beta_{\mathrm{x}}=\mathrm{x} / \mathrm{h} \\
& \beta \mathrm{y}=\mathrm{y} / \mathrm{h} \\
& \delta=\mathrm{d}^{\prime} / \mathrm{h}
\end{aligned}
$$

Da figura 6.1b e da expressão 6.7 , tem-se: 


$$
\varepsilon=\frac{1}{r}(y-x+h / 2)
$$

em termos adimensionais:

$$
\varepsilon=\frac{h}{r}\left(\beta_{y}-\beta_{x}+1 / 2\right)
$$

A expressão 6.12 permite o cálculo da deformação específica do concreto ou do aço em um ponto genérico da seção - distante y do eixo do centro de gravidade em função da curvatura e da posição da linha neutra $\left(\beta_{\mathrm{x}}\right)$.

Para a caracterizações das deformações genéricas ocorridas em uma seção de concreto armado (fig. 6.1b), é necessária a adoção da curvatura da seção e da profundidade da linha neutra - definida na forma adimensional por $\beta_{\mathrm{x}}$ (expressão 6.8). De acordo com a convenção de sinais apresentada (fig. 6.1b), teoricamente $\beta_{x}$ pode variar de $-\infty$ (tração centrada) a +o (compressão centrada). Os valores deste parâmetro adimensional e da curvatura da seção devem respeitar os limites últimos de deformações do concreto e do aço (seção 4.5).

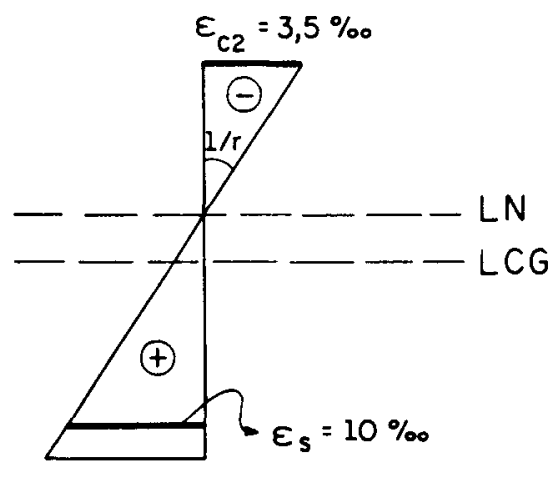

Fig. 6.2 - Deformações máximas convencionais para uma seção de concreto armado.

Das figuras 6.1 e 6.2 pode-se obter a expressão que fornece o valor da máxima curvatura, respeitando-se os limites de deformação:

$$
\frac{1}{r}=\frac{0,0135}{d}
$$


ou, na forma adimensional:

$$
\frac{\mathrm{h}}{\mathrm{r}}=\frac{0,0135}{(1-\delta)}
$$

Para a definição do intervalo de variação de $\beta_{\mathrm{x}}$, dentro dos limites últimos de deformação e da convenção adotada para a variação de $\beta_{\mathrm{x}}$ segundo o eixo $E E^{\prime}$ da figura 6.3 , pode-se ter três situações:
a) $\beta_{\mathrm{x}} \leq 0 \quad \Rightarrow$ seção totalmente tracionada;
b) $0<\beta_{x}<1 \Rightarrow$ seção parcialmente comprimida;
c) $\beta_{x} \geq 1 \quad \Rightarrow$ seção totalmente comprimida.

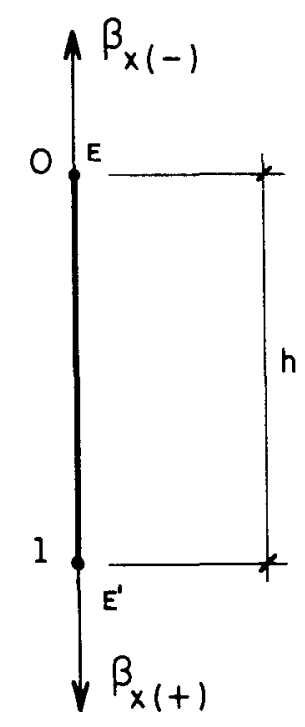

Fig. 6.3 - Convenção para a variação do parâmetro adimensional $\beta_{x}$.

Para $\beta_{\mathrm{x}} \leq 0$, a seção encontra-se no domínio 1 , o concreto é suposto inteiramente fissurado e a profundidade da linha neutra $\left(\beta_{\mathrm{x}}\right)$ é determinada pela deformação de tração do aço $\left(\varepsilon_{\mathrm{s}}=0,01\right)$, definindo o limite inferior $\left(\beta_{\mathrm{xi}}\right)$ do intervalo de variação da linha neutra (fig. 6.4).

A posição dimensional da armadura mais tracionada $(\beta y)$, em relação ao eixo do centro de gravidade, é obtida por: 


$$
\begin{aligned}
& \beta y=\left[(h / 2)-d^{\prime}\right] / h \\
& \beta y=1 / 2-\delta
\end{aligned}
$$

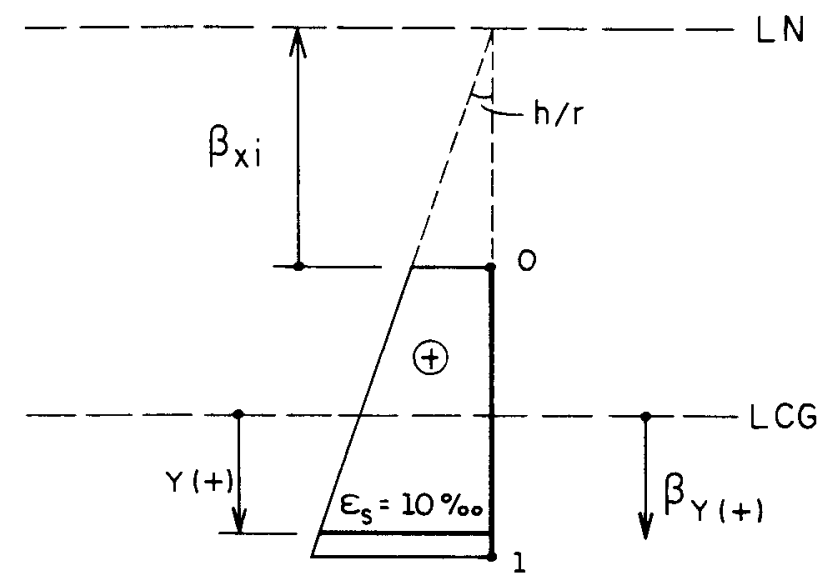

Fig. 6.4 - Deformação em uma seção de concreto inteiramente tracionada.

Substituindo-se a expressão 6.15 na expressão 6.12 e limitando a deformação específica do aço em 0,01 , para que não seja ultrapassado o limite último de deformação, tem-se:

$$
\varepsilon=\frac{\mathrm{h}}{\mathrm{r}}\left(1-\delta-\beta_{\mathrm{x}}\right) \leq 0,01
$$

onde $\beta_{\mathrm{x}}=\beta_{\mathrm{x} i}$; isolando-se $\beta_{\mathrm{xi}}$ :

$$
\beta_{\mathrm{xi}} \geq-\frac{0,01}{(\mathrm{~h} / \mathrm{r})}+1-\delta
$$

Para $0<\beta_{\mathrm{x}}<1$, a seção encontra-se nos domínios 2, 3, 4 ou 4a, o limite inferior da variação da linha neutra $\left(\beta_{\mathrm{xi}}\right)$ é definido pela expressão 6.16 e o limite superior $\left(\beta_{\mathrm{xs}}\right)$ pela deformação do concreto na borda mais comprimida (fig. 6.5), que é limitada pelo valor $-0,0035$ (encurtamento). 


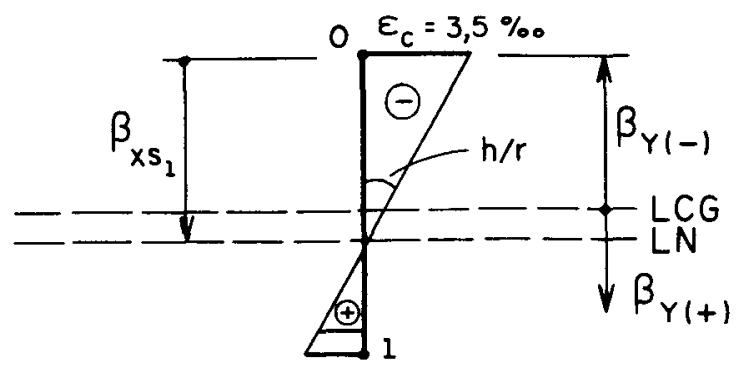

Fig. 6.5 - Deformação em uma seção de concreto parcialmente comprimida.

A posição da borda mais comprimida da seção em relação ao eixo do centro de gravidade (LCG) é obtida por:

$$
\begin{aligned}
& \beta_{y}=\frac{(-\mathrm{h} / 2)}{h} \\
& \beta_{y}=-1 / 2
\end{aligned}
$$

Substituindo-se 6.17 em 6.12 e limitando-se a deformação específica do concreto em $-0,0035$, tem-se:

$$
\varepsilon=\frac{h}{r}\left(-\beta_{x}\right) \geq-0,0035
$$

onde $\beta_{\mathrm{x}}=\beta_{\mathrm{xsl}}$, isolando-se $\beta_{\mathrm{xsl}}$ :

$$
\beta_{\mathrm{xs} 1} \leq \frac{0,0035}{(\mathrm{~h} / \mathrm{r})}
$$

Para $\beta_{x} \geq 1$, a seção encontra-se no domínio 5 , determinando um segundo valor para o parâmetro superior de variação da linha neutra, caracterizado pela deformação $-0,002$ a (3/7)h da borda mais comprimida da seção (fig. 6.6).

A posição do ponto onde $\varepsilon_{3 / 7}=-0,002$ é definido por:

$$
\beta_{y}=\left[-\frac{h}{2}-\left(-\frac{3}{7} h\right)\right] / h
$$


$\beta_{\mathrm{y}}=-1 / 14$

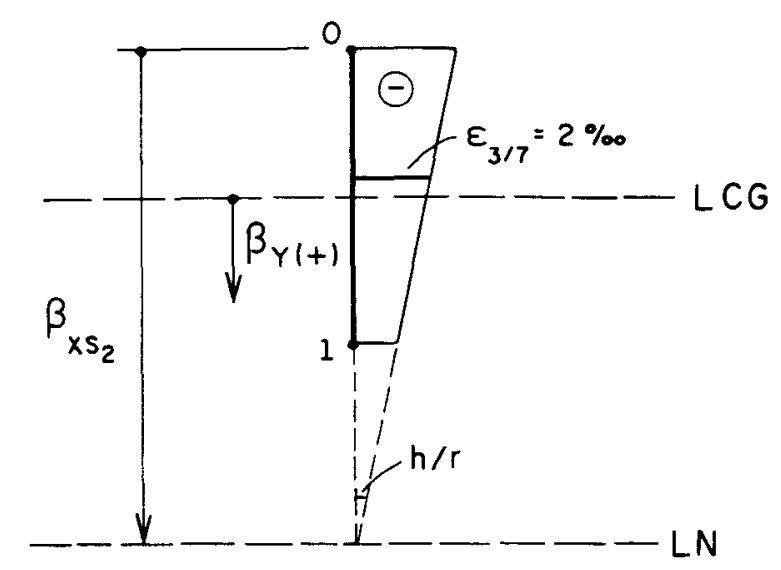

Fig. 6.6 - Deformação em uma seção de concreto inteiramente comprimida.

Substituindo-se 6.19 em 6.12 e limitando a deformação especifica do concreto em - 0,002, tem-se:

$$
\varepsilon=\frac{\mathrm{h}}{\mathrm{r}}\left(\frac{3}{7}-\beta_{\mathrm{x}}\right) \geq-0,002
$$

onde $\beta_{\mathrm{x}}=\beta_{\mathrm{xs} 2}$

Isolando-se $\beta_{\mathrm{xs} 2}$, tem-se:

$$
\beta_{\mathrm{xs} 2} \leq \frac{0,002}{(\mathrm{~h} / \mathrm{r})}+\frac{3}{7}
$$


A escolha do parâmetro superior de variação da linha neutra $\left(\beta_{\mathrm{xs}}\right)$ depende de qual dos valores, obtidos pelas expressões 6.18 e 6.20, é atingido primeiro (menor valor absoluto).

\subsection{EQUAÇÕES DE EQUILÍBRIO}

Viu-se na seção 5.1 que a manutenção do equilíbrio da barra depende da igualdade entre os esforços externos solicitantes e os esforços internos resistentes das seções das barras. Considerando-se válida a hipótese de Bernoulli, arbitrando-se valores para a curvatura e profundidade da linha neutra da seção, obtêm-se as deformações dos pontos genéricos desta seção pela expressão 6.12. Com estas deformações e os gráficos tensão-deformação dos materiais (seções 4.3 e 4.4) determina-se a distribuição das tensões resistentes.

Os esforços internos resistentes, empregados nas relações de equilibrio, são obtidos pela integração das tensões resistentes ao longo da seção. De modo geral, as expressões que fornecem a normal resistente $\left(\mathrm{N}_{\mathrm{I}}\right)$ e o momento fletor resistente $\left(\mathrm{M}_{\mathrm{I}}\right)$ são:

$$
\begin{aligned}
& N_{\mathrm{l}}=\mathrm{R}_{\mathrm{c}}+\sum_{\mathrm{i}=1}^{\mathrm{n}} \mathrm{R}_{\mathrm{si}} \\
& \mathrm{M}_{\mathrm{I}}=\mathrm{M}_{\mathrm{c}}+\sum_{\mathrm{i}=1}^{\mathrm{n}} \mathrm{R}_{\mathrm{si}} \cdot \mathrm{Y}_{\mathrm{si}}
\end{aligned}
$$

onde:

$\mathrm{n}$ = número de barras da armadura

$\mathbf{R}_{\mathbf{c}}=$ resultante de tensões normais na região comprimida da seção de concreto

$\mathbf{M}_{\mathrm{c}}=$ resultante das tensões de compressão região comprimida da seção de concreto

$\mathrm{R}_{\mathrm{si}}=$ resultante de tensões normais nas seções das barras de aço da camada $\boldsymbol{i}$

$\mathrm{Y}_{\mathrm{si}}=$ distância das barras de aço da camada $i$ ao eixo do centro de gravidade da seção de concreto

$\mathrm{i}$ = índice relativo às posições das barras de aço. 


$$
\begin{aligned}
& \mathbf{R}_{c}=\int_{\mathbf{A}_{c}} \sigma_{c} \cdot \mathrm{dA}_{c} \\
& \mathbf{R}_{\mathrm{si}}=\mathrm{A}_{\mathrm{si}} \cdot \sigma_{\mathrm{si}} \\
& \mathbf{M}_{\mathrm{C}}=\int_{\mathrm{A}_{\mathrm{c}}} \mathrm{Y} \cdot \sigma_{\mathrm{C}} \cdot \mathrm{dA}_{\mathrm{C}}
\end{aligned}
$$

onde:

$\mathrm{A}_{\mathrm{si}}=$ área das seções das barras de aço da camada $i$

$\mathrm{A}_{\mathrm{c}}=$ área de concreto

$\sigma_{\mathrm{c}}=$ tensão normal no concreto

$\sigma_{\mathrm{si}}=$ tensão normal nas seções das barras de aço da camada $i$

y = distância do ponto de aplicação da resultante das tensões de compressão ao eixo do centro de gravidade da seção.

\subsubsection{SEÇÕES RETANGULARES CHEIAS}

Considerando-se a seção da figura 6.7a e as deformações nela ocorridas (fig. 6.7b), além das expressões 6.23 e 6.24 , pode-se escrever as expressões 6.21 e 6.22 em função da variável $y$, sendo $d A=b_{w}$. dy

$$
\begin{aligned}
& N_{I}=\int_{-h / 2}^{y_{0}} b_{w} \cdot \sigma_{c} \cdot d y+\sum_{i=1}^{n} \sigma_{s i} \cdot A_{s i} \\
& M_{I}=\int_{-h / 2}^{y_{0}} b_{w} \cdot y \cdot \sigma_{c} \cdot d y+\sum_{i=1}^{n} y \cdot \sigma_{s i} \cdot A_{s i}
\end{aligned}
$$




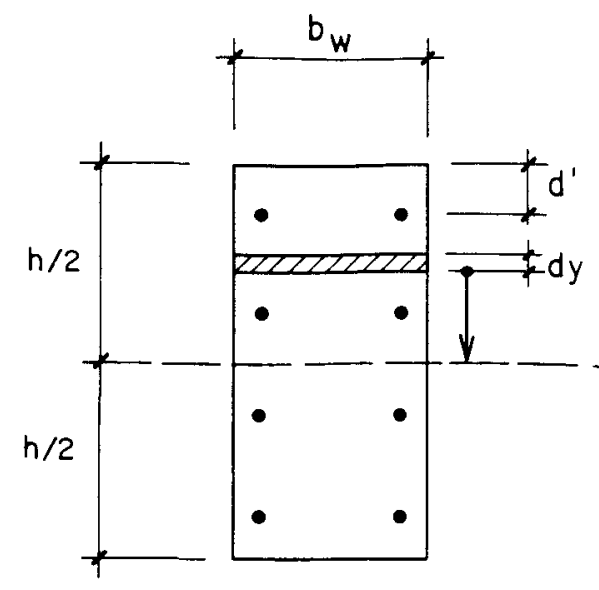

o)

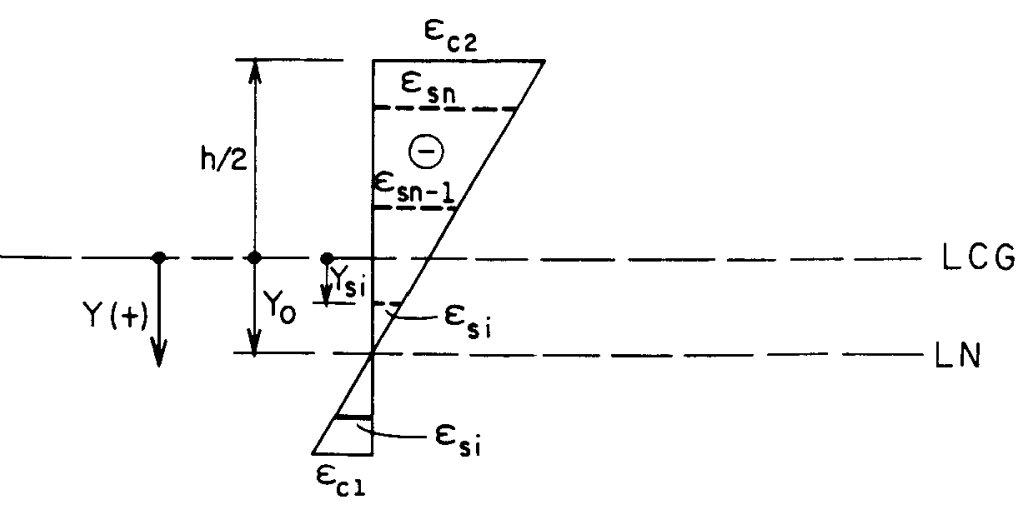

b)

Fig. 6.7 - Deformações em uma seção retangular de concreto armado.

\subsubsection{SEÇÕES RETANGULARES VAZADAS}

Considerando-se a seção da fig. 6.8a e as deformações ocorridas segundo os eixos $\mathrm{AA}^{\prime}$ (fig. 5.8b) e BB' (fig. 6.8c), tem-se para os esforços internos resistentes:

$$
\begin{aligned}
& N_{I}=\int_{-h / 2}^{y_{0}} b_{w} \cdot \sigma_{c} \cdot d y-\int_{-h^{\prime} / 2}^{y_{0}} b_{w}^{\prime} \cdot \sigma_{c} \cdot d y+\sum_{i=1}^{n} \sigma_{s i} \cdot A_{s i} \\
& M_{I}=\int_{-h / 2}^{y_{w}} \cdot y \cdot \sigma_{c} \cdot d y-\int_{-h^{\prime} / 2}^{y_{0}} b_{w}^{\prime} \cdot \sigma_{c} \cdot y \cdot d y+\sum_{i=1}^{n} \sigma_{s i} \cdot A_{s i} \cdot Y_{s i}
\end{aligned}
$$




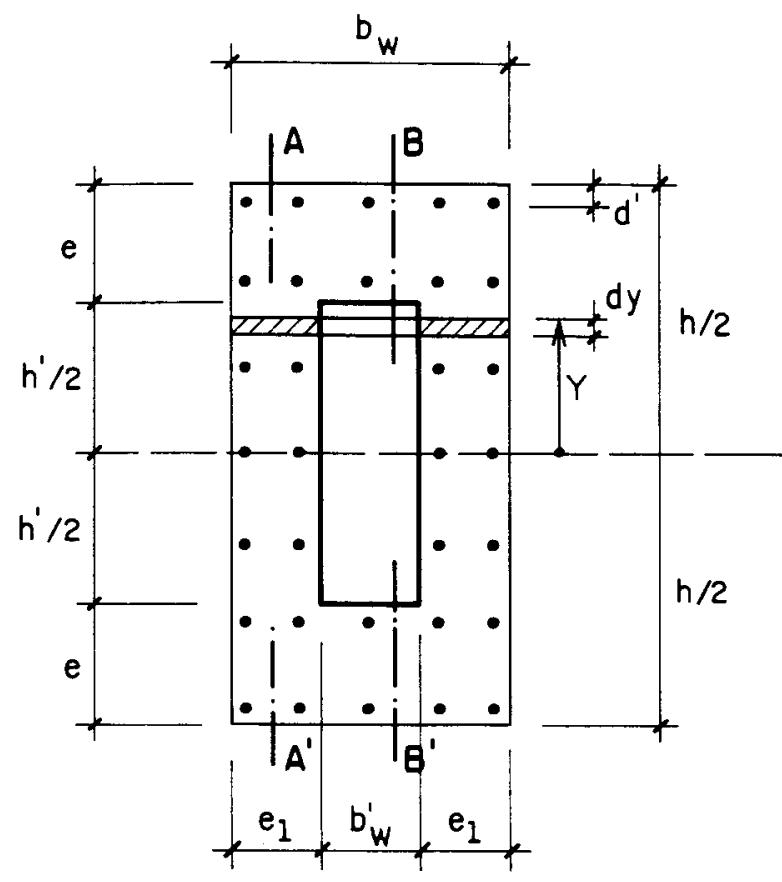

a)

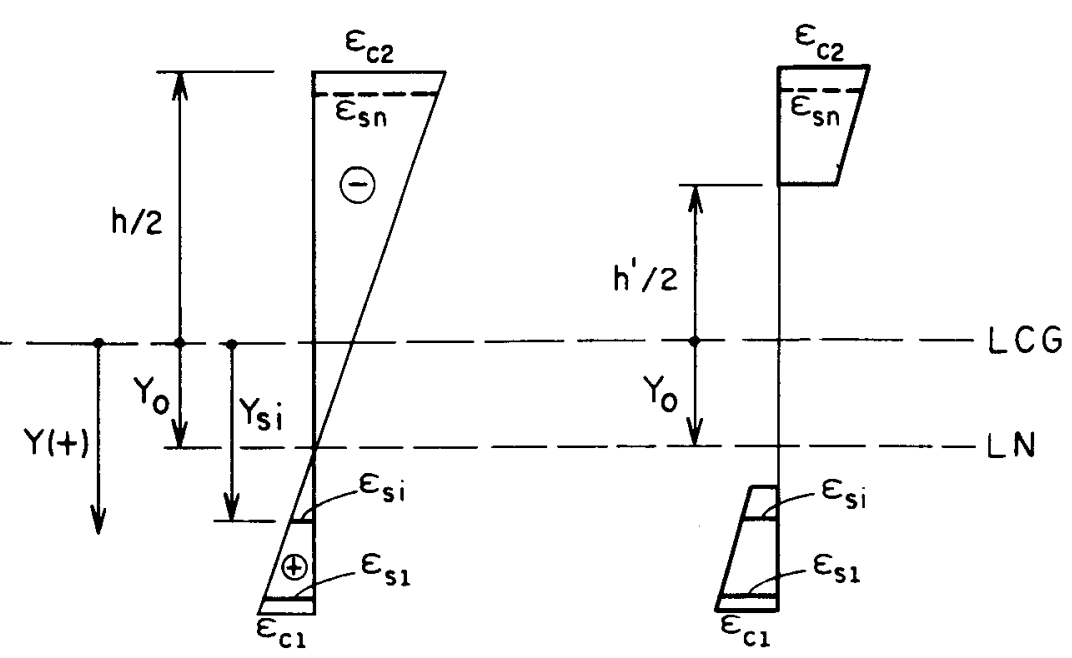

b)

c)

Fig. 6.8 - Deformações em uma seção retangular vazada de concreto armado.

\subsubsection{SEÇÕES CIRCULARES CHEIAS}

Considerando-se a seção circular da fig. $6.9 \mathrm{a}$ e as deformações ocorridas segundo o eixo $\mathrm{AA}^{\prime}$ (fig. 6.9b), lembrando que:

$$
\begin{aligned}
& R^{2}=x^{2}+y^{2} \\
& x=\sqrt{R^{2}-y^{2}}
\end{aligned}
$$

tem-se para os esforços internos resistentes:

$$
\begin{aligned}
& \mathrm{N}_{\mathrm{I}}=2 \int_{-\mathrm{R}}^{+\mathrm{R}} \sigma_{\mathrm{c}} \sqrt{\mathrm{R}^{2}-\mathrm{y}^{2}} \mathrm{dy}+\sum_{\mathrm{i}=1}^{\mathrm{n}} \sigma_{\mathrm{si}} \cdot \mathrm{A}_{\mathrm{si}} \\
& \mathrm{M}_{\mathrm{I}}=2 \int_{-\mathrm{R}}^{+\mathrm{R}} \sigma_{\mathrm{c}} \sqrt{\mathrm{R}^{2}-\mathrm{y}^{2}} \mathrm{dy}+\sum_{\mathrm{i}=1}^{\mathrm{n}} \sigma_{\mathrm{si}} \cdot \mathrm{A}_{\mathrm{si}} \cdot \mathrm{y}_{\mathrm{si}}
\end{aligned}
$$




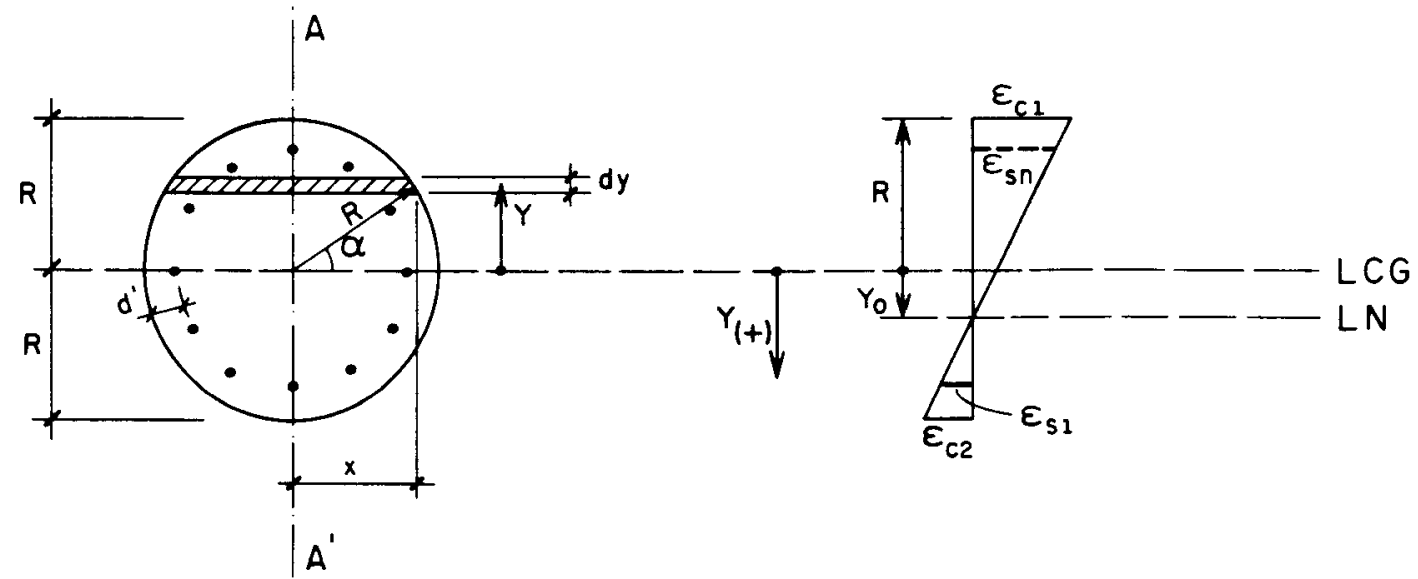

a)

b)

Fig. 6.9 - Deformações em uma seção circular de concreto armado.

\subsubsection{SEÇÕES CIRCULARES VAZADAS}

Considerando-se a seção circular vazada da fig. 6.10a e as deformações ocorridas segundo o eixo $\mathrm{AA}^{\prime}$ (fig. 6.10b), tem-se para os esforços internos resistentes:

$$
\begin{aligned}
& N_{I}=2 \int_{-R}^{+R} \sigma_{c} \sqrt{R^{2}-y^{2}} d y-2 \int_{-R^{\prime}}^{+R^{\prime}} \sigma_{R^{2}-y^{2}} d y+\sum_{i=1}^{n} \sigma_{s i} \cdot A_{s i} \\
& M_{I}=2 \int_{-R}^{+R} y \cdot \sigma_{c} \sqrt{R^{2}-y^{2}} d y-2 \int_{-R^{\prime}}^{+R^{\prime}} y \cdot \sigma_{c} \sqrt{R^{2}-y^{2}} d y+\sum_{i=1}^{n} \sigma_{s i} \cdot A_{s i} \cdot Y_{s i}
\end{aligned}
$$




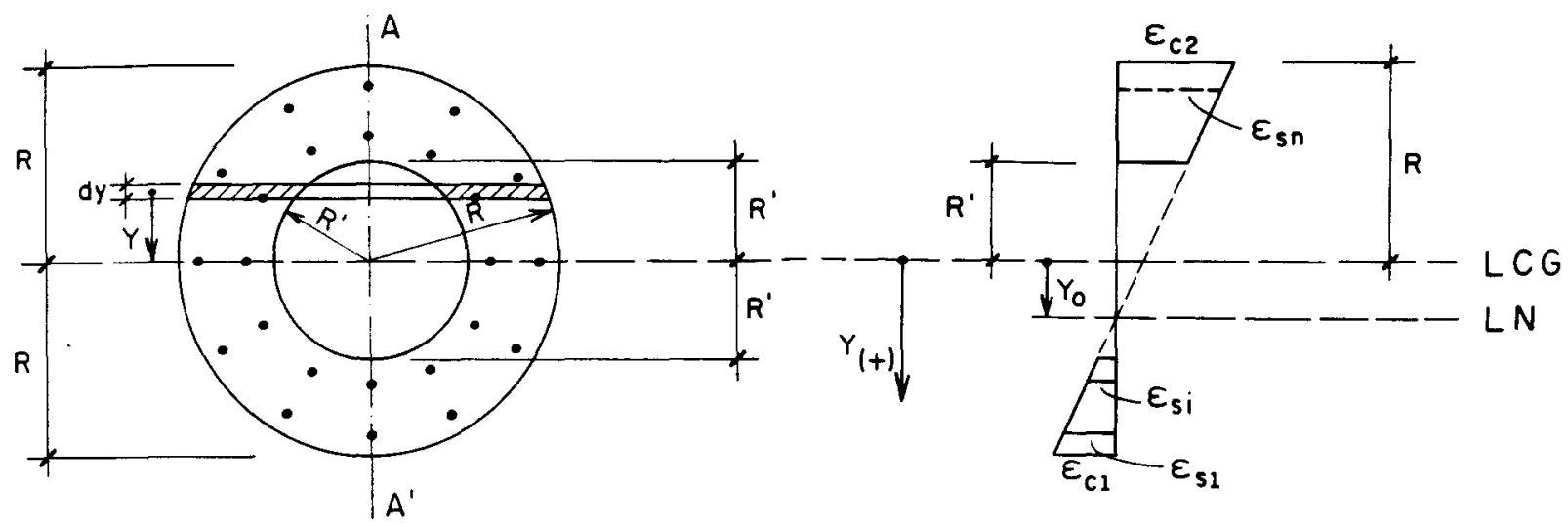

a)

b)

Fig. 6.10 - Deformações em uma seção circular vazada de concreto armado.

\subsection{EQUAÇÕES ADIMENSIONAIS DO EQUILÍBRIO}

É conveniente, para o estudo das seções de concreto armado, expressar as equações de equilíbrio da seção 6.2 na forma adimensional, independente das dimensões da peça e da tensão de cálculo. Os limites de integração serão transformados em parâmetros de integração, de forma análoga à das expressões $6.8 \mathrm{a}$ 6.10 , e a força normal resistente $\left(\mathrm{N}_{\mathrm{I}}\right)$ e o momento fletor resistente $\left(\mathrm{M}_{\mathrm{I}}\right)$ serão colocados na forma adimensional através das expressões 6.33 e 6.34, respectivamente, onde h é a maior altura da seção.

$$
\begin{aligned}
& u_{I}=\frac{N_{I}}{A_{c} \cdot f_{c d}} \\
& \mu_{I}=\frac{M_{I}}{A_{c} \cdot h \cdot f_{c d}}
\end{aligned}
$$

As expressões adimensionais serão desenvolvidas com base nos diagramas tensão-deformação do concreto - segundo a simplificação da NBR-6118/78 (fig. 4.4) 
e o CEB-1990 (fig. 4.5) - e nos parâmetros de integração apresentados nas figuras 6.11 e 6.12 .

As expressões adimensionais do equilibrio, para o esforço normal resistente $\left(v_{I}\right)$ e momento fletor resistente $\left(\mu_{\mathrm{I}}\right)$, permitem calcular a força normal e o momento fletor resistente em uma seção de concreto armado, em termos adimensionais, para uma dada curvatura e profundidade da linha neutra.

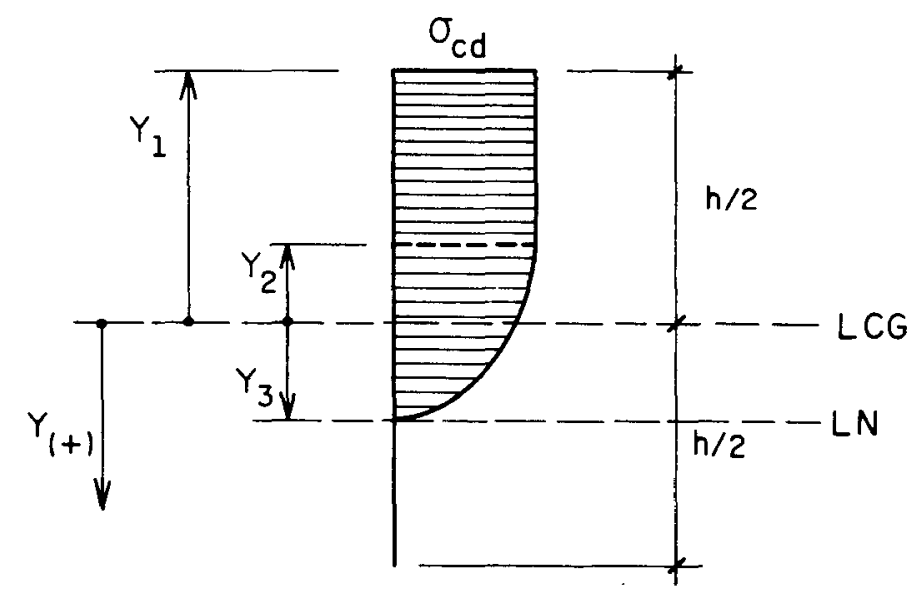

Fig. 6.11 - Tensões em uma seção de concreto armado diagrama parábola-retângulo.

Na figura 6.11 tem-se:

$\mathrm{y}_{1}=$ distância do eixo LCG até a borda mais comprimida

$\mathrm{y}_{2}=$ distância do eixo LCG até o ponto onde $\varepsilon_{\mathrm{c}}=-0,002$

$y_{3}=$ distância do eixo LCG até a linha neutra (LN).

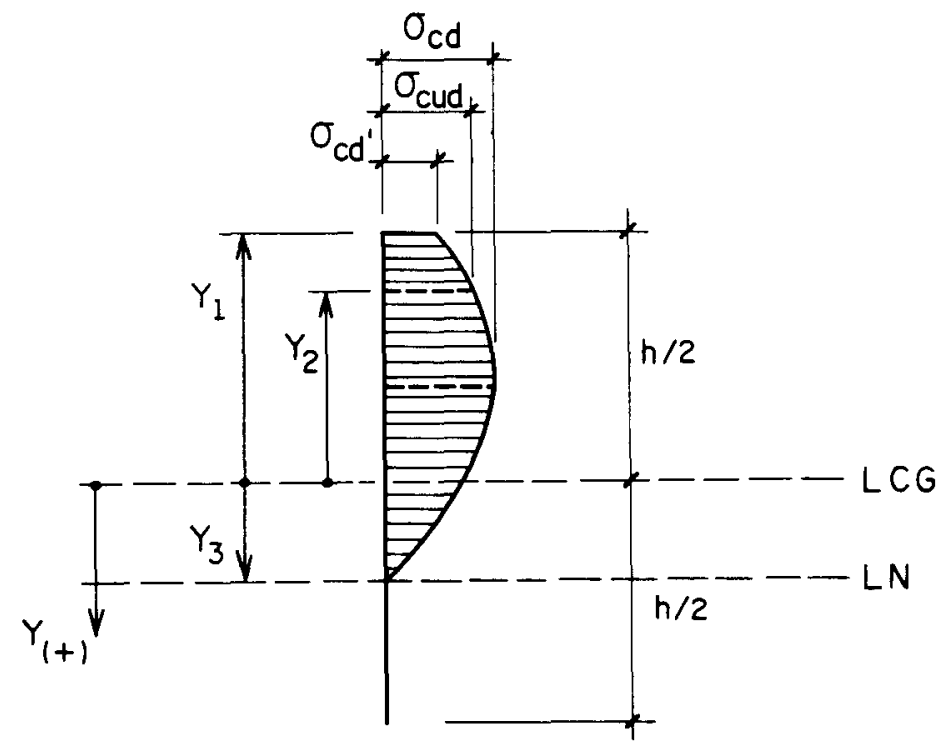

Fig. 6.12 - Tensões em uma seção de concreto armado diagrama CEB-1990. 
Na figura 6.12 tem-se:

$\mathrm{y}_{1}=$ distância do eixo LCG até a borda superior da seção

$\mathrm{y}_{2}=$ distância do eixo LCG até o ponto onde $\varepsilon_{\mathrm{c}}=\varepsilon_{\mathrm{cu}}$

$y_{3}=$ distância do eixo LCG até a linha neutra $(\mathrm{LN})$.

\subsubsection{SEÇÕES RETANGULARES CHEIAS}

Serão desenvolvidas expressões adimensionais para o cálculo da força normal e do momento fletor resistente utilizando, para as tensões e deformações do concreto, o diagrama parábola - retângulo e o diagrama proposto pelo CEB-1990.

\section{A) DIAGRAMA PARÁBOLA-RETÂNGULO}

Substituindo-se $\varepsilon_{\mathrm{cl}}$ por $-0,002$ na expressão (4.9), tem-se:

$$
\sigma_{\mathrm{c}}=0,85 \cdot \mathrm{f}_{\mathrm{cd}} \cdot\left[-1000 \cdot \varepsilon_{\mathrm{c}}-\left(-500 \cdot \varepsilon_{\mathrm{c}}\right)^{2}\right]
$$

Multiplicando-se a expressão 6.35 por -1 , para que resulte em uma tensão negativa para estar de acordo com a convenção apresentada na fig. 6.7 e colocandose $\varepsilon_{\mathrm{c}}$ (negativo para os encurtamentos) em evidência, tem-se:

$$
\sigma_{\mathrm{c}}=850 . \mathrm{f}_{\mathrm{cd}} \cdot\left[\varepsilon_{\mathrm{c}}\left(1+250 \cdot \varepsilon_{\mathrm{c}}\right)^{2}\right]
$$

que é a expressão das tensões no concreto, válida para o trecho parabólico do diagrama das tensões da fig. 6.11. Com base neste diagrama e nas expressões $6.36 \mathrm{e}$ 6.25 , a expressão da força normal resistente para a seção da fig. 6.7 a é obtida por:

$$
\begin{aligned}
& N_{I}=\int_{y_{1}}^{y_{2}} b_{w} \cdot\left(-0,85 \cdot f_{c d}\right) \cdot d y+\int_{y_{2}}^{y_{3}} b_{w} \cdot 850 \cdot f_{c d}\left[\varepsilon_{c} \cdot\left(1+250 \cdot \varepsilon_{c}\right)\right] \cdot d y+ \\
& \sum_{i=1}^{n} \sigma_{s i} \cdot A_{s i}
\end{aligned}
$$


Definindo-se $\omega$ - taxa mecânica de armadura da seção - e $\eta$ - relação entre a área de uma barra e a área total das barras da seção:

$$
\begin{aligned}
& \omega=\frac{f_{y d} \cdot A_{s}}{f_{c d} \cdot A_{c}} \\
& \eta=A_{s i} / A_{s}
\end{aligned}
$$

Efetuando-se as operações algébricas em 6.37, resolvendo as integrais, levando aos limites e utilizando as expressões 6.38 e 6.39 , tem-se:

$$
\begin{aligned}
& v_{\mathrm{I}}=-0,85\left(\beta_{2}-\beta_{1}\right)+850 \frac{\mathrm{h}}{\mathrm{r}}\left\{\frac{\left(\beta_{3}^{2}-\beta_{2}^{2}\right)}{2}+\left(\beta_{3}-\beta_{2}\right) \mathrm{k}_{1}+\right. \\
& \left.250 \frac{\mathrm{h}}{\mathrm{r}}\left[\frac{\left(\beta_{3}^{3}-\beta_{2}^{3}\right)}{3}+\left(\beta_{3}^{2}-\beta_{2}^{2}\right) \mathrm{k}_{1}+\left(\beta_{3}-\beta_{2}\right) \mathrm{k}_{1}^{2}\right]\right\}+ \\
& \frac{\omega}{\mathrm{f}_{\mathrm{yd}}} \sum_{\mathrm{i}=1}^{\mathrm{n}} \sigma_{\mathrm{si}} . \eta_{\mathrm{si}}
\end{aligned}
$$

Para a obtenção do momento fletor resistente adimensional, divide-se a expressão 6.26 por $A_{c} \cdot y \cdot f_{c d}$. Procedendo-se de maneira análoga à da obtenção da força normal resistente adimensional, tem-se:

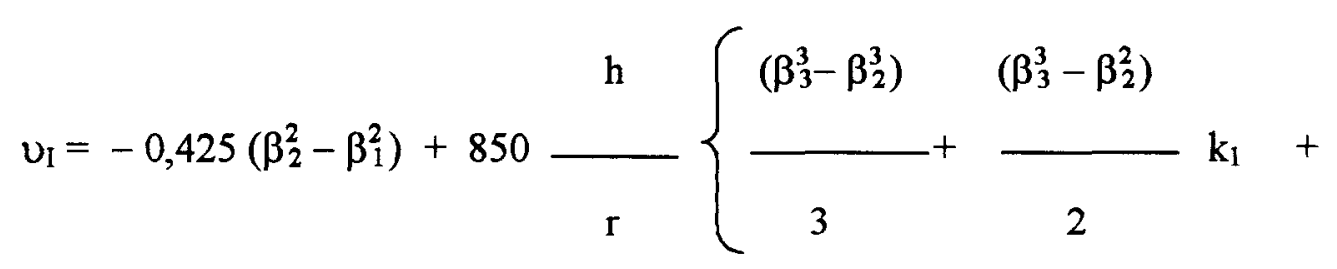




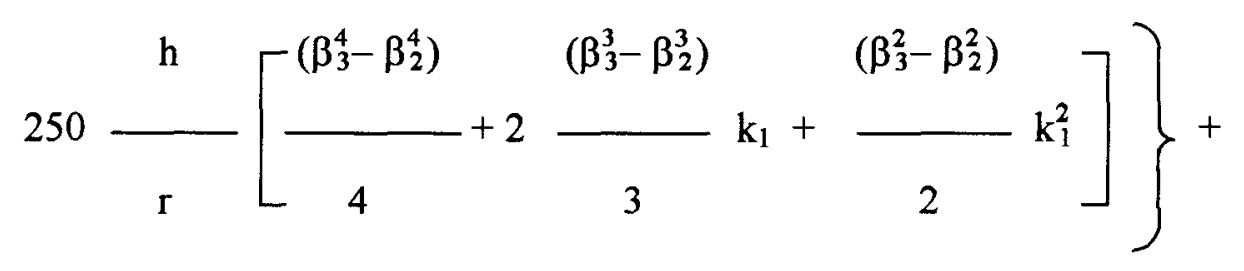

$\varlimsup_{f_{y d}}^{\infty} \sum_{i=1}^{n} \sigma_{s i} \cdot \eta_{s i} \cdot \beta_{s i}$

onde:

$k_{1}=1 / 2-\beta_{x}$

$\beta_{1}=y_{1} / h$

$\beta_{2}=y_{2} / h$

$\beta_{3}=y_{3} / h$

$\beta_{\mathrm{si}}=\mathrm{y}_{\mathrm{si}} / \mathrm{h}$.

B) DIAGRAMA DO CEB-1990

Substituindo-se $\varepsilon_{\mathrm{cl}}$ por $-0,0022$ na expressão 4.11 , tem-se:

$$
\begin{aligned}
& \sigma_{\mathrm{c}}=\left[\frac{454,55 \cdot \mathrm{k}_{2} \cdot \varepsilon_{\mathrm{c}}}{1+\left(-454,55 \cdot \mathrm{k}_{2}+909,09\right) \varepsilon_{\mathrm{c}}}+\right. \\
& \left.\frac{206.611,57 \cdot \varepsilon_{\mathrm{c}}^{2}}{1+\left(-454,55 \cdot \mathrm{k}_{2}+909,09\right) \varepsilon_{\mathrm{c}}}\right] \mathrm{f}_{\mathrm{cd}}
\end{aligned}
$$

onde $\mathrm{k}_{2}=\mathrm{E}_{\mathrm{cs}} / \mathrm{E}_{\mathrm{cl}}$. 
Substituindo-se $\varepsilon_{\mathrm{cl}}$ na expressão 4.14 , com $\xi=\mathrm{k}_{3}$ (ver expressão 4.15 ) e $\varepsilon_{\mathrm{cu}} / \varepsilon_{\mathrm{cl}}=\mathrm{k}_{4}$, para a parte descendente do diagrama $\sigma-\varepsilon$ com deformações maiores que $\varepsilon_{\mathrm{cu}}$, tem-se:

$$
\begin{aligned}
\sigma_{\mathrm{c}}=- & {\left[\left(\frac{\mathrm{k}_{3}}{\mathrm{k}}-\frac{2}{\mathrm{k}_{4}}\right) \cdot\left(206.611,57 \cdot \varepsilon_{\mathrm{c}}^{2}\right)+\right.} \\
& \left.\left(-\frac{4}{\mathrm{k}_{4}}-\mathrm{k}_{3}\right) \cdot\left(-454,55 \cdot \varepsilon_{\mathrm{c}}\right)\right]^{-1} \cdot \mathrm{f}_{\mathrm{cd}}
\end{aligned}
$$

Com base na figura 6.12 e substituindo 6.42 e 6.43 em 6.25 , tem-se:

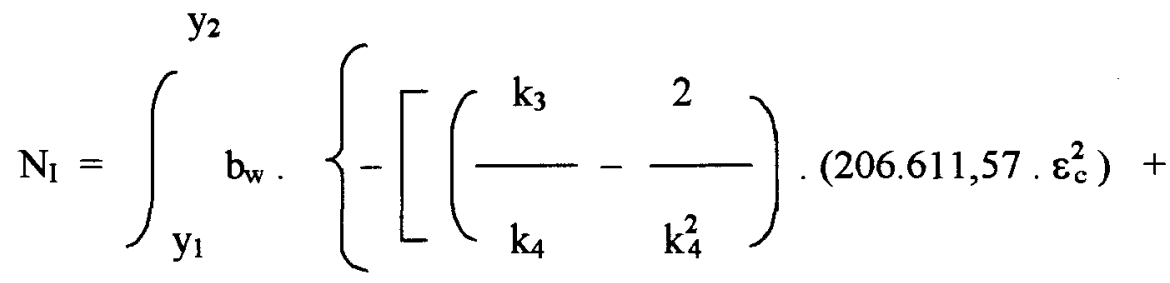

$$
\begin{aligned}
& \left.\left.\left(\frac{4}{k_{4}}-k_{3}\right) \cdot\left(-454,55 \cdot \varepsilon_{c}\right)\right]^{-1} \cdot f_{c d}\right\} \cdot d y+ \\
& \int_{y_{2}}^{y_{3}} b_{w} \cdot\left[\frac{454,55 \cdot k_{2} \cdot \varepsilon_{c}}{1+\left(-454,55 \cdot k_{2}+909,09\right) \varepsilon_{c}}+\right.
\end{aligned}
$$




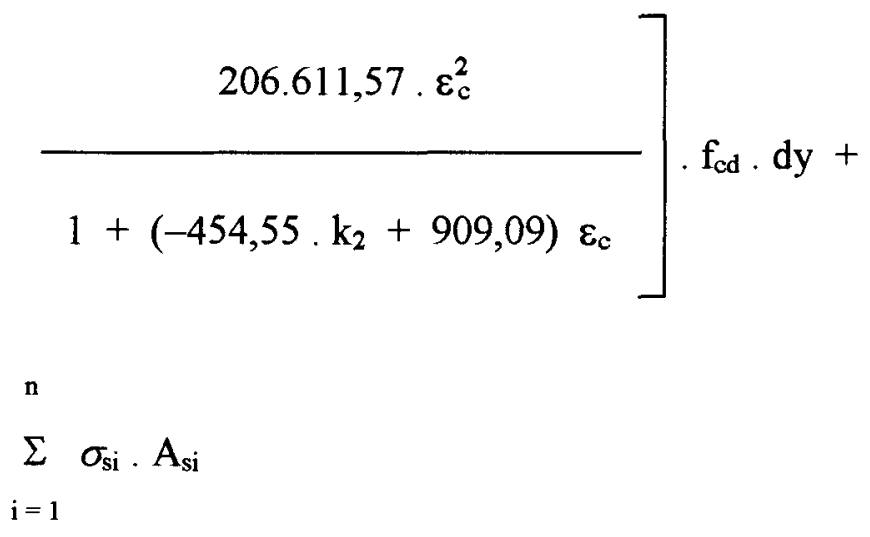

Dividindo a expressão 6.44 por $A_{c} \cdot f_{c d}$, substituindo $\varepsilon_{c}$ pela expressão 6.12 e colocando os limites de integração na forma dimensional, tem-se

$$
\begin{aligned}
& v_{I}=-\int_{k_{11} \cdot\left(\beta_{y}+k_{1}\right)^{2}+k_{12}\left(\beta_{y}+k_{1}\right)}^{d \beta_{y}}- \\
& \beta_{1} \\
& \beta_{3} \quad \beta_{3} \\
& \int \frac{\mathrm{k} 5\left(\beta_{\mathrm{y}}+\mathrm{k}_{1}\right)}{1+\mathrm{k}_{6}\left(\beta_{\mathrm{y}}+\mathrm{k}_{1}\right)} \mathrm{d} \beta_{\mathrm{y}}+\int \frac{\mathrm{k}_{7}\left(\beta_{\mathrm{y}}+\mathrm{k}_{1}\right)^{2}}{1+\mathrm{k}_{6}\left(\beta_{\mathrm{y}}+\mathrm{k}_{1}\right)} \mathrm{d} \beta_{\mathrm{y}}+ \\
& \beta_{2} \quad \beta_{2} \\
& \varlimsup_{\mathrm{f}_{\text {yd }}}^{\omega} \sum_{\mathrm{i}=1}^{\mathrm{n}} \sigma_{\text {si }} \cdot \eta_{\text {si }}
\end{aligned}
$$

Resolvendo-se as integrais da expressão 6.45 e levando-se aos limites, resulta: 


$$
v_{I}=-k_{5}\left[\begin{array}{c|c}
1 \\
k_{6}{ }^{2}
\end{array}\left(k_{6}\left(\beta_{3}-\beta_{2}\right)+l \eta\left|\begin{array}{c}
1+k_{6}\left(\beta_{2}+k_{1}\right) \\
1+k_{6}\left(\beta_{3}+k_{1}\right)
\end{array}\right|\right)\right]+
$$

$\frac{\mathrm{k}_{7}}{\mathrm{k}_{6}^{3}}\left\{\frac{\mathrm{k}_{6}}{2}\left[\mathrm{k}_{6}\left(\beta_{3}^{2}-\beta_{2}^{2}\right)+2 \mathrm{k}_{6} \mathrm{k}_{1}\left(\beta_{3}-\beta_{2}\right)\right]-\mathrm{k}_{6}\left(\beta_{3}-\beta_{2}\right)+\right.$

$\left.\ln \left|\frac{1+\mathrm{k}_{6}\left(\beta_{3}+\mathrm{k}_{1}\right)}{1+\mathrm{k}_{6}\left(\beta_{2}+\mathrm{k}_{1}\right)}\right|\right\}+-\operatorname{l\eta }\left|\frac{\frac{\mathrm{k}_{12}+\mathrm{k}_{11}\left(\beta_{1}+\mathrm{k}_{1}\right)}{\left(\beta_{1}+\mathrm{k}_{1}\right)}}{\mathrm{k}_{12}}\right|+$

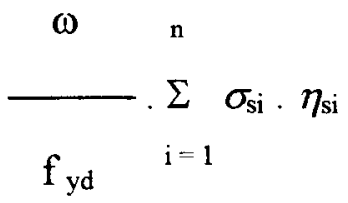

onde:

$$
\begin{aligned}
& \mathrm{k}_{5}=-454,55 \cdot \mathrm{k}_{2} \cdot \mathrm{h} / \mathrm{r} \\
& \mathrm{k}_{6}=\left(-454,55 \cdot \mathrm{k}_{2}+909,09\right) \cdot \mathrm{h} / \mathrm{r} \\
& \mathrm{k}_{7}=206 \cdot 611,57 \cdot(\mathrm{h} / \mathrm{r})^{2} \\
& \mathrm{k}_{11}=\frac{\mathrm{k}_{4} \cdot \mathrm{k}_{3}-2}{\mathrm{k}_{4}^{2}} \mathrm{k}_{7}
\end{aligned}
$$




$$
\mathrm{k}_{12}=\frac{4-\mathrm{k}_{4} \cdot \mathrm{k}_{3}}{\mathrm{k}_{4}}(-454,55 \cdot \mathrm{h} / \mathrm{r})
$$

Para a expressão do momento fletor resistente 6.26 , de acordo com o digrama de tensões da fig. 6.12, tem-se:

$$
\mathrm{M}_{\mathrm{I}}=\int_{\mathrm{y}_{1}}^{\mathrm{y}_{2}} \mathrm{~b}_{\mathrm{w}} \cdot \sigma_{\mathrm{c}} \cdot \mathrm{y} \cdot \mathrm{dy}+\int_{\mathrm{y}_{2}}^{\mathrm{y}_{3}} \mathrm{~b}_{\mathrm{w}} \cdot \sigma_{\mathrm{c}} \cdot \mathrm{y} \cdot \mathrm{dy}+\sum_{\mathrm{i}=1}^{\mathrm{n}} \sigma_{\mathrm{si}} \cdot \mathrm{y}_{\mathrm{si}} \cdot \mathrm{A}_{\mathrm{si}}
$$

Substituindo-se as expressões 6.42 e 6.43 na 6.47 , dividida por $A_{c} . h . f_{c d}$ e colocando-se os limites de integração na forma adimensional, resulta:

$$
\mu_{\mathrm{I}}=-\int_{\mathrm{k}_{11} \cdot\left(\beta_{\mathrm{y}}+\mathrm{k}_{1}\right)^{2}+\mathrm{k}_{12}\left(\beta_{\mathrm{y}}+\mathrm{k}_{1}\right)}^{\beta_{\mathrm{y}}} \mathrm{d} \beta_{\mathrm{y}}-
$$

$\beta_{3}$

$$
\begin{aligned}
& \beta_{3} \quad \beta_{3} \\
& \int \frac{\mathrm{k}_{5}\left(\beta_{\mathrm{y}}+\mathrm{k}_{1}\right) \cdot \beta_{\mathrm{y}}}{1+\mathrm{k}_{6} \cdot\left(\beta_{\mathrm{y}}+\mathrm{k}_{1}\right)} \mathrm{d} \beta \mathrm{y}+\int \frac{\mathrm{k}_{7}\left(\beta_{\mathrm{y}}+\mathrm{k}_{1}\right)^{2} \cdot \beta \mathrm{y}}{1+\mathrm{k}_{6} \cdot\left(\beta_{\mathrm{y}}+\mathrm{k}_{1}\right)} \mathrm{d} \beta_{\mathrm{y}}+ \\
& \beta_{2} \\
& \beta_{2}
\end{aligned}
$$




$$
+\frac{\omega}{\mathrm{f}_{\mathrm{yd}}} \cdot \sum_{\mathrm{i}=1}^{\mathrm{n}} \sigma_{\mathrm{si}} \cdot \beta_{\mathrm{si}} \cdot \eta_{\mathrm{si}}
$$

Resolvendo-se as integrais da expressão 6.48 e levando-se aos limites, resulta:

$$
\begin{aligned}
& \mu_{\mathrm{I}}=\frac{1}{\mathrm{k}_{12}}\left\{\left[\beta_{2} \cdot \ln \left|\frac{\mathrm{k}_{12}+\mathrm{k}_{11}\left(\beta_{2}+\mathrm{k}_{1}\right)}{\left(\beta_{2}+\mathrm{k}_{1}\right)}\right|-\beta_{1} \cdot \ln \mid \begin{array}{c}
\mathrm{k}_{12}+\mathrm{k}_{11}\left(\beta_{1}+\mathrm{k}_{1}\right) \\
\left(\beta_{1}+\mathrm{k}_{1}\right)
\end{array}\right]-\right. \\
& -1 \mathrm{k}_{12} \cdot \operatorname{l\eta }\left|\frac{\mathrm{k}_{12}+\mathrm{k}_{11}\left(\beta_{2}+\mathrm{k}_{1}\right)}{\mathrm{k}_{12}+\mathrm{k}_{11}\left(\beta_{1}+\mathrm{k}_{1}\right)}\right|+k_{11}\left(\beta_{2} \cdot \ln \mid \mathrm{k}_{12}+\mathrm{k}_{11}\left(\beta_{2}+\mathrm{k}_{1}\right)-\right. \\
& \left.\left.\beta_{1 .} l \eta\left|\mathrm{k}_{12}+\mathrm{k}_{1}\left(\beta_{1}+\mathrm{k}_{1}\right)\right|+\mathrm{k}_{1} l \eta\left|\frac{\mathrm{k}_{12}+\mathrm{k}_{11}\left(\beta_{2}+\mathrm{k}_{1}\right)}{\mathrm{k}_{12}+\mathrm{k}_{11}\left(\beta_{1}+\mathrm{k}_{1}\right)}\right|-\beta_{2}+\beta_{1}\right)\right]- \\
& \left.\beta_{2} \cdot \ln \left|\beta_{2}+\mathrm{k}_{1}\right|+\beta_{1} \cdot \ln \left|\beta_{1} \cdot \mathrm{k}_{1}\right|-\mathrm{k}_{1} \cdot \ln \left|\frac{\beta_{2}+\mathrm{k}_{1}}{\beta_{1}+\mathrm{k}_{1}}\right|+\beta_{2}-\beta_{1}\right\}- \\
& \mathrm{k}_{5}\left\{\frac{1}{\mathrm{k}_{6}^{3}}\left[\mathrm{k}_{6}^{2}\left(\beta_{3}^{2}-\beta_{2}^{2}\right)-\mathrm{k}_{13} \mathrm{k}_{6}\left(\beta_{3}-\beta_{2}\right)+\mathrm{k}_{13}^{2} \cdot \ln \mid \frac{\mathrm{k}_{13}+\mathrm{k}_{6} \beta_{3}}{\mathrm{k}_{13}+\mathrm{k}_{6} \beta_{2}}\right]\right]+
\end{aligned}
$$




$$
\left.\frac{\mathrm{k}_{1}}{\mathrm{k}_{6}^{2}}\left[\mathrm{k}_{6}\left(\beta_{3}-\beta_{2}\right)+\mathrm{k}_{13} \cdot l \eta\left|\frac{\mathrm{k}_{13}+\mathrm{k}_{6} \beta_{2}}{\mathrm{k}_{13}+\mathrm{k}_{6} \beta_{3}}\right|\right]\right\}+
$$

$\mathrm{k}_{7}\left\{\begin{array}{c}1 \\ 3 \mathrm{k}_{6}\end{array}\left(\beta_{3}^{3}-\beta_{2}^{3}\right)-\frac{\mathrm{k}_{13}}{\mathrm{k}_{6}^{4}}\left[\frac{\mathrm{k}_{6}^{2}}{2}\left(\beta_{3}^{2}-\beta_{2}^{2}\right)-\mathrm{k}_{13} \mathrm{k}_{6}\left(\beta_{3}-\beta_{2}\right)+\right.\right.$

$$
\left.\mathrm{k}_{13 .}^{2} \cdot \operatorname{l\eta }\left|\frac{\mathrm{k}_{13}+\mathrm{k}_{6} \beta_{3}}{\mathrm{k}_{13}+\mathrm{k}_{6} \beta_{2}}\right|\right]+\frac{2 \mathrm{k}_{1}}{\mathrm{k}_{6}^{3}}\left[\frac{\mathrm{k}_{6}^{3}}{2}\left(\beta_{3}^{2}-\beta_{2}^{2}\right)-\mathrm{k}_{13} \mathrm{k}_{6}\left(\beta_{3}-\beta_{2}\right)+\right.
$$

$\left.\mathrm{k}_{13}^{2} \cdot \ln \left|\frac{\mathrm{k}_{13}+\mathrm{k}_{6} \beta_{3}}{\mathrm{k}_{13}+\mathrm{k}_{6} \beta_{2}}\right|\right]+\frac{\mathrm{k}_{1}^{2}}{\mathrm{k}_{6}^{2}}\left[\mathrm{k}_{6}\left(\beta_{3}-\beta_{2}\right)+\right.$

$\left.\left.\mathrm{k}_{13} \cdot \ln \left|\frac{\mathrm{k}_{13}+\mathrm{k}_{6} \beta_{2}}{\mathrm{k}_{13}+\mathrm{k}_{6} \beta_{3}}\right|\right]\right\} \quad+$

$$
\frac{\omega}{\mathrm{f}_{\mathrm{yd}}} \cdot \sum_{\mathrm{i}=1}^{\mathrm{n}} \sigma_{\mathrm{si}} \cdot \beta_{\mathrm{si}} \cdot \eta_{\mathrm{si}}
$$

onde: $\mathrm{k}_{13}=1+\mathrm{k}_{1} \cdot \mathrm{k}_{6}$. 


\subsubsection{SEÇÕES RETANGULARES VAZADAS}

Para a determinação das expressões adimensionais de equilibrio, é necessário analisar as possibilidades de posicionamento dos pontos de mudança de curvatura dos diagramas $\sigma-\varepsilon$ do concreto. Os pontos de mudança podem estar localizados nos trechos de $\mathbf{A}$ a $\mathbf{D}$ da fig. 6.13, influindo nos limites de integração para as expressões destinadas ao cálculo da força normal adimensional e do momento fletor adimensional, uma vez que estes valores serão obtidos pela subtração das expressões correspondentes às seções das áreas do esquema mostrado na fig. 6.14.

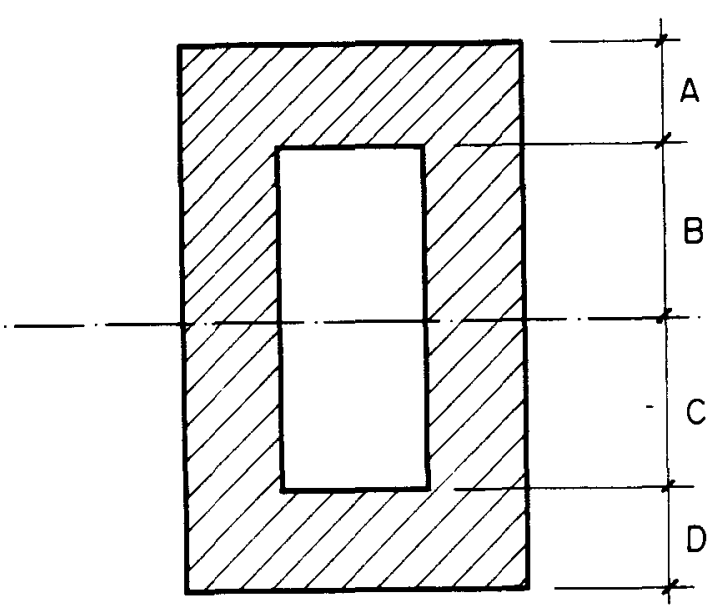

Fig. 6.13 - Trechos para a localização dos pontos $y_{2}$ e y3.

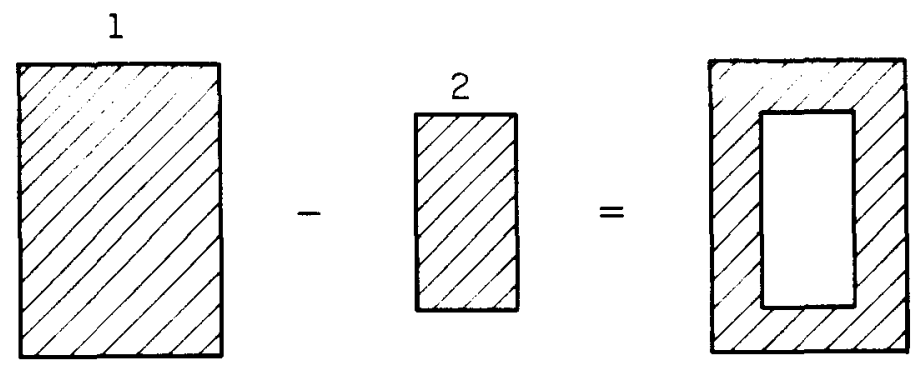

Fig. 6.14 - Esquema de cálculo das expressões adimensionais para uma seção vazada.

Com base nas seções da fig. 6.14, tem-se as expressões para o cálculo da força normal e momento fletor resistente, na forma genérica:

$$
\mathrm{N}_{\mathrm{I}}=\mathrm{R}_{\mathrm{C}_{1}}-\mathrm{R}_{\mathrm{C}_{2}}+\sum_{\mathrm{i}=1}^{\mathrm{n}} \mathrm{R}_{\mathrm{si}}
$$




$$
\mathbf{M}_{1}=\mathbf{M}_{\mathrm{C}_{1}}-\mathbf{M}_{\mathrm{C}_{2}}+\sum_{\mathrm{i}=1}^{\mathrm{n}} \mathbf{R}_{\mathrm{si}} \cdot \mathrm{y}_{\mathrm{si}}
$$

\section{A) DIAGRAMA PARÁBOLA-RETÂNGULO}

De acordo com as expressões $6.50,6.51$ e 4.9, com as figuras 6.15 a 6.17 , além da expressão 4.10 e da fig. 6.11 , tem-se:

$$
\begin{aligned}
& N_{I}=\int_{y_{1}}^{y_{2}} b_{w} \cdot\left(-0,85 \cdot f_{c d}\right) \cdot d y+\int_{y_{2}}^{y_{3}} b_{w} \cdot 850 \cdot f_{c d}\left[\varepsilon_{c} \cdot\left(1+250 \cdot \varepsilon_{c}\right)\right] \cdot d y- \\
& \int_{y_{e i}}^{y_{2}^{\prime}} b_{w}^{\prime} \cdot\left(-0,85 \cdot f_{c d}\right) \cdot d y-\int_{y_{2}^{\prime}}^{y^{\prime}{ }_{3}} b_{w}^{\prime} \cdot 850 \cdot f_{c d}\left[\varepsilon_{c} \cdot\left(1+250 \cdot \varepsilon_{c}\right)\right] \cdot d y+ \\
& \text { n } \\
& \Sigma \sigma_{\mathrm{si}} \cdot \mathrm{A}_{\mathrm{si}} \\
& \mathrm{i}=1 \\
& M_{1}=\int^{y_{2}} b_{w} \cdot\left(-0,85 \cdot f_{c d}\right) y \cdot d y+\int_{w}^{y_{3}} \cdot 850 \cdot f_{c d}\left[\varepsilon_{c} \cdot\left(1+250 \cdot \varepsilon_{c}\right)\right] y \cdot d y-
\end{aligned}
$$




$$
\int_{y_{e i}}^{y_{2}^{\prime}} b_{w}^{\prime} \cdot\left(-0,85 \cdot f_{c d}\right) y \cdot d y-\int_{y_{2}^{\prime}}^{y^{\prime}{ }^{\prime}}{ }^{\prime} \cdot 850 \cdot f_{c d}\left[\varepsilon_{c} \cdot\left(1+250 \cdot \varepsilon_{c}\right)\right] y \cdot d y+
$$

$$
\sum_{i=1}^{n} \sigma_{s i} \cdot A_{s i} \cdot y_{s i}
$$

onde:

$$
\begin{array}{llll}
y_{2}{ }_{2}=y_{e i} & \text { se } & y_{2} \leq y_{e i} & \text { (fig. 6.15a) } \\
y^{\prime}{ }_{2}=y_{2} & \text { se } & y_{2}>y_{e i} & \text { (fig. 6.15b) } \\
y^{\prime}{ }_{3}=y_{e s} & \text { se } & y_{3} \geq y_{e s} & \text { (fig. 6.16a) } \\
y^{\prime}{ }_{3}=y_{3} & \text { se } & y_{3}<y_{e s} & \text { (fig. 6.16b) } \\
y^{\prime}{ }_{3}=y_{3} & \text { se } & y_{3} \leq y_{e i} & \text { (fig. 6.17a) } \\
y^{\prime}{ }_{3}=y_{e i} & \text { se } & y_{3}>y_{e i} & \text { (fig. 6.17b) }
\end{array}
$$

As integrais das expressões 6.52 e 6.53 têm, para cada par e na forma adimensional, resolução idêntica à das expressões 6.40 e 6.41 , variando apenas os limites de integração.

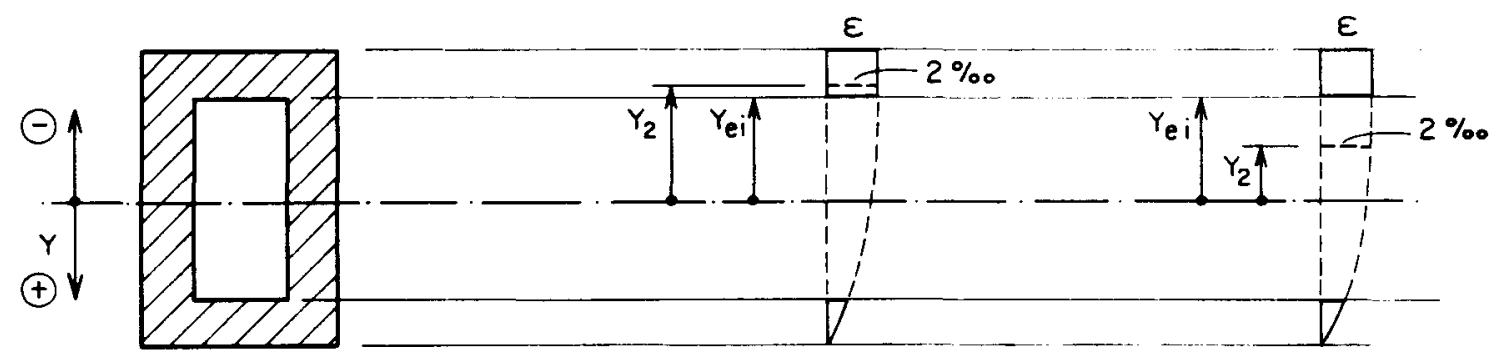

a)

b)

Fig. 6.15 - Possiveis localizações de y2. 


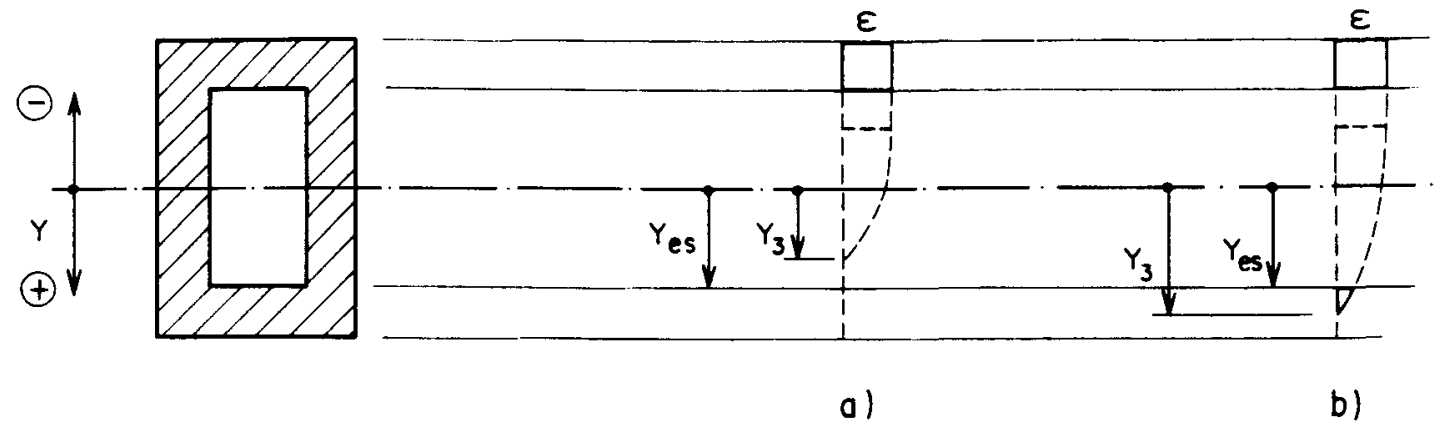

Fig. 6.16-Possíveis localizações de y3.

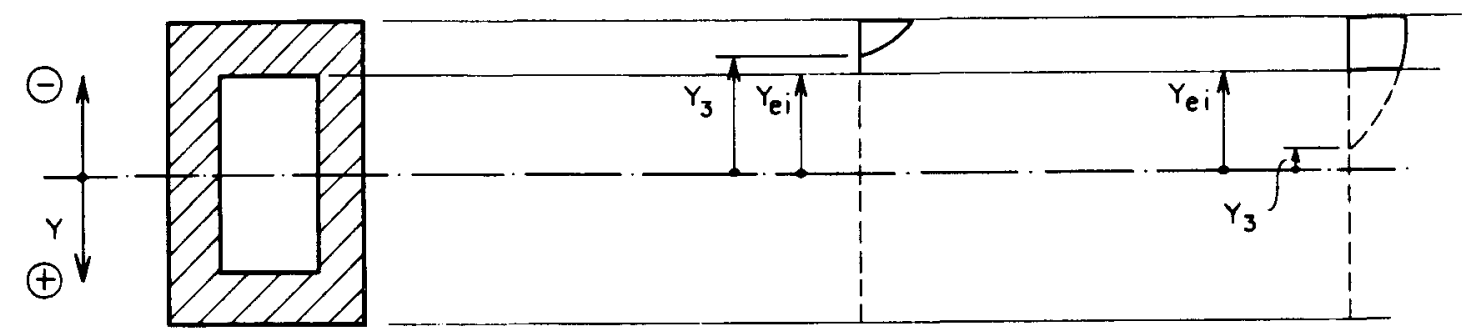

a)

b)

Fig. 6.17 - Possiveis localizações de $\mathrm{y}_{3}$ (negativo).

B) DIAGRAMA DO CEB-1990

São válidas as mesmas considerações feitas anteriormente, tendo-se para a forma adimensional:

$$
\begin{gathered}
v_{\mathrm{I}}=-\int_{\beta_{1}}^{\beta_{2}} \frac{\mathrm{d} \beta_{\mathrm{y}}}{\mathrm{k}_{11} \cdot\left(\beta_{\mathrm{y}}+\mathrm{k}_{1}\right)^{2}+\mathrm{k}_{12}\left(\beta_{\mathrm{y}}+\mathrm{k}_{1}\right)}- \\
\int \frac{\beta_{3} \mathrm{k}_{5}\left(\beta_{\mathrm{y}}+\mathrm{k}_{1}\right)}{\beta_{2}} \mathrm{~d} \beta_{\mathrm{y}}+\mathrm{k}_{6} \frac{\mathrm{k}_{7}\left(\beta_{\mathrm{y}}+\mathrm{k}_{1}\right)^{2}}{\beta_{2}} \mathrm{~d}+\mathrm{k}_{6} \cdot\left(\beta_{\mathrm{y}}+\mathrm{k}_{1}\right)
\end{gathered}
$$




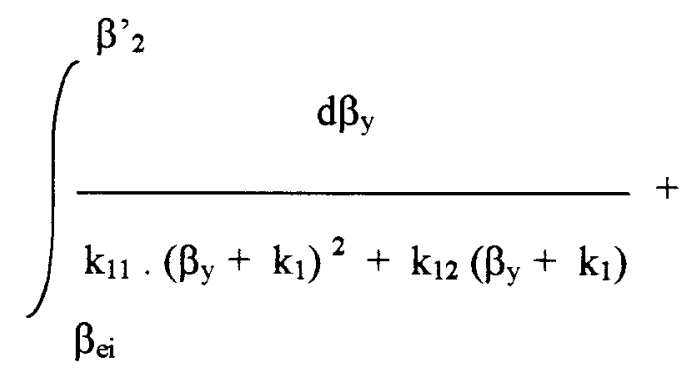

$\int_{\beta_{2}^{\prime}}^{\beta^{\prime} \mathrm{k}_{3}\left(\beta_{\mathrm{y}}+\mathrm{k}_{1}\right)} \int_{\beta_{2}}^{1+\mathrm{k}_{6} \cdot\left(\beta_{\mathrm{y}}+\mathrm{k}_{1}\right)} \mathrm{d} \beta_{\mathrm{y}}-\int_{\mathrm{k}_{7}\left(\beta_{\mathrm{y}}+\mathrm{k}_{1}\right)^{2}}^{\beta^{\prime}{ }_{3}} d \beta_{\mathrm{y}}+$

$\frac{\omega}{\mathrm{f}_{\mathrm{yd}}} \cdot \sum_{\mathrm{i}=1}^{\mathrm{n}} \sigma_{\mathrm{si}} \cdot \eta_{\mathrm{si}}$

$\mu_{\mathrm{I}}=-\int_{\beta_{1}}^{\beta_{2} \cdot\left(\beta_{\mathrm{y}}+\mathrm{k}_{1}\right)^{2}+\mathrm{k}_{12}\left(\beta_{\mathrm{y}}+\mathrm{k}_{1}\right)} \mathrm{d} \beta_{\mathrm{y}}-$

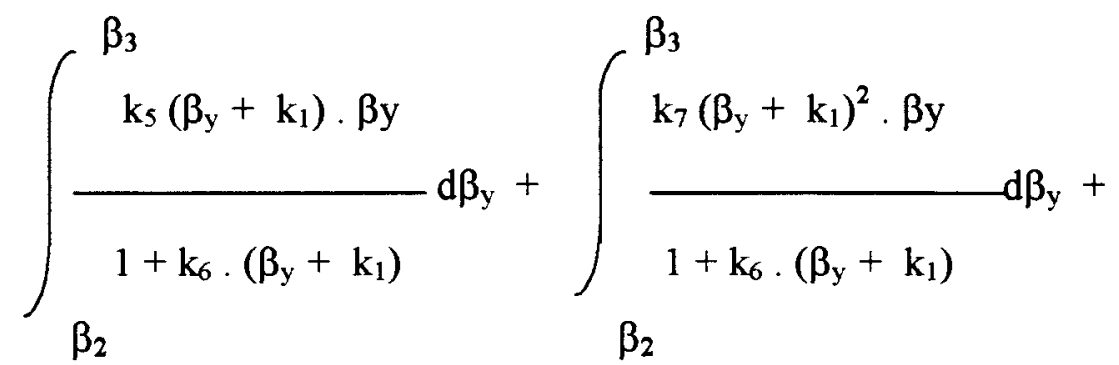




$$
\int_{k_{11} \cdot\left(\beta_{y}+k_{1}\right)^{2}+k_{12}\left(\beta_{y}+k_{1}\right)}^{\beta_{y}^{\prime}} d \beta_{y}+
$$

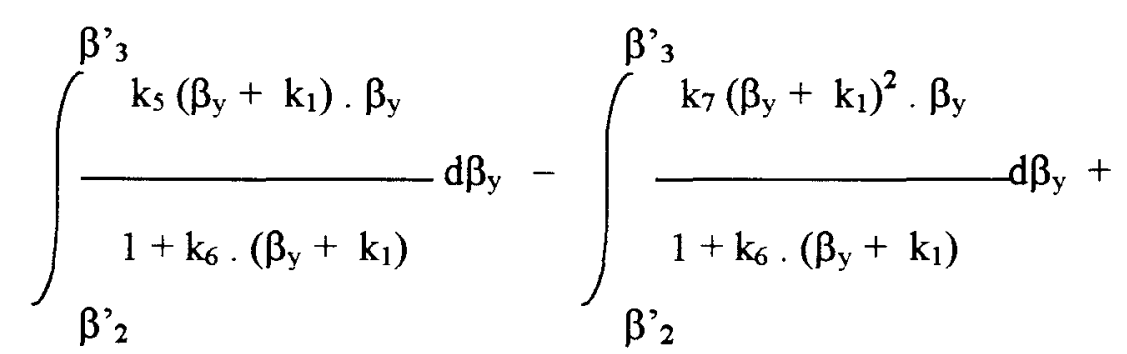

$$
\frac{\omega}{f_{y d}} \cdot \sum_{i=1}^{n} \sigma_{s i} \cdot \beta_{s i} \cdot \eta_{s i}
$$

onde:

$\beta^{\prime}{ }_{2}=y_{2}^{\prime} / h$

$\beta^{\prime}{ }_{3}=y^{\prime}{ }_{3} / h$

$\beta_{\mathrm{ei}}=\mathrm{y}_{\mathrm{ei}} / \mathrm{h}$.

As integrais das expressões 6.54 e 6.55 , três a três, têm solução igual às integrais do item 6.3.1.B.

\subsubsection{SEÇÕES CIRCULARES CHEIAS}

Os cálculos relativos a estas seções serão efetuados com base no raio das mesmas. Desta forma, as seções terão altura correspondente a $2 \mathrm{R}$ e, na forma adimensional, $\mathrm{R}$ é igual a 1 e $\beta_{\mathrm{y}}$ varia de $-1 \mathrm{a}+1$. 


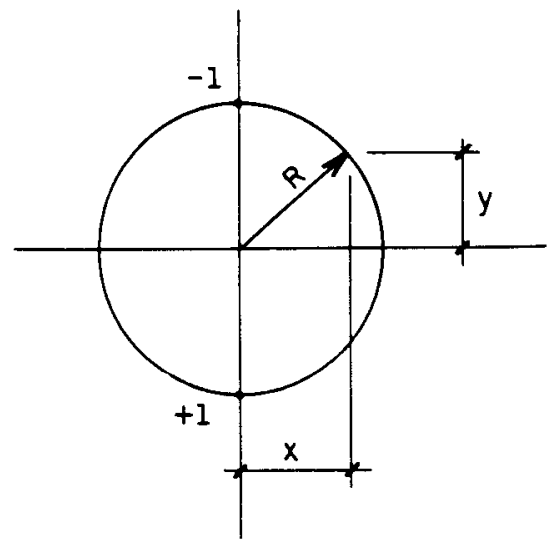

Fig. 6.18 - Seção circular de raio unitário.

\section{A) DIAGRAMA PARÁBOLA RETÂNGULO}

Tem-se, para a força normal resistente, com base na expressão 6.29 e nas figuras 6.11 e 6.18 :

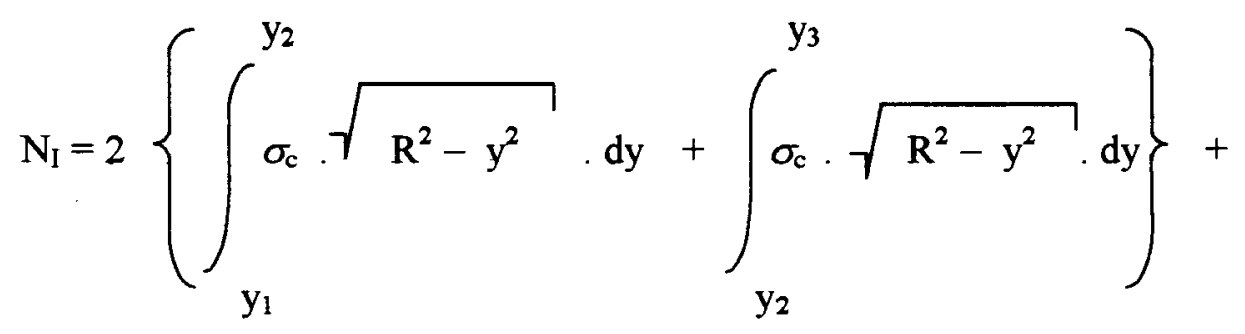

$\mathbf{n}$

$$
\sum_{\mathrm{i}=1} \sigma_{\mathrm{si}} \cdot \mathrm{A}_{\mathrm{si}}
$$

$\mathrm{Na}$ forma adimensional:

$$
v_{1}=\frac{2}{\pi}\left\{\int_{\beta_{1}}^{\beta_{2}}-0,85 \cdot \sqrt{1-\beta_{y}^{2}} \cdot d \beta_{y}+\right.
$$

$$
\left.\int_{\beta_{2}}^{\beta_{3}} 850 \cdot \varepsilon_{c}\left(1+250, \varepsilon_{c}\right) \sqrt{1-\beta_{y}^{2}} d \beta_{y}\right\}+
$$


$\underbrace{\omega}_{\mathrm{f}_{\mathrm{yd}}} \sum_{\mathrm{i}=1}^{\mathrm{n}} \sigma_{\mathrm{si}} \cdot \eta_{\mathrm{si}}$

Resultando:

$v_{I}=\frac{2}{\pi}\left\{-0,425\left(\beta_{2} \sqrt{1-\beta_{2}^{2}}-\beta_{1} \sqrt{1-\beta_{1}^{2}}+\operatorname{sen}^{-1} \beta_{2}-\operatorname{sen}^{-1} \beta_{1}\right)+\right.$

$850-\left[-\frac{1}{r}\left[\left(1-\beta_{3}^{2}\right)^{3 / 2}-\left(1-\beta_{2}^{2}\right)^{3 / 2}\right)+\underset{2}{-}\left[\beta_{3} \sqrt{1-\beta_{3}^{2}}-\right.\right.$

$\left.\beta_{2} \sqrt{1-\beta_{2}^{2}}+\operatorname{sen}^{-1} \beta_{3}-\operatorname{sen}^{-1} \beta_{2}\right)+$

$250-\left[-\left[\beta_{3}\left(2 \beta_{3}^{2}-1\right) \sqrt{1-\beta_{3}^{2}}-\beta_{2}\left(2 \beta_{2}^{2}-1\right) \sqrt{1-\beta_{2}^{2}}+\right.\right.$

$\left.\operatorname{sen}^{-1} \beta_{3}-\operatorname{sen}^{-1} \beta_{2}\right]-\frac{2 k_{1}}{3}\left(\left(1-\beta_{3}^{2}\right)^{3 / 2}-\left(1-\beta_{2}^{2}\right)^{3 / 2}\right)+$ 
$\left.\left.\overline{\mathrm{k}}_{1}^{2}\left[\beta_{3} \sqrt{1-\beta_{3}^{2}}-\beta_{2} \sqrt{1-\beta_{2}^{2}}+\operatorname{sen}^{-1} \beta_{3}-\operatorname{sen}^{-1} \beta_{2}\right)\right]\right]+$

$\underbrace{\omega}_{\mathrm{f}_{\mathrm{yd}}} \sum_{\mathrm{i}=1}^{\mathrm{n}} \sigma_{\mathrm{si}} \cdot \eta_{\mathrm{si}}$

De forma análoga, para o momento fletor resistente, na forma adimensional, tem-se:

$$
\begin{aligned}
& \mu_{\mathrm{I}}=\frac{2}{\pi}\left\{\int_{\beta_{1}}^{\beta_{2}}-0,85 \cdot \sqrt{1-\beta_{\mathrm{y}}^{2}} \cdot \beta_{\mathrm{y}} \cdot \mathrm{d} \beta_{\mathrm{y}}+\right. \\
& \left.\int_{\beta_{2}}^{\beta_{3}} 850 \cdot \varepsilon_{c}\left(1+250 \cdot \varepsilon_{c}\right) \sqrt{1-\beta_{y}^{2}} \cdot \beta_{y} \cdot d \beta_{y}\right\}+ \\
& \varlimsup_{f_{y d}}^{\omega} \cdot \sum_{i=1}^{n} \sigma_{s i} \cdot \eta_{s i} \cdot \beta_{s i}
\end{aligned}
$$

Resultando: 


$$
\begin{aligned}
& \mu_{\mathrm{I}}=\frac{2}{\pi}\left\{\frac{0,85}{3}\left[\left(1-\beta_{2}^{2}\right)^{3 / 2}-\left(1-\beta_{1}^{2}\right)^{3 / 2}\right]+\right. \\
& 850 \underset{\mathrm{h}}{\mathrm{r}}\left[-\left[\beta_{3}\left(2 \beta_{3}^{2}-1\right) \sqrt{1-\beta_{3}^{2}}-\beta_{2}\left(2 \beta_{2}^{2}-1\right) \sqrt{1-\beta_{2}^{2}}+\right.\right.
\end{aligned}
$$$$
\left.\operatorname{sen}^{-1} \beta_{3}-\operatorname{sen}^{-1} \beta_{2}\right]-\frac{\mathrm{k}_{1}}{3}\left[\left(1-\beta_{3}^{2}\right)^{3 / 2}-\left(1-\beta_{2}^{2}\right)^{3 / 2}\right]+
$$

$250-\left[-\frac{1}{\mathrm{r}}\left[\beta_{3}^{2} \sqrt{1-\beta_{3}^{2}}-\beta_{2}^{2} \sqrt{1-\beta_{2}^{2}}+2 \sqrt{1-\beta_{3}^{2}}-\right.\right.$

$\left.2 \sqrt{1-\beta_{2}^{2}}-3 \beta_{3}^{4} \sqrt{1-\beta_{3}^{2}}+3 \beta_{2}^{2} \sqrt{1-\beta_{2}^{2}}\right)+$

$\frac{k_{1}}{4}\left[\beta_{3}\left(2 \beta_{3}^{2}-1\right) \sqrt{1-\beta_{3}^{2}}-\beta_{2}\left(2 \beta_{2}^{2}-1\right) \sqrt{1-\beta_{2}^{2}}+\operatorname{sen}^{-1} \beta_{3}-\right.$

$$
\left.\left.\left.\operatorname{sen}^{-1} \beta_{2}\right]-\frac{k_{1}^{2}}{3}\left[\left(1-\beta_{3}^{2}\right)^{3 / 2}-\left(1-\beta_{2}^{2}\right)^{3 / 2}\right]\right]\right\}+
$$




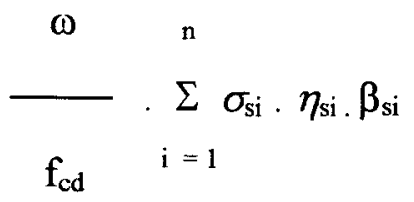

B) DIAGRAMA CEB-90

Substituindo-se $\sigma_{\mathrm{c}}$ na expressão 6.56 pelas expressões 6.42 e 6.43 e colocando na forma adimensional, tem-se para a força normal resistente adimensional:

$$
\mathrm{v}_{\mathrm{I}}=-\frac{2}{\pi}-\int_{\beta_{1}}^{\beta_{2}} \frac{\sqrt{1-\beta_{\mathrm{y}}^{2}}}{\mathrm{k}_{11} \cdot\left(\beta_{\mathrm{y}}+\mathrm{k}_{1}\right)^{2}+\mathrm{k}_{12} \cdot\left(\beta_{\mathrm{y}}+\mathrm{k}_{1}\right)} \mathrm{d} \beta_{\mathrm{y}}-
$$

$$
\mathrm{k}_{5} \int^{\beta_{3}} \frac{\left(\beta_{\mathrm{y}}+\mathrm{k}_{1}\right) \cdot \sqrt{1-\beta_{\mathrm{y}}^{2}}}{1+\mathrm{k}_{6} \cdot\left(\beta_{\mathrm{y}}+\mathrm{k}_{1}\right)} \mathrm{d} \beta_{\mathrm{y}}+
$$

$\beta_{2}$

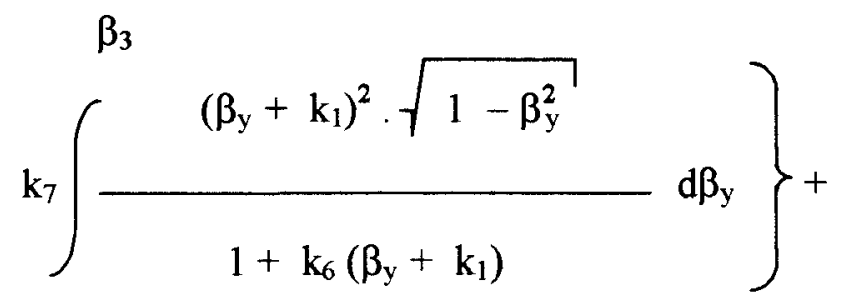

$\beta_{2}$ 
$\underbrace{\omega}_{\mathrm{f}_{\mathrm{yd}}} \cdot \sum_{\mathrm{i}=1}^{\mathrm{n}} \sigma_{\mathrm{si}} \cdot \eta_{\mathrm{si}} \cdot \beta_{\mathrm{si}}$

Integrando-se a expressão 6.61 por partes, tem-se:

$$
\begin{aligned}
& \mathrm{v}_{\mathrm{I}}=\frac{2}{\pi}\left\{\left[\frac{\left[\mathrm{k}_{\beta_{1}}^{\beta_{2}}\right.}{\mathrm{k}_{12}} \ln \frac{\mathrm{k}_{12}+\mathrm{k}_{11}\left(\beta_{\mathrm{y}}+\mathrm{k}_{1}\right)}{\left(\beta_{1}+\mathrm{k}_{1}\right)} \mid \mathrm{d} \beta_{\mathrm{y}}+\right.\right. \\
& \quad \mathrm{k}_{12} \int \frac{\ln \left|\mathrm{k}_{12}+\mathrm{k}_{11}\left(\beta_{\mathrm{y}}+\mathrm{k}_{1}\right)\right|}{\sqrt{1-\beta_{\mathrm{y}}^{2}}} \beta_{\mathrm{y}} \cdot \mathrm{d} \beta_{\mathrm{y}}-
\end{aligned}
$$$$
\left.\int \frac{\ln \left|\beta_{\mathrm{y}}+\mathrm{k}_{1}\right|}{\sqrt{1-\beta_{\mathrm{y}}^{2}}} \beta_{\mathrm{y}} \cdot \mathrm{d} \beta_{\mathrm{y}}\right]-\mathrm{k}_{5}\left[\frac { \beta _ { 3 } \sqrt { 1 - \beta _ { \mathrm { y } } ^ { 2 } } } { \beta _ { 2 } ^ { 2 } } \left[1+\mathrm{k}_{6} \cdot\left(\beta_{\mathrm{y}}+\mathrm{k}_{1}\right)-\right.\right.
$$$$
\left.l \eta\left|1+\mathrm{k}_{6} \cdot\left(\beta_{\mathrm{y}}+\mathrm{k}_{1}\right)\right|\right]+\frac{1}{\mathrm{k}_{6}^{2}}\left\{\int \frac{\beta_{\mathrm{y}}}{\sqrt{1-\beta_{\mathrm{y}}^{2}}} \mathrm{~d} \beta_{\mathrm{y}}+\right.
$$$$
\left.\mathrm{k}_{6} \int \frac{\beta_{\mathrm{y}}\left(\beta_{\mathrm{y}}+\mathrm{k}_{1}\right)}{\sqrt{1-\beta_{\mathrm{y}}^{2}}} \mathrm{~d} \beta_{\mathrm{y}}-\int \frac{\ln \left|1+\mathrm{k}_{6} \cdot\left(\beta_{\mathrm{y}}+\mathrm{k}_{1}\right)\right|}{\sqrt{1-\beta_{\mathrm{y}}^{2}}} \beta_{\mathrm{y} .} \mathrm{d} \beta_{\mathrm{y}}\right\}+
$$ 


$$
\mathrm{k}_{7} \frac{\sqrt{1-\beta_{\mathrm{y}}^{2}}}{\mathrm{k}_{6}^{3}}\left[-\left[1+\mathrm{k}_{6} \cdot\left(\beta_{\mathrm{y}}+\mathrm{k}_{1}\right)\right]^{2}-2\left[1+\mathrm{k}_{6} \cdot\left(\beta_{\mathrm{y}}+\mathrm{k}_{1}\right)\right]+\right.
$$

$$
\left.l \eta\left|1+\mathrm{k}_{6} \cdot\left(\beta_{\mathrm{y}}+\mathrm{k}_{1}\right)\right|\right]+\frac{1}{\mathrm{k}_{6}^{3}}\left\{\frac{1}{2} \int \frac{\left[1+\mathrm{k}_{6} \cdot\left(\beta_{\mathrm{y}}+\mathrm{k}_{1}\right)\right]^{2}}{\sqrt{1-\beta_{\mathrm{y}}^{2}}} \beta_{\mathrm{y}} \cdot \mathrm{d} \beta_{\mathrm{y}}-\right.
$$

$2 \int \frac{\left[1+\mathrm{k}_{6} \cdot\left(\beta_{\mathrm{y}}+\mathrm{k}_{1}\right)\right]}{\sqrt{1-\beta_{\mathrm{y}}^{2}}} \beta_{\mathrm{y}} \cdot \mathrm{d} \beta_{\mathrm{y}}+$

$$
\left.\left.\left.\int \frac{\ln \left|1+\mathrm{k}_{6} \cdot\left(\beta_{\mathrm{y}}+\mathrm{k}_{1}\right)\right|}{\sqrt{1-\beta_{\mathrm{y}}^{2}}} \beta_{\mathrm{y} \cdot \mathrm{d} \beta_{\mathrm{y}}}\right\}\right]\right\}^{+}
$$

$$
\underset{\mathrm{f}_{\mathrm{yd}}}{\omega} \cdot \sum_{\mathrm{i}=1}^{\mathrm{n}} \sigma_{\mathrm{si}} \cdot \eta_{\mathrm{si}}
$$

A primeira, segunda, quinta e oitava integrais da expressão 6.62 são resolvidas pela transformação do logaritmo neperiano para a forma de série de Taylor. De forma genérica, tem-se: 


$$
f(x)=f(a)+f^{\prime}(a)(x-a)+\frac{f^{\prime \prime}(a)}{2 !}(x-a)^{2}+\frac{f^{\prime \prime \prime}(a)}{3 !}(x-a)^{3}+
$$

$\mathbf{f}^{4)}(\mathrm{a})$

$-(\mathrm{x}-\mathrm{a})^{4}+$.

4 !

O valor de a é uma constante e deve ser positivo e diferente de zero, para que a série seja convergente, e diferente do valor de $\mathbf{x}$, para que a série não forneça valor nulo. Para uma convergência mais rápida - menos termos para o desenvolvimento em série da expressão 6.63 - o valor desta constante será fixado a cada iteração do cálculo dos diagramas momento fletor - força normal - curvatura. Tomando-se, como exemplo, o logaritmo neperiano da primeira integral da expressão 6.62:

$$
\ln \left|\mathrm{k}_{12}+\mathrm{k}_{11} \cdot\left(\beta_{\mathrm{y}}+\mathrm{k}_{1}\right)\right|=\operatorname{l\eta }|\mathrm{x}|
$$

A única variável de x é $\beta_{\mathrm{y}}$, variando de $-1 \mathrm{a}+1$ para seções circulares. Para a determinação da constante a, adota-se o valor de $\beta_{y}$ que conduza a um maior valor de $\mathrm{x}$, determinando-se a por:

$$
\begin{aligned}
& \mathrm{k}_{12}+\mathrm{k}_{11}\left( \pm 1+\mathrm{k}_{1}\right)=\mathrm{Z} \\
& \mathrm{a}=\mathrm{Z} \pm 1
\end{aligned}
$$

A expressão 6.65 destina-se à determinação de um valor para a constante a que não conduza a zero o logaritmo neperiano de um número diferente de 1 , adotando-se o maior valor (em módulo) obtido. Para o desenvolvimento em série da expressão 6.64 , tem-se:

$$
\ln \left|\mathrm{k}_{12}+\mathrm{k}_{11} \cdot\left(\beta_{\mathrm{y}}+\mathrm{k}_{1}\right)\right|=\operatorname{l\eta }|\mathrm{x}|=\operatorname{l\eta }|\mathrm{a}| \frac{(\mathrm{x}-\mathrm{a})^{0}}{0 !}+
$$




$$
\begin{aligned}
& \frac{1}{a} \cdot \frac{(x-a)^{1}}{1 !}-\frac{1}{a^{2}} \cdot \frac{(x-a)^{2}}{2 !}+\frac{2}{a^{3}} \cdot \frac{(x-a)^{3}}{3 !} \\
& l \eta|x|=C_{1}+C_{2}(x-a)-C_{3}(x-a)^{2}+C_{4}(x-a)^{3}
\end{aligned}
$$

Substituindo a expressão 6.66 na $1^{\underline{a}}$ integral da expressão 6.62 , com $\mathrm{k}_{8}=\mathrm{k}_{12}+\mathrm{k}_{11} \cdot \mathrm{k}_{1}-\mathrm{a}$, tem-se:

$\int_{\beta_{1}}^{\beta_{2}} \frac{\ln \left|\mathrm{k}_{12}+\mathrm{k}_{11} \cdot\left(\beta_{\mathrm{y}}+\mathrm{k}_{1}\right)\right|}{\sqrt{1-\beta_{\mathrm{y}}^{2}}} \beta_{\mathrm{y}} \cdot \mathrm{d} \beta_{\mathrm{y}}=\mathrm{C}_{1} \int_{\beta_{1}}^{\beta_{2}} \frac{\beta_{\mathrm{y}}}{\sqrt{1-\beta_{\mathrm{y}}^{2}}} \mathrm{~d} \beta_{\mathrm{y}}+$

$C_{2} \int_{\beta_{1}}^{\beta_{2}} \frac{\beta_{y}\left(k_{11} \cdot \beta_{y}+k_{8}\right)}{\sqrt{1-\beta_{y}^{2}}} d \beta_{y}-C_{3} \frac{\beta_{y}\left(k_{11} \cdot \beta_{y}+k_{8}\right)^{2}}{\sqrt{1-\beta_{y}^{2}}} d \beta_{y}+$

$\int_{\beta_{1}}^{\beta_{2}} \frac{\beta_{y}\left(k_{11} \cdot \beta_{y}+k_{8}\right)^{3}}{\sqrt{1-\beta_{y}^{2}}} d \beta_{y}$

Desenvolvendo as demais integrais da expressão 6.62 de forma análoga ao exposto, tem-se a expressão para a obtenção da força normal adimensional:

$$
\mathrm{u}_{\mathrm{I}}=-\frac{2}{\left[\mathrm{k}_{12}\right.} \cdot \frac{\sqrt{1-\beta_{\mathrm{y}}^{2}}}{\beta_{1}} \ln \left|\frac{\mathrm{k}_{12}+\mathrm{k}_{11}\left(\beta_{\mathrm{y}}+\mathrm{k}_{1}\right)}{\left(\beta_{\mathrm{y}}+\mathrm{k}_{1}\right)}\right| \mathrm{d} \beta_{\mathrm{y}}+
$$




$$
\begin{aligned}
& \frac{1}{\mathrm{k}_{12}}\left[-\mathrm{C}_{1} \sqrt{1-\beta_{\mathrm{y}}^{2}}+\mathrm{C}_{2}\left[\frac{\mathrm{k}_{11}}{2}\left(\operatorname{sen}^{-1} \beta_{\mathrm{y}}-\beta_{\mathrm{y}} \sqrt{1-\beta_{\mathrm{y}}^{2}}\right]-\right.\right. \\
& \left.\left.\mathrm{k}_{8} \sqrt{1-\beta_{\mathrm{y}}^{2}}\right)\right]-\mathrm{C}_{3}\left[-\mathrm{k}_{11}^{2}\left(\frac{\left(\beta_{\mathrm{y}}^{2}+2\right) \sqrt{1-\beta_{\mathrm{y}}^{2}}}{3}\right)+\right. \\
& \left.k_{11} \cdot k_{8}\left[\operatorname{sen}^{-1} \beta_{y}-\beta_{y} \sqrt{1-\beta_{y}^{2}}\right]-k_{8}^{2} \sqrt{1-\beta_{y}^{2}}\right]+
\end{aligned}
$$

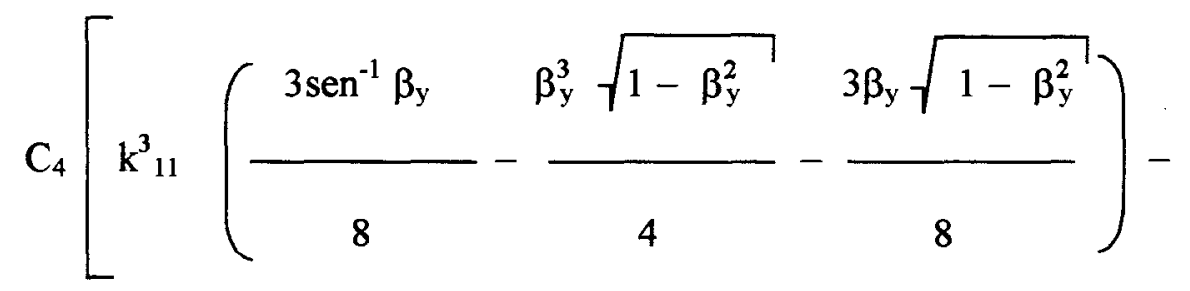

$$
\begin{aligned}
& 3 \mathrm{k}_{11}^{2} \mathrm{k}_{8}\left(\frac{\left(\beta_{\mathrm{y}}^{2}+2\right) \sqrt{1-\beta_{\mathrm{y}}^{2}}}{3}\right)+\frac{3 \mathrm{k}_{11} \mathrm{k}_{8}^{2}}{2}\left(\operatorname{sen}^{-1} \beta_{\mathrm{y}}-\beta_{\mathrm{y}} \sqrt{1-\beta_{\mathrm{y}}^{2}}\right)- \\
& \left.\mathrm{k}_{8}^{3} \sqrt{1-\beta_{\mathrm{y}}^{2}}\right]-\left[-\mathrm{C}_{5} \sqrt{1-\beta_{\mathrm{y}}^{2}}+\mathrm{C}_{6}\left[\frac { 1 } { 2 } \left[\operatorname{sen}^{-1} \beta_{\mathrm{y}}-\right.\right.\right.
\end{aligned}
$$




$$
\begin{aligned}
& \left.\left.\beta_{\mathrm{y}} \sqrt{1-\beta_{\mathrm{y}}^{2}}\right)-\mathrm{k}_{9} \sqrt{1-\beta_{\mathrm{y}}^{2}}\right]-\mathrm{C}_{7}\left[-\frac{\left(\beta_{\mathrm{y}}^{2}+2\right) \sqrt{1-\beta_{\mathrm{y}}^{2}}}{3}+\right. \\
& \left.\mathrm{k}_{9}\left[\operatorname{sen}^{-1} \beta_{\mathrm{y}}-\beta_{\mathrm{y}} \sqrt{1-\beta_{\mathrm{y}}^{2}}\right]-\mathrm{k}_{9}^{2} \sqrt{1-\beta_{\mathrm{y}}^{2}}\right]+ \\
& \mathrm{C}_{8}\left[\frac{3 \operatorname{sen}^{-1} \beta_{\mathrm{y}}}{8}-\frac{\beta_{\mathrm{y}}^{3} \sqrt{1-\beta_{\mathrm{y}}^{2}}}{4}-\frac{3 \beta_{\mathrm{y}} \sqrt{1-\beta_{\mathrm{y}}^{2}}}{8}-\right. \\
& 3 \mathrm{k}_{9}^{2}\left(\frac{\left(\beta_{\mathrm{y}}^{2}+2\right) \sqrt{1-\beta_{\mathrm{y}}^{2}}}{3}\right)+\frac{3 \mathrm{k}_{9}^{2}}{2}\left(\operatorname{sen}^{-1} \beta_{\mathrm{y}}-\beta_{\mathrm{y}} \sqrt{1-\beta_{\mathrm{y}}^{2}}\right)-
\end{aligned}
$$

$$
\left.\left.\mathrm{k}_{9}^{3} \sqrt{1-\beta_{\mathrm{y}}^{2}}\right]\right]-\mathrm{k}_{5}\left[\frac { \mathrm { k } _ { 6 } ^ { 2 } } { \sqrt { 1 - \beta _ { \mathrm { y } } ^ { 2 } } } \left[1+\mathrm{k}_{6}\left(\beta_{\mathrm{y}}+\mathrm{k}_{1}\right)-\right.\right.
$$




$$
\left.\operatorname{l\eta }\left|1+\mathrm{k}_{6}\left(\beta_{\mathrm{y}}+\mathrm{k}_{1}\right)\right|\right]+\frac{1}{\mathrm{k}_{6}^{2}}\left\{-\sqrt{1-\beta_{\mathrm{y}}^{2}}+\mathrm{k}_{6}\left[\beta_{\mathrm{y}}\left(\beta_{\mathrm{y}}+\mathrm{k}_{1}\right) \operatorname{sen}^{-1} \beta_{\mathrm{y}}-\right.\right.
$$$$
\left.\left(\beta_{y}^{2}-1\right) \operatorname{sen}^{-1} \beta_{y}-\frac{\beta_{y} \sqrt{1-\beta_{y}^{2}}}{2}-k_{1}\left(\beta_{y} \operatorname{sen}^{-1} \beta_{y}+\sqrt{1-\beta_{y}^{2}}\right)\right]-
$$

$$
\left[-C_{9} \sqrt{1-\beta_{y}^{2}}+C_{10}\left[\frac{k_{6}}{2}\left(\operatorname{sen}^{-1} \beta_{y}-\beta_{y} \sqrt{1-\beta_{y}^{2}}\right)-\right.\right.
$$

$$
\left.\left.\mathrm{k}_{10} \sqrt{1-\beta_{\mathrm{y}}^{2}}\right]\right]-\mathrm{C}_{11}\left[-\mathrm{k}_{6}^{2}\left[\frac{\left(\beta_{\mathrm{y}}^{2}+2\right) \sqrt{1-\beta_{\mathrm{y}}^{2}}}{3}\right]+\right.
$$

$$
\left.\mathrm{k}_{6} \mathrm{k}_{10}\left[\operatorname{sen}^{-1} \beta_{\mathrm{y}}-\beta_{\mathrm{y}} \sqrt{1-\beta_{\mathrm{y}}^{2}}\right]-\mathrm{k}^{2}{ }_{10} \sqrt{1-\beta_{\mathrm{y}}^{2}}\right]+
$$

$\mathrm{C}_{12}\left[\mathbf{k}_{6}^{3}\left(\frac{3 \operatorname{sen}^{-1} \beta_{\mathrm{y}}}{8}-\frac{\beta_{\mathrm{y}}^{3} \sqrt{1-\beta_{\mathrm{y}}^{2}}}{4}-\frac{3 \beta_{\mathrm{y}} \sqrt{1-\beta_{\mathrm{y}}^{2}}}{8}\right]-\right.$ 


$$
\begin{aligned}
& 3 \mathrm{k}_{6}^{2} \mathrm{k}_{10}\left(\frac{\left(\beta_{\mathrm{y}}^{2}+2\right) \sqrt{1-\beta_{\mathrm{y}}^{2}}}{3}\right)+\frac{3 \mathrm{k}_{6} \mathrm{k}_{10}^{2}}{2}\left(\operatorname{sen}^{-1} \beta_{\mathrm{y}}-\beta_{\mathrm{y}} \sqrt{1-\beta_{\mathrm{y}}^{2}}\right)- \\
& \left.\mathrm{k}_{10}^{3} \sqrt{1-\beta_{\mathrm{y}}^{2}}\right]+\mathrm{k}_{7}\left[\frac{\beta^{\beta_{2}}}{\mathrm{k}_{6}^{3}}\left[\begin{array}{l}
1 \\
\beta_{3}
\end{array}\right] \cdot\left[1+\mathrm{k}_{6}\left(\beta_{\mathrm{y}}+\mathrm{k}_{1}\right)\right]^{2}-\right. \\
& \left.2\left[1+\mathrm{k}_{6}\left(\beta_{\mathrm{y}}+\mathrm{k}_{1}\right)\right]+\ln \left|1+\mathrm{k}_{6}\left(\beta_{\mathrm{y}}+\mathrm{k}_{1}\right)\right|\right]+ \\
& \frac{1}{\mathrm{k}_{6}^{3}}\left\{\frac { 1 } { 2 } \left[-\mathrm{k}_{6} \mathrm{k}_{13}\left(\beta_{\mathrm{y}} \sqrt{1-\beta_{\mathrm{y}}^{2}}-\operatorname{sen}^{-1} \beta_{\mathrm{y}}\right)-\sqrt{1-\beta_{\mathrm{y}}^{2}} .\right.\right. \\
& \left.\left(\mathrm{k}_{13}^{2}+\frac{\mathrm{k}_{6}^{2}\left(\beta_{\mathrm{y}}^{2}+2\right)}{3}\right)\right]-\mathrm{k}_{6} \operatorname{sen}^{-1} \beta_{\mathrm{y}}+\left(\mathrm{k}_{6} \beta_{\mathrm{y}}+2 \mathrm{k}_{13}\right) \sqrt{1-\beta_{\mathrm{y}}^{2}}- \\
& C_{9} \sqrt{1-\beta_{y}^{2}}+C_{10}\left[\frac{k_{6}}{2}\left(\operatorname{sen}^{-1} \beta_{y}-\beta_{y} \sqrt{1-\beta_{y}^{2}}\right)-\right.
\end{aligned}
$$




$$
\begin{aligned}
& \left.\left.\mathrm{k}_{10} \sqrt{1-\beta_{\mathrm{y}}^{2}}\right)\right]-\mathrm{C}_{11}\left[-\mathrm{k}_{6}^{2}\left(\frac{\left(\beta_{\mathrm{y}}^{2}+2\right) \sqrt{1-\beta_{\mathrm{y}}^{2}}}{3}\right)+\right. \\
& \left.\mathrm{k}_{6} \mathrm{k}_{10}\left[\operatorname{sen}^{-1} \beta_{\mathrm{y}}-\beta_{\mathrm{y}} \sqrt{1-\beta_{\mathrm{y}}^{2}}\right]-\mathrm{k}_{10}^{2} \sqrt{1-\beta_{\mathrm{y}}^{2}}\right]+ \\
& \mathrm{C}_{12}\left[\mathrm{k}_{6}^{3}\left(\frac{3 \operatorname{sen}^{-1} \beta_{\mathrm{y}}}{8}-\frac{\beta_{\mathrm{y}}^{3} \sqrt{1-\beta_{\mathrm{y}}^{2}}}{4}-\frac{3 \beta_{\mathrm{y}} \sqrt{1-\beta_{\mathrm{y}}^{2}}}{8}\right]-\right.
\end{aligned}
$$

$$
3 \mathrm{k}_{6}^{2} \mathrm{k}_{10}\left(\frac{\left(\beta_{\mathrm{y}}^{2}+2\right) \sqrt{1-\beta_{\mathrm{y}}^{2}}}{3}\right)+\frac{3 \mathrm{k}_{6} \mathrm{k}^{2}{ }^{2}}{2}\left(\operatorname{sen}^{-1} \beta_{\mathrm{y}}-\beta_{\mathrm{y}} \sqrt{1-\beta_{\mathrm{y}}^{2}}\right)-
$$

$$
\left.\left.\left.\mathrm{k}^{3}{ }_{10} \sqrt{1-\beta_{\mathrm{y}}^{2}}\right]\right]\right\}+\underset{\mathrm{f}_{\mathrm{cd}}}{\sum_{\mathrm{i}=1}} \sigma_{\mathrm{si}} \cdot \eta_{\mathrm{si}}
$$


onde:

$\mathrm{C}_{1}, \mathrm{C}_{5}, \mathrm{C}_{9}=\ln |\mathrm{a}|$

$\mathrm{C}_{2}, \mathrm{C}_{6}, \mathrm{C}_{10}=1 / \mathrm{a}$

$\mathrm{C}_{3}, \mathrm{C}_{7}, \mathrm{C}_{11}=1 /\left(2 \mathrm{a}^{2}\right)$

$\mathrm{C}_{4}, \mathrm{C}_{8}, \mathrm{C}_{12}=1 /\left(3 \mathrm{a}^{3}\right)$

$\mathrm{k}_{8}=\mathrm{k}_{12}+\mathrm{k}_{11} \cdot \mathrm{k}_{1}-\mathrm{a}$

$\mathrm{k}_{9}=\mathrm{k}_{1}-\mathrm{a}$

$\mathrm{k}_{10}=\mathrm{k}_{13}-\mathrm{a}$

$\mathrm{k}_{13}=1+\mathrm{k}_{6} \cdot \mathrm{k}_{11}$.

Para o momento fletor adimensional, tem-se:

$$
\mu_{\mathrm{I}}=-2-\int_{\pi}^{\beta_{2}} \frac{\beta_{\mathrm{y}} \sqrt{1-\beta_{\mathrm{y}}^{2}}}{\mathrm{k}_{11} \cdot\left(\beta_{\mathrm{y}}+\mathrm{k}_{1}\right)^{2}+\mathrm{k}_{12} \cdot\left(\beta_{\mathrm{y}}+\mathrm{k}_{1}\right)} \mathrm{d} \beta_{\mathrm{y}}-
$$

$\int_{5}^{\beta_{3}} \frac{\beta_{y}\left(\beta_{y}+k_{1}\right) \cdot \sqrt{1-\beta_{y}^{2}}}{1+k_{6} \cdot\left(\beta_{y}+k_{1}\right)} d \beta_{y}+$

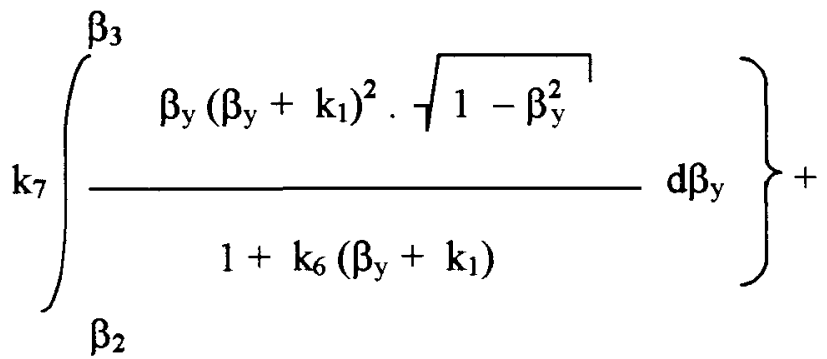




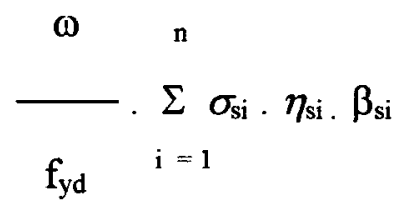

Resolvendo-se as integrais da expressão 6.69 , tem-se:

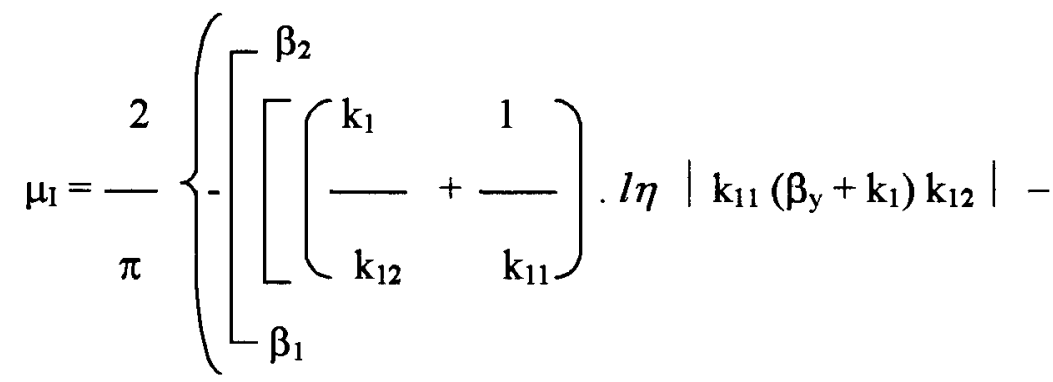

$\left.\left.\frac{\mathrm{k}_{1} \ln \left|\left(\beta_{\mathrm{y}}+\mathrm{k}_{1}\right)\right|}{\mathrm{k}_{12}}\right] \sqrt{1-\beta_{\mathrm{y}}^{2}}+\left(\frac{\mathrm{k}_{1}}{\mathrm{k}_{12}}+\frac{1}{\mathrm{k}_{11}}\right)\right] \cdot\left[-\mathrm{C}_{1} \sqrt{1-\beta_{\mathrm{y}}^{2}}+\right.$

$C_{2}\left[\frac{k_{11}}{2}\left(\operatorname{sen}^{-1} \beta_{y}-\beta_{y} \sqrt{1-\beta_{y}^{2}}\right)-\right.$

$\left.\mathrm{k}_{8} \sqrt{1-\beta_{\mathrm{y}}^{2}}\right]-\mathrm{C}_{3}\left[-\mathrm{k}_{11}^{2}\left(\frac{\left(\beta_{\mathrm{y}}^{2}+2\right) \sqrt{1-\beta_{\mathrm{y}}^{2}}}{3}\right)+\right.$ 


$$
\begin{aligned}
& \left.\mathrm{k}_{11} \mathrm{k}_{8}\left[\operatorname{sen}^{-1} \beta_{\mathrm{y}}-\beta_{\mathrm{y}} \sqrt{1-\beta_{\mathrm{y}}^{2}}\right]-\mathrm{k}_{8}^{2} \sqrt{1-\beta_{\mathrm{y}}^{2}}\right]+ \\
& \mathrm{C}_{4}\left[\mathrm{k}_{11}^{3}\left(\frac{3 \operatorname{sen}^{-1} \beta_{\mathrm{y}}}{8}-\frac{\beta_{\mathrm{y}}^{3} \sqrt{1-\beta_{\mathrm{y}}^{2}}}{4}-\frac{3 \beta_{\mathrm{y}} \sqrt{1-\beta_{\mathrm{y}}^{2}}}{8}\right]-\right. \\
& 3 \mathrm{k}_{11}^{2} \mathrm{k}_{8}\left(\frac{\left(\beta_{\mathrm{y}}^{2}+2\right) \sqrt{1-\beta_{\mathrm{y}}^{2}}}{3}\right)+\frac{3 \mathrm{k}_{11} \mathrm{k}_{8}^{2}}{2}\left(\operatorname{sen}^{-1} \beta_{\mathrm{y}}-\beta_{\mathrm{y}} \sqrt{1-\beta_{\mathrm{y}}^{2}}\right)- \\
& \left.\mathrm{k}_{8}^{3} \sqrt{1-\beta_{\mathrm{y}}^{2}}\right]-\frac{\mathrm{k}_{1}}{\mathrm{k}_{12}}\left[-\mathrm{C}_{5} \sqrt{1-\beta_{\mathrm{y}}^{2}}+\mathrm{C}_{6}\left[-\operatorname{sen}^{-1} \beta_{\mathrm{y}}-\right.\right. \\
& \left.\left.\beta_{\mathrm{y}} \sqrt{1-\beta_{\mathrm{y}}^{2}}\right]-\mathrm{k}_{9} \sqrt{1-\beta_{\mathrm{y}}^{2}}\right]+\mathrm{C}_{7}\left[\frac{\left(\beta_{\mathrm{y}}^{2}+2\right) \sqrt{1-\beta_{\mathrm{y}}^{2}}}{3}+\right. \\
& \left.k_{9}\left[\operatorname{sen}^{-1} \beta_{y}-\beta_{y} \sqrt{1-\beta_{y}^{2}}\right]-k_{9}^{2} \sqrt{1-\beta_{y}^{2}}\right]+
\end{aligned}
$$




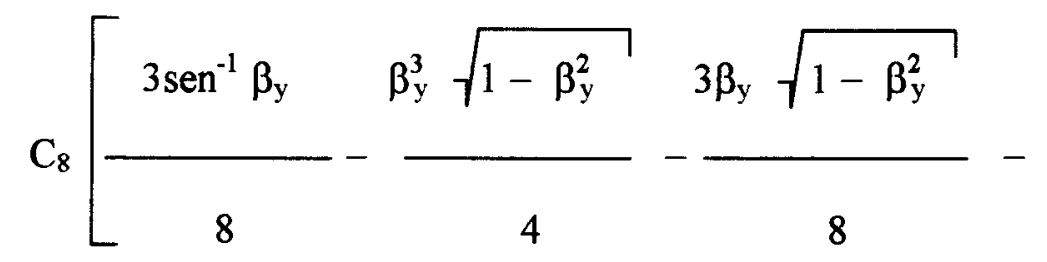

$3 \mathrm{k}_{9}^{2}\left(\frac{\left(\beta_{\mathrm{y}}^{2}+2\right) \sqrt{1-\beta_{\mathrm{y}}^{2}}}{3}\right)+\frac{3 \mathrm{k}_{9}^{2}}{2}\left(\operatorname{sen}^{-1} \beta_{\mathrm{y}}-\beta_{\mathrm{y}} \sqrt{1-\beta_{\mathrm{y}}^{2}}\right)-$

$\left.\left.\mathrm{k}_{9}^{3} \sqrt{1-\beta_{\mathrm{y}}^{2}}\right]\right]-\mathrm{k}_{5}\left[\begin{array}{l}\beta_{3} \\ \frac{1}{\mathrm{k}_{6}^{3}} \\ \beta_{2}\end{array}\right]\left(\frac{1}{2}\left(\mathrm{k}_{13}+\mathrm{k}_{6} \beta_{\mathrm{y}}\right)^{2}-\right.$

$\left.2 \mathrm{k}_{13}\left(\mathrm{k}_{13}+\mathrm{k}_{6} \beta_{\mathrm{y}}\right)+\mathrm{k}_{13}^{2} \ln \left|\mathrm{k}_{13}+\mathrm{k}_{6} \beta_{\mathrm{y}}\right|\right\} \cdot \sqrt{1-\beta_{\mathrm{y}}^{2}}-$

$\frac{1}{\mathbf{k}_{6}^{3}}\left\{-\frac{1}{2}\left[-\mathrm{k}_{13}^{2} \sqrt{1-\beta_{\mathrm{y}}^{2}}+\mathrm{k}_{13} \mathrm{k}_{6}\left(\operatorname{sen}^{-1} \beta_{\mathrm{y}}-\beta_{\mathrm{y}} \sqrt{1-\beta_{\mathrm{y}}^{2}}\right)-\right.\right.$

$\left.-\left(\beta_{y}^{2}+2\right) \sqrt{1-\beta_{y}^{2}}\right]+2 k_{3}\left[-k_{13} \sqrt{1-\beta_{y}^{2}}+\prod_{2}^{k_{6}^{2}}\left(\operatorname{sen}^{-1} \beta_{y}-\right.\right.$ 


$$
\begin{aligned}
& \left.\left.\beta_{\mathrm{y}} \sqrt{1-\beta_{\mathrm{y}}^{2}}\right]\right]-\mathrm{k}_{13}^{2}\left[-\mathrm{C}_{9} \sqrt{1-\beta_{\mathrm{y}}^{2}}+\mathrm{C}_{10}\left[-\mathrm{k}_{6}\left(\operatorname{sen}^{-1} \beta_{\mathrm{y}}-\right.\right.\right. \\
& \left.\left.\left.\beta_{\mathrm{y}} \sqrt{1-\beta_{\mathrm{y}}^{2}}\right]+\mathrm{k}_{10} \sqrt{1-\beta_{\mathrm{y}}^{2}}\right]\right]-\mathrm{C}_{11}\left[-\mathrm{k}_{6}^{2}\left(\frac{\left(\beta_{\mathrm{y}}^{2}+2\right) \sqrt{1-\beta_{\mathrm{y}}^{2}}}{3}\right)+\right. \\
& C_{12}\left[k_{6}^{3}\left(\frac{3 \operatorname{sen}^{-1} \beta_{y}}{8}-\frac{\beta_{y}^{3} \sqrt{1-\beta_{y}^{2}}}{4}-\frac{3 \beta_{y} \sqrt{1-\beta_{y}^{2}}}{8}\right]-\right.
\end{aligned}
$$$$
3 \mathrm{k}_{6} \mathrm{k}_{10}^{2}\left(\frac{\left(\beta_{\mathrm{y}}^{2}+2\right) \sqrt{1-\beta_{\mathrm{y}}^{2}}}{3}\right)+\frac{3 \mathrm{k}_{6} \mathrm{k}_{10}^{2}}{2}\left(\operatorname{sen}^{-1} \beta_{\mathrm{y}}-\beta_{\mathrm{y}} \sqrt{1-\beta_{\mathrm{y}}^{2}}\right)-
$$

$$
\left.\left.\mathrm{k}_{10}^{3} \sqrt{1-\beta_{\mathrm{y}}^{2}}\right]\right]+\mathrm{k}_{1}\left[\frac{1}{\mathrm{k}_{6}^{2}}\left[\mathrm{k}_{13}+\mathrm{k}_{6} \beta_{\mathrm{y}}-\mathrm{k}_{13} l \eta\left|\mathrm{k}_{13}+\mathrm{k}_{6} \beta_{\mathrm{y}}\right|\right] .\right.
$$




$$
\begin{aligned}
& \sqrt{1-\beta_{\mathrm{y}}^{2}}-\frac{1}{\mathrm{k}_{6}^{2}}\left[\mathrm{k}_{13} \sqrt{1-\beta_{\mathrm{y}}^{2}}-\frac{\mathrm{k}_{6}}{2}\left[\operatorname{sen}^{-1} \beta_{\mathrm{y}}-\right.\right. \\
& \left.\left.\beta_{\mathrm{y}} \sqrt{1-\beta_{\mathrm{y}}^{2}}\right)\right]+\mathrm{k}_{13}^{2}\left[-\mathrm{C}_{9} \sqrt{1-\beta_{\mathrm{y}}^{2}}+\mathrm{C}_{10}\left[\frac { \mathrm { k } _ { 6 } } { 2 } \left[\operatorname{sen}^{-1} \beta_{\mathrm{y}}-\right.\right.\right. \\
& \left.\left.\beta_{\mathrm{y}} \sqrt{1-\beta_{\mathrm{y}}^{2}}\right]-\mathrm{k}_{10} \sqrt{1-\beta_{\mathrm{y}}^{2}}\right]-\mathrm{C}_{11}\left[-\mathrm{k}_{6}^{2}\left(\frac{\left(\beta_{\mathrm{y}}^{2}+2\right) \sqrt{1-\beta_{\mathrm{y}}^{2}}}{3}\right)+\right. \\
& \left.\mathrm{k}_{6} \mathrm{k}_{10}\left[\operatorname{sen}^{-1} \beta_{\mathrm{y}}-\beta_{\mathrm{y}} \sqrt{1-\beta_{\mathrm{y}}^{2}}\right]-\mathrm{k}_{10}^{2} \sqrt{1-\beta_{\mathrm{y}}^{2}}\right]+ \\
& C_{12}\left[k_{6}^{3}\left(\frac{3 \operatorname{sen}^{-1} \beta_{y}}{8}-\frac{\beta_{y}^{3} \sqrt{1-\beta_{y}^{2}}}{4}-\frac{3 \beta y \sqrt{1-\beta_{y}^{2}}}{8}\right]-\right. \\
& 3 \mathrm{k}_{6} \mathrm{k}_{10}^{2}\left(\frac{\left(\beta_{\mathrm{y}}^{2}+2\right) \sqrt{1-\beta_{\mathrm{y}}^{2}}}{3}\right)+\frac{3 \mathrm{k}_{6} \mathrm{k}_{10}^{2}}{2}\left(\operatorname{sen}^{-1} \beta_{\mathrm{y}}-\beta_{\mathrm{y}} \sqrt{1-\beta_{\mathrm{y}}^{2}}\right)-
\end{aligned}
$$




$$
\begin{aligned}
& \left.\mathrm{k}_{10}^{3} \sqrt{1-\beta_{\mathrm{y}}^{2}}\right]+\mathrm{k}_{7}\left[\begin{array}{c}
\mathrm{k}_{3}^{2} \\
\mathrm{k}_{6}^{2} \\
\beta_{2}
\end{array} \frac{\ln \left|\mathrm{k}_{13}+\mathrm{k}_{6} \beta_{\mathrm{y}}\right| \sqrt{1-\beta_{\mathrm{y}}^{2}}}{6}+\right. \\
& \frac{1}{\mathrm{k}_{6}}\left[-\mathrm{C}_{9} \sqrt{1-\beta_{\mathrm{y}}^{2}}+\mathrm{C}_{10}\left[-\mathrm{k}_{2}^{\mathrm{k}_{6}}\left(\operatorname{sen}^{-1} \beta_{\mathrm{y}}-\beta_{\mathrm{y}} \sqrt{1-\beta_{\mathrm{y}}^{2}}\right)-\right.\right. \\
& \left.\left.\mathrm{k}_{10} \sqrt{1-\beta_{\mathrm{y}}^{2}}\right)\right]-\mathrm{C}_{11}\left[-\mathrm{k}_{6}^{2}\left(\frac{\left(\beta_{\mathrm{y}}^{2}+2\right) \sqrt{1-\beta_{\mathrm{y}}^{2}}}{3}\right)+\right. \\
& \left.k_{6} k_{10}\left[\operatorname{sen}^{-1} \beta_{y}-\beta_{y} \sqrt{1-\beta_{y}^{2}}\right]-k^{2}{ }_{10} \sqrt{1-\beta_{y}^{2}}\right]+ \\
& \mathrm{C}_{12}\left[\mathrm{k}_{6}^{3}\left(\frac{3 \operatorname{sen}^{-1} \beta_{\mathrm{y}}}{8}-\frac{\beta_{\mathrm{y}}^{3} \sqrt{1-\beta_{\mathrm{y}}^{2}}}{4}-\frac{3 \beta_{\mathrm{y}} \sqrt{1-\beta_{\mathrm{y}}^{2}}}{8}\right]-\right. \\
& 3 \mathrm{k}_{6}^{2} \mathrm{k}_{10}\left(\frac{\left(\beta_{\mathrm{y}}^{2}+2\right) \sqrt{1-\beta_{\mathrm{y}}^{2}}}{3}\right)+\frac{3 \mathrm{k}_{6} \mathrm{k}^{2}{ }_{10}}{2}\left(\operatorname{sen}^{-1} \beta_{\mathrm{y}}-\beta_{\mathrm{y}} \sqrt{1-\beta_{\mathrm{y}}^{2}}\right)-
\end{aligned}
$$




$$
\left.\left.\left.\mathrm{k}^{3}{ }_{10} \sqrt{1-\beta_{\mathrm{y}}^{2}}\right]\right]\right]+\frac{1}{\mathrm{k}_{6}}\left[\frac{\operatorname{sen}^{-1} \beta_{\mathrm{y}}}{8}-\frac{\beta_{\mathrm{y}}\left(1-\beta_{\mathrm{y}}^{2}\right)^{3 / 2}}{4}+\right.
$$

$$
\left.\frac{\beta_{\mathrm{y}} \sqrt{1-\beta_{\mathrm{y}}^{2}}}{8}\right]+\frac{\mathrm{k}_{13}}{3 \mathrm{k}_{6}^{2}}\left(1-\beta_{\mathrm{y}}^{2}\right)^{3 / 2}+\frac{\mathrm{k}^{2}{ }_{13}}{2 \mathrm{k}_{6}^{3}}\left[\beta_{\mathrm{y}} \sqrt{1-\beta_{\mathrm{y}}^{2}}+\right.
$$

$\left.\operatorname{sen}^{-1} \beta_{\mathrm{y}}\right)+2 \mathrm{k}_{1}\left\{\frac{1}{\mathrm{k}_{6}^{3}}\left[\frac{1}{2}\left(\mathrm{k}_{13}+\mathrm{k}_{6} \beta_{\mathrm{y}}\right)^{2}-\right.\right.$

$\left.2 \mathrm{k}_{13}\left(\mathrm{k}_{13}+\mathrm{k}_{6} \beta_{\mathrm{y}}\right)+\mathrm{k}_{13}^{2} \ln \left|\mathrm{k}_{13}+\mathrm{k}_{6} \beta_{\mathrm{y}}\right|\right\} \cdot \sqrt{1-\beta_{\mathrm{y}}^{2}}-$

$\frac{1}{\mathrm{k}_{6}^{3}}\left\{-\frac{1}{2}\left[-\mathrm{k}_{13}^{2} \sqrt{1-\beta_{\mathrm{y}}^{2}}+\mathrm{k}_{13} \mathrm{k}_{6}\left(\operatorname{sen}^{-1} \beta_{\mathrm{y}}-\beta_{\mathrm{y}} \sqrt{1-\beta_{\mathrm{y}}^{2}}\right)-\right.\right.$

$\left.\overline{\mathrm{k}}_{6}^{2}\left(\beta_{\mathrm{y}}+2\right) \sqrt{1-\beta_{\mathrm{y}}^{2}}\right]+2 \mathrm{k}_{3}\left[-\mathrm{k}_{13} \sqrt{1-\beta_{\mathrm{y}}^{2}}+\frac{\mathrm{k}_{6}}{2}-\left(\operatorname{sen}^{-1} \beta_{\mathrm{y}}-\right.\right.$ 
$\left.\left.\beta_{\mathrm{y}} \sqrt{1-\beta_{\mathrm{y}}^{2}}\right]\right]-\mathrm{k}_{13}^{2}\left[-\mathrm{C}_{9} \sqrt{1-\beta_{\mathrm{y}}^{2}}+\mathrm{C}_{10}\left[\begin{array}{c}\mathrm{k}_{6} \\ 2\end{array} \operatorname{sen}^{-1} \beta_{\mathrm{y}}-\right.\right.$

$\left.\left.\left.\beta_{\mathrm{y}} \sqrt{1-\beta_{\mathrm{y}}^{2}}\right]-\mathrm{k}_{10} \sqrt{1-\beta_{\mathrm{y}}^{2}}\right)\right]-\mathrm{C}_{11}\left[-\mathrm{k}_{6}^{2}\left(\frac{\left(\beta_{\mathrm{y}}^{2}+2\right) \sqrt{1-\beta_{\mathrm{y}}^{2}}}{3}\right]+\right.$

$\left.\mathrm{k}_{6} \mathrm{k}_{10}\left[\operatorname{sen}^{-1} \beta_{\mathrm{y}}-\beta_{\mathrm{y}} \sqrt{1-\beta_{\mathrm{y}}^{2}}\right]-\mathrm{k}_{10}^{2} \sqrt{1-\beta_{\mathrm{y}}^{2}}\right]+$

$\mathrm{C}_{12}\left[\mathrm{k}_{6}^{3}\left(\frac{3 \operatorname{sen}^{-1} \beta_{\mathrm{y}}}{8}-\frac{\beta_{\mathrm{y}}^{3} \sqrt{1-\beta_{\mathrm{y}}^{2}}}{4}-\frac{3 \beta \mathrm{y} \sqrt{1-\beta_{\mathrm{y}}^{2}}}{8}\right]-\right.$

$3 \mathrm{k}_{6}^{2} \mathrm{k}_{10}\left(\frac{\left(\beta_{\mathrm{y}}^{2}+2\right) \sqrt{1-\beta_{\mathrm{y}}^{2}}}{3}\right)+\frac{3 \mathrm{k}_{6} \mathrm{k}_{10}^{2}}{2}\left(\operatorname{sen}^{-1} \beta_{\mathrm{y}}-\beta_{\mathrm{y}} \sqrt{1-\beta_{\mathrm{y}}^{2}}\right)-$

$$
\left.\mathrm{k}_{10}^{3} \sqrt{1-\beta_{\mathrm{y}}^{2}}\right\}+\mathrm{k}_{1}\left[\frac{1}{\mathrm{k}_{6}^{2}}\left[\mathrm{k}_{13}+\mathrm{k}_{6} \beta_{\mathrm{y}}-\mathrm{k}_{13} \ln \left|\mathrm{k}_{13}+\mathrm{k}_{6} \beta_{\mathrm{y}}\right|\right] .\right.
$$




$$
\begin{aligned}
& \left.\mathrm{k}_{10}^{3} \sqrt{1-\beta_{\mathrm{y}}^{2}}\right\}+\mathrm{k}_{1}\left[-\frac{1}{\mathrm{k}_{6}^{2}}\left[\mathrm{k}_{13}+\mathrm{k}_{6} \beta_{\mathrm{y}}-\mathrm{k}_{13} l \eta\left|\mathrm{k}_{13}+\mathrm{k}_{6} \beta_{\mathrm{y}}\right|\right]\right. \\
& \sqrt{1-\beta_{\mathrm{y}}^{2}}-\frac{1}{\mathrm{k}_{6}^{2}}\left[\mathrm{k}_{13} \sqrt{1-\beta_{\mathrm{y}}^{2}}-\frac{\mathrm{k}_{6}}{2}-\left[\operatorname{sen}^{-1} \beta_{\mathrm{y}}-\right.\right. \\
& \left.\left.\beta_{\mathrm{y}} \sqrt{1-\beta_{\mathrm{y}}^{2}}\right]\right]+\mathrm{k}_{13}^{2}\left[-\mathrm{C}_{9} \sqrt{1-\beta_{\mathrm{y}}^{2}}+\mathrm{C}_{10}\left[\frac { \mathrm { k } _ { 6 } } { 2 } \left(\operatorname{sen}^{-1} \beta_{\mathrm{y}}-\right.\right.\right. \\
& \left.\left.\left.\beta_{\mathrm{y}} \sqrt{1-\beta_{\mathrm{y}}^{2}}\right]+\mathrm{k}_{10} \sqrt{1-\beta_{\mathrm{y}}^{2}}\right]\right]-\mathrm{C}_{11}\left[-\mathrm{k}_{6}^{2}\left(\frac{\left(\beta_{\mathrm{y}}^{2}+2\right) \sqrt{1-\beta_{\mathrm{y}}^{2}}}{3}\right)+\right. \\
& \mathrm{k}_{6} \mathrm{k}_{10}\left[\operatorname{sen}^{-1} \beta_{\mathrm{y}}-\beta_{\mathrm{y}} \sqrt{1-\beta_{\mathrm{y}}^{2}}\right]-\mathrm{k}^{2}{ }_{10} \sqrt{1-\beta_{\mathrm{y}}^{2}}+ \\
& C_{12}\left[\mathrm{k}_{6}^{3}\left(\frac{3 \operatorname{sen}^{-1} \beta_{\mathrm{y}}}{8}-\frac{\beta_{\mathrm{y}}^{3} \sqrt{1-\beta_{\mathrm{y}}^{2}}}{4}-\frac{3 \beta \mathrm{y} \sqrt{1-\beta_{\mathrm{y}}^{2}}}{8}\right]-\right.
\end{aligned}
$$


$3 \mathrm{k}_{6}^{2} \mathrm{k}_{10}\left(\frac{\left(\beta_{\mathrm{y}}^{2}+2\right) \sqrt{1-\beta_{\mathrm{y}}^{2}}}{3}\right)+\frac{3 \mathrm{k}_{6} \mathrm{k}_{10}^{2}}{2}\left(\operatorname{sen}^{-1} \beta_{\mathrm{y}}-\beta_{\mathrm{y}} \sqrt{1-\beta_{\mathrm{y}}^{2}}\right)-$

$$
\left.\left.\mathrm{k}_{10}^{3} \sqrt{1-\beta_{\mathrm{y}}^{2}}\right]\right]\left[\begin{array}{l}
\omega \\
\mathrm{f}_{\mathrm{cd}}
\end{array} \sum_{\mathrm{i}=1}^{\mathrm{n}} \sigma_{\mathrm{si}} \cdot \beta_{\mathrm{si}} \cdot \eta_{\mathrm{si}}\right.
$$

\subsubsection{SEÇÕES CIRCULARES VAZADAS}

São válidas as observações feitas na seção 6.3 .2 , sendo as resultantes de tensões obtidas pela subtração de valores esquematizados na fig. 6.14.

A) DIAGRAMA PARÁBOLA-RETÂNGULO

Tem-se, para a força normal resistente, na forma adimensional:

$$
v_{1}=\frac{2}{\pi}\left\{\int_{\beta_{1}}^{\beta_{2}}-0,85 \cdot \sqrt{1-\beta_{\mathrm{y}}^{2}} \cdot \mathrm{d} \beta_{\mathrm{y}}+\right.
$$

$\beta_{3}$

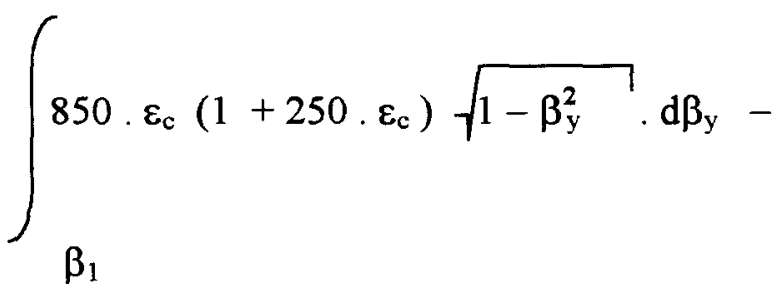




$$
\int_{-0,85 \cdot \sqrt{1-\beta_{y}^{2}} \cdot d \beta_{y}-}^{\beta_{2}}
$$

$\beta_{\mathrm{ei}}$

$$
\left.\int_{\beta_{2}^{\prime}}^{\beta^{\prime}{ }_{3}} 850 \cdot \varepsilon_{\mathrm{c}}\left(1+250 \cdot \varepsilon_{\mathrm{c}}\right) \sqrt{1-\beta_{\mathrm{y}}^{2}} \cdot \mathrm{d} \beta_{\mathrm{y}}\right\}+
$$

$$
+\frac{\omega}{f_{y d}} \sum_{i=1}^{n} \sigma_{s i} \cdot \eta_{s i}
$$

A resolução destas integrais, duas a duas, é igual à apresentada na seção 6.3.3.A, ocorrendo o mesmo para o momento fletor:

$$
\mu_{\mathrm{I}}=\frac{2}{\pi}\left\{\int_{\beta_{1}}^{\beta_{2}}-0,85 \cdot \beta_{\mathrm{y}} \sqrt{1-\beta_{\mathrm{y}}^{2}} \cdot \mathrm{d} \beta_{\mathrm{y}}+\right.
$$

$$
\int_{\beta_{2}}^{\beta_{3}} 850 \cdot \varepsilon_{c}\left(1+250 \cdot \varepsilon_{c}\right) \beta_{y} \sqrt{1-\beta_{y}^{2}} \cdot d \beta_{y}-
$$




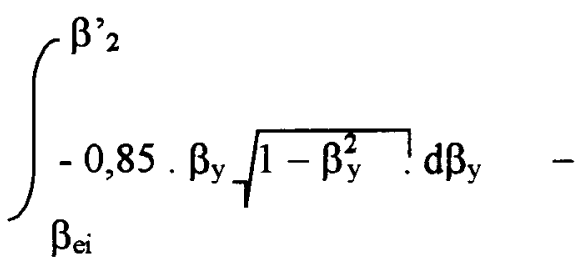

$\left.\int_{\beta_{2}^{\prime}}^{\beta_{2}^{\prime}{ }_{3}} 850 \cdot \varepsilon_{\mathrm{c}}\left(1+250 \cdot \varepsilon_{\mathrm{c}}\right) \beta_{\mathrm{y}} \sqrt{1-\beta_{\mathrm{y}}^{2}} \cdot d \beta_{\mathrm{y}}\right\}+$

$\omega \mathrm{n}$

$+\underbrace{}_{\mathrm{f}_{\mathrm{yd}}} \sum_{\mathrm{i}=1} \sigma_{\mathrm{si}} \cdot \eta_{\mathrm{si}} \cdot \beta_{\mathrm{si}}$

B) DIAGRAMA DO CEB-1990

Seguindo a forma já exposta, tem-se para a força normal resistente e momento fletor adimensionais:

$$
\mathrm{v}_{\mathrm{I}}=-2\left\{-\int_{\beta_{1}}^{\beta_{2}} \frac{\sqrt{1-\beta_{\mathrm{y}}^{2}}}{\mathrm{k}_{11} \cdot\left(\beta_{\mathrm{y}}+\mathrm{k}_{1}\right)^{2}+\mathrm{k}_{12}\left(\beta_{\mathrm{y}}+\mathrm{k}_{1}\right)} \mathrm{d} \beta_{\mathrm{y}}-\right.
$$

$$
\int_{\beta_{2}}^{\beta_{3}} \frac{\left(\beta_{y}+k_{1}\right) \cdot \sqrt{1-\beta_{y}^{2}}}{1+k_{6} \cdot\left(\beta_{y}+k_{1}\right)} d \beta_{y}+
$$


$\mathrm{k}_{7} \frac{\left(\beta_{\mathrm{y}}+\mathrm{k}_{1}\right)^{2} \cdot \sqrt{1-\beta_{\mathrm{y}}^{2}}}{1+\mathrm{k}_{6} \cdot\left(\beta_{\mathrm{y}}+\mathrm{k}_{1}\right)} \mathrm{d} \beta_{\mathrm{y}}+$

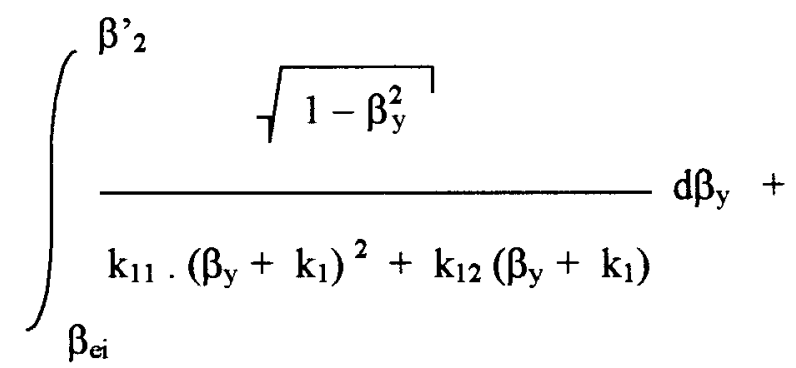

$\mathrm{k}_{5} \frac{\left(\beta_{\mathrm{y}}+\mathrm{k}_{1}\right) \cdot \sqrt{1-\beta_{\mathrm{y}}^{2}}}{\beta_{\beta_{2}}^{\prime}} \mathrm{d} \mathrm{k}_{\mathrm{y}}-$

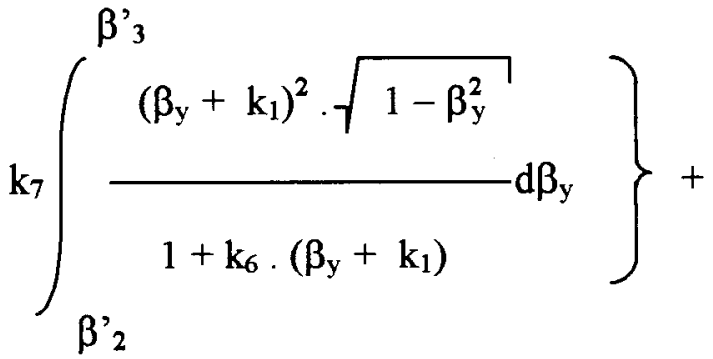

$\frac{\omega}{f_{y d}} \cdot \sum_{i=1}^{n} \sigma_{s i} \cdot \eta_{s i}$ 


$$
\mu_{\mathrm{I}}=-2\left\{-\int_{\beta_{1}}^{\beta_{2}} \frac{\beta_{\mathrm{y}} \sqrt{1-\beta_{\mathrm{y}}^{2}}}{\mathrm{k}_{11} \cdot\left(\beta_{\mathrm{y}}+\mathrm{k}_{1}\right)^{2}+\mathrm{k}_{12}\left(\beta_{\mathrm{y}}+\mathrm{k}_{1}\right)} \mathrm{d} \beta_{\mathrm{y}}-\right.
$$$$
\int_{\beta_{2}}^{\beta_{3}} \frac{\beta_{\mathrm{y}}\left(\beta_{\mathrm{y}}+\mathrm{k}_{1}\right) \cdot \sqrt{1-\beta_{\mathrm{y}}^{2}}}{1+\mathrm{k}_{6} \cdot\left(\beta_{\mathrm{y}}+\mathrm{k}_{1}\right)} \mathrm{d} \beta_{\mathrm{y}}+
$$$$
\int_{\beta_{2}}^{\beta_{3}} \frac{\beta_{y}\left(\beta_{y}+k_{1}\right)^{2} \cdot \sqrt{1-\beta_{y}^{2}}}{1+k_{6} \cdot\left(\beta_{y}+k_{1}\right)} d \beta_{y}+
$$

$$
\int_{\beta_{\mathrm{ei}}}^{\beta_{2}^{\prime}} \frac{\beta_{\mathrm{y}} \sqrt{1-\beta_{\mathrm{y}}^{2}}}{\mathrm{k}_{11} \cdot\left(\beta_{\mathrm{y}}+\mathrm{k}_{1}\right)^{2}+\mathrm{k}_{12}\left(\beta_{\mathrm{y}}+\mathrm{k}_{1}\right)} d \beta_{\mathrm{y}}+
$$

$$
\mathrm{k}_{5} \frac{\beta_{\mathrm{y}}\left(\beta_{\mathrm{y}}+\mathrm{k}_{1}\right) \sqrt{1-\beta_{\mathrm{y}}^{2}}}{1+\mathrm{k}_{6} \cdot\left(\beta_{\mathrm{y}}+\mathrm{k}_{1}\right)} \mathrm{d} \beta_{\mathrm{y}}-
$$




$$
\begin{aligned}
& \int_{\beta_{7}^{\prime}}^{\beta^{\prime}{ }_{3}} \frac{\beta_{y}\left(\beta_{y}+k_{1}\right)^{2} \cdot \sqrt{1-\beta_{y}^{2}}}{1+k_{6} \cdot\left(\beta_{y}+k_{1}\right)} d \beta_{y}{ }^{\prime}+ \\
& \omega \text { n } \\
& +-. \Sigma \sigma_{\mathrm{si}} \cdot \beta_{\mathrm{si}} \cdot \eta_{\mathrm{si}} \\
& f_{y d} \quad i=1
\end{aligned}
$$

\subsection{ROTEIRO PARA A ELABORAÇÃO DOS DIAGRAMAS}

Os diagramas momento fletor - força normal - curvatura são feitos de forma iterativa, através dos seguintes passos (FUSCO, 1986):

1) Adota-se um valor para a curvatura adimensional ( $h / r)$.

2) Definem-se os intervalos para variação da profundidade da linha neutra $\left(\beta_{\mathrm{xi}} \mathrm{e}\right.$ $\left.\beta_{\mathrm{xs}}\right)$.

3) Calculam-se as forças normais adimensionais.

4) Verifica-se se a força normal de cálculo está entre o intervalo de valores calculado no passo 3 .

5) Caso não esteja, calcula-se novo valor para a profundidade da linha neutra, interpolando entre os limites.

6) Calcula-se novamente a normal resistente.

7) Verifica-se o equilíbrio entre a normal de cálculo e a normal resistente; assim que este se verificar, passa-se ao passo seguinte, caso contrário, volta-se ao passo 5 .

8) Calcula-se o momento fletor adimensional.

9) Volta-se ao passo 1 com novo valor para $h / r$. 


\section{MÉTODOS PARA OBTENÇÃO DOS MOMENTOS DE SEGUNDA ORDEM}

Os momentos fletores de segunda ordem são decorrentes dos deslocamentos ocorridos no elemento estrutural, devidos ao carregamento a que está sujeito. Utilizando-se a teoria do método geral, estes deslocamentos podem ser obtidos pela integração numérica das curvaturas, ao longo do eixo do elemento, ou através da analogia de Mohr (ver seção 4.2).

A determinação dos momentos fletores totais, no caso de verificação da estabilidade de elementos pré-dimensionados, é feita de forma iterativa pelo método do equilíbrio (seção 5.3). Este método é a base do processo de Engesser-Vianello, onde os deslocamentos são obtidos através da analogia de Mohr.

Serão apresentados, para integração numérica, o método da regra dos trapézios e o da integração do polinômio interpolador de Lagrange.

\subsection{INTEGRAÇÃO NUMÉRICA}

Considere-se uma barra engastada na base e livre no topo, com um carregamento qualquer que produza um diagrama de momentos fletores como o da fig. 7.1. Devido à variação do momento fletor ao longo do eixo da barra, as curvaturas das seções ao longo deste eixo também variam. Para a aplicação do método geral, faz-se necessária a divisão da barra em segmentos, determinando-se as curvaturas em cada extremo dos segmentos para, através da integração destas curvaturas, encontrar as rotações das seções e através da integração das rotações encontrar os deslocamentos transversais da barra. 


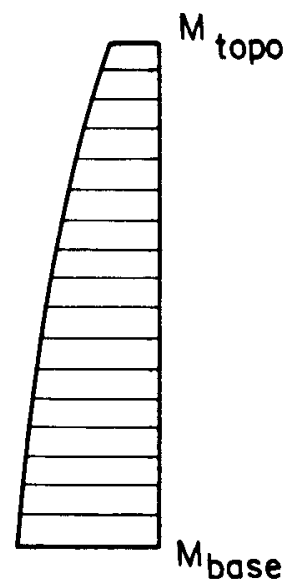

Fig. 7.1 - Diagrama de momentos fletores.

O número de segmentos a serem utilizados depende do método de integração e da precisão desejada.

\subsubsection{REGRA DOS TRAPÉZIOS}

A integral de uma função $f(x)$, em um intervalo [a,b] (fig. 7.2), pode ser aproximada pela área de um trapézio:

$$
\int_{a}^{b} f(x) \cdot d x \cong[f(a)+f(b)]_{2}^{(b-a)} \frac{}{2}
$$

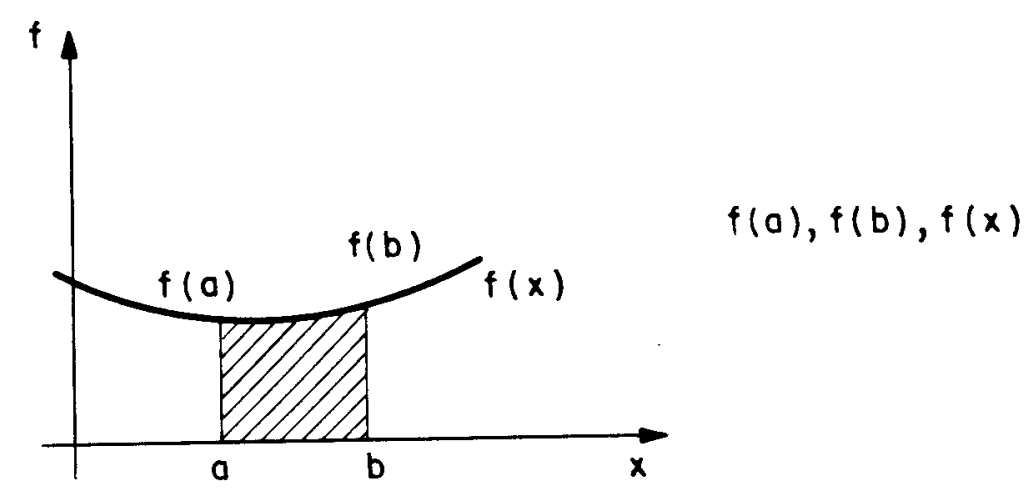

Fig. 7.2 - Diagrama de uma função. 
Subdividindo-se o intervalo $\overline{\mathrm{ab}}$ em vários intervalos de dimensão $\mathrm{h}$, tem-se (HUMES, 1984):

$$
\int_{a}^{b} f(x) \cdot d x \cong \frac{h}{2}\left[f\left(x_{0}\right)+2 f\left(x_{1}\right)+\ldots+2 f\left(x_{n-1}\right)+f\left(x_{n}\right)\right]
$$

Para o pilar genérico da fig. 7.3a, subdividido em segmentos, com as curvaturas das seções (fig. 7.3b) e os deslocamentos transversais (fig. 7.3c), obtémse, para o extremo superior $(k+1)$ de um elemento genérico $K$, a rotação através da integração pela regra dos trapézios:

$$
\left.\frac{\mathrm{dv}}{\mathrm{dx}}\right|_{\mathrm{k}+1} \cong-\frac{\mathrm{h}}{2}\left[(1 / \mathrm{r})_{1}+2(1 / \mathrm{r})_{2}+\ldots+2(1 / \mathrm{r})_{\mathrm{k}}+(1 / \mathrm{r})_{\mathrm{k}+1}\right]+\mathrm{C}_{1}
$$

Integrando-se a expressão 7.3, obtém-se o deslocamento no extremo superior do elemento genérico $\mathrm{K}$ :

$$
\mathrm{v} \mid \mathrm{k}+1 \cong-\left[\left(\frac{\mathrm{d}}{2}\left[\left(\frac{\mathrm{dv}}{\mathrm{dx}}\right)_{1}+2\left(\frac{\mathrm{dv}}{\mathrm{dx}}\right)_{2}+\ldots+2\left(\frac{\mathrm{dv}}{\mathrm{dx}}\right)_{\mathrm{k}}+\left(\frac{\mathrm{dv}}{\mathrm{dx}}\right)_{\mathrm{k}+1}\right]+\mathrm{C}_{1} \mathrm{x}+\mathrm{C}_{2}\right.\right.
$$

As constantes de integração $\left(C_{1}\right.$ e $\left.C_{2}\right)$ são determinadas através das condições de contorno da barra em análise (ver exemplo 7.3.1).

A precisão depende do número de elementos em que é subdividido o pilar, aumentando com o número destes. 


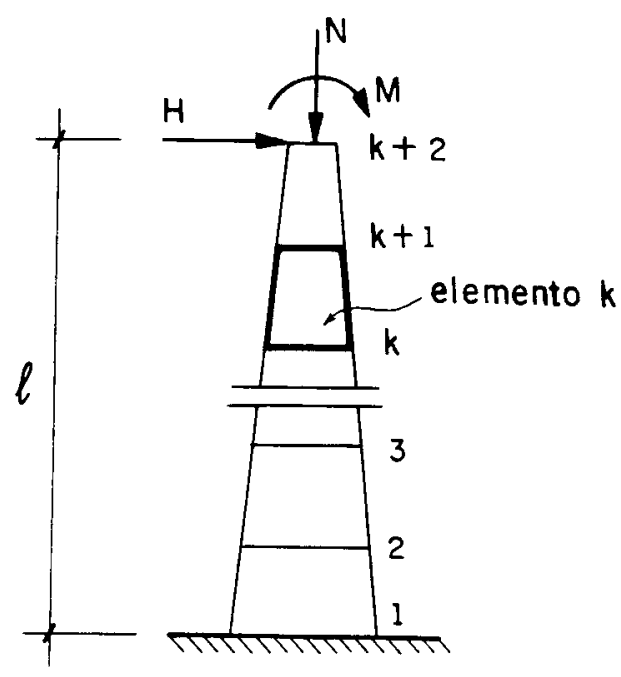

(a)

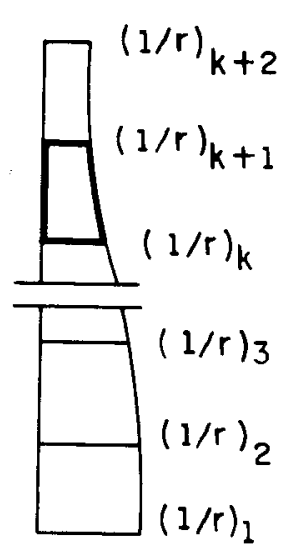

(b)

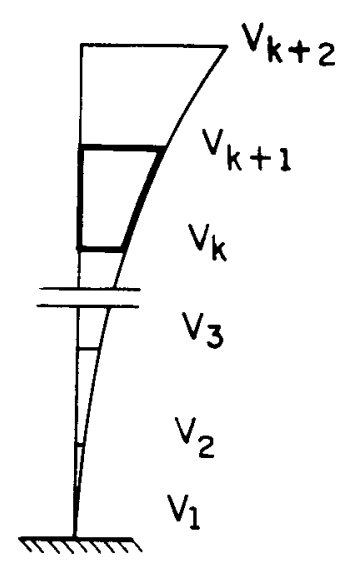

(c)

Fig. 7.3 - Diagramas de curvaturas e deslocamentos.

\subsubsection{INTEGRAÇÃO DO POLINÔMIO INTERPOLADOR DE LAGRANGE}

Considerando-se a função $y=f(x)$ representada na fig. 7.4, esta função pode ser obtida através do polinômio interpolador de Lagrange (MILNE, 1968):

$$
P_{n}(x)=y_{0} A_{0}(x)+y_{1} A_{1}(x)+\ldots+y_{n} A_{n}(x) \cong f(x)
$$

onde:

$$
A_{k}(x)=\frac{\left(x-x_{0}\right)\left(x-x_{1}\right) \ldots\left(x-x_{k-1}\right)\left(x-x_{k+1}\right) \ldots\left(x-x_{n}\right)}{\left(x_{k}-x_{0}\right)\left(x_{k}-x_{1}\right) \ldots\left(x_{k}-x_{k-1}\right)\left(x_{k}-x_{k+1}\right) \ldots\left(x_{k}-x_{n}\right)}
$$

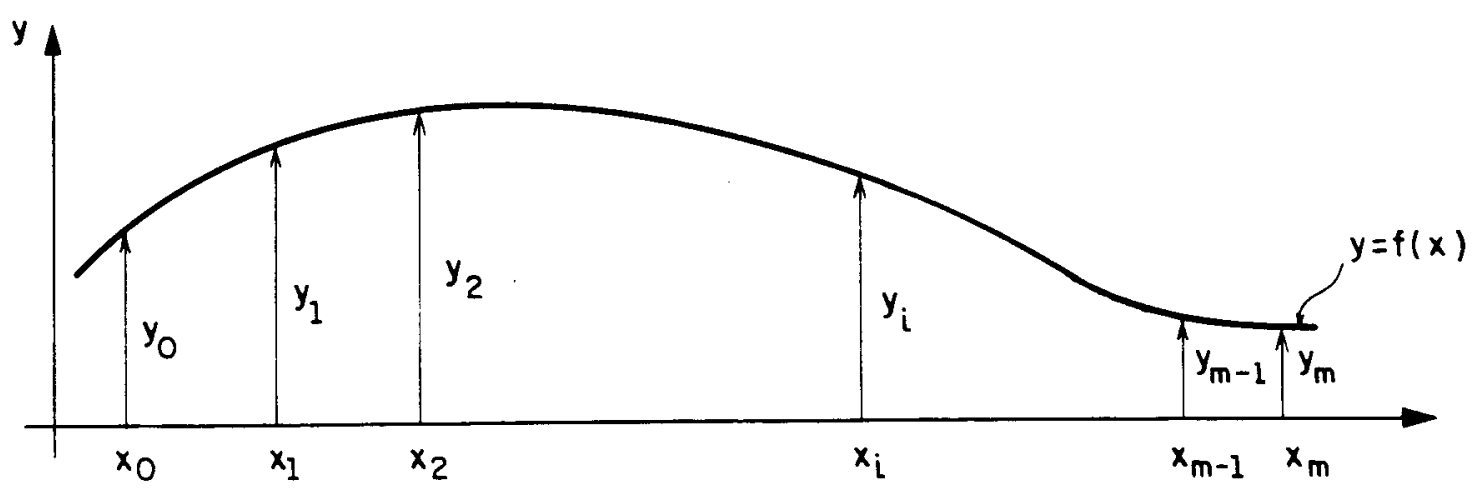

Fig. 7.4 - Diagrama de uma função. 
A expressão 7.5 permite a obtenção dos valores de y em função de $\mathrm{x}$. $\mathrm{O}$ grau do polinômio interpolador é representado por $\mathbf{n}$. No caso da integração pela regra dos trapézios, é utilizado um polinômio interpolador de grau 1 - uma reta (expressão 7.7), aplicado sucessivamente aos pares de ordenadas (curvaturas e rotações).

$$
P_{1}(x)=\frac{\left(x_{1}-x\right) y_{0}+\left(x-x_{0}\right) y_{1}}{\left(x_{1}-x_{0}\right)}
$$

Pode-se obter o valor aproximado da integral de uma função $y=f(x)$ através da integração do polinômio de interpolação determinado pelos pontos dados.

A precisão depende da escolha do grau do polinômio interpolador. Pode-se conhecer $\mathrm{n}$ valores numéricos de uma função cujo polinômio interpolador de grau 2 (parábola do segundo grau) conduza a valores suficientemente precisos. Escolhendo-se um polinômio de grau 3 para a integração das curvaturas ao longo do eixo de um pilar, tendo-se a finalidade de encontrar os deslocamentos transversais, determinam-se três rotações em pontos escolhidos e a partir destas rotações são encontrados os deslocamentos transversais em qualquer ponto deste eixo. Não há necessidade de escolher pontos eqüidistantes como na regra dos trapézios, nem o mesmo número de pontos desejados para o conhecimento dos deslocamentos.

O exemplo 1 mostra os valores dos deslocamentos em um pilar engastado na base e livre no topo, obtidos pela regra dos trapézios e através do polinômio interpolador de Lagrange com grau 2 e 3.

\subsection{ANALOGIA DE MOHR}

O processo é descrito na seção 4.2. O carregamento a ser aplicado na barra conjugada não é obtido diretamente pela razão $\mathrm{M} / \mathrm{EI}$ em cada seção, mas através da utilização das relações momento fletor - força normal - curvatura, já que não há linearidade física nos materiais da barra. A analogia de Mohr é utilizada no método de Engesser-Vianello, destinado à determinação dos momentos solicitantes de segunda ordem. Trata-se de um método iterativo, que consiste em: 
a) Divide-se a barra em n partes iguais;

b) Calculam-se os esforços solicitantes de primeira ordem;

c) Escolhe-se o sistema equivalente de Mohr;

d) Determinam-se os pesos elásticos $\left(\mathrm{W}_{\mathrm{k}}\right)$;

e) Considera-se a barra carregada com cargas concentradas $W_{k}$ nos pontos $k$ e determinam-se os valores de $\overline{\mathrm{M}_{\mathrm{k}}} \mathrm{e} \overline{\mathrm{Q}_{\mathrm{k}}}$;

f) Calculam-se os momentos fletores tendo-se em vista que $\bar{M}$ corresponde ao deslocamento ocorrido sob a ação do carregamento fictício $\mathbf{W}$;

g) Se os momentos fletores calculados no passo f não forem aproximadamente iguais aos momentos fletores da iteração anterior, volta-se ao passo d; caso contrário, os momentos encontrados correspondem aos momentos totais em cada uma das seções em que foi dividida a barra (ver exemplo 7.3.2).

\subsubsection{DETERMINAÇÃO DOS PESOS ELÁSTICOS}

São obtidos admitindo-se uma variação parabólica das curvaturas, determinadas através dos momentos fletores de primeira ordem e das relações $\mathrm{M}-\mathrm{N}$ $1 / r$. Os pesos elásticos são forças fictícias, equivalentes às baricentrais que compõem o diagrama de carregamento da fig. 7.5b, transmitidas para as seções da barra. Os pesos elásticos equivalentes nos pontos isolados $\mathrm{k}$, para o extremo superior da barra, são definidos por (LEONHARDT, 1977):

$$
\mathrm{W}_{0} \cong \frac{\Delta \mathbf{x}}{12}\left[3,5(1 / \mathrm{r})_{0}+3(1 / \mathrm{r})_{1}-0,5(1 / \mathrm{r})_{2}\right]
$$

Para um ponto intermediário $\mathrm{k}$ :

$$
\mathrm{W}_{\mathrm{k}} \cong \frac{\Delta \mathrm{x}}{12}\left[3,5(1 / \mathrm{r})_{\mathrm{k}-1}+3(1 / \mathrm{r})_{\mathrm{k}}-0,5(1 / \mathrm{r})_{\mathrm{k}+1}\right]
$$


Para o extremo inferior da barra:

$$
\mathrm{W}_{\mathrm{n}} \cong \frac{\Delta \mathrm{x}}{12}\left[3,5(1 / \mathrm{r})_{\mathrm{n}}+3(1 / \mathrm{r})_{\mathrm{n}-1}-0,5(1 / \mathrm{r})_{\mathrm{n}-2}\right]
$$

Esta maneira de caracterizar as forças fictícias simplifica o cálculo dos esforços, principalmente na presença de carregamentos parcialmente distribuídos.

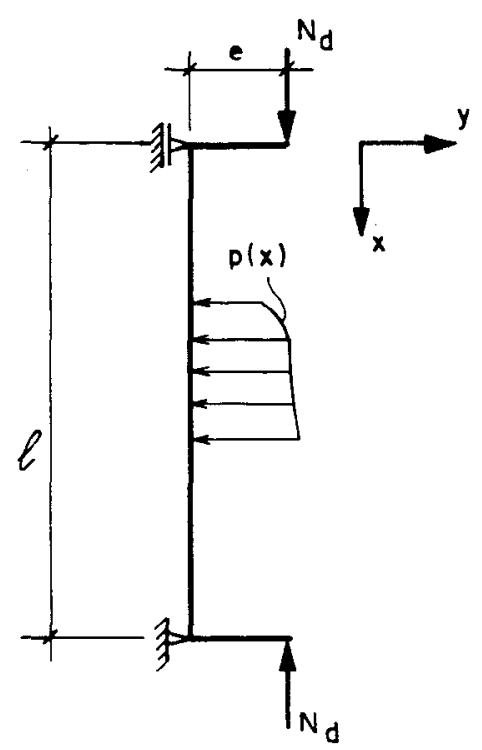

(a)

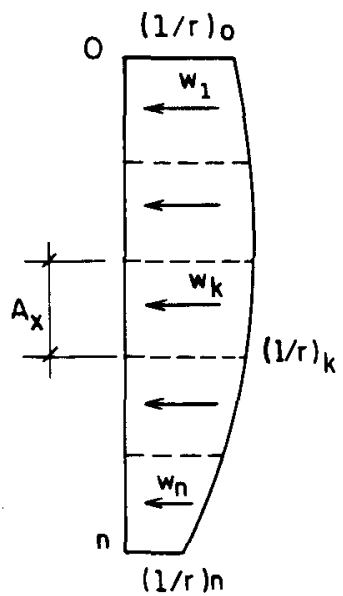

(b)

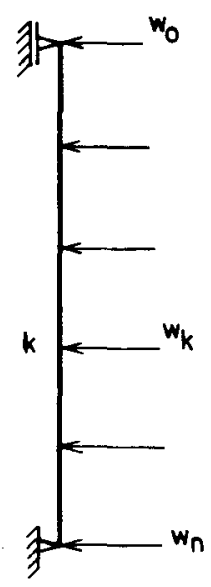

(c)

Fig. 7.5 - Carregamento da barra equivalente.

\subsubsection{REAÇÕES E ESFORÇOS NA BARRA EQUIVALENTE}

As reações de apoio dependem do sistema estático da barra. Para a barra equivalente apoio-engaste (fig. 7.6a), a reação na seção 0 será:

$$
\mathrm{R}_{0}=\sum_{\mathrm{k}=0}^{\mathrm{n}} \mathrm{W}_{\mathrm{k}}
$$

Para as barras equivalentes biapoiadas (fig. 7.6b) ou hipostáticas do tipo da fig. 7.6c: 


$$
\mathrm{R}_{0}=\frac{\sum \mathrm{W}_{\mathrm{k}} \cdot \mathrm{x}_{\mathrm{k}}}{l}
$$

Para a barra hipostática da fig. 7.6c o carregamento é auto-equilibrado, sendo as reações nulas em ambas as extremidades da barra.

Chamando-se o esforço cortante e o momento fletor equivalente de $\overline{\mathrm{Q}}$ e $\overline{\mathrm{M}}$, respectivamente, tem-se para a seção 0 dos casos a, b e c da fig. 7.6:

$$
\begin{aligned}
& \overline{\mathrm{Q}_{0}}=\overline{\mathrm{R}_{0}}-\overline{\mathrm{W}_{0}} \\
& \overline{\mathrm{M}_{0}}=\mathrm{M}_{0}
\end{aligned}
$$

No caso da fig. 7.6d, $\bar{Q}_{0}$ e $\bar{M}_{0}$ são iguais a zero. Para as demais seções, temse:

$$
\begin{aligned}
& {\overline{Q_{k}}}={\overline{Q_{k-1}}}_{-W_{k}} \\
& \overline{\mathbf{M}}_{\mathrm{k}}=\mathrm{Q}_{\mathrm{k}-1} \cdot \Delta \mathrm{x}+\overline{\mathbf{M}}_{\mathrm{k}-1}
\end{aligned}
$$
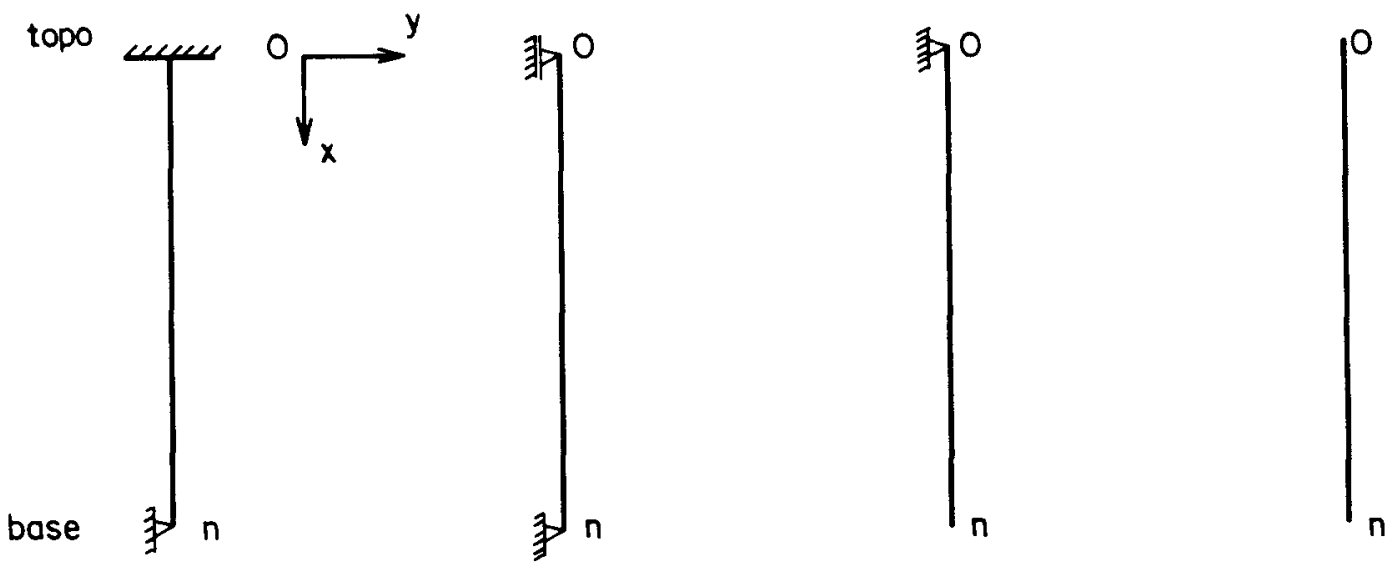

(a)

(b)

(c)

(d)

Fig. 7.6 - Barras equivalentes. 


\subsubsection{NÚMERO DE ITERAÇÕES}

Depende da precisão desejada e da tolerância fixada. Se os momentos fletores da barra equivalente em dada iteração são suficientemente próximos aos valores dos momentos da iteração anterior, pode-se encerrar o processo.

A cada iteração são calculados os pesos elásticos (W), os esforços $(\overline{\mathrm{Q}} \mathrm{e} \overline{\mathrm{M}})$, os momentos totais $\left(\mathrm{M}_{\mathrm{td}}\right)$ e as curvaturas em função destes momentos $(1 / \mathrm{r})$.

A ruína da peça é constatada através das relações $M-N-1 / r$, caso o par de valores de $v$ e $\mu$ conduzir a valores fora da envoltória dos ábacos (ver capítulo 6).

\subsection{EXEMPLOS}

O primeiro exemplo destina-se a uma comparação entre os processos de integração numérica, realizada através da regra dos trapézios e através da integração do polinômio interpolador de Lagrange, com graus 2 e 3 .

O segundo exemplo é um aplicação do método de Engesser-Vianello, para a obtenção dos momentos fletores totais em um pilar de concreto com dimensões e armadura definidas.

7.3.1 Supondo um pilar de seção constante engastado na base e livre no topo (fig. 7.7) que tenha os deslocamentos transversais de seu eixo determinados pela função:

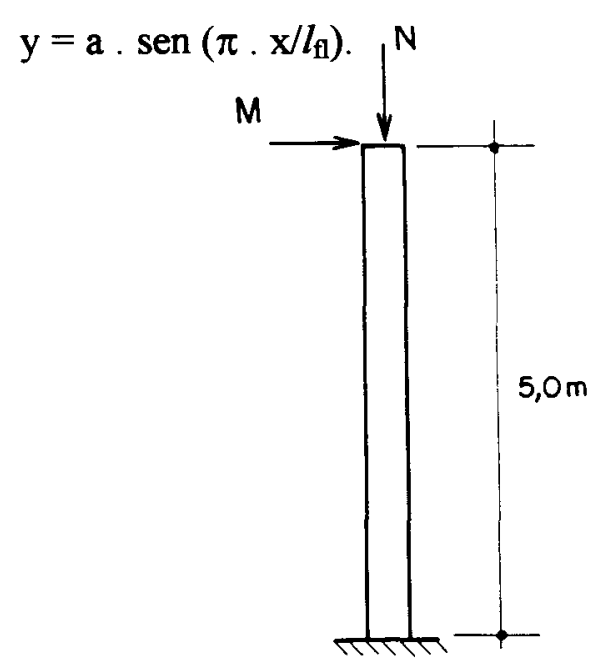

(a)

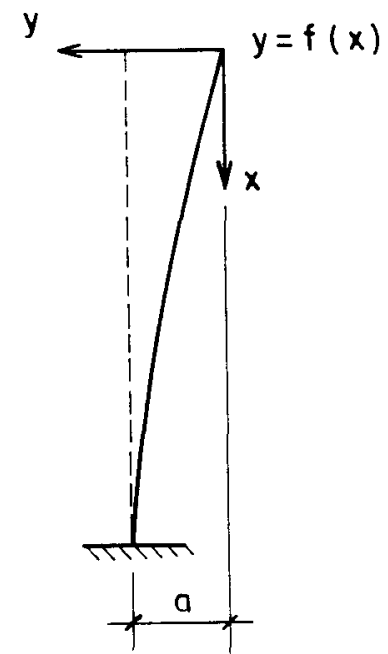

(b)

Fig. 7.7 - Linha elástica de um pilar engastado na base. 
Tem-se para as curvaturas exatas das seções (derivadas segunda em relação a $x$ da função $y)$ :

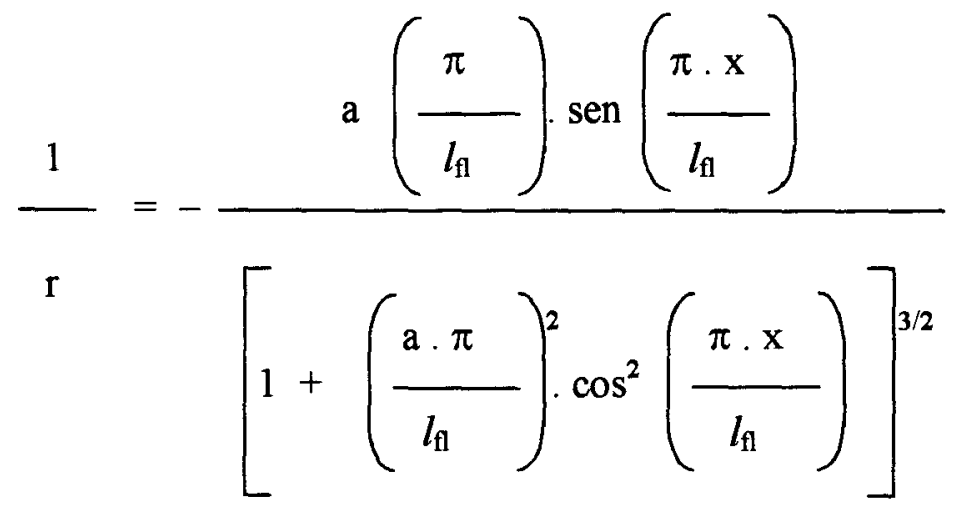

Subdividindo-se o pilar em 10 elementos e adotando-se $a=0,1$, tem-se para as curvaturas em cada seção os valores indicados na tabela 7.1 .

Tabela 7.1 - Valores das curvaturas exatas.

\begin{tabular}{c|c}
\hline $\mathbf{x}(\mathrm{m})$ & $-1 / \mathrm{r}$ (exata) \\
\hline 0 & 0 \\
\hline 0,5 & 0,00154172 \\
\hline 1,0 & 0,00304579 \\
\hline 1,5 & 0,00447545 \\
\hline 2,0 & 0,00579559 \\
\hline 2,5 & 0,00697370 \\
\hline 3,0 & 0,00798196 \\
\hline 3,5 & 0,00879119 \\
\hline 4,0 & 0,00938567 \\
\hline 4,5 & 0,00974774 \\
\hline 5,0 & 0,00986960 \\
\hline
\end{tabular}

a) Determinação das constantes de integração

Das condições de contorno do elemento, tem-se:

- para $x=\ell \rightarrow$ a rotação é nula 


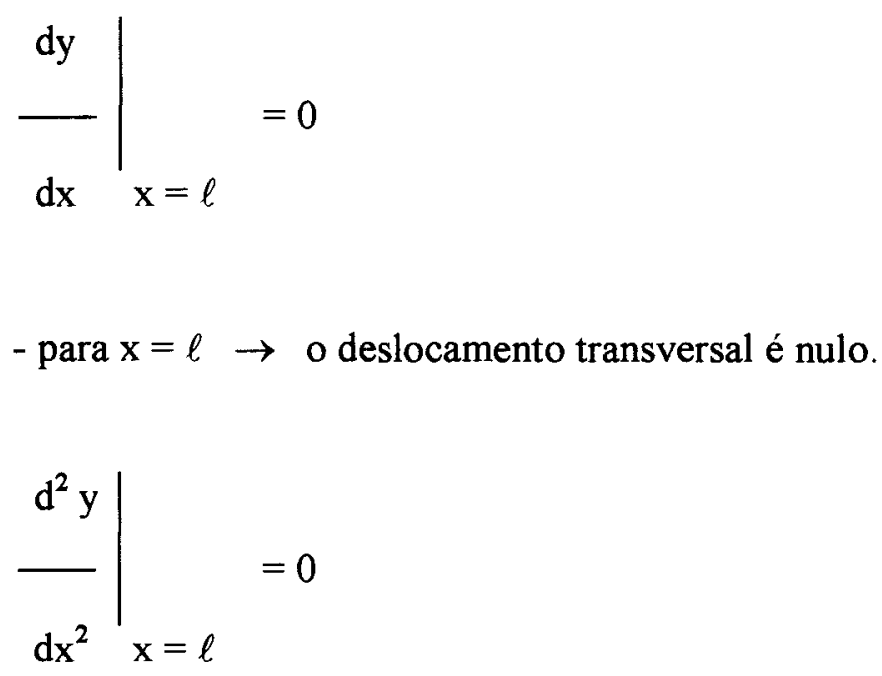

As derivadas primeira e segunda da expressão $y=a \cdot \operatorname{sen}\left(\pi \cdot x / l_{\mathrm{fl}}\right)$ fornecem as rotações e os deslocamentos, respectivamente:
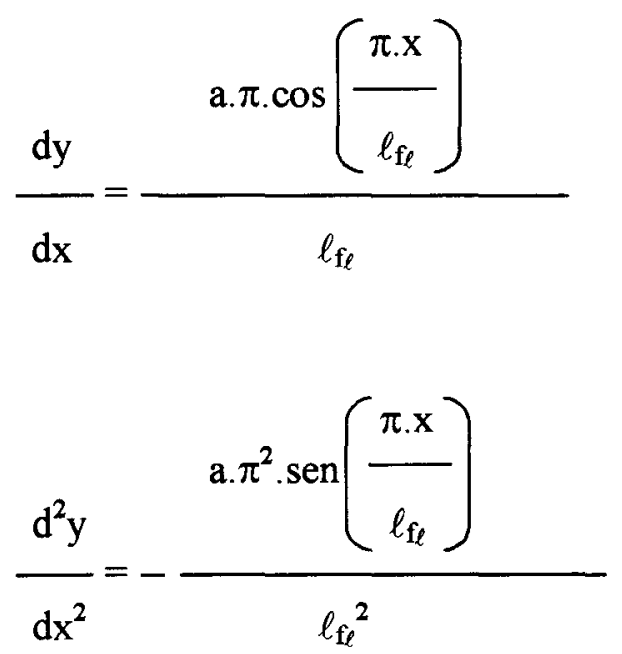

Das condições de contorno anteriores e das derivadas acima, lembando-se que o comprimento de flambagem $\left(\ell_{\mathrm{f}_{\ell}}\right)$ para o pilar em questão é igual a $2 \ell$, tem-se que $\mathrm{C}_{1}=0$ e $\mathrm{C}_{2} \cong \mathrm{a}$.

b) Polinômio interpolador $\operatorname{com} \mathrm{n}=2$.

Utilizando-se a expressão 7.6 para a determinação dos coeficientes $\mathrm{A}$ da expressão 7.5 : 


$$
A_{0}=\frac{\left(x-x_{1}\right) \cdot\left(x-x_{2}\right)}{\left(x_{0}-x_{1}\right)\left(x_{0}-x_{2}\right)}
$$

$$
\begin{aligned}
& A_{1}=\frac{\left(x-x_{0}\right) \cdot\left(x-x_{2}\right)}{\left(x_{1}-x_{0}\right)\left(x_{1}-x_{2}\right)} \\
& A_{2}=\frac{\left(x-x_{0}\right) \cdot\left(x-x_{1}\right)}{\left(x_{2}-x_{0}\right)\left(x_{2}-x_{1}\right)}
\end{aligned}
$$

Integrando-se os coeficientes acima, tendo como limite inferior $b=0$ (origem do eixo $\mathrm{x}$ ) e como limite superior o ponto onde se deseja calcular a rotação ou o deslocamento (valor da abcissa c):

$$
A_{0}=\left[x \frac{2 \cdot x^{2}-3\left(x_{1}+x_{2}\right) x+6 \cdot x_{1} \cdot x_{2}}{6\left(x_{0}-x_{1}\right)\left(x_{0}-x_{2}\right)}\right]
$$

$$
A_{1}=\left[x \frac{2 \cdot x^{2}-3\left(x_{0}+x_{2}\right) x+6 \cdot x_{0} \cdot x_{2}}{6\left(x_{1}-x_{0}\right)\left(x_{1}-x_{2}\right)}\right]
$$

$$
A_{2}=\left[x \frac{2 \cdot x^{2}-3\left(x_{0}+x_{1}\right) x+6 \cdot x_{0} \cdot x_{1}}{6\left(x_{2}-x_{0}\right)\left(x_{2}-x_{1}\right)}\right]
$$


Escolhendo-se arbitrariamente as abcissas $\mathrm{x}_{0}, \mathrm{x}_{1} \mathrm{e} \mathrm{x}_{2}$, calculam-se as correspondentes curvaturas (fórmula da curvatura exata).

$$
\begin{array}{ll}
\mathrm{x}_{0}=0,5 & 1 / \mathrm{r}_{0}=-0,00986960 \\
\mathrm{x}_{1}=2,5 & 1 / \mathrm{r}_{1}=-0,00697370 \\
\mathrm{x}_{2}=5,0 & 1 / \mathrm{r}_{2}=c
\end{array}
$$

Substituindo-se os valores de $\mathrm{x}$, determinando-se os coeficientes e utilizandose a expressão (7.5), obtém-se as rotações:

$$
\begin{array}{ll}
\mathrm{x}_{0}=0,5 & \phi=0,004956347 \\
\mathrm{x}_{1}=2,5 & \phi=0,022093331 \\
\mathrm{x}_{2}=5,0 & \phi=0,031622065
\end{array}
$$

Com estas rotações e as constantes obtidas no item a, determinam-se os deslocamentos nas seções da barra (ver tabela do item e).

c) Polinômio interpolador com $\mathrm{n}=3$.

Utilizando-se a expressão 7.6 para a determinação dos coeficientes $\mathrm{A}$ da expressão 7.5, e integrando-se:

$$
\begin{aligned}
& A_{0}=\left[\int_{b}^{c} \frac{x^{4}}{4}-\frac{x^{3}}{3}\left(x_{1}+x_{2}+x_{3}\right)+\frac{x^{2}}{2}\left[x_{1}\left(x_{2}+x_{3}\right)+x_{2} \cdot x_{3}\right]-\right. \\
& \left.\left.x_{1} \cdot x_{2} \cdot x_{3} \cdot x\right] \cdot\left[\left(x_{0}-x_{1}\right)\left(x_{0}-x_{2}\right)\left(x_{0}-x_{3}\right)\right]-1\right] \\
& A_{1}=\left[\int_{b}^{c} \frac{x^{4}}{4}-\frac{x^{3}}{3}\left(x_{0}+x_{2}+x_{3}\right)+\frac{x^{2}}{2}\left[x_{0}\left(x_{2}+x_{3}\right)+x_{2} \cdot x_{3}\right]-\right.
\end{aligned}
$$




$$
\begin{aligned}
& x_{0} \cdot x_{2} \cdot x_{3} \cdot x \cdot\left[\left(x_{1}-x_{0}\right)\left(x_{1}-x_{2}\right)\left(x_{1}-x_{3}\right)\right]
\end{aligned}
$$

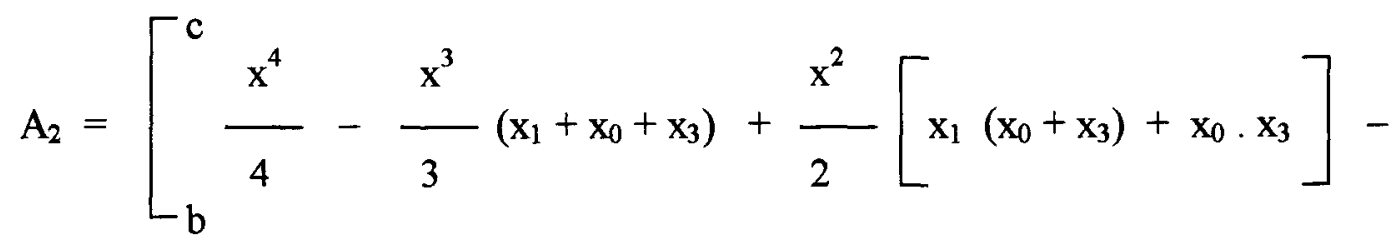

$$
\begin{aligned}
& \left.\left.x_{1} \cdot x_{0} \cdot x_{3} \cdot x\right] \cdot\left[\left(x_{2}-x_{0}\right)\left(x_{2}-x_{1}\right)\left(x_{2}-x_{3}\right)\right]^{-1}\right] \\
& A_{3}=\left[\int_{b}^{c} \frac{x^{4}}{4}-\frac{x^{3}}{3}\left(x_{1}+x_{2}+x_{0}\right)+\frac{x^{2}}{2}\left[x_{1}\left(x_{2}+x_{0}\right)+x_{2} \cdot x_{0}\right]-\right. \\
& \left.\left.\mathrm{x}_{1} \cdot \mathrm{x}_{2} \cdot \mathrm{x}_{0} \cdot \mathrm{x}\right] \cdot\left[\left(\mathrm{x}_{3}-\mathrm{x}_{0}\right)\left(\mathrm{x}_{3}-\mathrm{x}_{1}\right)\left(\mathrm{x}_{3}-\mathrm{x}_{2}\right)\right]\right]
\end{aligned}
$$

Arbitrando-se:

$$
\begin{array}{ll}
\mathrm{x}_{0}=0,5 & 1 / \mathrm{r}_{0}=-0,00986960 \\
\mathrm{x}_{1}=2,0 & 1 / \mathrm{r}_{1}=-0,00798196 \\
\mathrm{x}_{2}=3,5 & 1 / \mathrm{r}_{2}=-0,00447545 \\
\mathrm{x}_{3}=5,0 & 1 / \mathrm{r}_{3}=0
\end{array}
$$

Substituindo-se os valores de $\mathrm{x}$, determinando-se os coeficientes e utilizandose a expressão 7.5 , obtém-se as rotações: 


$$
\begin{array}{ll}
\mathrm{x}_{0}=0,5 & \phi_{0}=0,004903190 \\
\mathrm{x}_{1}=2,0 & \phi_{1}=0,018466293 \\
\mathrm{x}_{2}=3,5 & \phi_{2}=0,027978705 \\
\mathrm{x}_{3}=5,0 & \phi_{3}=0,031408173
\end{array}
$$

Com estas rotações e as constantes obtidas no item a, determinam-se os deslocamentos nas seções da barra (ver tabela do item e).

d) Regra dos trapézios

Utiliza-se a expressão 7.3, determinando-se as rotações em todas as seções da barra. As rotações são empregadas na expressão 7.4 e os deslocamentos transversais são obtidos.

e) Resultados

São apresentados na tabela 7.2 os deslocamentos obtidos para as seções transversais da barra através da expressão 7.7 - y form - da regra dos trapézios - y trap - e da integração do polinômio interpolador de Lagrange com grau 2 e 3 - y Lag $n=2$ e y Lag $n=3$.

Tabela 7.2 - Deslocamentos transversais (em metros).

\begin{tabular}{c|c|c|c|c}
\hline $\mathrm{x}(\mathrm{m})$ & $\mathrm{y}$ form & y trap & $\mathrm{y}$ Lag.n=2 & $\mathrm{y}$ Lag. $\mathbf{n}=\mathbf{3}$ \\
\hline 0 & 0,00000 & 0,00044 & 0,00054 & 0,00001 \\
\hline 0,5 & 0,01564 & 0,01601 & 0,01617 & 0,01566 \\
\hline 1,0 & 0,03090 & 0,03120 & 0,03123 & 0,03090 \\
\hline 1,5 & 0,04540 & 0,04563 & 0,04547 & 0,04543 \\
\hline 2,0 & 0,05878 & 0,05895 & 0,05863 & 0,05880 \\
\hline 2,5 & 0,07071 & 0,07083 & 0,07044 & 0,07072 \\
\hline 3,0 & 0,08090 & 0,08098 & 0,08064 & 0,08090 \\
\hline 3,5 & 0,08910 & 0,08915 & 0,08896 & 0,08911 \\
\hline 4,0 & 0,09510 & 0,09513 & 0,09440 & 0,09513 \\
\hline 4,5 & 0,09877 & 0,09877 & 0,09890 & 0,09880 \\
\hline 5,0 & 0,10000 & 0,10000 & 0,10000 & 0,10000 \\
\hline
\end{tabular}


Os valores médios das diferenças entre os valores dos deslocamentos calculados pela regra dos trapézios e pelo polinômio interpolador de Lagrange (graus 2 e 3) em relação aos valores obtidos pela fórmula foram, respectivamente: $0,49 \%$; $0,73 \%$ e $0,03 \%$.

A precisão dos resultados obtidos através do polinômio interpolador aumenta com o grau do polinômio, havendo ainda a vantagem de não ser necessário o cálculo das rotações em todas as seções para a determinação dos deslocamentos em cada seção (calculam-se as rotações apenas nos pontos que foram escolhidos para a determinação dos coeficientes). O exemplo em questão não apresenta cargas concentradas, cargas parcialmente distribuídas ou momentos aplicados, conduzindo a uma linha elástica sem variações acentuadas em sua deformação, fazendo com que um polinômio de grau 2 ou 3 seja suficientemente preciso. $\mathrm{O}$ grau do polinômio deve ser determinado de acordo com o carregamento, escolhendo-se para as seções de obtenção dos coeficientes, no mínimo, aquelas onde são aplicadas as cargas concentradas e as seções de início e final das cargas distribuídas, além das seções inicial e final da barra, preferencialmente.

A precisão da regra dos trapézios depende unicamente do número de seções em que a barra é dividida, sendo necessária a determinação das rotações em cada seção para se obter os deslocamentos transversais da barra.

Ambos os métodos de integração tornam-se mais viáveis com o emprego de rotina computacional. Neste trabalho, optou-se pelo emprego da regra dos trapézios, onde a precisão é função da subdivisão da barra, não sendo necessário que o usuário opte pelo grau do polinômio ou que determine as seções de determinação dos coeficientes.

7.3.2 Seja o pilar bi-rotulado de seção variável (fig. 7.8a), com uma taxa mecânica igual a 0,80 e sujeito às forças externas da fig. 7.8b. Supõe-se concreto com fck $=15$ $\mathrm{MPa}$, aço CA-50A e relação d'/d=0,10 (HOFFMANN, 1980). 


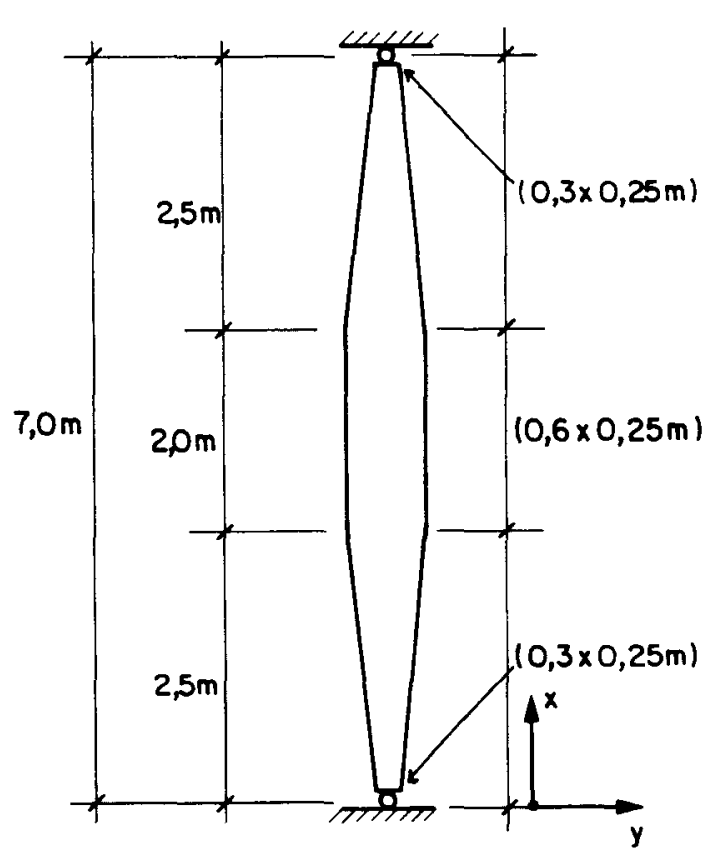

101

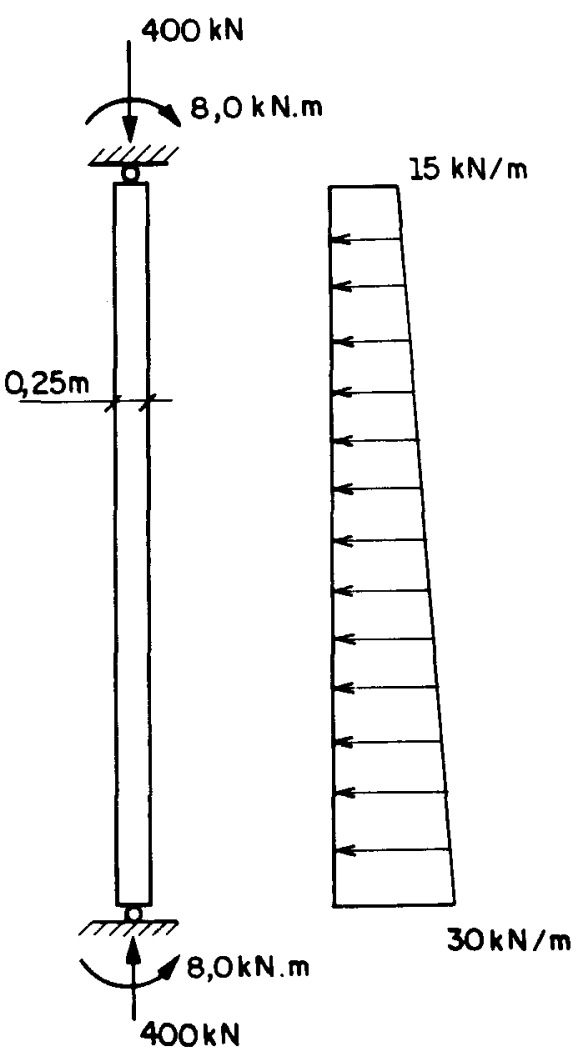

(b)

Fig. 7.8 - Pilar bi-rotulado.

Subdividindo o pilar em dez seções, tem-se:

$\Delta \mathrm{x}=0,70 \mathrm{~m}$

Os momentos fletores de primeira ordem, calculados pela estática, as forças normais e os momentos fletores adimensionais, as curvas relativas (k) e as curvaturas das seções $(1 / r)$ - obtidas do ábaco da fig. 7.9, confeccionado com o diagrama parábola-retângulo para o concreto (HOFFMANN, 1980) - encontram-se na tabela 7.3 . 
Tabela 7.3 - Momento fletores e curvaturas.

\begin{tabular}{c|c|c|c|c|c}
\hline Seção & $\mathbf{M}_{\mathbf{l d}}(\mathrm{kN} \cdot \mathrm{m})$ & $\mu$ & 0 & $-\mathbf{k}$ & $-(1 / \mathrm{r}) \cdot 10^{3}$ \\
\hline 0 & 8,00 & 0,040 & 0,498 & 0,200 & 0,800 \\
\hline 1 & 53,20 & 0,207 & 0,389 & 1,170 & 4,680 \\
\hline 2 & 90,34 & 0,288 & 0,319 & 1,750 & 7,000 \\
\hline 3 & 110,68 & 0,299 & 0,271 & 1,840 & 7,360 \\
\hline 4 & 137,52 & 0,342 & 0,249 & 2,150 & 8,600 \\
\hline 5 & 146,11 & 0,364 & 0,249 & 2,260 & 0,040 \\
\hline 6 & 143,77 & 0,358 & 0,249 & 2,210 & 8,840 \\
\hline 7 & 129,74 & 0,351 & 0,271 & 2,200 & 8,800 \\
\hline 8 & 103,33 & 0,330 & 0,319 & 2,000 & 8,000 \\
\hline 9 & 63,80 & 0,248 & 0,389 & 1,470 & 5,880 \\
\hline 10 & 8,00 & 0,040 & 0,498 & 0,200 & 0,800 \\
\hline
\end{tabular}

As curvaturas (1/r) da tabela 7.3 são as cargas a serem aplicadas na barra equivalente. Determinam-se os pesos elásticos e os esforços na barra equivalente, obtendo os momentos fletores e assim os deslocamentos a cada iteração. A tolerância para os momentos fletores será de $\pm 0,4 \mathrm{kN}$.m.

Os momentos da coluna $M_{t d}$ da tabela relativa à terceira iteração (página 128) correspondem aos momentos totais na barra (primeira e segunda ordem).

O emprego do método de Engesser-Vianello justifica-se quando não se dispõe de suporte computacional para a realização dos cálculos. O método requer a disponibilidade de diagramas momento fletor - força normal - curvatura.

A precisão do método aumenta com o número de seções em que é dividido o elemento, porém, as interpolações efetuadas durante o emprego dos ábacos e a precisão gráfica dos mesmos influem na precisão dos resultados obtidos. 


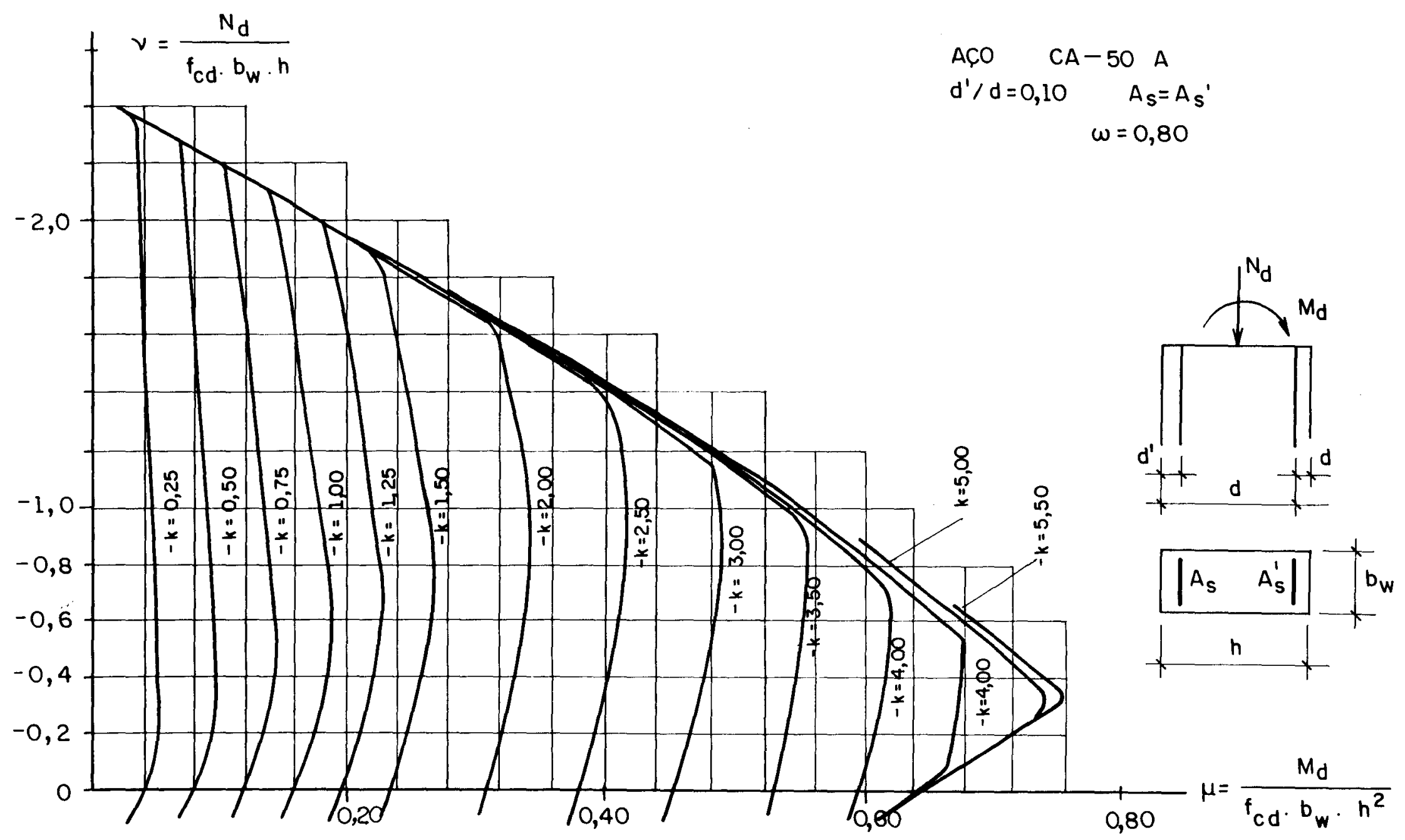

Fig. 7.9 - Diagrama momento fletor- força normal - curvatura para seção retangular simétrica (HOFFMAN, 1980). 
$1^{\underline{a}}$ iteração

\begin{tabular}{c|c|c|c|c|c|c|c|c}
\hline Seç & $\mathbf{x}(\mathbf{m})$ & $\mathbf{W}$ & $\mathbf{W}, \mathbf{x}$ & $\overline{\mathbf{Q}}$ & $\overrightarrow{\mathbf{M}}$ & $\mathrm{y}(\mathbf{m})$ & $\mathbf{M}_{\mathrm{td}}$ & $1 / \mathbf{r . 1 0 ^ { 3 }}$ \\
\hline 0 & 7,00 & 0,778 & 5,446 & 22,880 & 0 & 0 & 8,00 & 0,800 \\
\hline 1 & 6,30 & 3,185 & 20,066 & 19,695 & 16,016 & 0,016 & 59,60 & 5,320 \\
\hline 2 & 5,60 & 4,786 & 26,802 & 14,909 & 29,803 & 0,030 & 102,34 & 8,000 \\
\hline 3 & 4,90 & 5,203 & 25,495 & 9,706 & 40,239 & 0,040 & 126,68 & 8,520 \\
\hline 4 & 4,20 & 5,973 & 25,087 & 3,733 & 47,033 & 0,047 & 156,32 & 9,800 \\
\hline 5 & 3,50 & 6,291 & 22,019 & $-2,558$ & 49,646 & 0,050 & 166,11 & 10,520 \\
\hline 6 & 2,80 & 6,197 & 17,352 & $-8,755$ & 47,856 & 0,048 & 162,97 & 10,280 \\
\hline 7 & 2,10 & 6,116 & 12,844 & $-14,871$ & 41,726 & 0,042 & 146,54 & 10,120 \\
\hline 8 & 1,40 & 5,523 & 7,732 & $-20,394$ & 31,317 & 0,031 & 115,73 & 9,200 \\
\hline 9 & 0,70 & 3,943 & 2,760 & $-24,337$ & 17,042 & 0,017 & 70,60 & 6,520 \\
\hline 10 & 0 & 0,959 & 0 & $-25,296$ & 0 & 0 & 8,00 & 0,800 \\
\hline
\end{tabular}

\section{$2^{\mathrm{a}}$ iteração}

\begin{tabular}{c|c|c|c|c|c|c|c|c}
\hline Sec & $\mathbf{x}(\mathbf{m})$ & $\mathbf{W}$ & $\mathbf{W} \cdot \mathbf{x}$ & $\overline{\mathbf{Q}}$ & $\overline{\mathbf{M}}$ & $\mathbf{y}(\mathbf{m})$ & $\mathbf{M}_{\mathbf{t d}}$ & $1 / \mathbf{r} \cdot 10^{\mathbf{3}}$ \\
\hline 0 & 7,00 & 0,861 & 6,027 & 26,289 & 0 & 0 & 8,00 & 0,800 \\
\hline 1 & 6,30 & 3,617 & 22,787 & 22,672 & 18,402 & 0,018 & 60,40 & 5,520 \\
\hline 2 & 5,60 & 5,474 & 30,654 & 17,198 & 34,273 & 0,034 & 103,94 & 8,160 \\
\hline 3 & 4,90 & 6,008 & 29,439 & 11,190 & 46,311 & 0,046 & 129,08 & 8,840 \\
\hline 4 & 4,20 & 6,827 & 28,673 & 4,363 & 54,144 & 0,054 & 159,12 & 10,000 \\
\hline 5 & 3,50 & 7,308 & 25,578 & $-2,945$ & 57,198 & 0,057 & 168,91 & 10,640 \\
\hline 6 & 2,80 & 7,201 & 20,163 & $-10,146$ & 55,137 & 0,055 & 165,77 & 10,520 \\
\hline 7 & 2,10 & 7,040 & 14,784 & $-17,186$ & 48,035 & 0,048 & 148,94 & 10,160 \\
\hline 8 & 1,40 & 6,337 & 8,872 & $-23,523$ & 36,005 & 0,036 & 117,73 & 9,400 \\
\hline 9 & 0,70 & 4,387 & 3,071 & $-27,910$ & 19,538 & 0,020 & 71,80 & 7,120 \\
\hline 10 & 0 & 1,036 & 0 & $-28,946$ & 0 & 0 & 8,00 & 0,800 \\
\hline
\end{tabular}

$3^{\mathrm{a}}$ iteração

\begin{tabular}{c|c|c|c|c|c|c|c}
\hline Sec & $\mathbf{x}(\mathbf{m})$ & $\mathbf{W}$ & $\mathbf{W} \times$ & $\overline{\mathbf{Q}}$ & $\overline{\mathbf{M}}$ & $\mathbf{y}(\mathbf{m})$ & $\mathbf{M}_{\mathbf{t d}}$ \\
\hline 0 & 7,00 & 0,891 & 6,237 & 26,758 & 0 & 0 & 8,00 \\
\hline 1 & 6,30 & 3,743 & 23,581 & 23,015 & 18,731 & 0,019 & 60,80 \\
\hline 2 & 5,60 & 5,590 & 31,349 & 17,417 & 34,841 & 0,035 & 104,34 \\
\hline 3 & 4,90 & 6,216 & 30,458 & 11,201 & 47,033 & 0,047 & 129,48 \\
\hline 4 & 4,20 & 6,697 & 28,127 & 4,504 & 54,874 & 0,055 & 159,52 \\
\hline 5 & 3,50 & 7,404 & 25,914 & $-2,900$ & 58,027 & 0,058 & 169,31 \\
\hline 6 & 2,80 & 7,350 & 20,580 & $-10,250$ & 55,997 & 0,056 & 166,17 \\
\hline 7 & 2,10 & 7,089 & 14,887 & $-17,339$ & 48,822 & 0,049 & 149,34 \\
\hline 8 & 1,40 & 6,491 & 9,087 & $-23,836$ & 36,685 & 0,037 & 118,13 \\
\hline 9 & 0,70 & 4,748 & 3,324 & $-28,578$ & 19,999 & 0,020 & 71,80 \\
\hline 10 & 0 & 1,135 & 0 & $-29,713$ & 0 & 0 & 8,00 \\
\hline
\end{tabular}




\section{VERIFICAÇÃO DA SEGURANÇA}

Serão abordados, neste capítulo, as ações e os efeitos que devem ser levados em consideração para a verificação da segurança contra os possíveis estados limites, sendo apresentadas as diretrizes sugeridas pela NBR-6118/1978 e pelo CEB-1990.

\subsection{ESTADOS LIMITES}

São estados a partir dos quais o elemento ou a estrutura apresentam desempenho inadequado às finalidades às quais se destina. São divididos em estados limites últimos e estados limites de utilização.

Os estados limites a serem considerados em uma estrutura devem ser especificados pelas normas relativas ao projeto da estrutura.

\subsubsection{ESTADOS LIMITES ÚLTIMOS}

São estados que pela sua simples ocorrência determinam a paralisação, no todo ou em parte, do uso da construção. Usualmente devem ser considerados os estados limites últimos caracterizados por:

a) perda de equilíbrio, global ou parcial, admitida a estrutura como corpo rígido;

b) ruptura ou deformação plástica excessiva dos materiais;

c) transformação da estrutura, no todo ou em parte, em sistema hipostático;

d) instabilidade por deformação;

e) instabilidade dinâmica.

Em casos particulares, pode ser necessário considerar outros estados limites últimos. 


\subsubsection{ESTADOS LIMITES DE UTILIZAÇÃO}

São estados que por sua ocorrência, repetição ou duração causam efeitos estruturais que não respeitam as condições especificadas para o uso normal da construção ou que são indícios de comprometimento da durabilidade da estrutura.

No período de vida da estrutura, usualmente são considerados estados limites de utilização caracterizados por:

a) danos ligeiros ou localizados, que comprometam o aspecto estético da construção ou a durabilidade da estrutura, tais como fissuração ou deformações irreversíveis e microfissuras;

b) deformações excessivas, que afetem a utilização normal da construção ou seu aspecto estético, deformações que dêem origem a danos não aceitáveis nos elementos não estruturais ou que afetem excessivamente a aparência ou a finalidade da estrutura ou dos elementos não estruturais;

c) vibrações de amplitude excessiva, vibrações que resultem em desconforto, apreensão por parte dos usuários ou perda da utilidade da obra.

As vibrações podem ser provocadas por alguns tipos de ações variáveis, tais como:

- movimentos rítmicos provocados por pessoas, tais como: caminhar, pular, correr, dançar;

- ondas causadas pelo vento ou pela água;

- tráfego rodoviário ou ferroviário;

- trabalhos de construção como tráfego pesado, compressão do solo por meio de vibração, cravação de estacas, trabalhos de demolição e explosões.

Os estados limites de utilização decorrem das ações às quais está sujeita a estrutura.

\subsection{AÇÕES}

Ações são as causas que provocam esforços ou deformações nas estruturas. Do ponto de vista prático, as forças e as deformações impostas pelas ações são consideradas como se fossem as próprias ações. 
Os conjuntos das ações, que tem probabilidade não desprezível de atuarem simultaneamente sobre uma estrutura durante um período de tempo pré-estabelecido, especificam os carregamentos da estrutura.

As ações serão combinadas de diferentes maneiras a fim de que seus efeitos mais desfavoráveis possam ser avaliados. As combinações das ações visam a verificação da segurança em relação aos estados limites da estrutura.

\subsubsection{COMBINAÇÕES ÚLTIMAS SEGUNDO A NBR-8641/1984}

Quando se verifica a segurança em relação ao estado limite último são usados coeficientes de ponderação $(\gamma)$, que levam em conta a variabilidade das ações e possíveis erros na avaliação de seus valores, e fatores de combinação e utilização $(\psi)$.

Os valores de $\gamma$ e $\psi$ a serem utilizados nas três combinações apresentadas na seqüência são os indicados nas tabelas 1 a 5 da supracitada norma, salvo indicação em norma específica relativa ao tipo da estrutura em estudo.

\section{A) COMBINAÇÕES ÚLTIMAS NORMAIS}

O carregamento normal decorre do uso previsto para a construção; admite-se que tem duração igual ao período de vida estimado para a estrutura.

$$
\mathrm{F}_{\mathrm{d}}=\sum_{\mathrm{i}=1}^{\mathrm{m}} \gamma_{\mathrm{gi}} \cdot \mathrm{F}_{\mathrm{G}_{\mathrm{i}} \mathrm{K}}+\gamma_{\mathrm{q}}\left[\mathrm{F} \mathrm{Q}_{1} \mathrm{~K}+\sum_{\mathrm{j}=2}^{\mathrm{n}} \psi_{\mathrm{O}_{\mathrm{j}}} \cdot \mathrm{F}_{\mathrm{Q}_{\mathrm{j}} \mathrm{K}}\right]
$$

onde:

$\mathrm{F} \mathrm{G}_{\mathrm{i}} \mathrm{K} \rightarrow$ valor característico das ações permanentes;

$\mathrm{F}_{\mathrm{Q}_{1} \mathrm{~K}} \rightarrow$ valor característico da ação variável, considerada como principal; $\psi_{\mathrm{O}} \cdot \mathrm{F}_{\mathrm{Q}} \mathrm{K} \rightarrow$ valor reduzido das demais ações variáveis.

\section{B) COMBINAÇÕES ÚLTIMAS ESPECIAIS DE CONSTRUÇÃO}

O carregamento decorre da atuação de ações variáveis de natureza ou intensidade especiais. São carregamentos transitórios com duração muito pequena em relação à vida útil da estrutura. 


$$
\mathrm{F}_{\mathrm{d}}=\sum_{\mathrm{i}=1}^{\mathrm{m}} \gamma_{\mathrm{g}_{\mathrm{i}}} \cdot \mathrm{F}_{\mathrm{G}_{1} \mathrm{~K}}+\gamma_{\mathrm{q}}\left[\mathrm{F} \mathrm{Q}_{1} \mathrm{~K}+\sum_{\mathrm{j}=2}^{\mathrm{n}} \psi_{\mathrm{O}_{\mathrm{j}, \mathrm{ef}}} \cdot \mathrm{F} \mathrm{Q}_{\mathrm{j}} \mathrm{K}\right]
$$

onde:

$\psi_{\mathrm{o}_{\mathrm{j}, \mathrm{ef}}} \rightarrow$ fator de combinação para cada uma das demais ações variáveis que possam agir concomitantemente com $\mathrm{F}_{\mathrm{Q}_{1}}$; quando esta tiver um tempo de ação muito pequeno, $\psi_{0}$ pode ser tomado com o correspondente $\psi 2_{j}$.

\section{C) COMBINAÇÕES ÚLTIMAS EXCEPCIONAIS}

Decorrem da atuação de ações excepcionais que podem provocar efeitos catastróficos. $O$ carregamento é transitório, com duração extremamente curta.

$$
\mathrm{F}_{\mathrm{d}}=\sum_{\mathrm{i}=1}^{\mathrm{m}} \gamma_{\mathrm{g}_{\mathrm{i}}} \cdot \mathrm{F}_{\mathrm{G}_{\mathrm{i}} \mathrm{K}}+\mathrm{F} \mathrm{Q}_{\mathrm{exc}}+\gamma_{\mathrm{q}} \cdot \sum_{\mathrm{j}=1}^{\mathrm{n}} \psi_{\mathrm{o}_{\mathrm{j}, \mathrm{ef}}} \cdot \mathrm{F} \mathrm{Q}_{\mathrm{j}} \mathrm{K}
$$

onde:

$\mathrm{F}_{\mathrm{Q}_{\mathrm{exc}}} \rightarrow$ valor da ação transitória excepcional.

\subsubsection{COMBINAÇÕES ÚLTIMAS SEGUNDO O CEB-1990}

Os valores de $\gamma$ e $\psi$ a serem utilizados nas duas combinações previstas neste código são os apresentados nas tabelas 8.1 e 8.2 , respectivamente. São valores de referência, podendo ser substituídos por aqueles indicados em norma específica do CEB, relativa ao tipo de estrutura, ou pelos valores indicados pelas normas vigentes no local onde esta será executada.

\section{A) COMBINAÇÃO FUNDAMENTAL}

Aplicável a situações permanentes e transientes, correspondendo as situações normal e especial da NBR:

$$
\mathrm{F}_{\mathrm{d}}=\sum_{\mathrm{i}=1}^{\mathrm{m}} \gamma_{\mathrm{g}_{\mathrm{i}} \sup } \cdot \mathrm{F}_{\mathrm{G}_{\mathrm{i}} \mathrm{K}_{\text {sup }}}+\sum_{\mathrm{j}=1}^{\mathrm{m}} \gamma_{\mathrm{g}_{\mathrm{i}} \text { inf }} \cdot \mathrm{F}_{\mathrm{G}_{\mathrm{i}} \mathrm{K}_{\mathrm{inf}}}+
$$




$$
+\gamma_{\mathrm{q}}\left[\mathrm{F} \mathrm{Q}_{1} \mathrm{~K}+\sum_{\mathrm{j}=2}^{\mathrm{n}} \psi_{\mathrm{O}_{j}} \cdot \mathrm{F}_{\mathrm{Q}_{\mathrm{j}} \mathrm{K}}\right]
$$

Os índices sup e inf referem-se às parcelas desfavorável e favorável das ações, respectivamente.

\section{B) COMBINAÇÃO ACIDENTAL}

É aplicável a situações acidentais, correspondendo às situações excepcionais da NBR-8681:

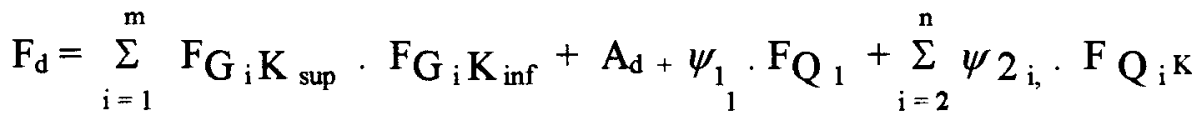

$A_{d}$ é a ação acidental associada com a situação de cálculo; se a ação acidental não for a geradora da combinação ou se esta for o resultado de uma ação passada, $A_{d}=0$.

As ações a serem incluídas em qualquer combinação são aquelas mutuamente compatíveis ou, com aproximação aceitável, que possam ser consideradas como tal. As ações não-simultâneas devem ser consideradas na mesma combinação se seus efeitos forem simultâneos.

Tabela 8.1 - Fatores parciais $\gamma_{\mathrm{F}}-$ Valores Básicos.

\begin{tabular}{cc|c|c}
\hline Ações & Ef. Desfavoráveis & Ef Favoráveis \\
\hline Permanentes & $\gamma_{\mathrm{g}}$ & 1,35 & 1,00 \\
Variáveis & $\gamma_{\mathrm{q}}$ & 1,50 & norm. desprez. \\
Protensão & $\gamma_{\mathrm{p}}$ & 1,10 & 1,00 \\
\hline
\end{tabular}


Tabela 8.2 - Fatores de combinação $\psi$

\begin{tabular}{l|c|c|c}
\hline \multicolumn{1}{c|}{ NATUREZA DAS CARGAS } & $\psi_{0}$ & $\psi_{1}$ & $\psi_{2}$ \\
\hline Edificios residenciais & 0,3 & 0,4 & 0,2 \\
Escritórios e lojas & 0,6 & 0,6 & 0,3 \\
Estacionamentos & 0,6 & 0,7 & 0,6 \\
Vento, neve & 0,5 & 0,2 & 0 \\
\hline
\end{tabular}

\subsubsection{COMBINAÇÕES DE UTILIZAÇÃo}

Consideram-se as ações permanentes, inclusive as deformações impostas permanentes e as ações variáveis, correspondentes a cada combinação, associadas aos fatores de utilização $\left(\psi_{1} \mathrm{e} \psi_{2}\right)$. As três combinações a seguir apresentadas são adotadas pela NBR-8681/1984 e pelo CEB-1990:

\section{A) COMBINAÇÃO QUASE-PERMANENTE DE UTILIZAÇÃO}

São combinações que podem atuar durante grande parte no período de vida da estrutura, da ordem da metade deste período. Todas as ações variáveis são consideradas com seus valores quase-permanentes $\psi_{2}{ }_{\mathrm{j}}, \mathrm{F} \mathrm{Q}_{\mathrm{j}} \mathrm{K}$.

$$
F_{d, \text { uti }}=\sum_{i=1}^{m} F_{G_{i} K}+\sum_{j=1}^{n} \psi_{2} \cdot F Q_{j} K
$$

\section{B) COMBINAÇÕES FREQÜENTES DE UTILIZAÇÃO}

São combinações que se repetem muitas vezes durante o período de vida da estrutura, da ordem de $10^{5}$ vezes em 50 anos, ou que tenham duração total igual a uma parte não desprezivel desse período, da ordem de $5 \%$. A ação variável principal $\mathrm{F}_{\mathrm{Q}_{1}}$ é tomada com seu valor freqüente $\psi_{1} . \mathrm{F}_{\mathrm{QK}_{1}}$ e todas as demais ações variáveis são tomadas com seus valores quase-permanentes $\psi_{2} \cdot \mathrm{F}_{\mathrm{Q}_{\mathrm{j}} \mathrm{K}}$.

$$
\mathrm{F}_{\text {duti }}=\sum_{\mathrm{i}=1}^{\mathrm{m}} \mathrm{F}_{\mathrm{G}_{\mathrm{i}} \mathrm{K}}+\psi_{1} \cdot \mathrm{F}_{\mathrm{Q}_{1} \mathrm{~K}}+\sum_{\mathrm{j}=1}^{\mathrm{n}} \psi_{2_{\mathrm{j}}} \cdot \mathrm{F}_{\mathrm{Q}_{\mathrm{j}} \mathrm{K}}
$$




\section{C) COMBINAÇÕES RARAS DE UTILIZAÇÃO}

São combinações que podem atuar no máximo algumas horas durante o período de vida da estrutura. A ação variável principal $\mathrm{F}_{\mathrm{Q}_{1}}$ é tomada com seu valor característico $\mathrm{F}_{\mathrm{Q}_{1} \mathrm{~K}} \mathrm{e}$ todas as demais ações são tomadas com seus valores freqüentes $\psi_{1} . \mathrm{F}_{\mathrm{Q}_{\mathrm{j}} \mathrm{K}}$ :

$$
\mathrm{F}_{\text {d,uti }}=\sum_{\mathrm{i}=1}^{m} \mathrm{~F}_{\mathrm{G}_{\mathrm{i}} \mathrm{K}}+\mathrm{F}_{\mathrm{Q}_{\mathrm{l}} \mathrm{K}}+\sum_{\mathrm{j}=1}^{\mathrm{n}} \psi_{\mathbf{1}_{\mathrm{j}}} \cdot \mathrm{F}_{\mathrm{Q}_{\mathrm{j}} \mathrm{K}}
$$

\subsection{RESISTÊNCIA DE CÁLCULO}

De forma genérica a resistência de cálculo $f_{d}$ é dada por:

$$
f_{d}=\frac{f_{k}}{\gamma_{m}}
$$

onde:

$\mathrm{f}_{\mathrm{k}}=$ resistência característica;

$\gamma_{m}=$ coeficientes de ponderação ou de minoração das resistências.

\subsubsection{VALORES DA NBR-6118/1978}

De forma geral, utiliza-se para o concreto e para o aço, respectivamente, $\gamma_{\mathrm{c}}=1,4$ e $\gamma_{\mathrm{s}}=1,15$. No cálculo das peças em cuja execução sejam previstas condições desfavoráveis (más condições de transporte, adensamento manual, concretagem deficiente pela concentração de armadura), $\gamma_{\mathrm{c}}$ deve ser elevado para 1,5 .

Para peças pré-moldadas em usina, executadas com cuidados rigorosos, $\gamma_{\mathrm{c}}$ pode ser reduzido para 1,3 . 
Os coeficientes de minoração serão multiplicados por 1,2 quando a peça estiver exposta a ação prejudicial de agentes externos, tais como ácidos, álcalis, águas agressivas, óleos e gases nocivos, temperatura muito elevada ou muito baixa.

\subsubsection{CEB-1990}

Os valores a serem utilizados são apresentados na tabela 7.3 e variam de acordo com a combinação das ações e com o estado limite último considerado (para o estado limite último de flambagem - verificação das deformações $-\gamma_{\mathrm{c}}$ pode ser minorado para 1,2 no caso de combinações fundamentais e 1,0 no caso de combinações acidentais).

Tabela 8.3 - Coeficiente de minoração - CEB-1990.

\begin{tabular}{|c|c|c|}
\hline Var Básica & \multicolumn{2}{|c|}{ Situação de Projeto } \\
\hline Fundamental & Persistentes e Transientes & Acidental \\
\hline \multicolumn{3}{|c|}{ Concreto } \\
\hline Tensão de Comp. & 1,5 & 1,2 \\
\hline Tensão de Tração & \multicolumn{2}{|c|}{ Valores especificos } \\
\hline \multicolumn{3}{|c|}{ Aço comum ou de Protensão } \\
\hline Tensão de Tração & 1,15 & 1,0 \\
\hline Tensão de Comp. & 1,15 & 1,0 \\
\hline
\end{tabular}

\subsection{FLEXÃO OBLÍQUA}

Em alguns casos é possível simplificar a verificação da segurança efetuandose a redução da flexão oblíqua em duas flexões normais (seção 3.3) ou outro processo equivalente. Estas simplificações acarretam em imprecisões que podem ser contra ou a favor da segurança (PINHEIRO, 1994).

As situações em que são admitidas tais simplificações são apresentadas a seguir. 


\subsubsection{NBR-6118/1978}

Quando o pilar em estudo for de seção retangular e possuir armadura igual nas quatro faces permite-se substituir a flexo-compressão oblíqua por uma flexocompressão normal equivalente, em uma direção principal, com excentricidade $\left(e_{x}+\beta \cdot e_{y} \cdot h / b\right)$ em que $e_{x}$ e $e_{y}$ são as projeções de e sobre os eixos principais, considera-se a direção $\mathrm{x}$ aquela em que $\mathrm{e}_{\mathrm{x}} \geq \mathrm{e}_{\mathrm{y}}$. h/b (fig. 8.1). Os valores de $\beta$ são dados em tabela no item 5.1.1.3 desta norma em função da taxa de armadura da seção e da força normal adimensional.

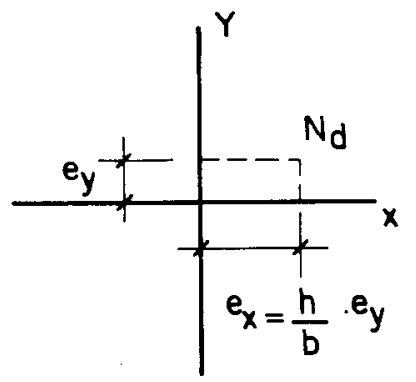

Situaçāo para o cólculo

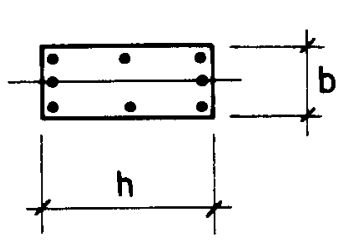

Situaçāo equivolente

Fig. 8.1 - Substituição da flexo-compressão oblíqua.

\subsubsection{CEB-1990}

Para elementos de seção retangular a decomposição da flexão pode ser realizada em dois planos principais, se a força normal $\mathrm{N}$ está localizada próxima de um do eixos principais de inércia, correspondendo às áreas hachuradas da figura 8.2. As relações das excentricidades correspondentes $e_{x 1} / b$ e $e_{y 1} / h$ devem satisfazer uma das seguintes condições:

$$
\begin{aligned}
& \left(e_{y 1} / h\right) /\left(e_{x 1} / b\right) \leq 1 / 4 \\
& \left(e_{x 1} / b\right) /\left(e_{y 1} / h\right) \leq 1 / 4
\end{aligned}
$$

As excentricidades $e_{\mathrm{x} 1}$ e $e_{\mathrm{y} 1}$ são aquelas consideradas segundo as direções de b e $h$, respectivamente, e incluem, além da excentricidade de aplicação da força normal, a consideração das imperfeições geométricas (ver seção 8.6). 


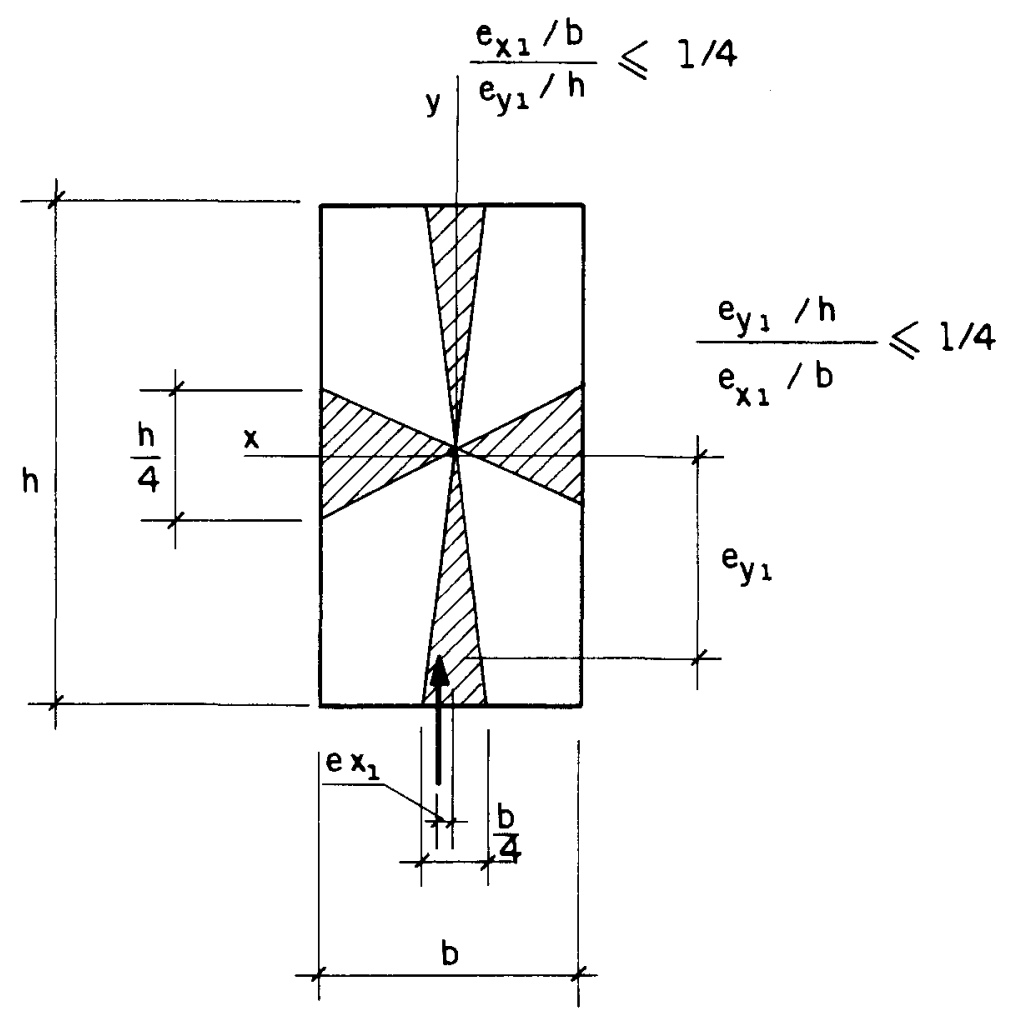

Fig. 8.2 - Condições para a decomposição da força oblíqua.

\subsection{DEFORMAÇÕES}

A necessidade de consideração dos efeitos de $2^{2}$ ordem, seus métodos de avaliação e limites de aplicação são abordados neste item. O processo aproximado citado nos sub-ítens seguintes, comuns à NBR e ao CEB, é o método do pilar padrão e/ou pilar padrão melhorado (FUSCO, 1986 e PAULA, 1988), não apresentados neste trabalho.

\subsubsection{NBR-6118/1978}

O cálculo com consideração das deformações, que abrange tanto o caso de ruína por ruptura a compressão do concreto como o de ruína por instabilidade, será feito:

- Pelo processo exato, obrigatoriamente quando $\lambda \geq 140$, que considera a relação momento-curvatura, baseada nos diagramas tensão-deformação do concreto e do aço; a força normal de cálculo será determinada com: 


$$
\gamma_{\mathrm{f}}=1,4+0,01(\lambda-140)
$$

- Por processo aproximado, devidamente justificado, se $80>\lambda \leq 140$;

- Por processo simplificado, se $40<\lambda \leq 80$, através de acréscimo do momento fletor de $1^{\mathrm{a}}$ ordem de um momento complementar $\mathrm{M}_{2 \mathrm{~d}}$, agindo em plano paralelo à excentricidade acidental com que se calculou o momento de primeira ordem, sendo válido para peças retas e de seção constante, inclusive armadura e força normal.

$$
\begin{aligned}
& \mathbf{M}_{2 \mathrm{~d}}=\mathrm{N}_{\mathrm{d}} \mathrm{e}_{2}=\mathrm{N}_{\mathrm{d}} \cdot \frac{l_{e}^{2}}{10} \cdot \frac{1}{\mathrm{r}} \\
& \frac{1}{\mathrm{r}}=\frac{0,0035+\mathrm{f}_{\mathrm{yd}} / \mathrm{E}_{\mathrm{s}}}{(v+0,5) \mathrm{h}} \operatorname{com} v+0,5 \geq 1
\end{aligned}
$$

onde:

$$
\begin{aligned}
& v=\frac{\mathrm{F}_{\mathrm{d}}}{\mathrm{A}_{\mathrm{c}} \cdot \mathrm{f}_{\mathrm{cd}}} \\
& \mathrm{h}=\text { lado, paralelo à excentricidade acidental considerada, do retângulo } \\
& \text { circunscrito à seção. }
\end{aligned}
$$

- O cálculo com consideração das deformações pode ser desprezado caso $\lambda \leq 40$.

\subsubsection{CEB-1990}

Elementos isolados tanto podem ser elementos únicos sujeitos a compressão ou parte integrante de uma estrutura, na qual podem ser considerados isolados para efeito de projeto. 
Métodos simplificados ou aproximados não devem levar a diferenças maiores que $10 \%$, na capacidade de resistência à flexão do elemento considerado ou da estrutura como um todo, maiores que $10 \%$, na direção da menor resistência, daquelas obtidas com uma análise mais rigorosa de $2^{\underline{a}}$ ordem.

Os efeitos de $2^{\mathrm{a}}$ ordem, para colunas isoladas, podem ser negligenciados se o índice de esbeltez $(\lambda)$ da coluna satisfaz o seguinte critério:

$$
\lambda \leq \lambda_{1}
$$

onde $\lambda_{1}$ é uma esbeltez limite apropriada que leva em conta o decréscimo da capacidade de carga devido à consideração dos efeitos de $2^{2}$ ordem.

Os limites da esbeltez são sempre considerados afetados pela taxa de armadura. Os valores abaixo são os menores limites e são válidos para a armadura mínima. $\mathrm{Na}$ ausência de uma análise mais rigorosa, o limite de esbeltez $\lambda_{1}$ pode ser tomado como:

Elementos deslocáveis:

$$
\begin{array}{ll}
\lambda_{1}=\frac{7,5}{\sqrt{v_{\text {sd }}}} & \text { se } v_{\text {sd }} \leq 0,39 \\
\lambda_{1}=12 \quad \text { se } v_{\text {sd }}>0,39
\end{array}
$$

Elementos indeslocáveis:

$$
\begin{aligned}
& \lambda_{1}=\frac{7,5\left(2-\mathrm{e}_{01} / \mathrm{e}_{02}\right)}{\sqrt{v_{\text {sd }}}} \quad \text { se } v_{\text {sd }} \leq 0,39 \\
& \lambda_{1}=12\left(2-\mathrm{e}_{01} / \mathrm{e}_{02}\right) \quad \text { se } v_{\text {sd }}>0,39
\end{aligned}
$$

onde: 
$\mathrm{e}_{01}$ : menor valor da excentricidade do efeito da carga axial em uma das extremidades do elemento considerado;

$\mathrm{e}_{02}$ : maior valor da excentricidade do efeito da carga axial em uma das extremidades do elemento considerado;

$v_{\text {sd: }}$ força normal adimensional.

Um elemento é considerado indeslocável quando a influência dos deslocamentos sofridos por este elemento, quando da ação de cargas, podem ser desprezados.

\subsection{IMPERFEIÇÕES GEOMÉTRICAS}

Os efeitos de $1^{\underline{a}}$ ordem devidos às imperfeições precisam sempre ser considerados para colunas ou outros membros e estruturas, para os quais estas imperfeições são explicitamente definidas. A não consideração dos efeitos destas imperfeições pode resultar em uma maior descontinuidade entre a capacidade de resistir à flexão entre os efeitos de $1^{\mathrm{a}}$ e $2^{\mathrm{a}}$ ordem.

Estes efeitos podem ser considerados de diferentes maneiras, dependendo do tipo de elemento em estudo (pórticos, elementos isolados).

Para elementos isolados, segundo o CEB-1990, os efeitos das imperfeições geométricas podem ser considerados pelo aumento da excentricidade da força normal de projeto $\left(e_{2}\right)$ na direção mais desfavorável:

$$
\mathrm{e}_{\alpha}=\alpha_{\alpha} \cdot \frac{l}{2}
$$

onde:

$$
\alpha_{\alpha}=\frac{1}{100 \sqrt{l}} \leq \frac{1}{200}
$$

$l=$ comprimento do elemento (metros). 
A consideração destas imperfeições pela NBR-61118/1978 é tomada como igual a $\mathrm{h} / 30$, não sendo menor que $2 \mathrm{~cm}$, sendo $\mathrm{h}$ a maior dimensão da seção na direção em que se considera a excentricidade.

\subsection{FLUÊNCIA}

Os efeitos da fluência e da retração do concreto são normalmente avaliados separadamente e de forma independente. Na realidade a fluência é significativamente maior se for acompanhada pela retração (GLBERT, 1988).

A figura 8.3 mostra o desenvolvimento gradual da deformação de fluência em função do tempo. No período imediatamente posterior a aplicação do primeiro carregamento $\left(\mathrm{t}_{\tau}\right)$, o aumento da deformação é muito rápido, porém depois de vários meses a taxa de aumento decresce sensivelmente, tornando-se muito pequena após vários anos. É usual admitir que a fluência assume um valor limite quando o tempo se aproxima do infinito.

A magnitude da fluência e sua taxa de desenvolvimento são afetadas por vários fatores, alguns são relativos às propriedades do concreto e outros dependem das condições ambientais e de carregamento. $\mathrm{O}$ aumento da resistência, da qualidade, da quantidade e do diâmetro máximo dos agregados reduz a fluência, que também decresce com a idade do concreto.

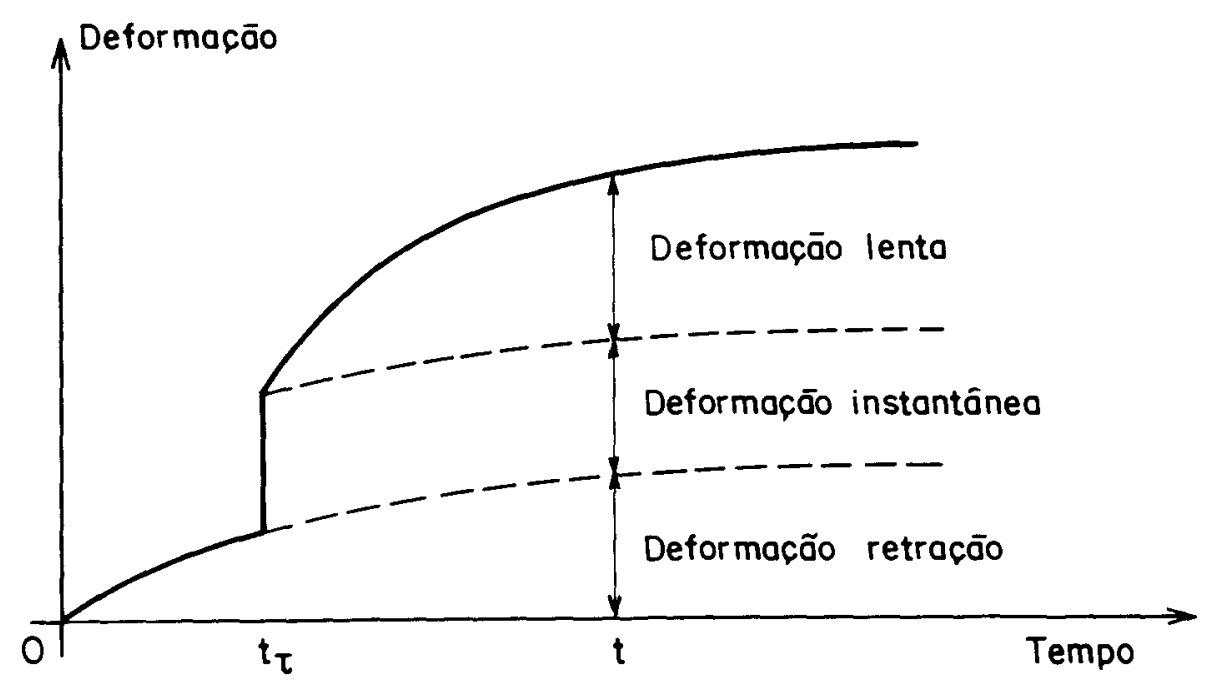

Fig. 8.3 - Deformações do concreto sob tensão constante. 
A fluência depende do nível de tensão a que está sujeito o concreto. Enquanto as tensões permanentes forem menores que a metade da resistência a compressão do concreto, a fluência é aproximadamente proporcional ao nivel das tensões e pode-se empregar a teoria linear da fluência. Sob tensões maiores a fluência aumenta em taxas mais elevadas, devido ao aumento das microfissuras no concreto, e assume uma relação não-linear, conforme mostra a figura 8.4 .

As tensões de compressão raramente excedem a metade da tensão admissível a compressão do concreto em elementos submetidos às cargas de utilização.

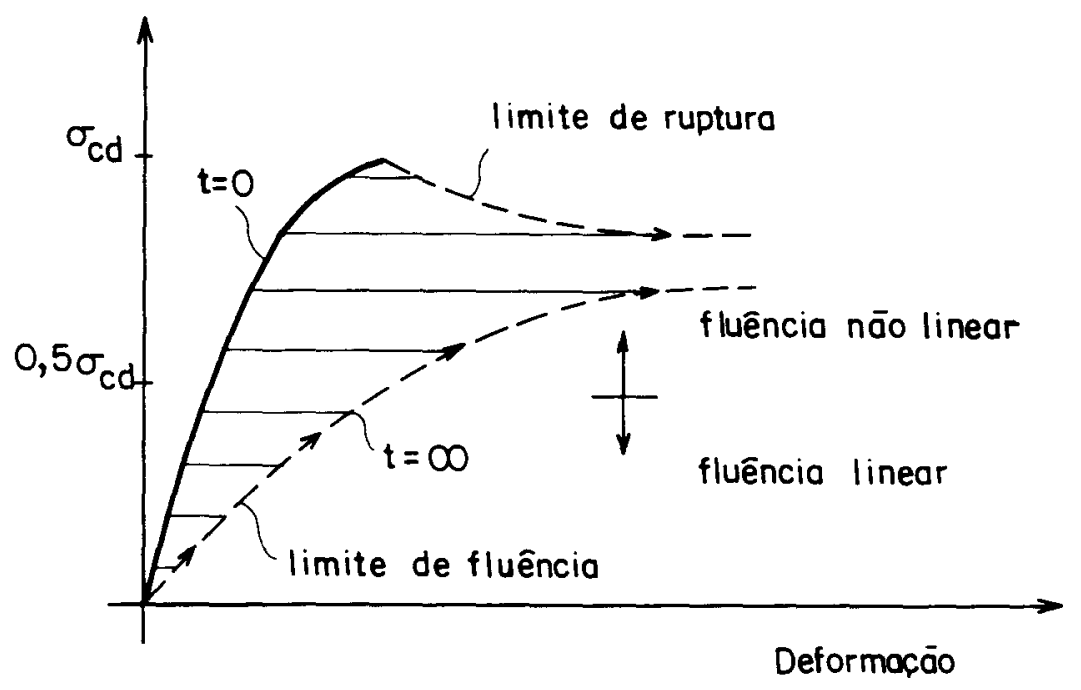

Fig. 8.4 - Influência da intensidade e duração do carregamento nas deformações do concreto.

\subsubsection{NBR-6118/1978}

Quando for necessário levar em conta a fluência e a retração do concreto na determinação dos esforços solicitantes, sua consideração poderá ser feita conforme a NBR-7179 - PROJETO DE ESTRUTURAS DE CONCRETO PROTENDIDO onde são oferecidas diretrizes para a determinação do coeficiente de fluência $\phi$, que é a somatória dos coeficientes de fluência rápida, (ocorre nas primeiras 24 horas após a aplicação da carga que a originou), coeficiente de deformação lenta reversível e coeficiente de deformações lenta irreversível.

A deformação lenta será levada em conta se $\lambda>80$ e quando o elemento estiver sujeito a cargas de longa duração. 
Em FUSCO-1986 são apresentados dois métodos, semelhantes aos sugeridos pelo CEB, para a consideração dos efeitos da fluência.

\section{A) FUNÇÃO EQUIVALENTE DE FLUÊNCIA}

O cálculo é realizado como se toda a carga fosse de longa duração, adotandose para a função equivalente de fluência o valor efetivo:

$$
\phi_{\mathrm{ef}}=\alpha \cdot \beta \cdot \phi\left(\mathrm{t}_{0} \cdot \mathrm{t}_{\infty}\right)
$$

onde:

$\alpha=$ fração da força normal que produz fluência;

$\beta=$ fração do momento fletor de $1^{a}$. ordem que produz fluência;

$\phi\left(t_{0} \cdot t_{\infty}\right)=$ função de fluência real do problema.

$\mathrm{O}$ coeficiente equivalente de fluência $\left(\phi_{\mathrm{ef}}\right)$ será utilizado para a determinação do diagrama $\sigma-\varepsilon$ do concreto (fig. 8.5), a ser utilizado na obtenção dos diagramas $M, N, 1 / r$ (seção 6).

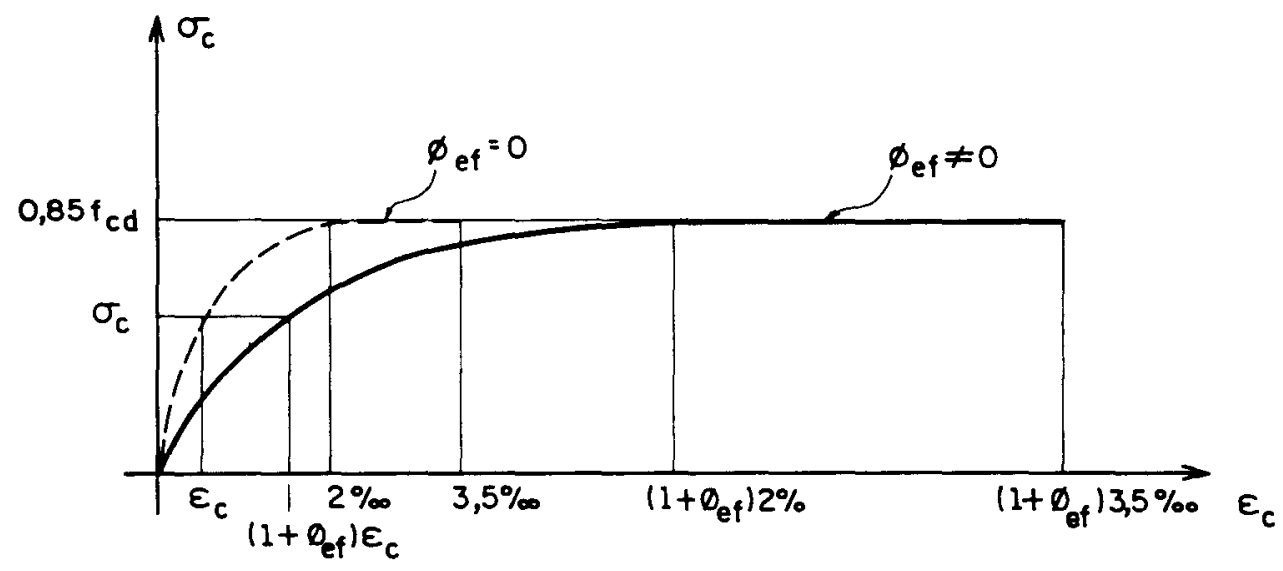

Fig. 8.5 - Diagrama $\sigma-\varepsilon$ do concreto sob o efeito da fluência.

\section{B) MÉTODO DA EXCENTRICIDADE EQUIVALENTE}

Este método admite que todo o carregamento seja de curta duração, introduzindo-se para todas as forças longitudinais uma excentricidade suplementar $\left(e_{c}\right)$ de $1^{a}$. ordem, dada por: 


$$
e_{c}=e_{1 g}\left\{\exp \left(\frac{\phi_{(t o, t \infty)} \cdot N_{g}}{N_{E} / N_{g}}\right)-1\right\}
$$

onde:

$\mathrm{N}_{\mathrm{g}}=$ carga de longa duração que produz fluência;

$\mathrm{N}_{\mathrm{E}}=10 \cdot \mathrm{E}_{\mathrm{c}} \cdot \mathrm{I}_{\mathrm{c}} / l_{\mathrm{e}}^{2}$ carga de flambagem de Euler, com $\mathrm{E}_{\mathrm{c}}$ (módulo de deformação longitudinal do concreto) calculado de acordo com a NBR-6118/1978;

$\mathrm{e}_{\mathrm{g}}=$ excentricidade de $1^{\underline{a}}$ ordem, na qual se inclui a excentricidade acidental.

\subsubsection{CEB-1990}

Os efeitos da fluência podem ser desprezados quando pelo menos duas das seguintes condições forem satisfeitas simultaneamente:

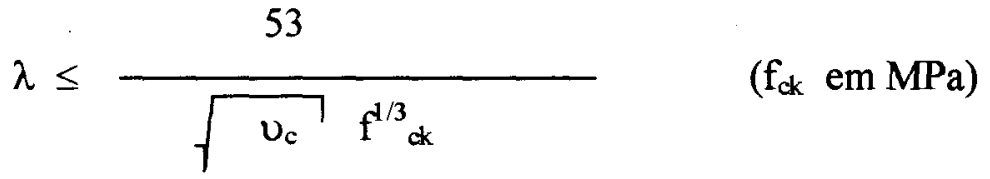

$\mathrm{e}_{0} \geq 2 \mathrm{~h}$

$\alpha \cdot \beta \leq 0,2$

sendo:

$$
v=\frac{N_{s g}}{f_{c k} \cdot A_{c}}
$$

onde:

$\mathrm{N}_{\mathrm{sg}}=$ carga axial sobre as ações quase-permanentes;

$\mathrm{e}_{\mathrm{o}}=$ excentricidade inicial de $\mathrm{N}$;

$\mathrm{h}=$ altura da seção transversal;

$\alpha=$ relação entre a força normal (combinação quase-permanente) $\mathrm{N}_{\mathrm{sg}}$ e a força total de projeto $\mathrm{N}_{\mathrm{sd}}$, considerada no estado limite último; 
$\beta=$ relação entre o momento fletor de projeto obtido com a combinação quasepermanente $\left(\mathrm{M}_{\mathrm{sg}}\right)$ e o momento fletor total considerado no estado limite último $\left(\mathrm{M}_{\mathrm{sd}}\right)$.

Uma das duas simplificações seguintes pode ser usada para considerar os efeitos aproximados da fluência:

- O elemento é calculado diretamente para o estado limite último, aplicando a combinação das ações mais desfavorável e incluindo os efeitos das imperfeições geométricas, utilizando uma relação tensão-deformação modificada, que é obtida pelo aumento de todas as deformações com o fator:

$$
\mathrm{k}_{3}=1+\alpha \cdot \beta
$$

- Os efeitos da fluência também podem ser introduzidos nos cálculos como uma excentricidade adicional $\mathrm{e}_{\mathrm{c}}$ :

$$
e_{c}=\left(e_{o}+e_{a}\right) \cdot\left\{\exp \left(\frac{\phi}{N_{E} / N_{s g}-1}\right)-1\right\}
$$

onde:

$\mathrm{N}_{\mathrm{sg}}=$ força axial permanente no elemento;

$N_{\mathrm{E}}=E_{\mathrm{cm}} \cdot \mathrm{I}_{\mathrm{c}} \cdot\left(\pi / l_{0}\right)^{2}$ carga crítica de Euler para o elemento, $\mathrm{I}_{\mathrm{c}}$ é o momento de inércia da seção de concreto não-fissurado; se a fissuração é passível de ocorrer sob ações permanentes, uma apropriada redução de $\mathrm{I}_{c}$ é conveniente;

$\mathrm{e}_{\mathrm{c}}=$ é a excentricidade adicional remanescente devida aos efeitos da fluência, quando o elemento é considerado hipoteticamente descarregado:

$\mathrm{e}_{0}=$ excentricidade inicial;

$\mathrm{e}_{\mathrm{a}}=$ excentricidade acidental proveniente de imperfeições geométricas;

$\phi=$ coeficiente de fluência. 


\section{ROTINA COMPUTACIONAL}

Apresenta-se neste capítulo rotina informatizada de cálculo, elaborada em linguagem PASCAL para microcomputador, destina da a verificação da estabilidade de pilares de concreto de seção variável, elaborada com base no método geral. Apresenta-se também os resultados obtidos na verificação de um pilar de seção retangular, mostrando os resultados obtidos com a adoção das diretrizes da Norma Brasileira NBR-6118/1978 e do Código Modelo do CEB-1990.

\subsection{EXEMPLO DE APLICAÇĀO}

A verificação do pilar da fig. 9.1 foi efetuada através do programa da seção 9.2, utilizando-se a opção de seguir as recomendações da NBR-6118/1978 e a opção de seguir as recomendações do CEB-1990. Este exemplo foi extraído de HOFFMAN (1980), onde encontra-se verificado pelo método exato, através do processo de Engesser-Vianello (utilizando a analogia de Mohr e ábacos momento fletor - força normal - curvatura).

Dados complementares:

- concreto: $\mathrm{f}_{\mathrm{ck}}=18 \mathrm{MPa}$;

- aço: CA-50A;

- cobrimento: $10 \mathrm{~cm}$;

- área de aço na base: $0,0085 \mathrm{~m}^{2}(\omega=0,4)$, barras dispostas junto às faces horizontais da seção.

O coeficiente de minoração da resistência do concreto $\left(\gamma_{c}\right)$ utilizado na verificação segundo a NBR-6118/1978 foi de 1,4, utilizando-se o diagrama parábola- 
retângulo para as relações tensão-deformação do concreto; para a verificação segundo o CEB-1990, foi utilizado coeficiente de minoração 1,2 e o respectivo diagrama para as relações tensão-deformação do concreto, encontrando-se na tabela 9.1 os resultados obtidos para os deslocamentos e os momentos finais.

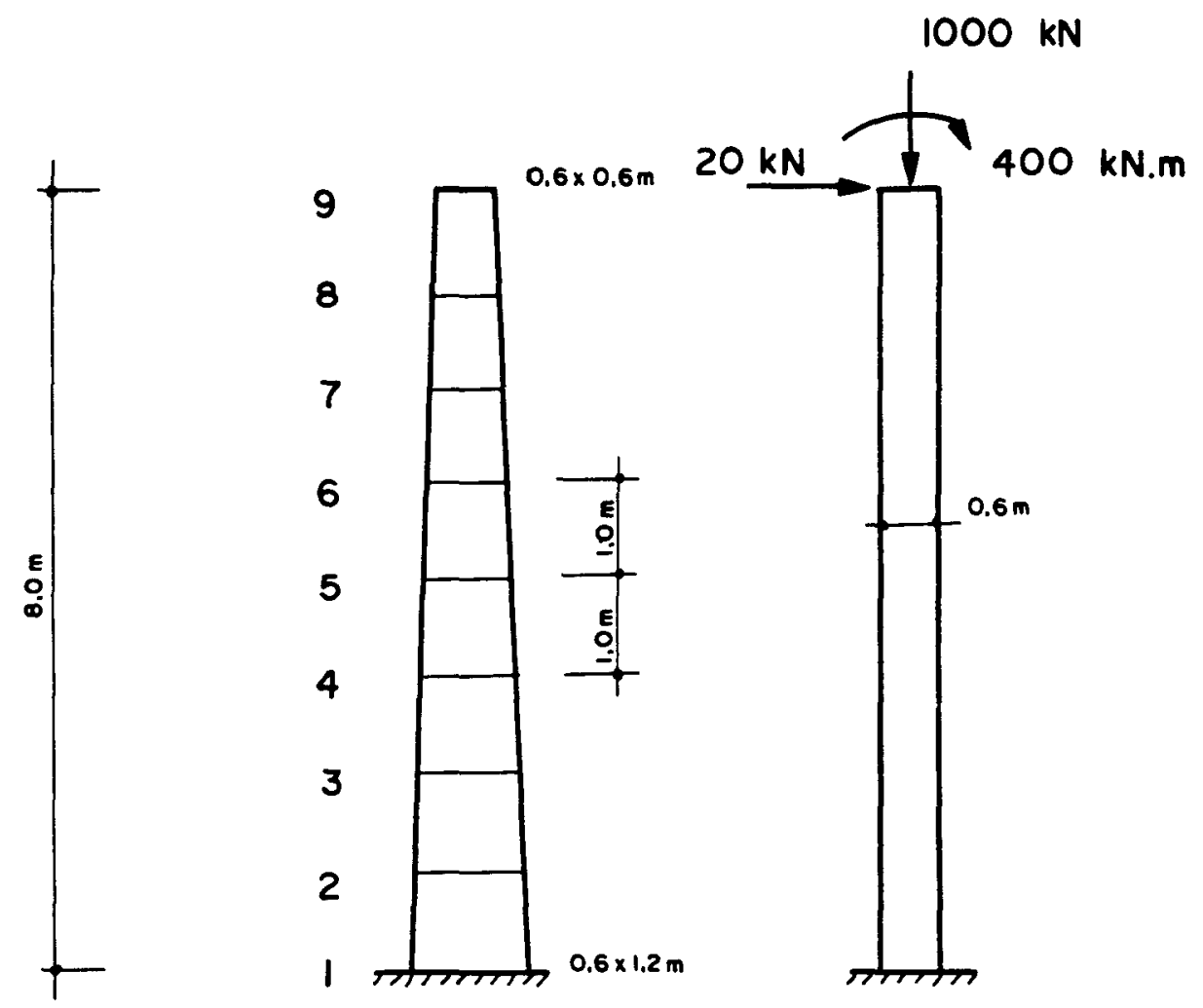

Fig. 9.1 - Pilar livre-engastado com seção transversal variável.

Tabela 9.1 - Valores dos deslocamentos e momentos finais.

\begin{tabular}{c|c|c|c|c|c|c|c}
\cline { 2 - 8 } \multicolumn{2}{c}{} & \multicolumn{2}{c|}{ ABNT } & \multicolumn{2}{c|}{ HOFFMAN } & \multicolumn{2}{c}{ CEB } \\
\hline Seção & Mom. Inic. & Desloc. & Mom. Fin. & Desloc. & Mom. Fin. & Desloc. & Mom. Fin. \\
\hline 1 & 560 & 0,0 & 691 & 0,0 & 671 & 0,0 & 642 \\
\hline 2 & 540 & 0,026 & 669 & 0,031 & 649 & 0,022 & 620 \\
\hline 3 & 520 & 0,049 & 643 & 0,058 & 624 & 0,037 & 597 \\
\hline 4 & 500 & 0,068 & 613 & 0,080 & 595 & 0,051 & 570 \\
\hline 5 & 480 & 0,076 & 579 & 0,090 & 556 & 0,058 & 538 \\
\hline 6 & 460 & 0,095 & 540 & 0,113 & 528 & 0,070 & 511 \\
\hline 7 & 440 & 0,104 & 498 & 0,123 & 489 & 0,077 & 477 \\
\hline 8 & 420 & 0,109 & 451 & 0,129 & 446 & 0,080 & 442 \\
\hline 9 & 400 & 0,111 & 400 & 0,131 & 400 & 0,082 & 400 \\
\hline
\end{tabular}

Momentos em kN.m e deslocamentos em metros. 
Os resultados de Hoffman foram obtidos pelo método de Engesser-Vianello, com o emprego de ábacos. A diferença entre os valores dos momentos fletores finais obtidos por Hoffman e aqueles obtidos pelo programa, segundo os critérios da $\mathrm{ABNT}$, varia de $1,1 \%$ a $4,1 \%$, tendo como variação média $2,6 \%$. As diferenças observadas são decorrentes das interpolações necessárias à utilização dos diagramas momento fletor - força normal - curvatura empregadospelo supracitado autor e às tolerâncias adotadas no programa desenvolvido para a verificação do equilíbrio da forças normais e dos momentos fletores solicitantes e resistentes, além da tolerância das curvaturas das seções.

Os valores dos máximos momentos fletores finais (seção 1) obtidos segundo os critérios do CEB e da ABNT diferem em 4,3\%, sendo esta diferença decorrente dos critérios constantes no código modelo e na norma.

\subsection{PROGRAMA}

Destina-se à verificação da estabilidade de pilares de concreto de seção variável, utilizando a teoria do método geral. $\mathrm{O}$ programa foi desenvolvido para pilares de seção retangular ou circular, cheias ou vazadas, oferecendo opções de cálculo utilizando as relações de tensão-deformação do concreto segundo o diagrama parábola-retângulo (NBR-6118/1978) ou pelo diagrama proposto pelo CEB-1990.

A entrada de dados pode ser feita via teclado, para a primeira inserção de um pilar (um arquivo com os dados inseridos é gerado) ou via arquivo de dados, caso haja alterações no pilar calculado, fazendo-se as alterações diretamente no arquivo de dados gerado pelo programa quando o pilar foi calculado pela primeira vez.

PROGRAM PILARES;

uses Printer;

TYPE PAL30=STRING[30];

TYPE VET10I=ARRAY [1..10] OF INTEGER;

TYPE VET150I=ARRAY [1..150] OF INTEGER;

TYPE VET5R=ARRAY[1..5] OF REAL;

TYPE VET10R=ARRAY [1..10] OF REAL;

TYPE VET150R=ARRAY [1..150] OF REAL;

TYPE MAT1050=ARRAY $[1 . .10,1 . .50]$ OF INTEGER; 
VAR OP,OPS,NSP,i,j,k,l,NTS,NSC,N,TOLINTER,OPPROP,NSD:INTEGER;

TA:CHAR;

FCK,FYK,EC,EY,INCURV,TOLNOR,TOLMOM,TOLCURV,GAMAY,GAMAC,PES:REAL; $\mathrm{NE}$ :VET10I;

Q,HI,HF,TCD:VET5R;

ES,ALS,ESTO,ARACOS,BA,BATO,AL,ALTO,DIA,DIATO:VET10R;

VD,HD,MD,NP,BASE,ALTUR,ESPES,SSEC,TAXARM,DIAM,ALSEC,VOL:VET150R;

ARS:TEXT;

NOME:pal30;

NCA: VET150I;

HACO,ALCO:MAT1050;

PROCEDURE FAZ (var ops,nsp,nts,nsc,tolinter,opprop:INTEGER; var ta:CHAR;

var fck,fyk,ec, ey, incurv, tolnor, tolmom, tolcurv, pes, gamac,gamay:REAL; var ne:VET10I;

var es,esto,als,aracos,ba,bato,al,alto,dia, diato:VET10R; var vd,hd,md:VET150R);

VAR ARQS:TEXT;

j,N:INTEGER;

NOME,TITULO:PAL30;

BEGIN

WRITE ('NOME DO ARQUTVO PARA A GRAVACAO DOS DADOS = ');

READLN (NOME);

WRITE ('IDENTIFICACAO DO PILAR = ');

READLN (TITULO);

ASSIGN (ARQS,NOME);REWRITE(ARQS);

WRITELN (ARQS,TITULO);

WRITELN ('FORMA DO PILAR [1] RETANGULAR CHEIO

[2] RETANGULAR

VAZADO')

WRITE (' [3] CIRCULAR CHEIO [4] CIRCULAR VAZADO ');

READLN (OPS); WRITELN (ARQS,OPS);

WRITE ('NUMERO DE SEGMENTOS DO PILAR = ');

READLN (NSP);

WRITE ('NUMERO TOTAL DE SECOES = ');

READLN (NTS); WRITELN(ARQS,NSP, ' ',NTS);

FOR $i:=1$ TO NTS DO

BEGIN

ES[i]: $=0$;

ESTO[i]: $=0$;

$\mathrm{BA}[\mathrm{i}]:=0$;

BATO[i]: $=0$;

AL[i]: $=0$;

ALTO[i]: $=0$;

DIA $[\mathrm{i}]:=0$;

DIATO[i]: $=0$;

$\mathrm{VD}[\mathrm{i}]:=0$;

$\mathrm{HD}[\mathrm{i}]:=0$;

$\mathrm{MD}[\mathrm{i}]:=0$;

END;

FOR $i:=1$ TO NSP DO

BEGIN

WRITELN ('SEGMENTO',i);

WRTTE (' No DE ELEMENTOS DO SEGMENTO = ');

READLN (NE[i]);WRITELN(ARQS,'SEGMENTO',i);

WRITELN (ARQS,NE[i]);

WRITE (' ALTURA DO SEGMENTO = ');

READLN (ALS[i]);WRITELN(ARQS,ALS[i]); 


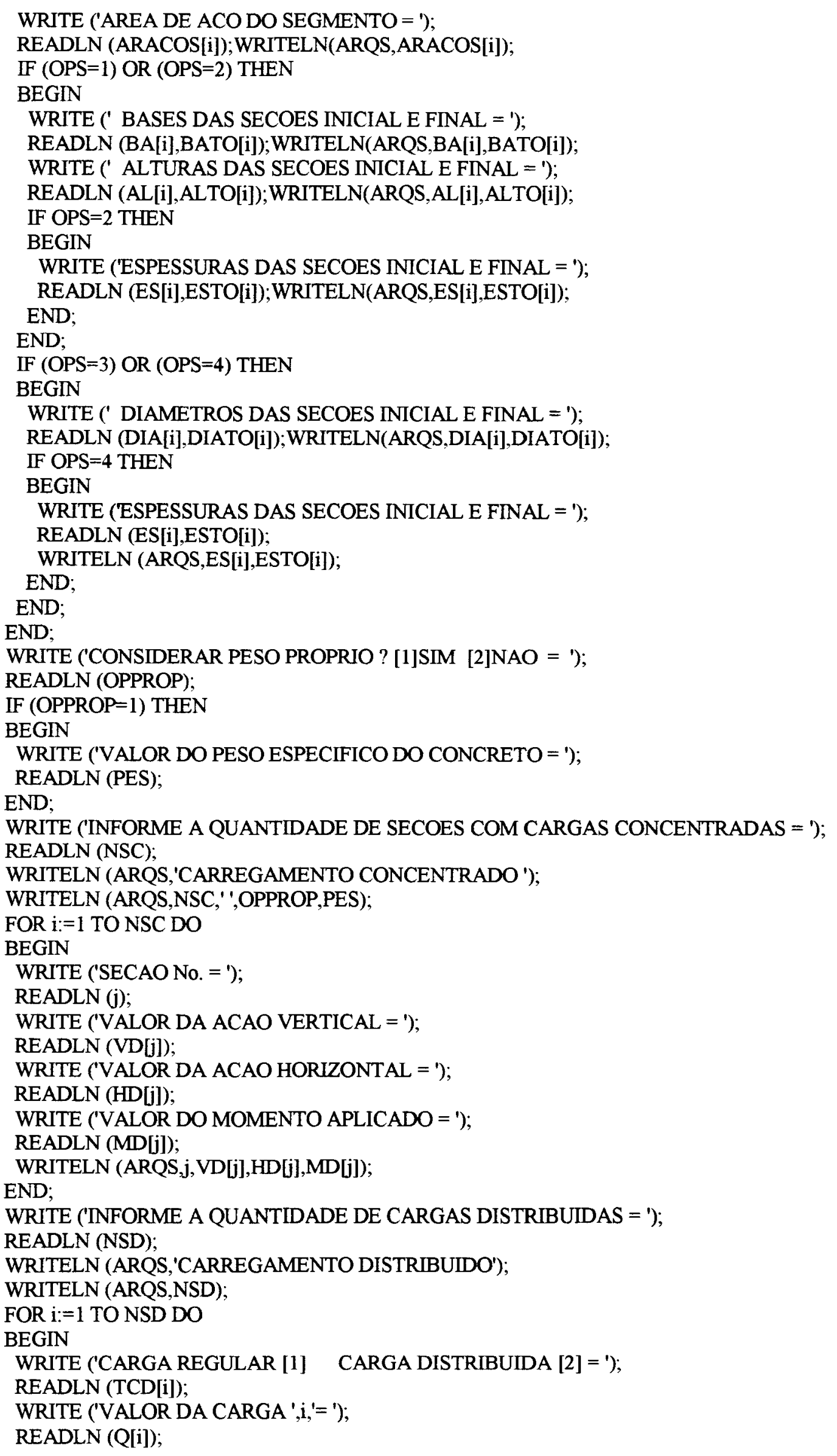




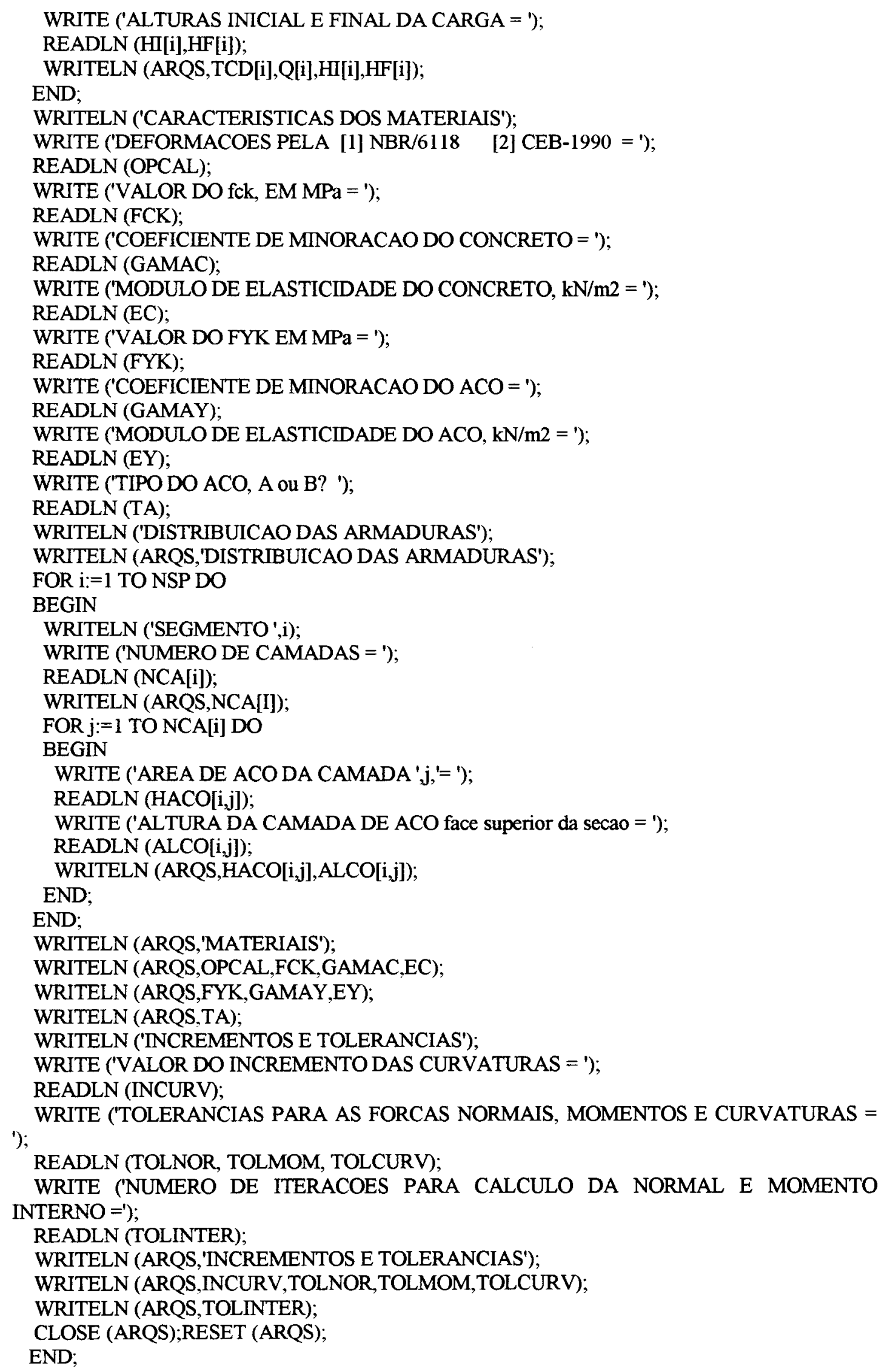




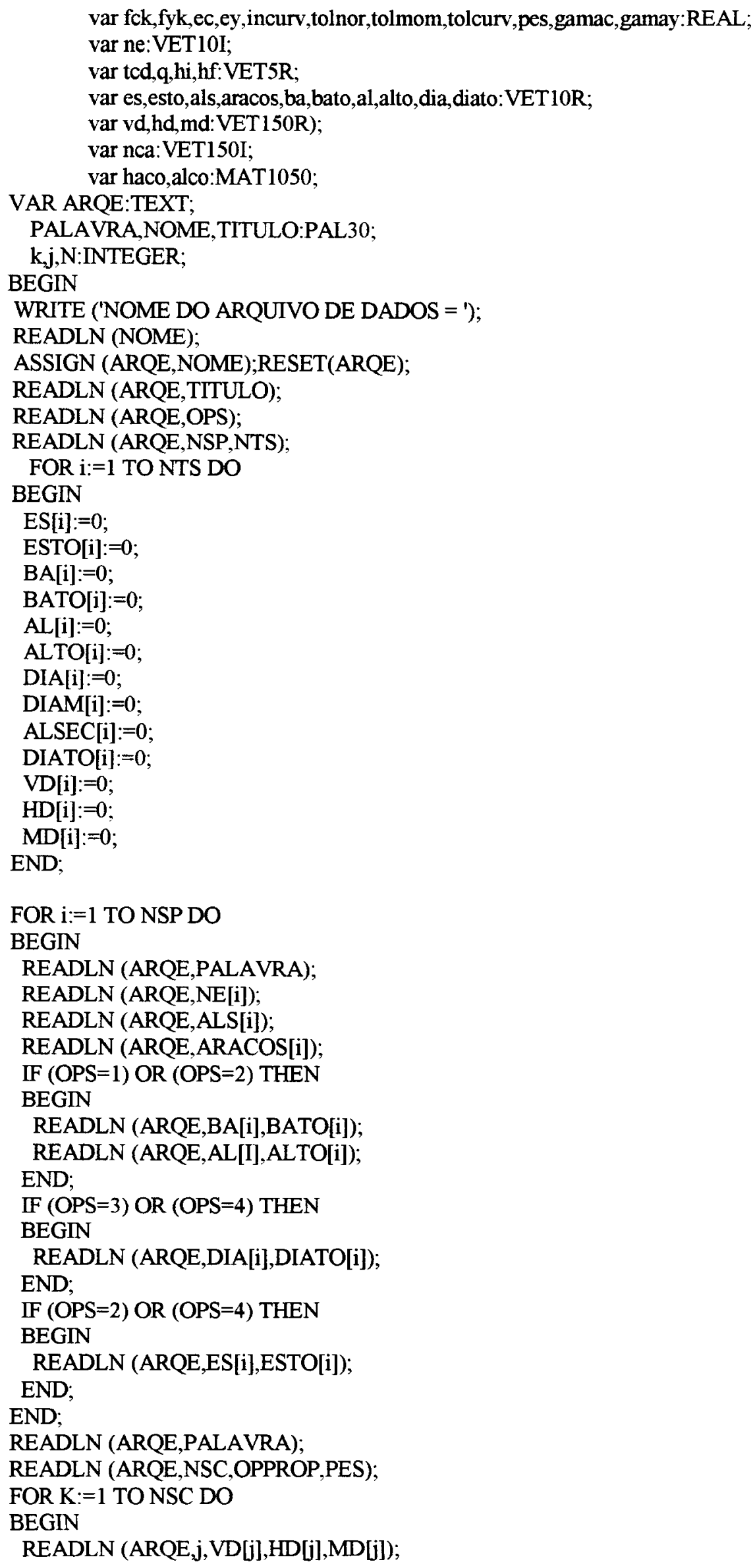




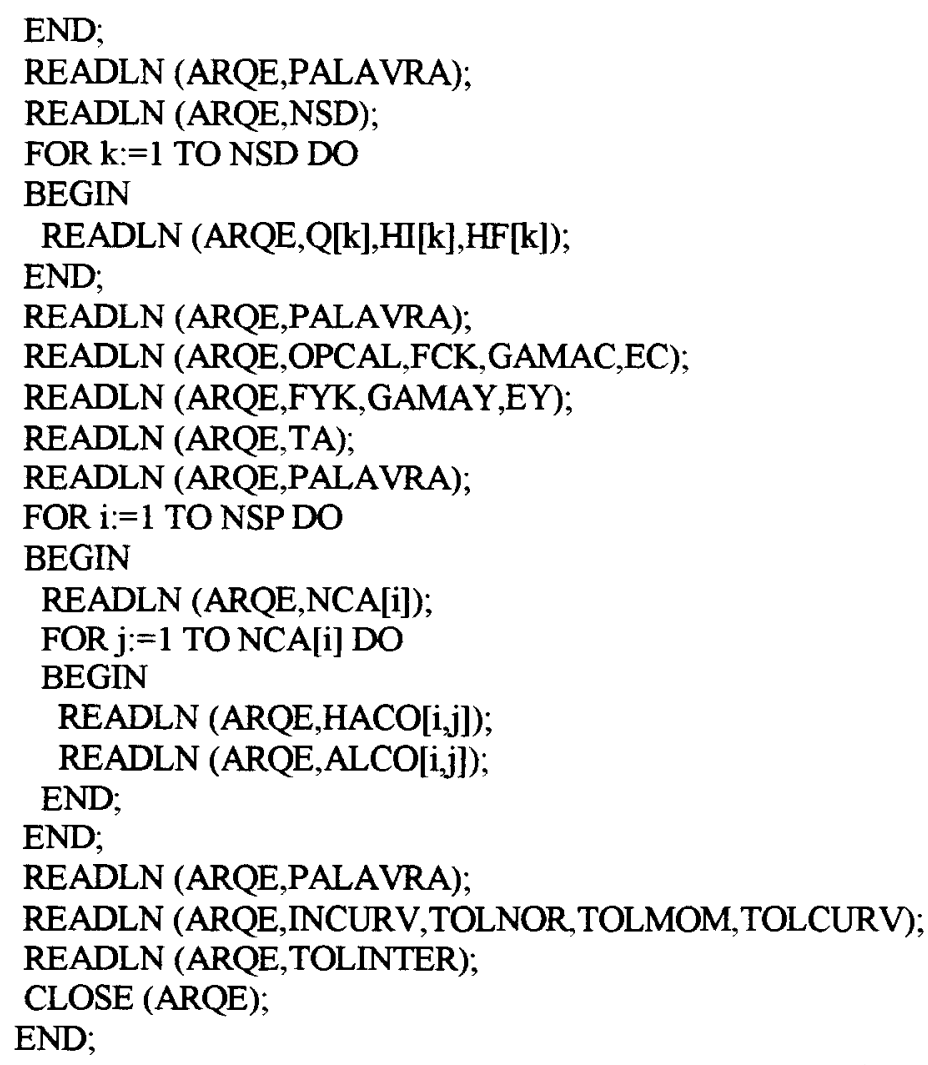

PROCEDURE DIMENSOES (var ops,nsp,nts,n:INTEGER; var fyk,fck, gamay,gamac:REAL; var als,es,esto,ba,bato,al,alto,aracos, dia, diato:VET10R; var base,altur,espes,ssec,taxarm, diam:VET150R; var ne:VET10I);

VAR difba,difal,difdia,difes:REAL; i,j:INTEGER;

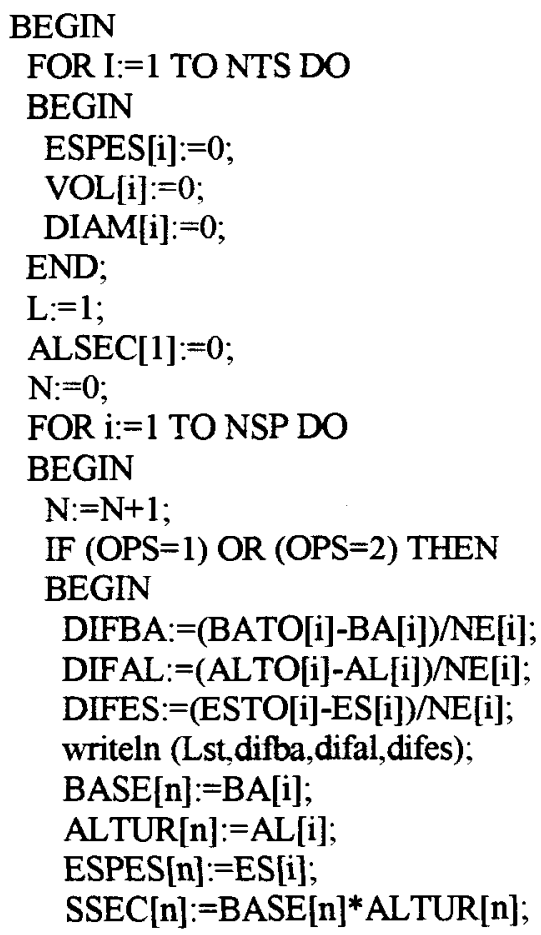




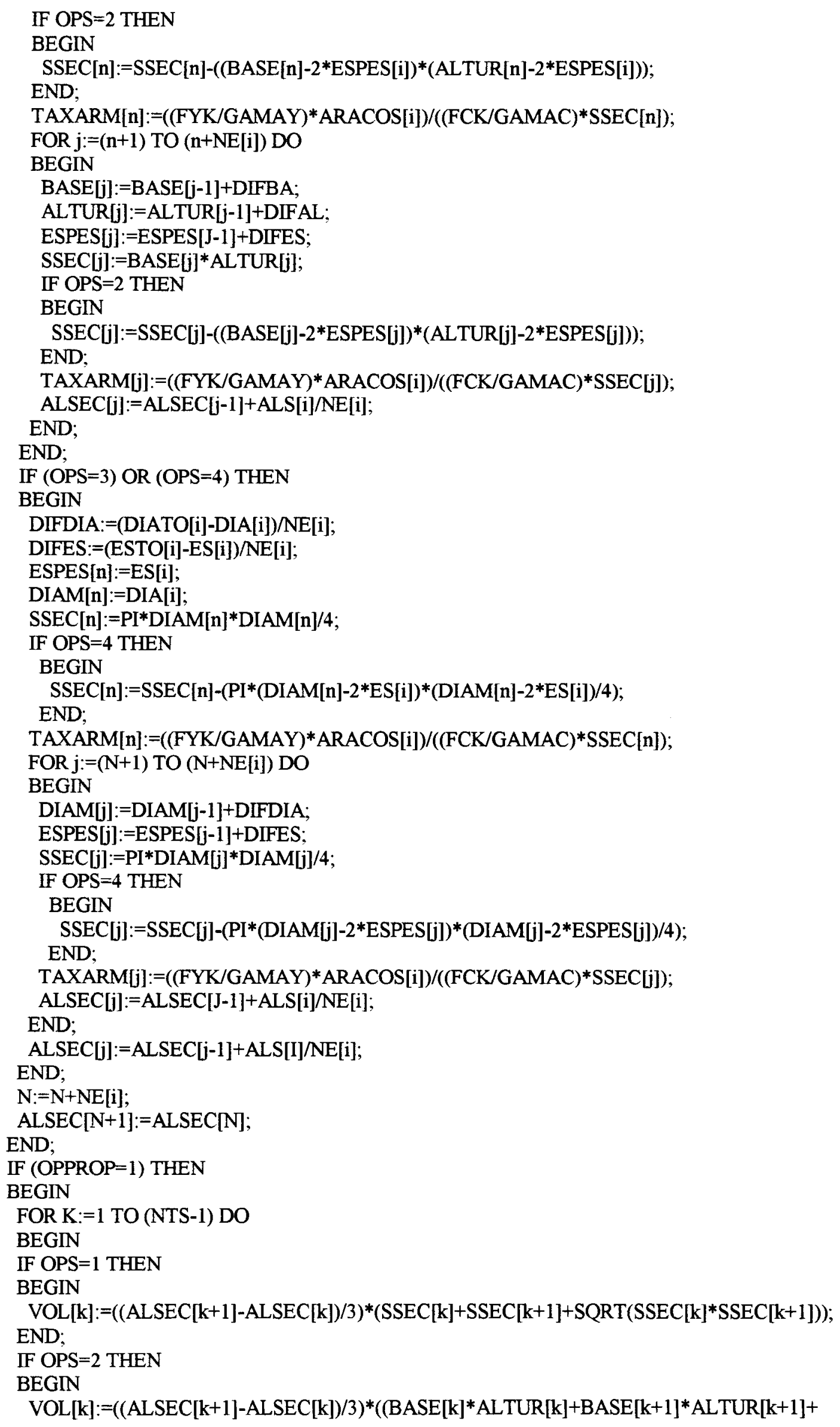




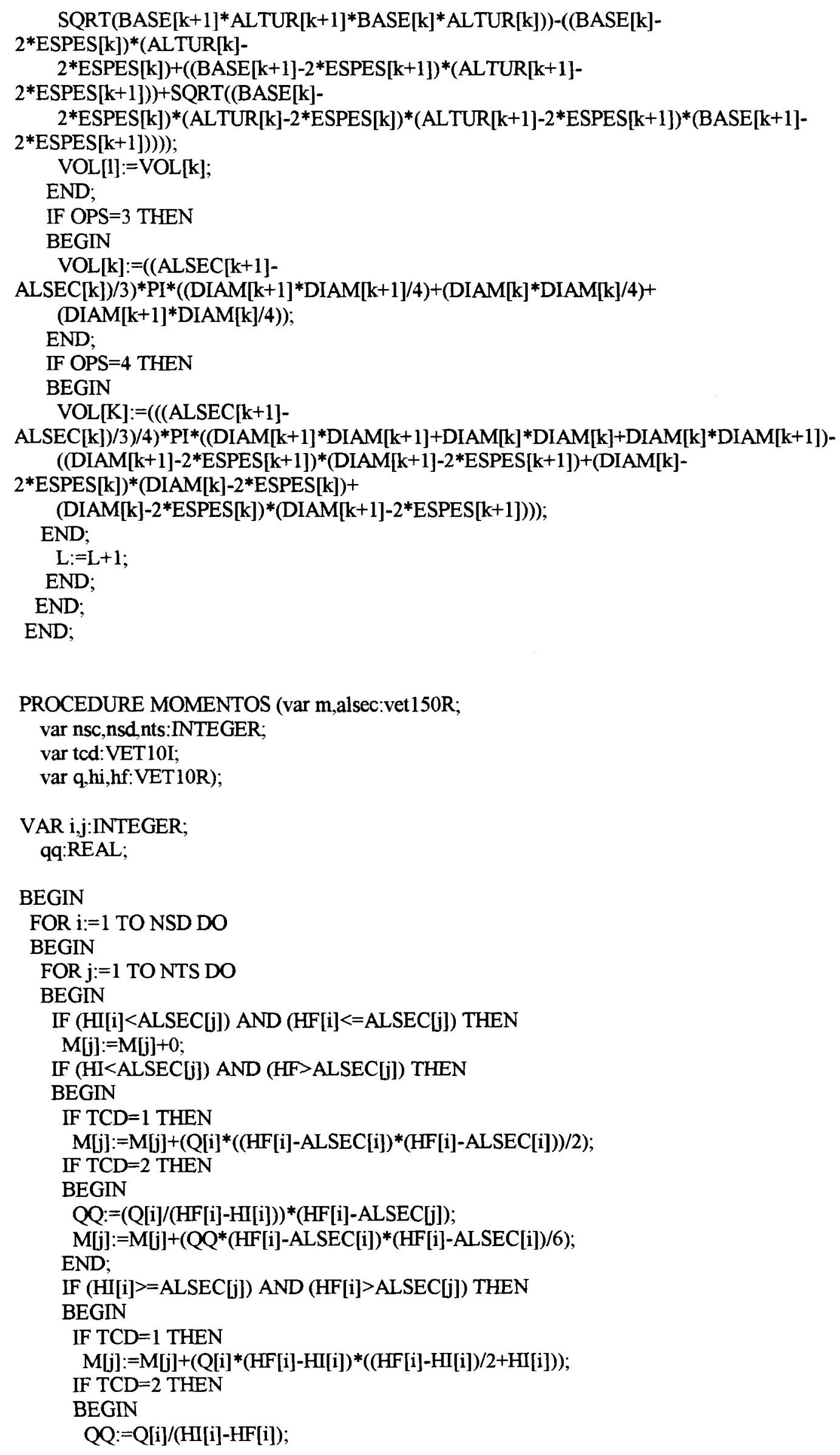




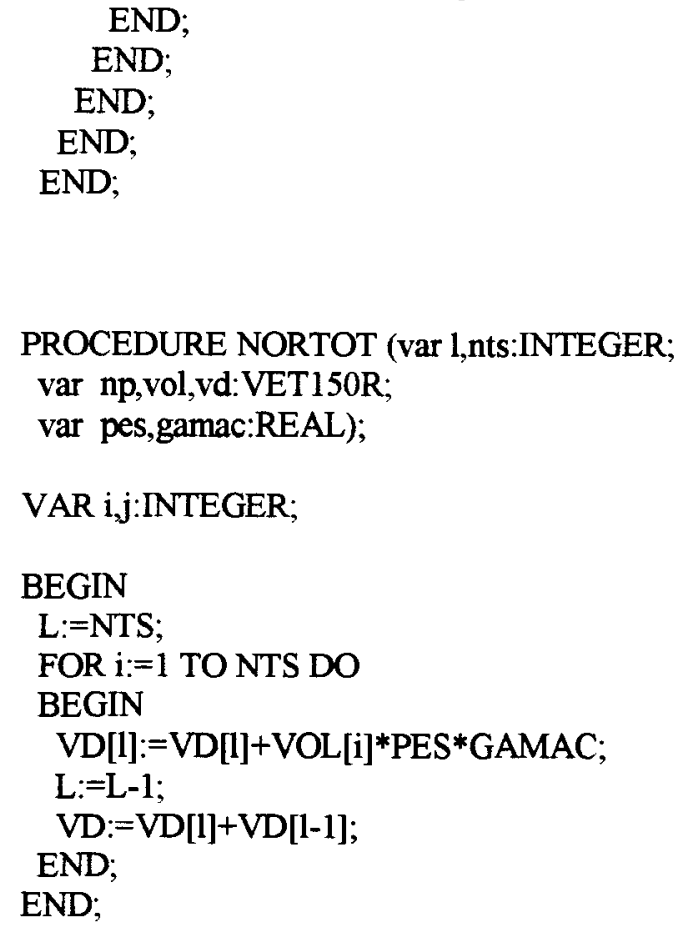

PROCEDURE ADIMEN (VAR va,vd,ssec,m,desloc,alsec,ma,diam:vet150R; var ops,nts:INTEGER;

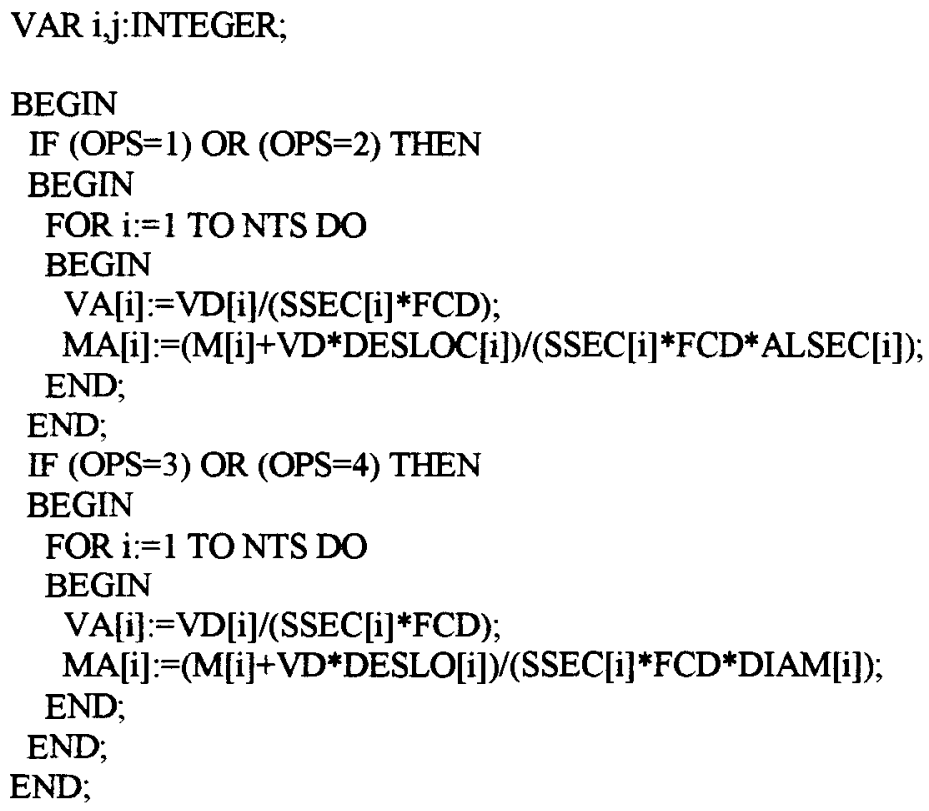




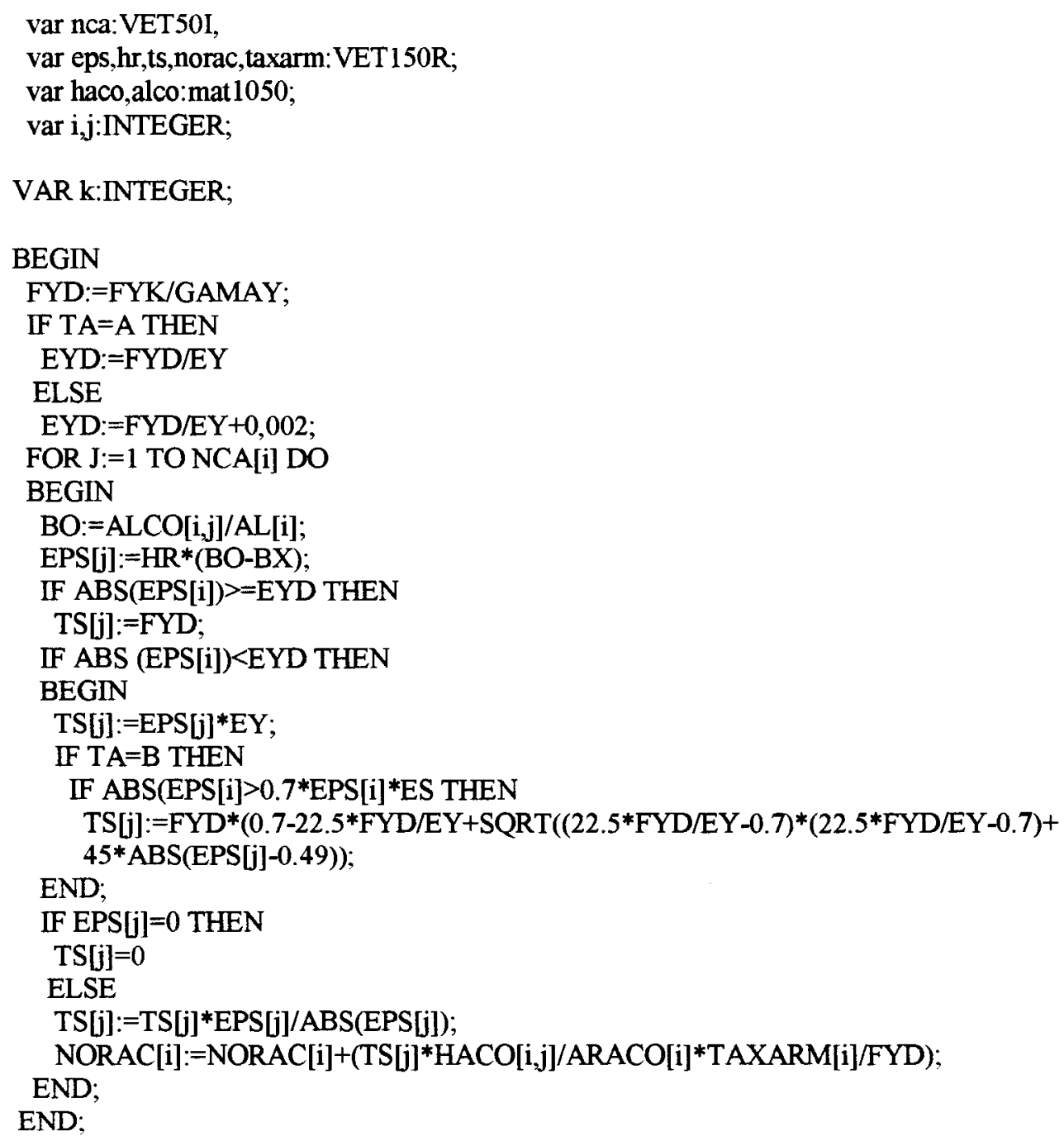

PROCEDURE MOMNOR (VAR bts,bti,bt1,btx,bt2,bt3,tol,na,tolnor, aux,aux1,aux2,btx1,btx2,norres1,norres2:REAL;

VAR k1,k2,k3,k4,k5,k6,k7,k8,k9,k10,k11,k12,k12,n1,n2,n3,n4,n5,n6,n7,n8, $\mathrm{n} 9, \mathrm{n10}, \mathrm{n} 11, \mathrm{al}, \mathrm{a} 2, \mathrm{a} 3, \mathrm{cl}, \mathrm{c2}, \mathrm{c3}, \mathrm{c4}, \mathrm{c5}, \mathrm{c6}, \mathrm{c} 7, \mathrm{c8}, \mathrm{c9}, \mathrm{cl} 0, \mathrm{c1} 1, \mathrm{c12}$ :REAL;

VAR contrl:INTEGER;

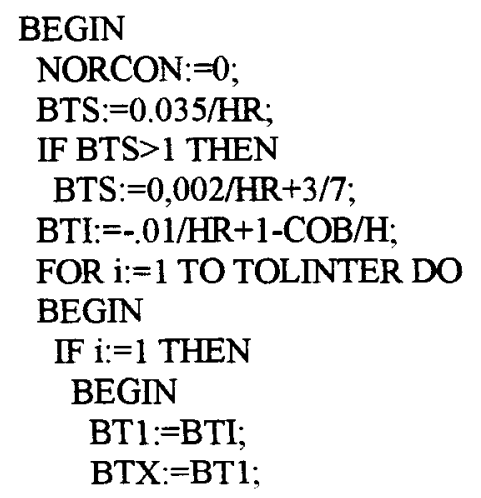




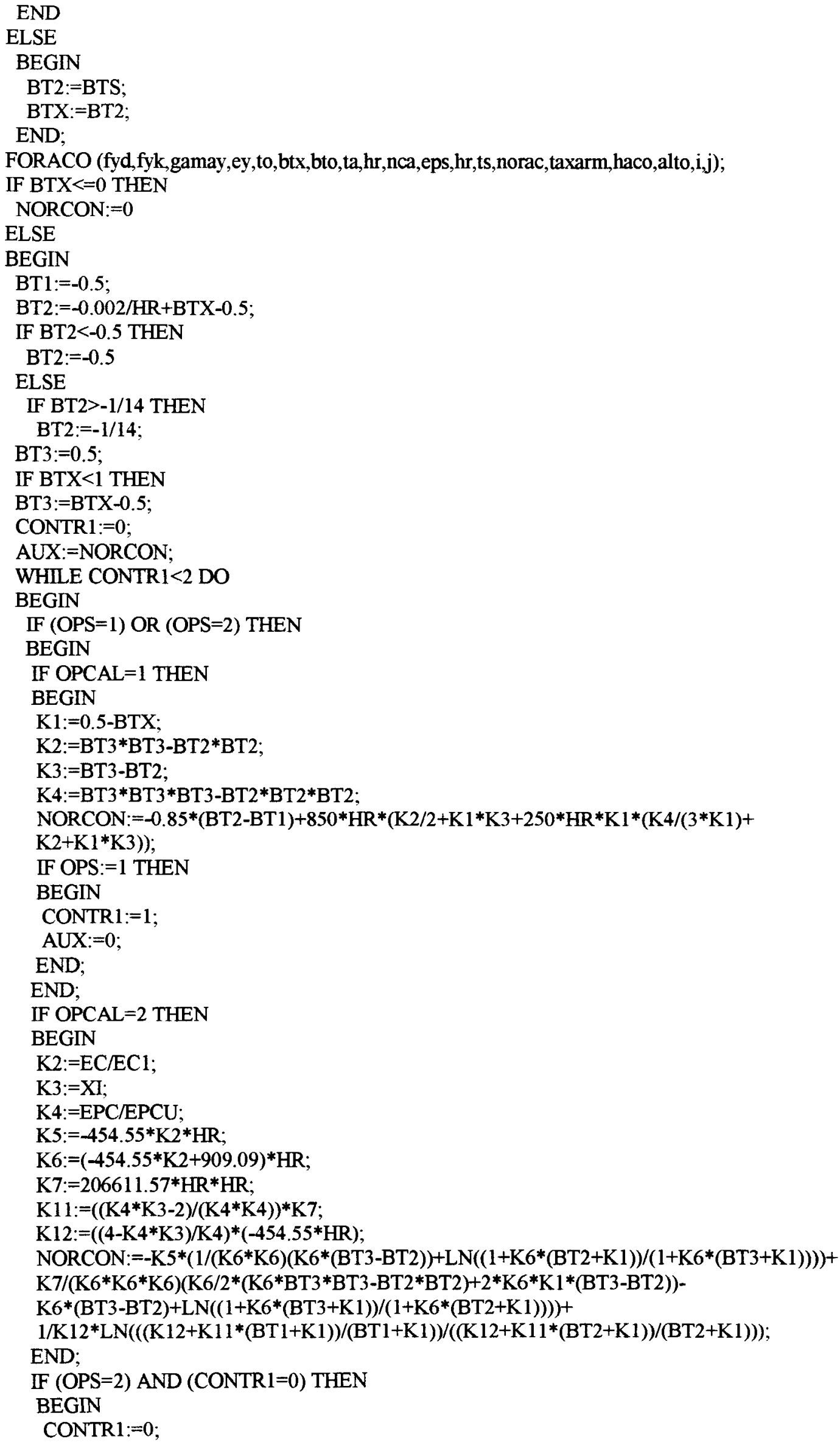




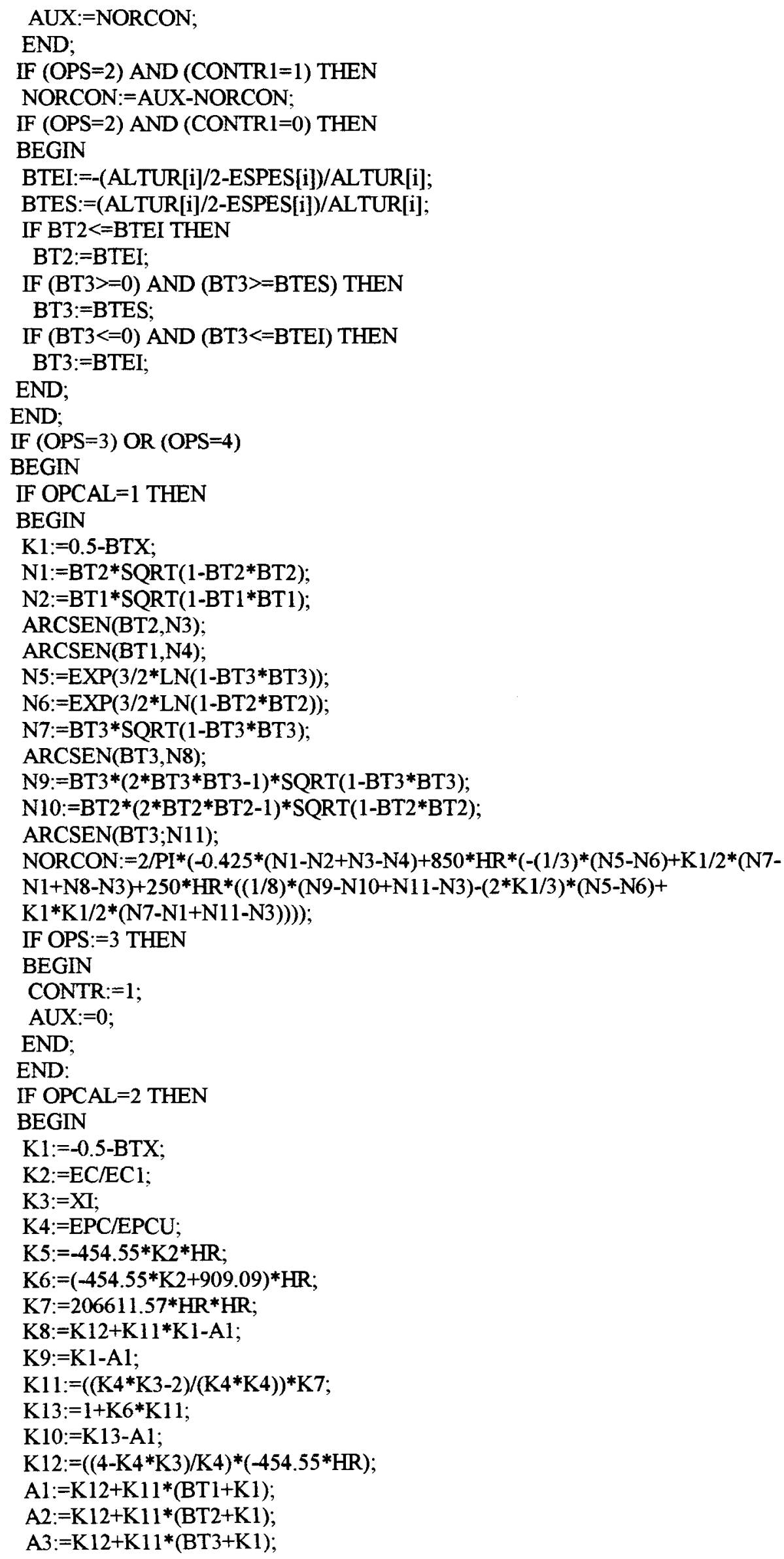


$\mathrm{N} 1:=\mathrm{SQRT}(1-\mathrm{BT} 2 * \mathrm{BT} 2)$;

$\mathrm{N} 2:=\mathrm{LN}(\mathrm{ABS}((\mathrm{K} 12+\mathrm{K} 11 *(\mathrm{BT} 2+\mathrm{K} 1)) /(\mathrm{BT} 2+\mathrm{K} 1)))$;

ARCSEN(BT2,N3);

ARCSEN(BT1,N4);

N5:=SQRT(1-BT3*BT3)

ARCSEN(BT3,N6);

N7:=SQRT(1-BT3*BT3);

ARCSEN(BT3,N8);

$\mathrm{Cl}:=\mathrm{LN}(\mathrm{A} 2)$;

$\mathrm{C} 2:=1 / \mathrm{A} 2$;

$\mathrm{C} 3:=1 /(2 * \mathrm{~A} 2 * \mathrm{~A} 2)$

$\mathrm{C} 4:=1 /(3 * \mathrm{~A} 2 * \mathrm{~A} 2 * \mathrm{~A} 2)$;

$\mathrm{C} 5:=\mathrm{LN}(\mathrm{A} 1)$;

$\mathrm{C} 6:=1 / \mathrm{Al}$;

$\mathrm{C} 7:=1 /(2 * \mathrm{~A} 1 * \mathrm{~A} 1)$

$\mathrm{C} 8:=1 /(3 * \mathrm{Al} * \mathrm{Al} * \mathrm{~A} 1)$;

$\mathrm{C} 9:=\mathrm{LN}(\mathrm{A} 3)$;

$\mathrm{C} 10:=1 / \mathrm{A} 3$;

$\mathrm{C} 11:=1 /(2 * \mathrm{~A} 3 * \mathrm{~A} 3)$;

$\mathrm{C} 12:=1 /\left(3^{*} \mathrm{~A} 3^{*} \mathrm{~A} 3 * \mathrm{~A} 3\right)$;

NORCON: $=2 / \mathrm{PI} *(\mathrm{~N} 1 / \mathrm{K} 12 * \mathrm{~N} 2+1 / \mathrm{K} 12 *(-\mathrm{C} 1 * \mathrm{~N} 1+\mathrm{C} 2 *((\mathrm{~K} 11 / 2) *(\mathrm{~N} 3-\mathrm{BT} 2 * \mathrm{~N} 1)-$

$\mathrm{K} 8 * \mathrm{~N} 1)-\mathrm{C} 3 *(-\mathrm{K} 11 * \mathrm{~K} 11 *((\mathrm{BT} 2 * \mathrm{BT} 2) * \mathrm{~N} 1 / 3)+\mathrm{K} 11 * \mathrm{~K} 8 *(\mathrm{~N} 3 * \mathrm{BT} 2-\mathrm{BT} 2 * \mathrm{~N} 1)-$

$\mathrm{K} 8 * \mathrm{~K} 8 * \mathrm{~N} 1)+\mathrm{C} 4 *(\mathrm{EXP}(3 * \mathrm{LN}(\mathrm{K} 11))) *(3 * \mathrm{~N} 3 / 8-\mathrm{BT} 2 * \mathrm{BT} 2 * \mathrm{BT} 2 * \mathrm{~N} 1 / 4-3 * \mathrm{BT} 2 * \mathrm{~N} 1 / 8)-$

$3 * \mathrm{~K} 11 * \mathrm{~K} 8 *(\mathrm{BT} 2 * \mathrm{BT} 2+2) * \mathrm{~N} 1 / 3+3 * \mathrm{~K} 11 * \mathrm{~K} 8 * \mathrm{~K} 8 / 2(\mathrm{~N} 3-\mathrm{BT} 2 * \mathrm{~N} 1)-\mathrm{EXP}(3 * \mathrm{LN}(\mathrm{N} 1))-$

$\left.\left(-\mathrm{C} 5 * \mathrm{~N} 1+\mathrm{C} 6{ }^{*} 0.5 * \mathrm{~N} 3-\mathrm{BT} 2 * \mathrm{~N} 1\right)-\mathrm{K} 9 * \mathrm{~N} 1\right)-\mathrm{C} 7 *(-(\mathrm{BT} 2 * \mathrm{BT} 2+2) * \mathrm{~N} 1 / 3+\mathrm{K} 9 * \mathrm{~N} 3-\mathrm{BT} 2 * \mathrm{~N} 1)-$

$\mathrm{K} 9 * \mathrm{~K} 9 * \mathrm{~N} 1+\mathrm{C} 8 *(3 * \mathrm{~N} 3 / 8-\mathrm{EXP}(3 * \mathrm{LN}(\mathrm{BT} 2)) * \mathrm{~N} 1 / 4-3 * \mathrm{BT} 2 * \mathrm{~N} 1 / 8-3 * \mathrm{~K} 9 *(\mathrm{BT} 2 * \mathrm{BTT} 2 * \mathrm{~N} 1 / 3)+$

$3 * \mathrm{~K} 9 * \mathrm{~K} 9 *(\mathrm{~N} 1 * \mathrm{BT} 2 * \mathrm{~N} 1)-\mathrm{EXP}(3 * \mathrm{LN}(\mathrm{K} 9)) * \mathrm{~N} 1)))-\mathrm{K} 5 *((\mathrm{~N} 5 /(\mathrm{K} 6 * \mathrm{~K} 6)) *(1+\mathrm{K} 6 *(\mathrm{BT} 3+\mathrm{K} 1)-$

$\mathrm{LN}(\mathrm{ABS}(1+\mathrm{K} 6 *(\mathrm{BT} 3+\mathrm{K} 1)))+\mathrm{l} /(\mathrm{K} 6 * \mathrm{~K} 6) *(-\mathrm{N} 5+\mathrm{K} 6 *(\mathrm{BT} 3 *(\mathrm{BT} 3+\mathrm{K} 1) * \mathrm{~N} 6-(\mathrm{BT} 3 * \mathrm{BT} 3) * \mathrm{~N} 6-$

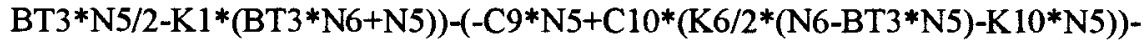

$\mathrm{C} 11 *(-\mathrm{K} 6 * \mathrm{~K} 6 *(\mathrm{BT} 2 * \mathrm{BT} 3) * \mathrm{~N} 5 / 3)+\mathrm{K} 6 * \mathrm{~K} 10 *(\mathrm{~N} 6-\mathrm{BT} 3 * \mathrm{~N} 5)-\mathrm{K} 10 * \mathrm{~K} 10)+$

$\mathrm{C} 12 *(\mathrm{EXP}(3 * \mathrm{LN}(\mathrm{K} 6)) * 3 * \mathrm{~N} 6 / 8-\mathrm{BT} 3 * \mathrm{BT} 3 * \mathrm{BT} 3 * \mathrm{~N} 5 / 4-3 * \mathrm{BT} 3 * \mathrm{~N} 5 / 8)-$

$3 * \mathrm{~K} 6 * \mathrm{~K} 6 * \mathrm{~K} 10 *((\mathrm{BT} 2 * \mathrm{BT} 3+2) * \mathrm{~N} 5 / 3)+3 * \mathrm{~K} 6 * \mathrm{~K} 10 * \mathrm{~K} 10 *(\mathrm{~N} 6-\mathrm{BT} 3 * \mathrm{~N} 5)-$

$(\operatorname{EXP}(3 * \mathrm{LN}(\mathrm{K} 10)) * \mathrm{~N} 5))+$

$\mathrm{K} 7 *(\mathrm{~N} 7 /(\mathrm{EXP}(3 * \mathrm{LN}(\mathrm{K} 6)) *(0.5 *(1+\mathrm{K} 6 *(\mathrm{BT} 2+\mathrm{K} 1) *(1+\mathrm{K} 6 *(\mathrm{BT} 2+\mathrm{K} 1))-$

$2 *(1+\mathrm{K} 6 *(\mathrm{BT} 2+\mathrm{K} 1))+$

$\mathrm{LN}(\mathrm{ABS}(1+\mathrm{K} 6 *(\mathrm{BT} 2+\mathrm{K} 1)))+1 /(\mathrm{EXP}(3 * \mathrm{LN}(\mathrm{K} 6))) *(0.5 *(-\mathrm{K} 6 * \mathrm{~K} 13 *(\mathrm{~N} 7-\mathrm{N} 8)-$

$\left.\mathrm{N} 7 *\left(\mathrm{~K} 13 * \mathrm{~K} 13+\mathrm{K} 6{ }^{*} \mathrm{~K} 6 *(\mathrm{BT} 2 * \mathrm{BT} 2+2) / 3\right)\right)-\mathrm{K} 6 * \mathrm{~N} 8+(\mathrm{K} 6 * \mathrm{BT} 2+2 * \mathrm{~K} 13) * \mathrm{~N} 7-\mathrm{C} 9 * \mathrm{~N} 7+$

$\mathrm{C} 10^{*}((\mathrm{~K} 6 / 2) *(\mathrm{~N} 8-\mathrm{N} 7)-\mathrm{K} 10 * \mathrm{~N} 7)-\mathrm{C} 11(-\mathrm{K} 6 *(\mathrm{BT} 2 * \mathrm{BT} 2+2) * \mathrm{~N} 7 / 3)+\mathrm{K} 6 . \mathrm{K} 10 *(\mathrm{~N} 8-$

$\mathrm{BT} 2 * \mathrm{~N} 7)-\mathrm{K} 10 * \mathrm{~K} 10 * \mathrm{~N} 7)+\mathrm{C} 12 *((\mathrm{EXP}(3 * \mathrm{LN}(\mathrm{K} 6)) * 3 * \mathrm{~N} 8 / 8-\mathrm{EXP}(3 * \mathrm{LN}(\mathrm{BT} 2)) / 4-$

$3 * \mathrm{BT} 2 * \mathrm{~N} 7 / 8)-3 * \mathrm{~K} 6 * \mathrm{~K} 6 * \mathrm{~K} 10 *(\mathrm{BT} 2 * \mathrm{BT} 2+2) * \mathrm{~N} 7 / 3+3 * \mathrm{~K} 6{ }^{*} \mathrm{~K} 10 * \mathrm{~K} 10 *(\mathrm{~N} 8-\mathrm{N} 7)-$

$\operatorname{EXP}(3 * \mathrm{LN}(\mathrm{N} 7)))))$ );

IF (OPS=4) AND (CONTR1=0) THEN

BEGIN

CONTR $1:=0$;

AUX:=NORCON;

END;

IF (OPS=4) AND (CONTR1=1) THEN

NORCON:=AUX-NORCON;

IF (OPS=4) AND (CONTR1=0) THEN

BEGIN

BTEI:=-(DIAM[i]/2-ESPES[i])/DIAM[i];

BTES:=(DIAM[i]/2-ESPES[i])/DIAM[i];

IF BT2<=BTEI THEN

$\mathrm{BT} 2:=\mathrm{BTEI} ;$

IF (BT3>=0) AND (BT3>=BTES) THEN

BT3:=BTES;

IF $($ BT3 $<=0)$ AND $($ BT3 $<=$ BTEI) THEN

BT3:=BTEI;

END; 


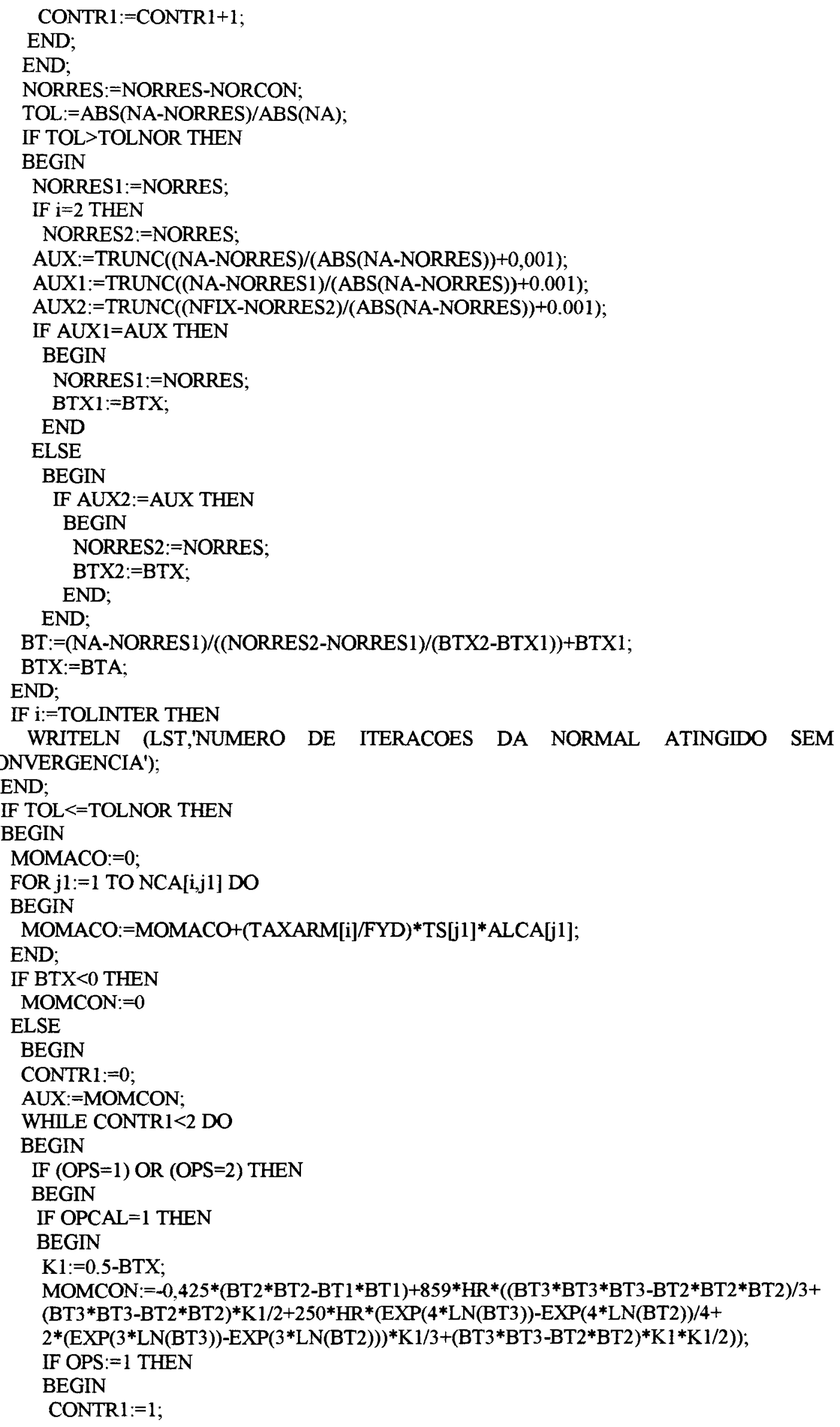




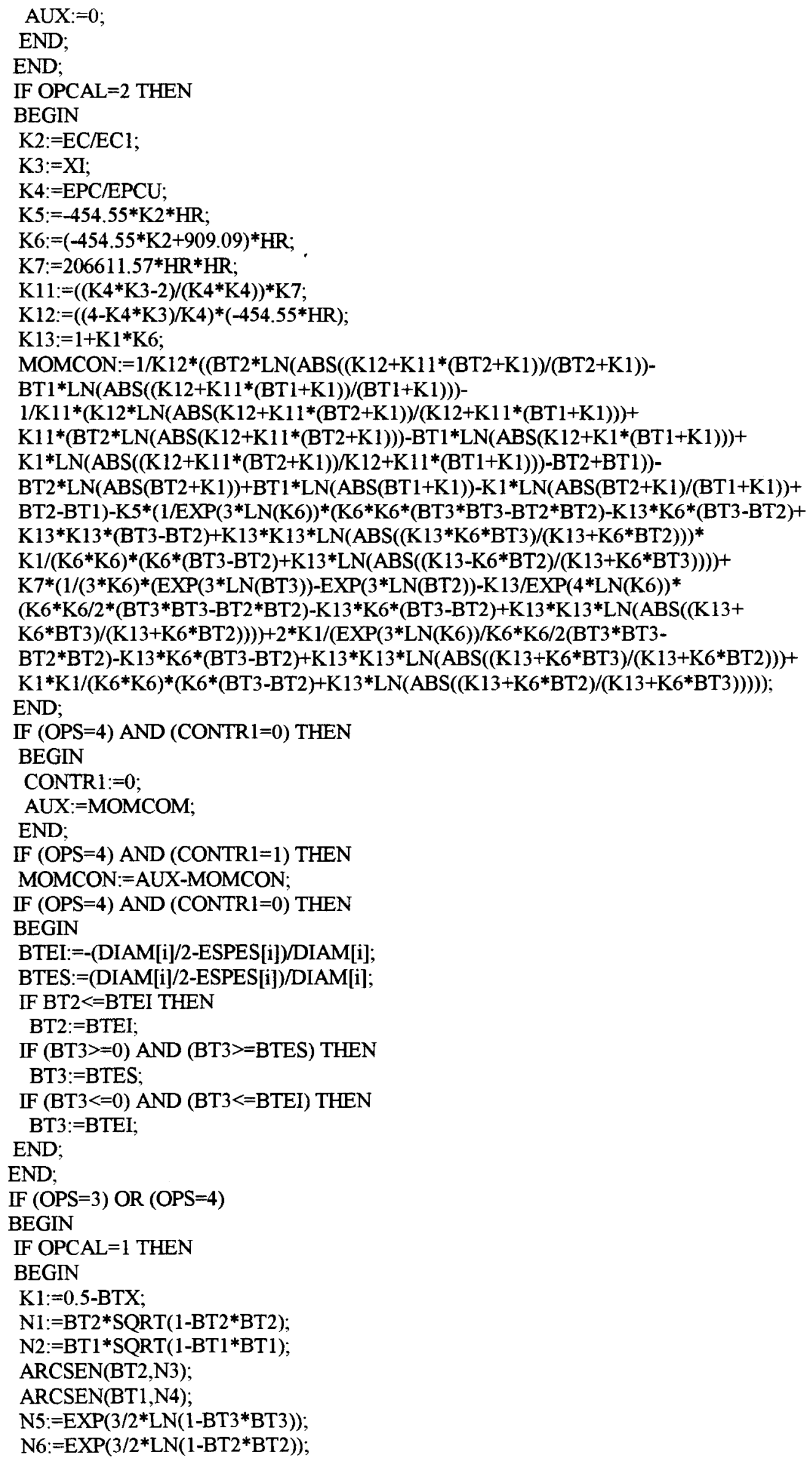


$\mathrm{N} 7:=\mathrm{BT} 3 * \mathrm{SQRT}(1-\mathrm{BT} 3 * \mathrm{BT} 3)$;

ARCSEN(BT3,N8);

N9:=BT3*(2*BT3*BT3-1)*SQRT(1-BT3*BT3);

$\mathrm{N} 10:=\mathrm{BT} 2 *(2 * \mathrm{BT} 2 * \mathrm{BT} 2-1) * \mathrm{SQRT}(1-\mathrm{BT} 2 * \mathrm{BT} 2)$;

ARCSEN(BT3;N11);

NORCON: $=2 / \mathrm{PI}^{*}(-0.425 *(\mathrm{~N} 1-\mathrm{N} 2+\mathrm{N} 3-\mathrm{N} 4)+850 * \mathrm{HR} *(-(1 / 3) *(\mathrm{~N} 5-\mathrm{N} 6)+\mathrm{K} 1 / 2 *(\mathrm{~N} 7-$

$\mathrm{N} 1+\mathrm{N} 8-\mathrm{N} 3)+250 * \mathrm{HR} *((1 / 8) *(\mathrm{~N} 9-\mathrm{N} 10+\mathrm{N} 11-\mathrm{N} 3)-(2 * \mathrm{~K} 1 / 3) *(\mathrm{~N} 5-\mathrm{N} 6)+$

$\mathrm{K} 1 * \mathrm{~K} 1 / 2 *(\mathrm{~N} 7-\mathrm{N} 1+\mathrm{N} 11-\mathrm{N} 3)))$ );

IF OPS: $=3$ THEN

BEGIN

CONTR: $=1$;

AUX: $=0$;

END;

END:

IF OPCAL $=2$ THEN

BEGIN

$\mathrm{K} 1:=-0.5$-BTX;

$\mathrm{K} 2:=\mathrm{EC} / \mathrm{EC} 1$;

$\mathrm{K} 3:=\mathrm{XI}$;

$\mathrm{K} 4:=\mathrm{EPC} / \mathrm{EPCU}$;

$\mathrm{K} 5:=-454.55 * \mathrm{~K} 2 * \mathrm{HR}$;

$\mathrm{K} 6:=(-454.55 * \mathrm{~K} 2+909.09) * \mathrm{HR}$;

$\mathrm{K} 7:=206611.57 * \mathrm{HR} * \mathrm{HR}$;

$\mathrm{K} 8:=\mathrm{K} 12+\mathrm{K} 11 * \mathrm{~K} 1-\mathrm{Al}$;

$\mathrm{K} 9:=\mathrm{K} 1-\mathrm{Al}$;

$\mathrm{K} 11:=((\mathrm{K} 4 * \mathrm{~K} 3-2) /(\mathrm{K} 4 * \mathrm{~K} 4)) * \mathrm{~K} 7$;

$\mathrm{K} 13:=1+\mathrm{K} 6 * \mathrm{~K} 11$;

$\mathrm{K} 10:=\mathrm{K} 13-\mathrm{A} 1$;

$\mathrm{K} 12:=((4-\mathrm{K} 4 * \mathrm{~K} 3) / \mathrm{K} 4) *(-454.55 * \mathrm{HR})$;

$\mathrm{Al}:=\mathrm{K} 12+\mathrm{K} 11 *(\mathrm{BT} 1+\mathrm{K} 1)$;

$\mathrm{A} 2:=\mathrm{K} 12+\mathrm{K} 11 *(\mathrm{BT} 2+\mathrm{K} 1)$;

$\mathrm{A} 3:=\mathrm{K} 12+\mathrm{K} 11 *(\mathrm{BT} 3+\mathrm{K} 1)$;

$\mathrm{N} 1:=\mathrm{SQRT}(1-\mathrm{BT} 2 * \mathrm{BT} 2)$;

$\mathrm{N} 2:=\mathrm{LN}(\mathrm{ABS}((\mathrm{K} 12+\mathrm{K} 11 *(\mathrm{BT} 2+\mathrm{K} 1)) /(\mathrm{BT} 2+\mathrm{K} 1)))$;

ARCSEN(BT2,N3);

ARCSEN(BT1,N4);

N5:=SQRT(1-BT3*BT3)

ARCSEN(BT3,N6);

$\mathrm{N} 7:=\mathrm{SQRT}(1-\mathrm{BT} 3 * \mathrm{BT} 3)$;

ARCSEN(BT3,N8);

$\mathrm{C} 1:=\mathrm{LN}(\mathrm{A} 2)$;

$\mathrm{C} 2:=1 / \mathrm{A} 2$;

$\mathrm{C} 3:=1 /(2 * \mathrm{~A} 2 * \mathrm{~A} 2)$

$\mathrm{C} 4:=1 /(3 * \mathrm{~A} 2 * \mathrm{~A} 2 * \mathrm{~A} 2)$;

$\mathrm{C} 5:=\mathrm{LN}(\mathrm{Al})$;

$\mathrm{C} 6:=1 / \mathrm{Al}$

$\mathrm{C} 7:=1 /(2 * \mathrm{Al} * \mathrm{Al})$;

$\mathrm{C} 8:=1 /\left(3^{*} \mathrm{Al}{ }^{*} \mathrm{Al}{ }^{*} \mathrm{Al}\right)$;

C $9:=\mathrm{LN}(\mathrm{A} 3)$;

$\mathrm{Cl} 1=1 / \mathrm{A} 3$;

$\mathrm{C} 11:=1 /(2 * \mathrm{~A} 3 * \mathrm{~A} 3)$

$\mathrm{C} 12:=1 /(3 * \mathrm{~A} 3 * \mathrm{~A} 3 * \mathrm{~A} 3)$

MOMCON: $=2 / \mathrm{PI} *\left((-\mathrm{K} 1 / \mathrm{K} 12+1 / \mathrm{K} 11) * \mathrm{LN}^{*}(\mathrm{ABS}(\mathrm{K} 11 *(\mathrm{BT} 2+\mathrm{K} 1) * \mathrm{~K} 12))-\right.$

$\mathrm{K} 1 * \mathrm{LN}(\mathrm{ABS}(\mathrm{BT} 2+\mathrm{K} 1)) / \mathrm{K} 12) * \mathrm{~N} 1+(\mathrm{K} 1 / \mathrm{K} 12+1 / \mathrm{K} 11) *(-\mathrm{C} 1 * \mathrm{~N} 1+\mathrm{C} 2 *(\mathrm{~K} 11 / 2 *(\mathrm{~N} 2-$

$\left.\mathrm{BT} 2 * \mathrm{~N} 1)-\mathrm{K} 8 * \mathrm{~N} 1))-\mathrm{C} 3 *\left(\mathrm{~K} 11 * \mathrm{~K} 11 *\left((\mathrm{BT} 2 * \mathrm{BT} 2)^{*} \mathrm{~N} 1 / 3\right)\right)+\mathrm{K} 11 * \mathrm{~K} 8 *(\mathrm{~N} 2 * \mathrm{BT} 2-\mathrm{BT} 2 * \mathrm{~N} 1)\right)-$ $\mathrm{K} 8 * \mathrm{~K} 8 * \mathrm{~N} 1)+\mathrm{C} 4 *\left(\mathrm{EXP}\left(3{ }^{*} \mathrm{LN}(\mathrm{K} 11)\right)\right) *(3 * \mathrm{~N} 3 / 8-\mathrm{BT} 2 * \mathrm{BT} 2 * \mathrm{BT} 2 * \mathrm{~N} 1 / 4-3 * \mathrm{BT} 2 * \mathrm{~N} 1 / 8)-$ $3 * \mathrm{~K} 11 * \mathrm{~K} 8 *(\mathrm{BT} 2 * \mathrm{BT} 2+2) * \mathrm{~N} 1 / 3+3 * \mathrm{~K} 11 * \mathrm{~K} 8 * \mathrm{~K} 8 / 2(\mathrm{~N} 3-\mathrm{BT} 2 * \mathrm{~N} 1)-\mathrm{EXP}(3 * \mathrm{LN}(\mathrm{N} 1))-$ $\left.\left(-\mathrm{C} 5 * \mathrm{~N} 1+\mathrm{C}^{*} 0.5 * \mathrm{~N} 3-\mathrm{BT} 2 * \mathrm{~N} 1\right)-\mathrm{K} 9 * \mathrm{~N} 1\right)-\mathrm{C} 7 *(-(\mathrm{BT} 2 * \mathrm{BT} 2+2) * \mathrm{~N} 1 / 3+\mathrm{K} 9 * \mathrm{~N} 3-\mathrm{BT} 2 * \mathrm{~N} 1)-$ $\mathrm{K} 9 * \mathrm{~K} 9 * \mathrm{~N} 1+\mathrm{C} 8 *\left(3 * \mathrm{~N} 3 / 8-\mathrm{EXP}\left(3{ }^{*} \mathrm{LN}(\mathrm{BT} 2)\right) * \mathrm{~N} 1 / 4-3 * \mathrm{BT} 2 * \mathrm{~N} 1 / 8-3 * \mathrm{~K} 9 *(\mathrm{BT} 2 * \mathrm{BT} 2 * \mathrm{~N} 1 / 3)+\right.$ 


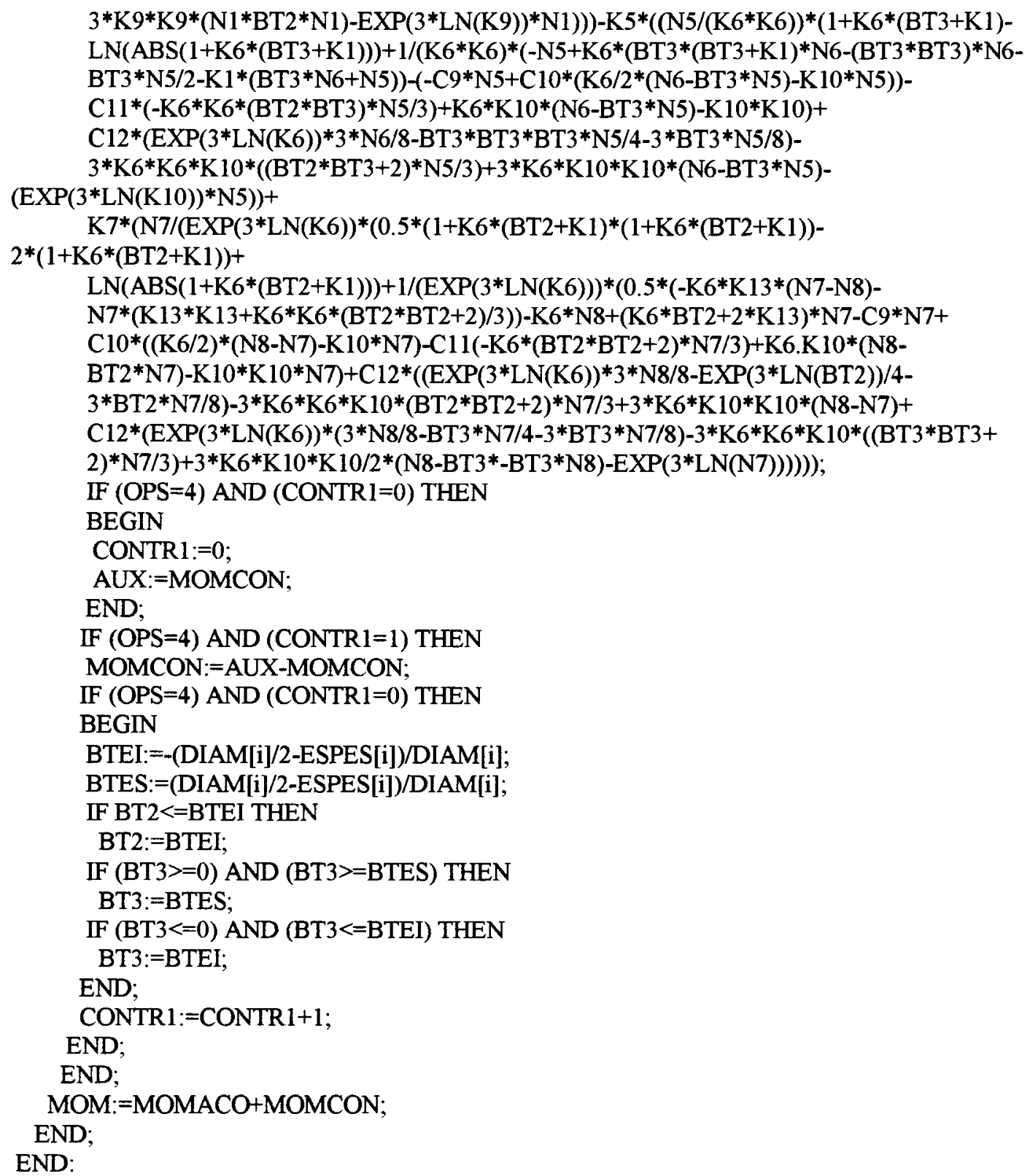

PROCEDURE DESLOC (VAR nts:integer; var hr,rot,desloc:vet150R;

VAR j,k,ki,kj:integer; aux1,aux2:real;

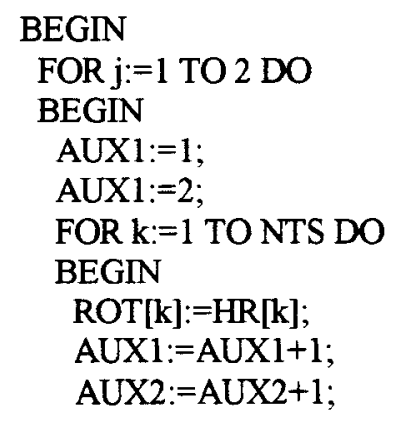




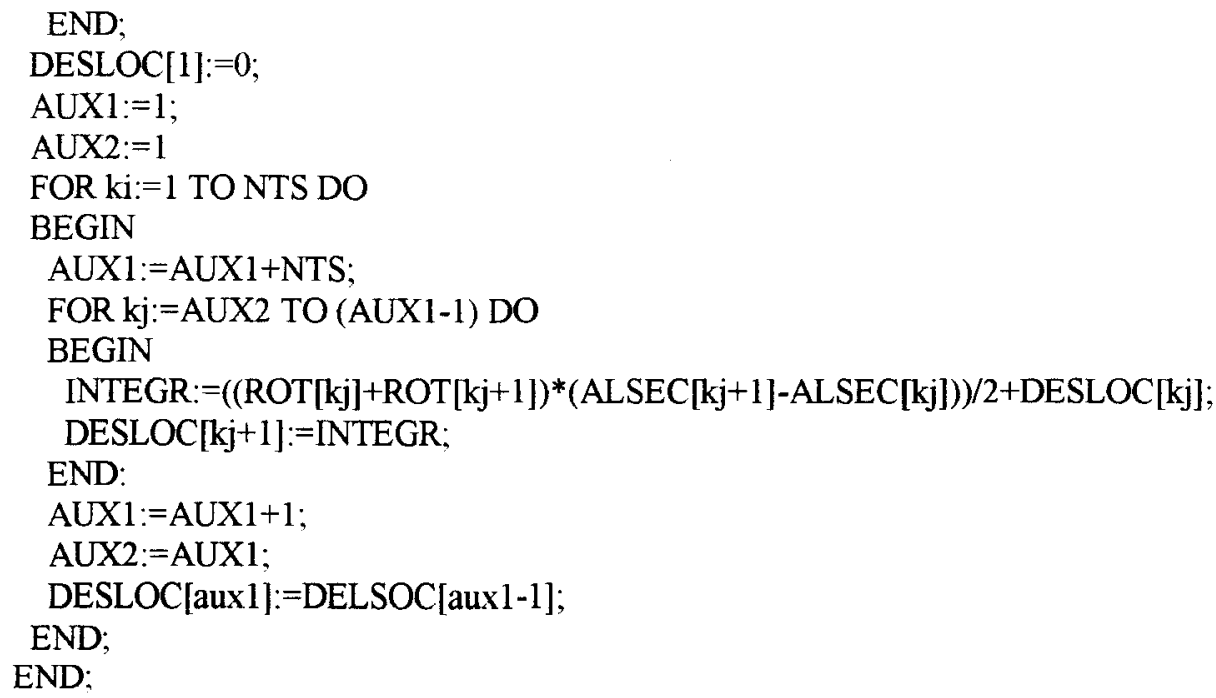

DIMENSOES (ops,nsp,nts,n,fyk,fck,gamay,gamac.als,es, esto, ba,bato,al,alto,aracos. dia.diato,base, altur,espes, ssec.taxarm, diam.ne):

MOMENTOS (m,alsec,nsc,nsd,nts,tcd,m.q,hi,hf);

IF OPPROP=1 THEN

NORTOT (1,nts,np,vol,vd,pes,gamac);

for $i:=1$ to nts do

begin

writeln (Lst,base[i],altur[i], espes[i]);

writeln (Lst.ssec[i],taxarm[i],alsec[i]);

writeln (Lst,diam[i]);

writeln (Lst,vol[i]);

end:

ADIMEN (va.vd,ssec,m,desloc.alsec,ma,diam,ops,nts.fcd);

FYD $:=F Y K / G A M A Y:$

$\mathrm{FCD}:=\mathrm{FCK} / \mathrm{GAMAC}$; 


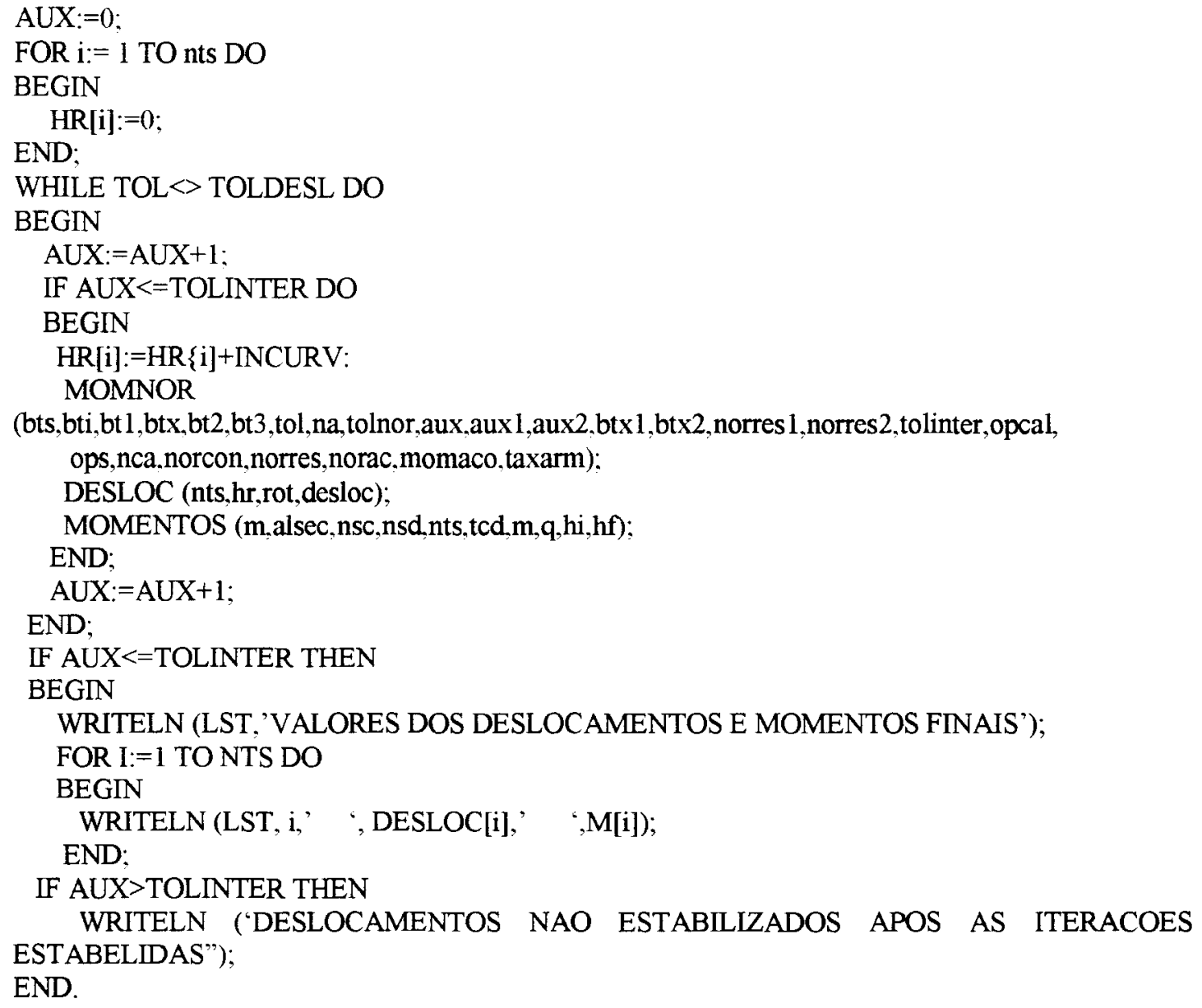




\section{CONCLUSÃO}

Além da revisão de conceitos de resistência dos materiais, da descrição do comportamento do concreto e do aço sob tensões segundo a NBR-6118/1978 e o CEB-1990 e da recomendações destas normas, relativas à verificação da estabilidade de pilares isolados, este trabalho apresenta o desenvolvimento das expressões adimensionais (normais e momentos) utilizadas na determinação da curvatura das seções de pilares de concreto armado submetidos à flexão normal composta.

As expressões adimensionais acima citadas foram incorporadas ao programa computacional aqui apresentado. As recomendações do CEB levam à obtenção de deslocamentos menores do que aqueles obtidos com as recomendações da NBR, decorrente da adoção de um valor menor do coeficiente de minoração da tensão de resistência à compressão do concreto.

O programa aqui apresentado pode ser utilizado em estudos comparativos dos parâmetros de cálculo adotados, podendo-se incorporar ao mesmo rotinas que se destinem à avaliação dos efeitos da fluência, adaptação de outros tipos de vinculação ou outras alterações que o tornem mais abrangente.

$\mathrm{O}$ volume de cálculo implicado na verificação da estabilidade de pilares pelo método geral, principalmente quando são seguidas as diretrizes do CEB, requer a utilização de rotinas computacionais. O processo de Engesser-Vianello, que requer ábacos para as relações momento fletor - força normal - curvatura, torna-se um recurso de verificação aproximada, devido às interpolações que se fazem necessárias ao crescente acesso aos equipamentos computacionais. 


\section{ANEXO I \\ CÁLCULO DO MOMENTO DE INÉRCIA DE FLAMBAGEM}

$\mathrm{Na}$ fig. A.1 estão representados os casos de vinculação $\mathrm{e}$ as formas das barras abrangidas pela tabela A.l, que é utilizada para a determinação do momento de inércia fictício de barras troncônicas ou tronco prismáticas, com uma extremidade livre e outra engastada.

Além dos casos I e II, representados nas figuras A. $1^{\text {a }}$ e Al.b, a tabela A.1 abrange o caso de uma barra biengastada (caso III), cuja variação da seção transversal seja a mesma da fig. A.1b. Para o caso III, tem-se como comprimento de flambagem $l_{\mathrm{fl}}=l$, de acordo com a notação utilizada na figura A.1.

$\mathrm{O}$ valor a ser atribuído à variável a, utilizada na determinação da razão $\mathrm{a} / l$ da primeira coluna da tabela A. $1 \mathrm{~b}$. Os valores de $\mathrm{n}$ (constante dependente da forma e da seção da barra) são determinados de acordo com a fig. A.1c, onde as formas das barras estão apresentadas em elevação e em corte (AA).

Nos casos de barras troncônicas ou tronco prismáticas, os valores adotados para a e n são

$$
a=0 \quad \text { e } \quad n=4 .
$$

As notações usadas na fig. A.1 e na tabela A.1 são as que se seguem: 


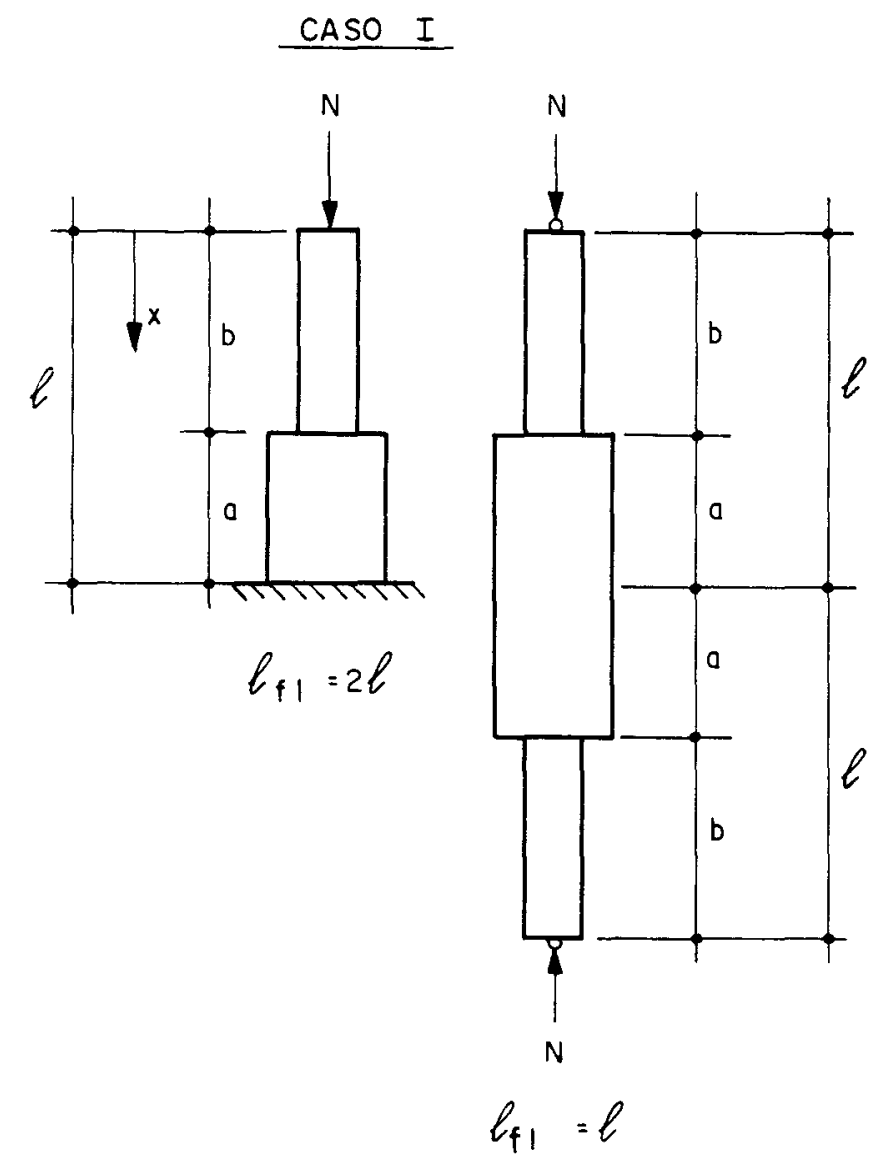

(a)
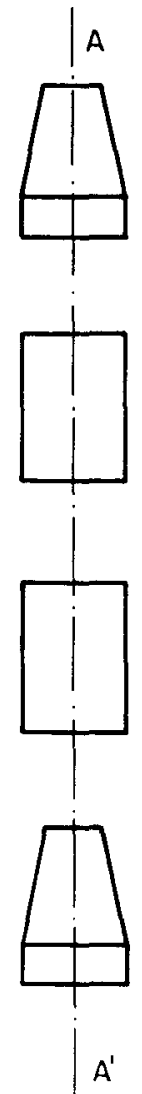

ELEVAÇÃO
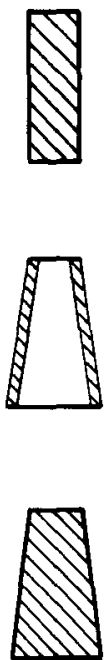

NIII
CASO II

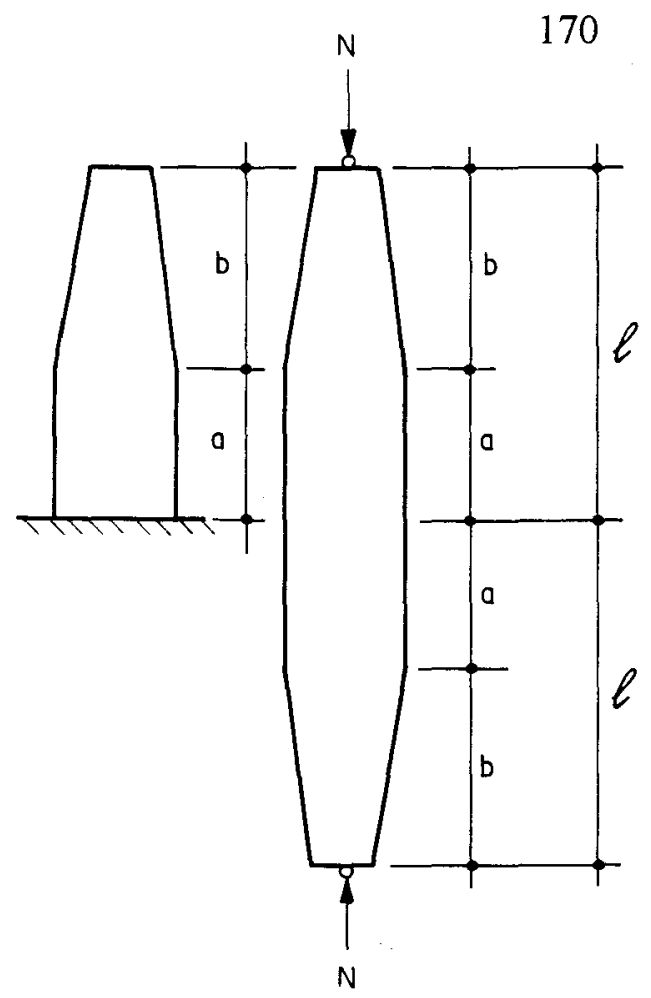

(b)

$n=1$

$n=2$

$n=3$

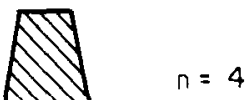

CORTE AA

(c)

Fig. A. 1 - Forma das barras abrangidas pela tabela A.1. 
$\mathrm{I}_{\alpha}, \mathrm{I}_{\mathrm{b}}, \mathrm{I}=$ momentos de inércia da seção transversal (em relação ao eixo que passa no centro de gravidade, perpendicularmente à direção de flambagem), respectivamente para $\mathrm{x}=0,0<\mathrm{x}<$ e $\mathrm{x} \geq$

$\mathrm{i}=\mathrm{I} \alpha / \mathrm{Ib}$

$\mathrm{n}=$ constante (ver fig. A .1c)

$\mathrm{a} / l=$ razão entre o valor a correspondente a cada barra e o comprimento 1 da barra (ver figuras A . la e A .1b).

As tabelas A.1 e A. 2 fornecem o valor da razão entre o momento de inércia fictício de flambagem e o momento de inércia Ib (já definido anteriormente).

No caso de pilares com carga intermediária, ver na figura A.2 os casos abrangidos, a tabela utilizada é a A.2, onde as notações são as mesmas já citadas e $\mathrm{N}$ é a carga axial aplicada na barra. Para a utilização desta tabela, determina-se a razão N2/N1 (ver fig. A.2).
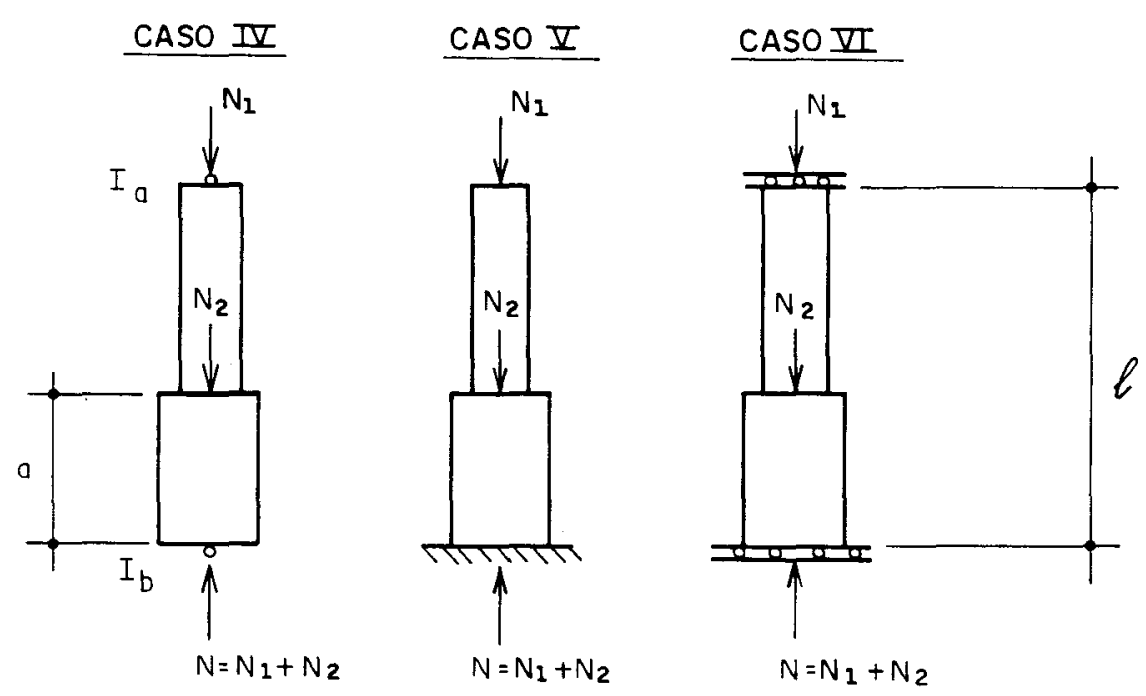

Fig. A .2 - Casos de vinculação correspondentes à tabela A.2. 
Tabela A . 1 - Valores de $\mathrm{I}_{\mathrm{fl}} / \mathrm{I}_{\mathrm{b}}$.

\begin{tabular}{|c|c|c|c|c|c|c|c|c|c|c|}
\hline \multirow{2}{*}{$\frac{\mathrm{a}}{\mathrm{l}}$} & \multirow{2}{*}{$i$} & \multirow{2}{*}{ caso I } & \multicolumn{4}{|c|}{ caso II } & \multicolumn{4}{|c|}{ caso III } \\
\hline & & & $\mathrm{n}=1$ & $n=2$ & $n=3$ & $\mathrm{n}=4$ & $n=1$ & $\mathrm{n}=2$ & $n=3$ & $n=4$ \\
\hline \multirow[t]{6}{*}{0} & 0,01 & - & 0,59 & 0,35 & 0,26 & 0,22 & - & - & - & - \\
\hline & 0,1 & - & 0,66 & 0,55 & 0,51 & 0,49 & - & - & - & - \\
\hline & 0,2 & - & 0,71 & 0,65 & 0,62 & 0,61 & 0,52 & 0,48 & 0,47 & 0,42 \\
\hline & 0,4 & - & 0,80 & 0,77 & 0,76 & 0,76 & 0,66 & 0,65 & 0,64 & 0,64 \\
\hline & 0,6 & - & 0,87 & 0,86 & 0,86 & 0,86 & 0,79 & 0,78 & 0,78 & 0,78 \\
\hline & 0,8 & - & 0,94 & 0,94 & 0,94 & 0,94 & 0,90 & 0,90 & 0,89 & 0,89 \\
\hline \multirow{6}{*}{0,2} & 001 & 0015 & 072 & 048 & 037 & 032 & _ & _ & _ & _ \\
\hline & 0,1 & 0,15 & 0,77 & 0,68 & 0,64 & 0,62 & - & _ & _ & _ \\
\hline & 0,2 & 0,28 & 0,81 & 0,76 & 0,74 & 0,73 & 0,57 & 0,54 & 0,53 & 0,52 \\
\hline & 0,4 & 0,52 & 0,87 & 0,85 & 0,85 & 0,84 & 0,70 & 0,69 & 0,69 & 0,69 \\
\hline & 0,6 & 0,71 & 0,92 & 0,92 & 0,91 & 0,91 & 0,82 & 0,81 & 0,81 & 0,81 \\
\hline & 0,8 & 0,87 & 0,97 & 0,96 & 0,96 & 0,96 & 0,91 & 0,91 & 0,91 & 0,91 \\
\hline \multirow[t]{6}{*}{0,4} & 0,01 & 0,027 & 0,85 & 0,67 & 0,55 & 0,49 & - & - & - & - \\
\hline & 0,1 & 0,24 & 0,87 & 0,82 & 0,79 & 0,78 & - & - & - & - \\
\hline & 0,2 & 0,43 & 0,90 & 0,87 & 0,86 & 0,85 & 0,59 & 0,58 & 0,57 & 0,57 \\
\hline & 0,4 & 0,68 & 0,93 & 0,93 & 0,92 & 0,92 & 0,73 & 0,72 & 0,72 & 0,72 \\
\hline & 0,6 & 0,83 & 0,97 & 0,96 & 0,96 & 0,96 & 0,83 & 0,83 & 0,83 & 0,83 \\
\hline & 0,8 & 0,93 & 0,98 & 0,98 & 0,98 & 0,98 & 0,92 & 0,92 & 0,92 & 0,92 \\
\hline \multirow[t]{6}{*}{0,6} & 0,01 & 0,06 & 0,95 & 0,87 & 0,81 & 0,76 & - & - & - & - \\
\hline & 0,1 & 0,46 & 0,96 & 0,94 & 0,93 & 0,92 & - & - & - & - \\
\hline & 0,2 & 0,68 & 0,98 & 0,96 & 0,95 & 0,95 & 0,64 & 0,62 & 0,61 & 0,60 \\
\hline & 0,4 & 0,86 & 0,98 & 0,98 & 0,97 & 0,97 & 0,76 & 0,75 & 0,75 & 0,75 \\
\hline & 0,6 & 0,94 & 0,99 & 0,99 & 0,99 & 0,99 & 0,86 & 0,85 & 0,85 & 0,85 \\
\hline & 0,8 & 0,98 & 1,00 & 0,99 & 0,99 & 0,99 & 0,93 & 0,93 & 0,93 & 0,93 \\
\hline \multirow[t]{6}{*}{0,8} & 0,01 & 0,23 & 0,99 & 0,98 & 0,98 & 0,97 & - & - & - & - \\
\hline & 0,1 & 0,87 & 0,99 & 0,99 & 0,99 & 0,99 & - & - & - & - \\
\hline & 0,2 & 0,95 & 0,99 & 0,99 & 0,99 & 0,99 & 0,73 & 0,70 & 0,69 & 0,68 \\
\hline & 0,4 & 0,98 & 1,00 & 1,00 & 1,00 & 1,00 & 0,84 & 0,83 & 0,82 & 0,82 \\
\hline & 0,6 & 0,99 & 1,00 & 1,00 & 1,00 & 1,00 & 0,91 & 0,90 & 0,90 & 0,90 \\
\hline & 0,8 & 1,00 & 1,00 & 1,00 & 1,00 & 1,00 & 0,96 & 0,96 & 0,96 & 0,96 \\
\hline 1,0 & - & 1,00 & 1,00 & 1,00 & 1,00 & 1,00 & 1,00 & 1,00 & 1,00 & 1,00 \\
\hline
\end{tabular}

FONTE:- LANGENDONCK, 1959 - Cálculo de Concreto. 
Tabela A.2 - Valores de $\mathrm{I}_{\mathrm{f}} / \mathrm{I}_{6}$.

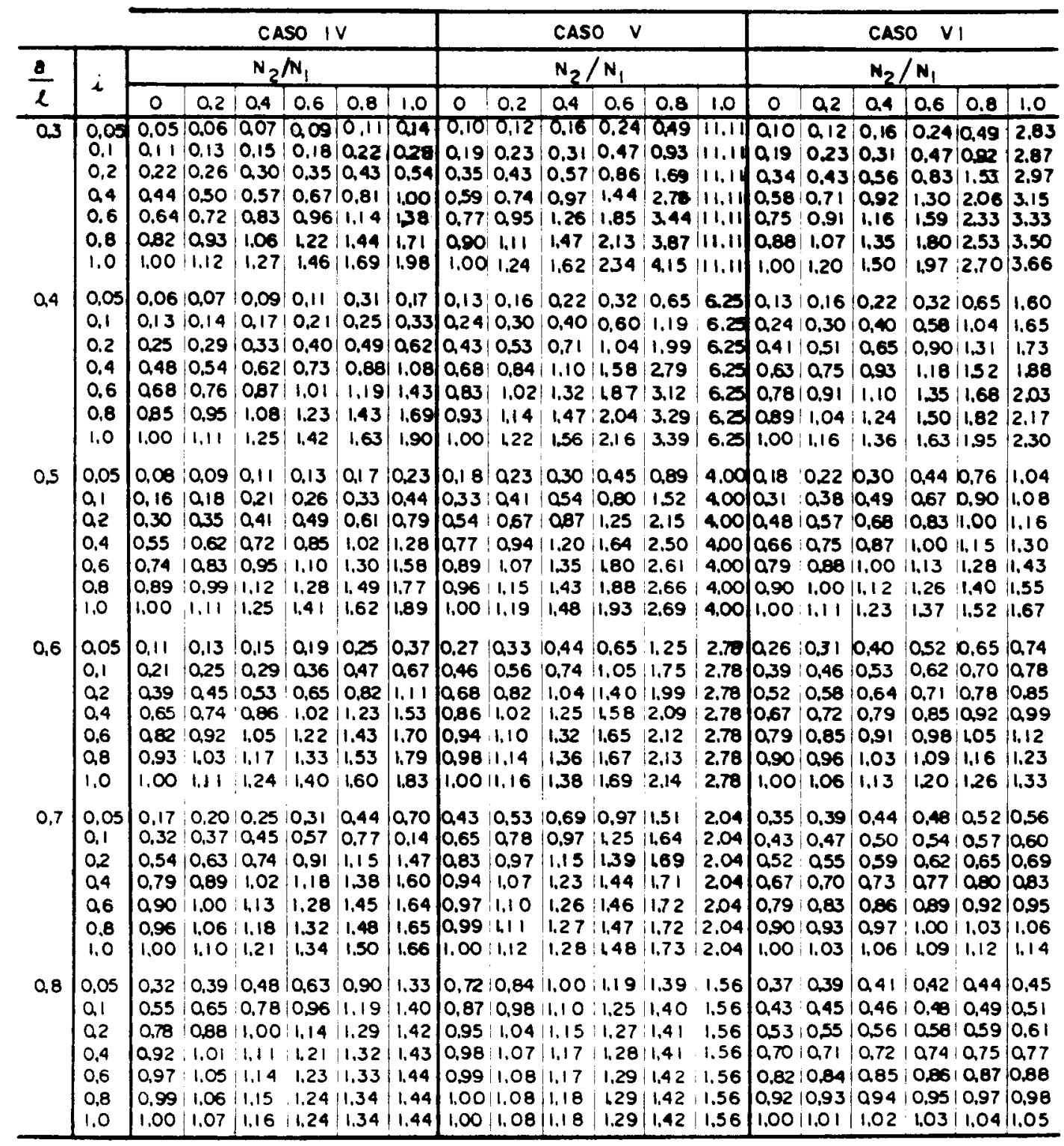

FONTE:- LANGENDONCK, 1959 - Cálculo de Concreto. 


\section{REFERÊNCIAS BIBLIOGRÁFICAS}

ASSOCIAÇÃO BRASILEIRA DE NORMAS TÉCNICAS. Projeto e execução de obras de concreto armado. Rio de Janeiro, 1978. (NBR-6118).

ASSOCIAÇÃO BRASILEIRA DE NORMAS TÉCNICAS. Projeto de estruturas de concreto protendido. Rio de Janeiro, 1989. (NBR-7197).

ASSOCIAÇÃO BRASILEIRA DE NORMAS TÉCNICAS. Ações e segurança nas estruturas. Rio de Janeiro, 1984. (NBR-8681).

CEB/FIB 1978. Manual of buckling and instability. Paris, V. 123 - Comité EuroInternacional du Béton.

CEB-FIB MODEL CODE 1990. Bulletins d'information. Paris, V. 203, 204 e 205 Comité Euro-Internacional du Béton, 1991.

DERIVE VERSION 2.01. A mathematical assistant. Honolulu - Hawai. Soft Warehouse, $1988-1990$.

EUROCODE-2. Design of Concrete Structures. Part 1: General rules and rules for buildings, 1989 .

FRANÇA, R.L.S. Contribuição ao estudo dos efeitos de segunda ordem em pilares de concreto armado. Tese de doutorado. Escola Politécnica da Universidade de São Paul, 1991.

FUSCO, P.B. Estruturas de concreto: solicitações normais. Rio de Janeiro. Guanabara Dois, 1986. 
GILBERT, R.I. Time effects in concrete structures. New York. Elsevier, 1988.

HOFFMANN, J. Pilares esbeltos de concreto armado: método exato. CT-18, UFRGS Porto Alegre - RS. 1980.

HUMES, A.F.P.C. Noções de cálculo numérico. São Paulo. Editora McGraw Hill, 1984. p. $158-179$.

LANGENDONCK, T.V. Cálculo do concreto Armado. $3^{\underline{a}}$ ed. Rio de Janeiro, Científica, 1959. V.01.

LEONHARDT, F. Construções de concreto. $2^{\mathrm{a}}$ ed. Rio de Janeiro, Interciência, 1977. P.247-296, V. 01. (Traduzido do original alemão Voriesungen über Massivbau - Erster Teil Grundlagen zur Bemessung in Stahlbetonbau).

LEONHARDT, F. Construções de concreto. $2^{\mathrm{a}}$ ed. Rio de Janeiro, Interciência, 1982. P.89-94 V. 06. (Traduzido do original alemão Voriesungen über Massivbau - Sechster Teil Grundlagen des Massivbrückenbaues).

MILNE, W.E. Cálculo numérico. São Paulo, Editora Polígono, 1968. p. 93-96.

PAULA, J.A. Algoritmos para o estudo de pilares esbeltos de concreto armado solicitados a flexão normal composta. Dissertação de mestrado. Escola de Engenharia de São Carlos EESC-USP, 1988.

PINHEIRO, M.P. [et al]. Concreto armado: ábacos para flexão oblíqua. Escola de Engenharia de São Carlos. 1994.

SUSSEKIND, J.C. Curso de análise estrutural. $2^{\text {a.- }}$ ed. Rio de Janeiro, Globo, 1987. V.03 